2011

\title{
Nanostructured organic solar cells
}

Dillip Kumar Panda

University of Wollongong

\section{Recommended Citation}

Panda, Dillip Kumar, Nanostructured organic solar cells, Doctor of Philosophy thesis, Department of Chemistry, University of Wollongong, 2011. http://ro.uow.edu.au/theses/3415 


\section{UNIVERSITY OF WOLLONGONG COPYRIGHT WARNING}

You may print or download ONE copy of this document for the purpose of your own research or study. The University does not authorise you to copy, communicate or otherwise make available electronically to any other person any copyright material contained on this site. You are reminded of the following:

Copyright owners are entitled to take legal action against persons who infringe their copyright. A reproduction of material that is protected by copyright may be a copyright infringement. A court may impose penalties and award damages in relation to offences and infringements relating to copyright material. Higher penalties may apply, and higher damages may be awarded, for offences and infringements involving the conversion of material into digital or electronic form. 


\section{NANOSTRUCTURED ORGANIC SOLAR CELLS}

A thesis submitted in fulfilment of the requirements for the degree

DOCTOR OF PHILOSOPHY

From

UNIVERSITY OF WOLLONGONG

by

DILLIP KUMAR PANDA

(B.Sc 1st Class Chem. Hons), M. Sc, 1st Class (University-Gold Medal)

Chemistry

DEPARTMENT OF CHEMISTRY

October 2011 



\section{DECLARATION}

I do hereby solemnly declare that the research carried out for my $\mathrm{PhD}$ thesis entitled "Nanostructured organic solar cells" in Intelligent Polymer Research Institute (I.P.R.I.), Department of Chemistry; University of Wollongong NSW, Australia is my own work (unless otherwise mentioned ) and this thesis has not been submitted for any qualifications at any other organisation, university or institute.

Name of student:

Signature:

Date: 


\section{ACKNOWLEDGEMENTS}

On this auspicious occasion I give profound thanks to all concerned who have extended their selfless guidance to assist in completing my $\mathrm{PhD}$ thesis. First, my most sincere regard and deep devotion goes to my supervisors Prof. Gordon Wallace and Dr. Attila Mozer. Prof. Wallace is a globally reputed scientific acrobat who, by the most sincere care, gave me rays of hope and aspiration to accomplish this thesis. I was very fortunate to work with my co-supervisor Dr. Attila Mozer, a global leader in solar cell research, who has taught and guided me in every aspect of my $\mathrm{PhD}$ thesis work since the day I started. He has helped me a lot without the slightest hesitation and I will never forget his scientific help during my PhD.

Secondly, I want to extend my thanks to Assoc. Prof. Chee Too and the Late Prof. Leon Kane-Maguire who helped with writing this thesis. I appreciated their time. Prof. David Officer and the Late Prof. Leon Kane-Maguire offered both technical support and scientific discussions during my $\mathrm{PhD}$, which were gratefully received. I ask for the blessings of the Lord to my parents, parents-in-law, school teachers (especially Rabindra sir, a high-school teacher), brothers, sisters, brothers-in-law, sisters-in-law, nephews, nieces, other family members, relatives, and the wishes of my beloved wife who has inspired me a lot to finish this task. On this day of thesis submission, I solemnly remember their help.

I would like to thank senior research fellows Michael Higgins, Tony Romeo, Lynn Dennany, David Harman and Bjorn Winther-Jensen (Monash University, Melbourne) for their technical help and fruitful discussion on AFM, SEM, Raman MALDI and vapour-phase polymerization work respectively.

I want to give sincere thanks to my lab mates George Tsekouras, Klaudia Wagner, Grant Mathieson, Matt Griffith, Jared Barnes and other lab members especially Sanjeev Gambhir, Satyen Desai, Pawel Wagner, Adrian Gestos, Damia Mawad, Jolly Locke, Dennis Antiohos, Syed Ashraf, Johanna Mbere, Paul Molino, and Scott McGovern. I also acknowledge the administrative help and fun from Phil (Fil4it) Bec 
and Karla during my Ph.D. At the end I am very much thankful and acknowledge to Prof. Gordon Wallace for providing me with an ACES-IPRI scholarship and the HDR scholarship from the University of Wollongong. Last but not least thanks go to all my dear friends Sanjay and Sujatha, Rao and Jyoti, Anita, Lei Tong, Yu Uemura, Subrat, Kumar, Naveen, Sreenu, Nandu, Prasanjit, Sujit, Sajith, and Khemchand who helped me directly and indirectly during my journey towards completion of my Ph.D. At the end, the love, blessing, affection, inspiration, motivation and patience shown by my parents was a great factor for success. This thesis is dedicated to my parents (Baba and Maa). 


\section{PUBLICATIONS}

1. Mozer, A. J., Panda, D. K., Gambhir, S., Winther-Jensen, B., Wallace, G.G., Journal of the American Chemical Society, 132 (2010) 9543-9545, "Microsecond dye regeneration kinetics inefficient solid state dye-sensitized solar cells using PEDOT a photoelectrochemiccally PEDOT as hole conductor".

2. Mozer, A. J., Panda, D. K., Gambhir, S., Romeo, T., Winther-Jensen, B., Wallace G.G, Langmuir, 26 (2010) 1452-1455, "Flexible and compressible Goretex-PEDOT membrane electrode for solid-state dye-sensitized solar cells".

3. Panda, D. K., Mozer, A. J., Wagner, P., Winther-Jensen, B., Officer, D.L., Wallace G.G., "Zinc-Porphyrin (GD2) dye as a photo-sensitiser for PEDOT based solid state dye-sensitized solar cells)" (in preparation).

4. Panda, D. K., Mozer, A. J., Gambhir, S., Officer, D.L., Wallace G.G., "Graphene as a solid-state dye-sensitised solar cell counter electrode" (in preparation).

5. Mozer, A. J., Panda, D. K., Gambhir, S., Romeo, T, C., Higgins, M, WintherJensen, B, Wallace, G. G., "Morphological determination of photo-anode grown by a photo-electrochemical method" (in preparation). 


\section{CONFERENCE PRESENTATIONS}

1. Panda, D. K., Mozer, A. J., Tsekouras, G., Wagner, P., Officer, D.L., Wallace G.G., "Conducting polymer based organic/Inorganic Solar Cells" (poster presentation), Full Centre Workshop, Melbourne, Australia, 3-4 October, 2007.

2. Panda, D. K., Mozer, A. J., Tsekouras, G., Wagner, P., Officer, D.L., Wallace, G.G., "Inorganic/organic hybrid solar cells using photo-electrochemically deposited electroactive polymers as hole transporting materials" (poster presentation), 17th International Photochemical Conversion and Storage of Solar Energy (IPS 17th) Sydney, Australia, 28 July-1 Aug 2008.

3. Panda, D. K., Mozer, A. J., Wallace G.G., "Solid State Dye sensitized solar cells (poster presentation", A European School in Materials Science" University of Milano-Bicocca, Milan, Italy, 14-19 September, 2009.

4. Panda, D. K., Mozer, A. J., Wallace G.G., "PEDOT based solid state solar cells" (poster presentation) Australian Centre of Electromaterials Science (ACES) Forum: Challenges in solar cells characterization: Theory, spectroscopy and devices, Innovation campus, University of Wollongong, Australia, 16 -17 Nov 2009. 


\section{AWARDS AND SCHOLARSHIPS}

1. Awarded an ARCNN (The Australian Research Council Nanotechnology Network) Scholarship to attend and present a poster on solar cells at the international conference 17th International Photochemical Conversion and Storage of Solar Energy (IPS 17th) at Sydney, July 2008.

2. Awarded an ARNAM (Australian Research Network for Advanced Materials) Scholarship to attend the IUMRS-ICEM international conference on electronic materials, Sydney, 28 July - 1 Aug 2008.

3. Awarded European Doctorate in Physics and Chemistry of Advanced Materials (PCAM) Fellowship to attend and present a poster at the "Chemistry and Physics of Materials for Energetics. A European School in Materials Science" in Milano, Italy from 14 -19 September 2009. Held at the University of Milano-Bicocca, (focus on new materials for renewable energy sources).

4. Awarded an ATSE (Australian Academy of Technological Sciences and Engineering) Early Career Symposium Fellowship to attend the 32nd ATSE National Symposium, Sofitel Brisbane Grand Central on 16-17 November 2009. The topic of this symposium was Future-Proofing Australia-Rising to the Challenge of Climate Change.

5. Awarded Equity Scholarship to attend the Third Annual Students 4 Students National Leadership Conference (S4S NLC) held at UOW, Wollongong, over 26-27 September 2009. 
ABSTRACT

A wide range of state-of-the art solid-state dye-sensitized solar cells (ssDSCs) have been fabricated employing in situ photo-electrochemical polymerization (PEP). The ssDSCs were fabricated using nano-crystalline $\mathrm{TiO}_{2}$ as an electron acceptor, high molar extinction coefficient ruthenium-complex dyes with wide UV-Vis absorption range as photo-sensitizer, poly(3,4-ethylenedioxythiophene) (PEDOT) as hole transporting material (HTM), and both metallic and non-metallic counter electrodes. Potential problems with PEP are un-optimised filling and poor distribution of the conjugated polymer inside the pores of the nano-structured electrode. Better pore filling and hence better photovoltaic performance has been achieved here by optimising the polymerization conditions such as illumination orientation, light intensity, dopant used, electrolyte, solvent, PEDOT growth period and $\mathrm{TiO}_{2} / \mathrm{PEDOT}$ film thickness. This increased the power conversion efficiency of the ssDSC to $3 \%$, using the ruthenium dye Z907 (cis-Bis (isothiocyanato) (2, 2'-bipyridyl-4, 4'dicarboxylato) (4, 4'-di-nonyl-2'-bipyridyl) ruthenium (II) and novel Goretex ${ }^{\circledR}$-AuPEDOT counter electrode, achieving the world's most efficient ssDSC to date employing PEP fabrication methods for this dye.

PEP optimization was also combined with non-ruthenium dye and conjugated polymers enabling efficient charge transfer. Non-ruthenium dyes have also been studied, including zinc-porphyrin (GD2 and P-10), metal-free organic indoline dye (D-149), and small organic dyes (P-257), along with differing HTMs and counterelectrodes. Liquid DSCs (Grätzel cells) were also fabricated to compare efficiencies and to determine the achievable upper limit for the ssDSCs.

Current-voltage (JV) curves were recorded and Incident Photon to Current Conversion Efficiency (IPCE) determined at various polymer pore filling and distribution levels. Imaging and electrochemical mapping techniques including Atomic Force Microscopy (AFM), Scanning Electron Microscopy (SEM) and SEMEnergy Dispersive X-ray Analyser (SEM-EDXA), have been employed to establish the morphology of the PEDOT/dyed $\mathrm{TiO}_{2}$ working electrode. HTM morphology and 
pore filling were characterised and related to the photovoltaic performance of the ssDSC. Raman spectroscopy, UV-Visible-near Infrared (UV-Vis-NIR) spectroscopy, matrix assisted laser desorption ionisation (MALDI) and mass spectroscopy were used to characterise the conductivity, doping level and the number of bis-EDOT units present in the photo-anodes.

The ruthenium dye regeneration kinetics in PEDOT-based ssDSCs were determined by Transient Absorption Spectroscopy (TAS) and compared with the common and conventional redox couple $\mathrm{I}^{-} / \mathrm{I}_{3}{ }^{-}$. The stability and reproducibility of these solar cells were also demonstrated. The thesis concludes by pointing out the next steps to be taken for the fabrication of flexible solid-state dye-sensitized solar cells. 


\section{ABBREVIATIONS}

\begin{tabular}{|c|c|}
\hline AM & Air Mass \\
\hline $\mathrm{ACN}$ & Acetonitrile \\
\hline AFM & Atomic Force Microscopy \\
\hline bis-EDOT & bis-ethylenedioxythiophene \\
\hline $\mathrm{BL}$ & Blocking Layer \\
\hline $\mathrm{CV}$ & Cyclic Voltammetry \\
\hline $\mathrm{CP}$ & Conducting Polymer \\
\hline $\mathrm{CI}$ & Constant Current \\
\hline DHB & 2, 5-Dihydroxy Benzoic acid \\
\hline DSCs & Dye Sensitized Solar Cells \\
\hline DMPII & 1,2-dimethyl-3-propylimidazolium iodide \\
\hline $\mathrm{E}_{\text {onset }}^{\text {ox }}$ & Potential at the Onset of Oxidation \\
\hline $\mathrm{EtOH}$ & Ethanol \\
\hline EDXA & Energy Dispersive X-ray Analyser \\
\hline \multirow[t]{2}{*}{ EMITFSI } & 1-ethyl-3-methylimidazolium \\
\hline & bis-trifluoromethanesulfonamide \\
\hline EDOT & Ethylenedioxythiophene \\
\hline EQE & External Quantum Efficiency \\
\hline FTO & Fluorine doped Tin Oxide \\
\hline FF & Fill Factor \\
\hline HTM & Hole Transporting Material \\
\hline $\mathrm{I}_{\mathrm{m}}$ & Current at maximum power output \\
\hline IPCE & Incident Photon to Current Conversion Efficiency \\
\hline$J_{\mathrm{sc}}$ & Short-circuit current \\
\hline $\mathrm{J}-\mathrm{V}$ & Current-Voltage \\
\hline IL & Ionic Liquid \\
\hline ITO & Indium Tin Oxide \\
\hline LiTFSI & Lithium bis-trifluoromethanesulfonamide \\
\hline MALDI & Matrix Assisted Laser Desorption Ionisation \\
\hline ML & Mesoporous Layer \\
\hline
\end{tabular}




\begin{tabular}{|c|c|}
\hline MS & Mass Spectrometry \\
\hline PCBM & {$[6,6]$-phenyl C61-butyric acid methyl ester } \\
\hline PEDOT & Poly (ethylenedioxythiophene) \\
\hline PEP & Photo Electrochemical Polymerization \\
\hline ProDOT & 3, 4-propylenedioxythiophene \\
\hline PTh & Poly (thiophene) \\
\hline PPy & Poly (pyrrole) \\
\hline P (3HT) & Poly (3-hexylthiophene) \\
\hline Q & Charge \\
\hline PET & Poly(ethylene teraphthalate) \\
\hline $\mathrm{P}_{\text {in }}$ & Incident radiation power \\
\hline PTFE & Polytetrafluoroethylene \\
\hline SEM & Scanning Electron Microscopy (SEM) \\
\hline ssDSC & Solid-State Dye-Sensitized Solar Cells \\
\hline \multirow[t]{2}{*}{ spiro-OMeTAD } & 2,2',7,7'-tetrakis-(N,N-di-p-methoxyphenyl-amine) \\
\hline & 9,9'spiro-bifluorene \\
\hline TBAP & Tetrabutylammonium perchlorate \\
\hline T-paste & Transparent paste \\
\hline TAA & Titanium diisopropoxide bis-acetylacetonate \\
\hline TBAP & Tetrabutylammonium Perchlorate \\
\hline t-BP & tertiary butyl pyridine \\
\hline THF & Tetrahydrofuran \\
\hline ToF & Time of Fight \\
\hline tris-EDOT & tri-ethylenedioxythiophene \\
\hline UV-Vis-NIR & Ultra Violet-Visible-Near Infrared \\
\hline VPP & Vapour Phase Polymerization \\
\hline $\mathrm{V}_{\mathrm{m}}$ & Voltage at maximum power output. \\
\hline$V_{\text {oc }}$ & Open-Circuit-Voltage \\
\hline vs. & Versus \\
\hline$\lambda_{\max }$ & Wavelength of Maximum Absorption \\
\hline$\eta$ & Energy Conversion Efficiency \\
\hline$\Phi_{\text {inj }}$ & Charge injection efficiency \\
\hline$\Phi_{\mathrm{col}}$ & Charge collection efficiency \\
\hline
\end{tabular}


LHE $(\lambda)$

W

w.r.t.

$\mathrm{Xe}$
Light harvesting efficiency of active material Watt

With respect to

Xenon 
TABLE OF CONTENTS

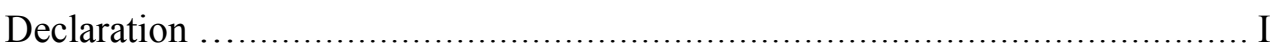

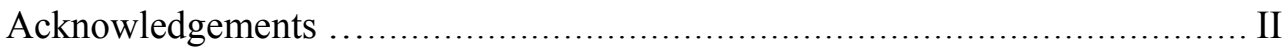

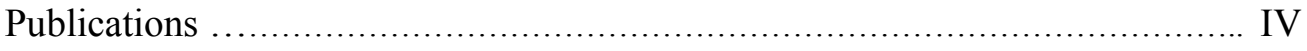

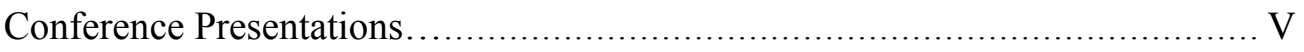

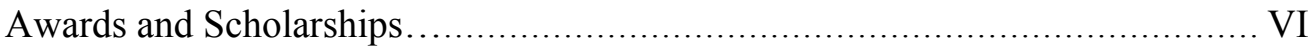

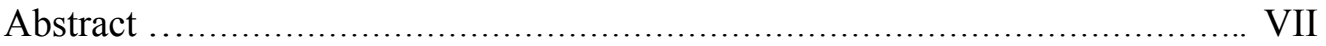

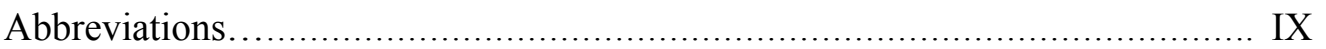

\section{CHAPTER 1}

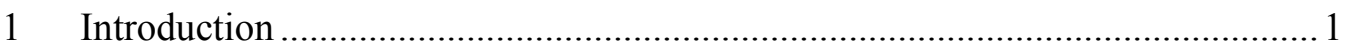

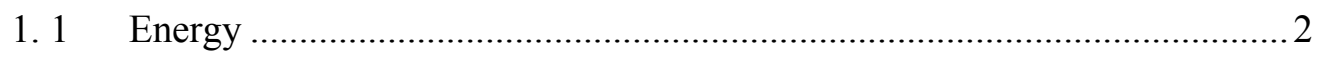

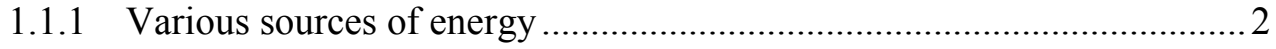

1.1.2 Initiative to meet the energy crisis ....................................................... 3

1.1.3 Cost of solar energy and market outlook …………………………….... 4

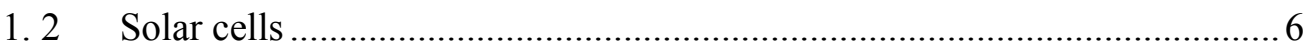

1.2.1 Different generations of solar cells ...................................................... 6

1.2.2 Introduction to non-Silicon-based solar cells ..........................................

1.2.3 Dye-sensitized solar cells (Grätzel cells) ……………………………....... 10

1.2.3.1 Operating principles and mechanism of liquid DSC...................... 12

1.2.3.2 Photo-anode requirements ............................................................... 14

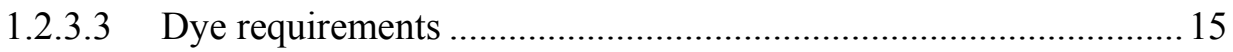

1.2.3.4 Counter electrode ………………………………....................... 17

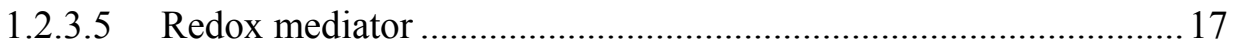

1.2.3.6 Advantages and disadvantages of DSCs ....................................... 19

1.2.3.7 Solid-state dye-sensitized solar cells ............................................. 19

1.2.3.8 Hole transporting material (HTM) .............................................. 22

1.2.3.9 Operating principles and mechanism ............................................2

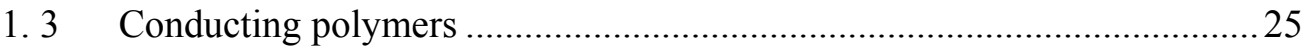

1.3.1 Introduction to conducting polymers ………………………………....2

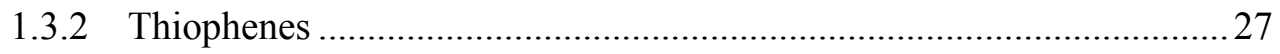


1.3.2.1 Applications of PEDOT and its mechanisim in solar cells ........... 29

1. 4 General concept of a photovoltaic device ............................................. 34

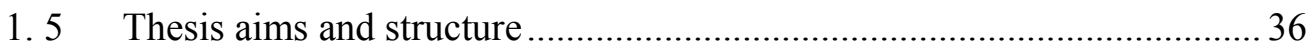

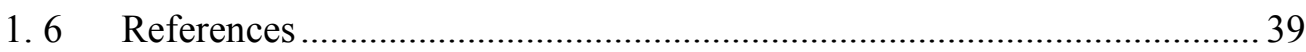

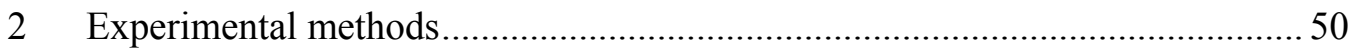

2. 1 Chemical reagents and materials.......................................................... 51

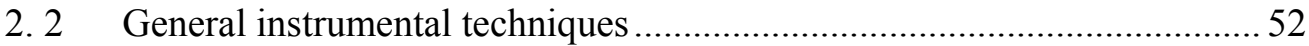

2.2.1 Electrochemical polymerization......................................................... 53

2.2.2 Photo-electrochemical polymerization.............................................. 54

2. 3 Device structure of solid-state dye-sensitized solar cells.......................... 55

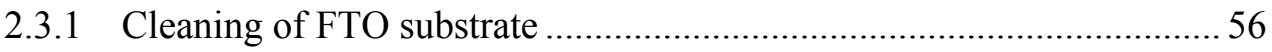

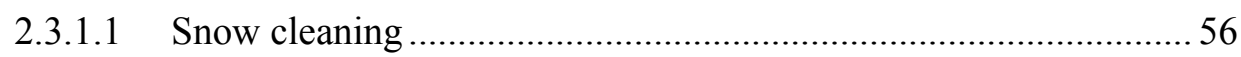

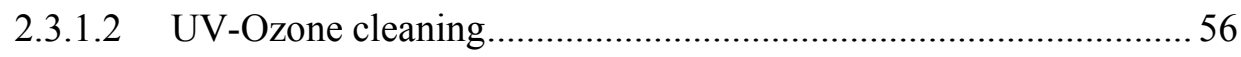

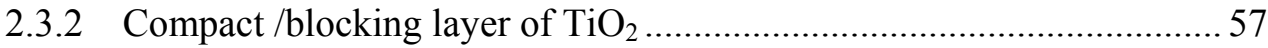

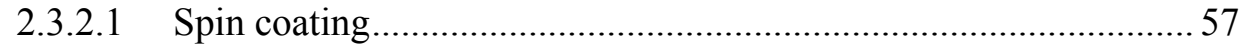

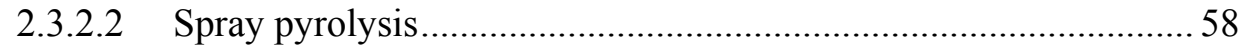

2.3.3 Preparation of $\mathrm{TiO}_{2}$ mesoporous layer.............................................5 59

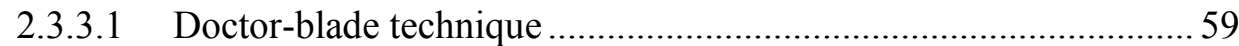

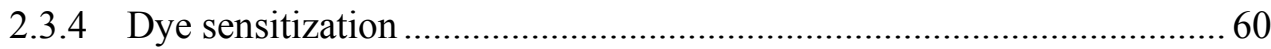

2.3.5 Impregnation of hole transporting material in dyed $\mathrm{TiO}_{2} \ldots \ldots \ldots \ldots \ldots \ldots . . . . . . .60$

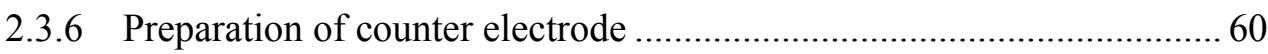

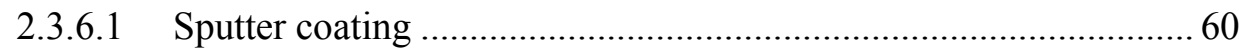

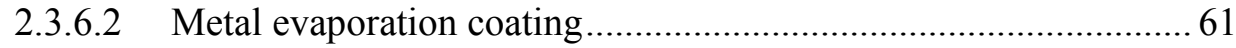

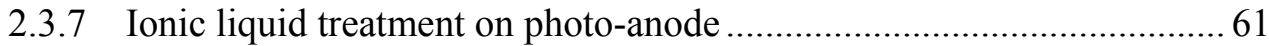

2.3.8 Assembly of the solid-state dye-sensitized solar cells ......................... 62

2. 4 Device fabrication of liquid dye-sensitized solar cells ............................ 62

2. 5 Characterisation of the photovoltaic device ..............................................6 63

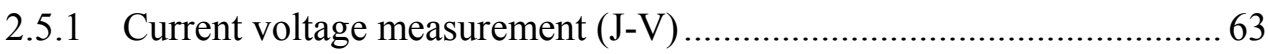

2.5.2 Incident photon to current conversion efficiency measurement ............6 64

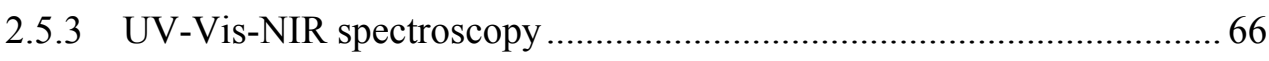

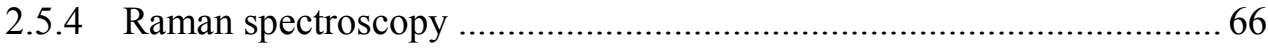

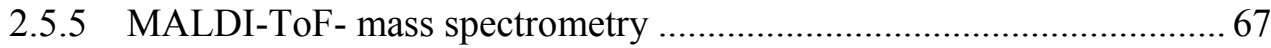

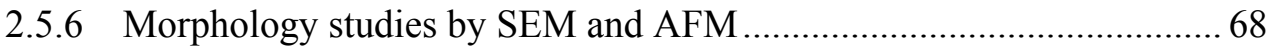


3 Fabrication of solid-state dye-sensitized solar cells ..................................... 70

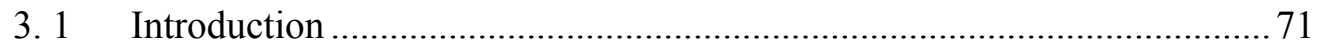

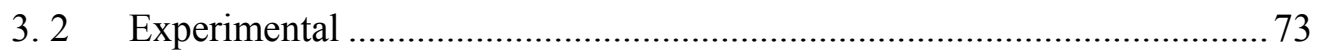

3.2.1 Calculation of HOMO-LUMO energy levels, surface coverage, and

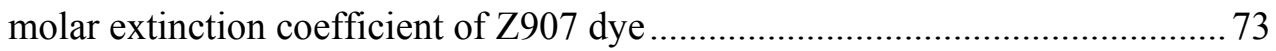

3.2.1.1 Cyclic voltammogram (CV) of Ruthenium dye.......................... 73

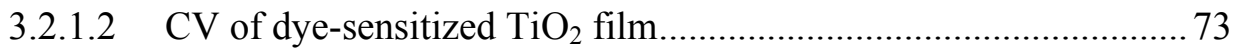

3.2.1.3 Standard Ferrocene CV ........................................................... 74

3.2.1.4 UV-Vis spectra of dyes to determine optical band gap................ 74

3.2.1.5 UV-visible spectral studies........................................................ 74

3.2.2 Fabrication of PEDOT-based solid-state dye-sensitized solar cells....... 75

3.2.2.1 Preparation of blocking layer of $\mathrm{TiO}_{2}$ on FTO glass .................... 75

3.2.2.2 Preparation of mesoporous $\mathrm{TiO}_{2}$ layer on blocking layer ............. 75

3.2.2.3 Preparation of Z907 dye solution for sensitization of working electrode 75

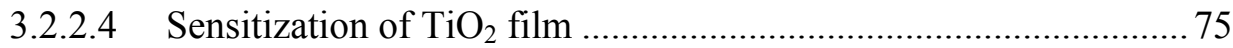

3.2.2.5 In situ photo-electrochemical polymerization of bis-EDOT ......... 76

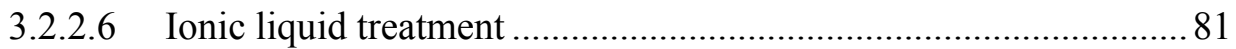

3.2.2.7 Assembly of PEDOT-based solid-state DSCs .............................. 81

3.2.2.8 Fabrication of liquid dye-sensitized solar cells (DSCs) ................ 81

3.2.2.9 Total polymerization charge ..................................................... 81

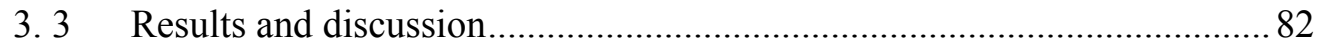

3.3.1 Calculation of HOMO-LUMO energy levels and band gaps................. 82

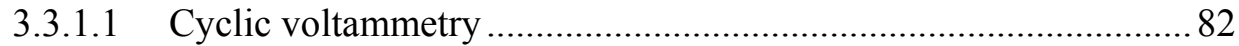

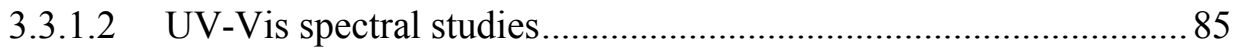

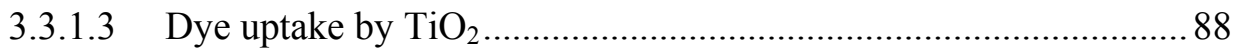

3.3.2 Photo-polymerization of EDOT ….................................................... 91

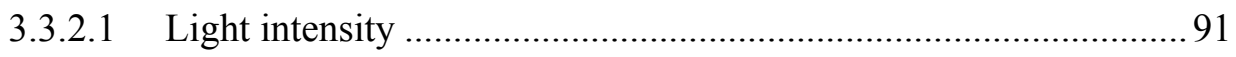

3.3.2.2 PEDOT growth time................................................................ 92

3.3.2.3 Thickness of mesoporous $\mathrm{TiO}_{2}$ layer ......................................... 93

3.3.2.4 PEDOT growth in ionic liquid ................................................... 94

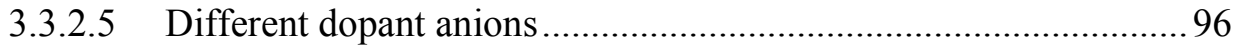

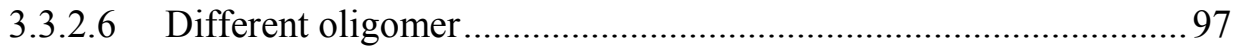


3.3.3 Solid-state dye-sensitized solar cells testing ...................................... 98

3.3.4 Photovoltaic performance measurements (JV and IPCE)................... 98 3.3.4.1 Different light intensity during photo-electrochemical

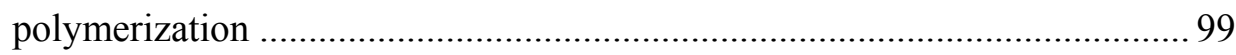

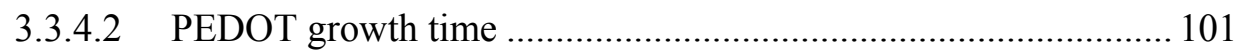

3.3.4.3 Dependence of ssDSC performance on $\mathrm{TiO}_{2}$ layer thickness ...... 103

3.3.4.4 PEDOT in ionic liquid ............................................................... 104

3.3.4.5 Influence of different dopant anions used during photoelectrochemical polymerization ............................................................ 105

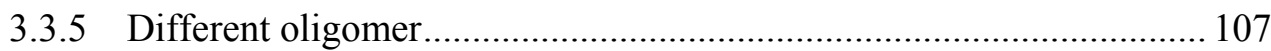

3.3.6 Dependence on light intensities of ssDSC performance ..................... 109

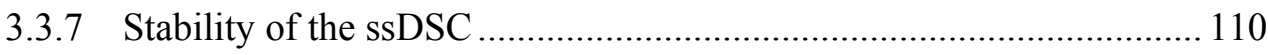

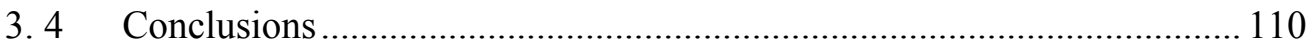

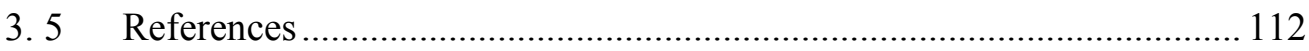

4 Morphological determination of photo-anode grown by a photo-electrochemical

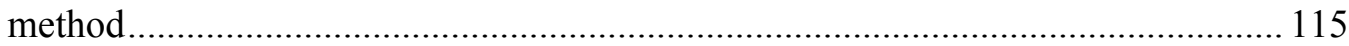

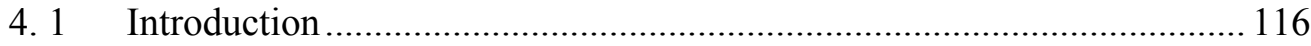

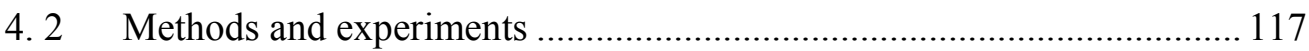

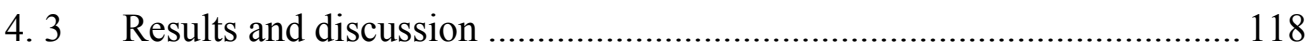

4.3.1 PEDOT optical absorption studies by UV-Vis-NIR .......................... 118

4.3.2 Raman spectral studies ............................................................... 123

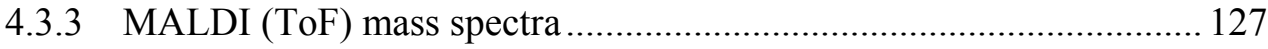

4.3.4 Morphology studies by SEM ….................................................... 128

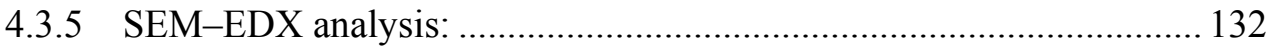

4.3.6 Morphology studies by AFM ......................................................... 134

4.3.7 Calculation of total polymerization charge ........................................ 140

4.3.8 Current-voltage (JV) and incident photon to current conversion efficiency (IPCE) measurement .............................................................. 141

4.3.9 Transient absorption spectroscopy (TAS) studies............................ 144

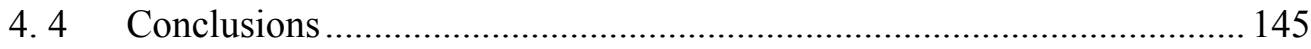

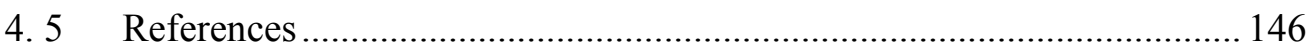

5 Novel PEDOT based counter electrodes for solid-state dye-sensitized solar cells 148 


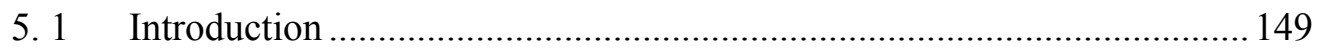

5.1.1 The importance and role of the counter electrode in ssDSCs ............. 149

5.1.2 The ideal properties of counter electrode for ssDSCs......................... 150

5.1.3 Counter electrodes that have been used to date for ssDSCs ............... 150

5.1.4 Introduction to counter electrodes developed in this chapter.............. 152

5.1.4.1 Conjugated polymer-based counter electrodes ........................... 152

5.1.4.2 Graphene-based counter electrodes........................................... 153

5.1.4.3 Goretex ${ }^{\circledR}-A u-P E D O T$ counter electrode..................................... 153

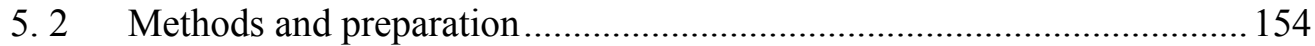

5.2.1 Sputtered Au on ITO electrodes........................................................ 155

5.2.2 Conjugated polymer-based counter electrodes ................................. 155

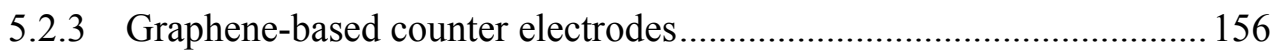

5.2.4 Goretex ${ }^{\circledR}-A u-P E D O T$ counter electrodes ...................................... 157

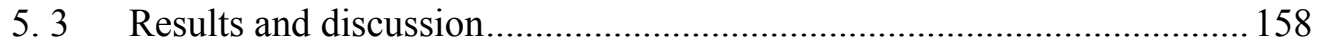

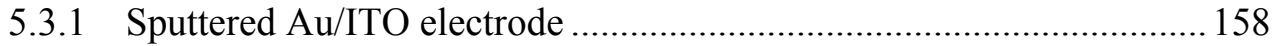

5.3.2 Conjugated polymer-based electrode ................................................... 160

5.3.2.1 Growth of PEDOT by chronoamperometry ............................... 160

5.3.2.2 Growth of PEDOT and Polypyrrole by vapour phase

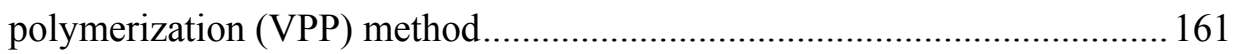

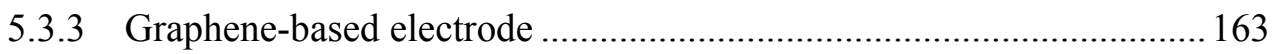

5.3.4 Goretex ${ }^{\circledR}-A u-P E D O T$ based electrode........................................... 168

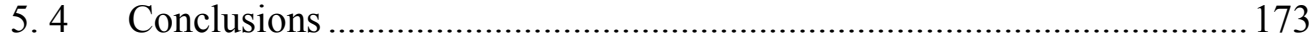

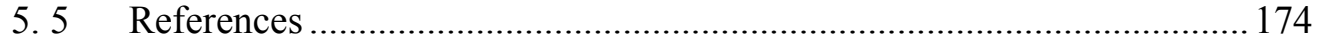

6 Non-Ruthenium based dyes for solid-state dye-sensitized solar cells ............. 178

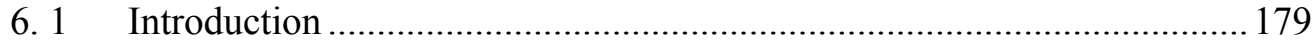

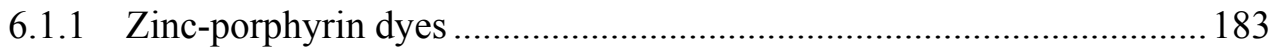

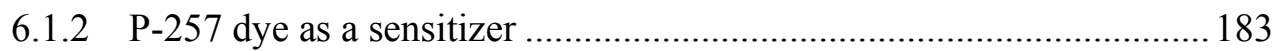

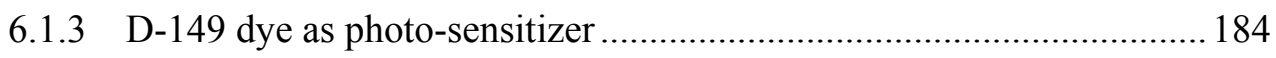

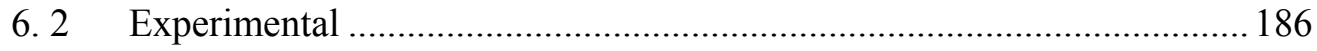

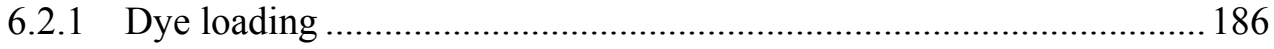

6.2.1.1 Zinc-porphyrin dye ................................................................. 186

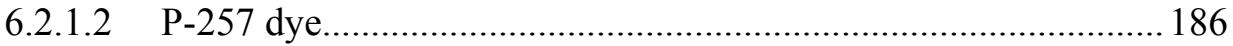

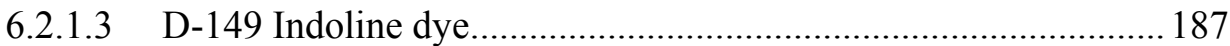


6.2.2 Calculation of HOMO-LUMO levels ............................................... 187

6.2.3 Dye desorption from the photo-anode .............................................. 188

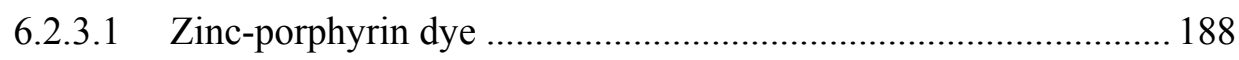

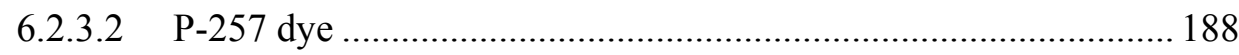

6.2.4 Preparation of working electrode by in-situ photo-electrochemical

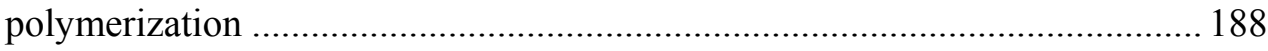

6.2.5 Device fabrication of solid-state dye-sensitized solar cells ................ 190

6.2.6 Device fabrication of liquid dye-sensitized solar cells using phenylconjugated Oligoene P-257 sensitizers …...................................................... 191

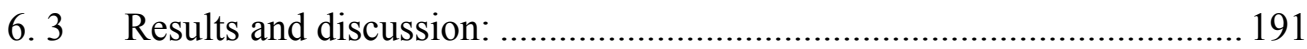

6.3.1 Dye loading of Zinc-porphyrin GD2 Dye ........................................ 191

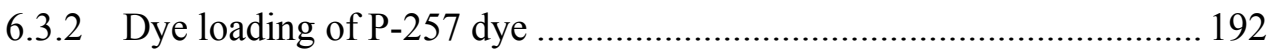

6.3.3 Calculation of the amount of $\mathrm{P}-257$ dye on $\mathrm{TiO}_{2}$ surface ................... 192

6.3.3.1 Calculation of the amount of P-257 dye desorbed from the photoanode during the photo-electrochemical deposition of PEDOT................. 194

6.3.3.2 Calculation of the amount of P-257 dye desorbed from samples prepared by PEP done under constant $200 \mathrm{mV}$ with $25 \mathrm{~mW} / \mathrm{cm} 2$ light

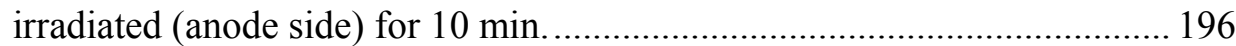

6.3.3.3 Calculation of total polymerization charge ................................. 197

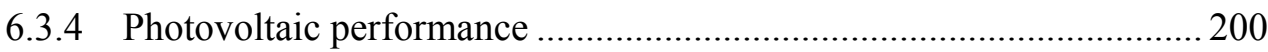

6.3.4.1 Photovoltaic (PV) performance using Zinc-porphyrin (GD2) and

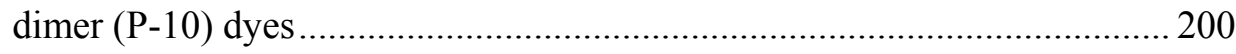

6.3.4.2 Zinc-porphyrin (GD2) dye ........................................................ 200

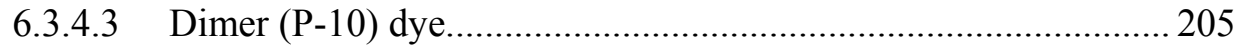

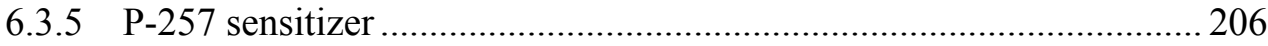

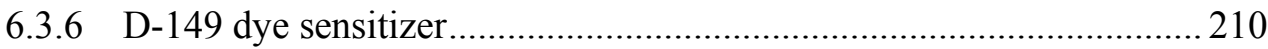

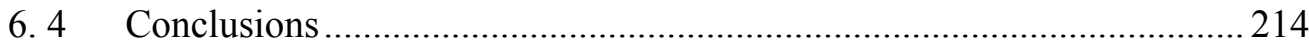

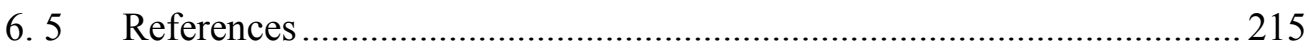

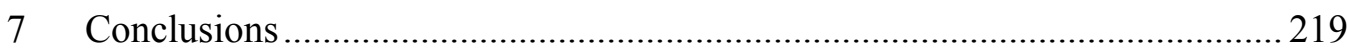




\section{INTRODUCTION}




\subsection{Energy}

\subsubsection{Various sources of energy}

There are mainly two kinds of energy sources available, namely renewable and nonrenewable energy. When the source of energy is unlimited after utilising, it is called renewable energy (such as solar energy, wind energy, tidal and wave energy, biomass, geothermal energy, hydropower energy, etc.). Utilising a non-renewable energy source leads to its depletion. A good thing about renewable energy sources is that these do not emit any kind of green house gases (pollution) to the atmosphere. By contrast, nonrenewable energy sources such as petroleum, coal, crude oil, natural gases when consumed contribute to green house gases which may affect global warming. The rapid growth of population, urbanization and industrialization of mankind has resulted in a serious problem in energy supply. The drastic decrease in non-renewable energy sources such as coal, petroleum, kerosene, diesel and uranium is a matter of world-wide concern. Fossil fuels supply $80 \%$ of the energy consumption world-wide, and oil will only last for another 40 years, natural gas 60 years and coal 200 years [1]. The energy demand by the world population is predicted to be about 13 terawatts (TW) by 2050 [2], and so innovative approaches must be identified and developed to meet this demand.

Among renewable energies from natural resources, the sun itself can provide us with 3.8 $\times 10^{23} \mathrm{~kW}$ from one hour of sunshine and it can meet the energy demand for world consumption per annum $\left(1.6 \times 10^{10} \mathrm{~kW}\right.$ in 2005) [3]. The supply of energy from the sun to earth is gigantic $\left(3 \times 10^{24} \mathrm{~J}\right.$ a year) which is 10,000 times more energy than that utilised by the world's population at present [1]. If $0.1 \%$ of the earth's surface will be covered by solar cells whose efficiency is $10 \%$, this will meet our present energy needs [1]. Prof. Mike McGhee (Material Science and Engineering, Stanford University, USA) has reported that, to meet the $13 \mathrm{TW}$ energy crisis, we need to grow the renewable energy industry very rapidly ( $35 \%$ per year) [4]. We should have enough economic stability to double the number of factories; keeping in mind that those associated materials should not run out. If we have a healthy market then we can recover our money invested and any profits can be re-invested in more factories. Various parameters have be taken into consideration to introduce the new technology, such as materials, low cost, toxicity to the 
environment, light weight, high reproducibility, ease of fabrication, aesthetics, and use of direct sunlight without loss in diffusion. The role of nanotechnology has advanced substantially to mitigate the energy crisis by fabricating various solar cells and electrical energy storage devices such as batteries and electrochemical capacitors.

\subsubsection{Initiative to meet the energy crisis}

Governments and non-government organisations should work together to realise clean, green and safe energy. In every country, the government should encourage the scientists and technologists for the development and production of renewable energy sources. Most of the developing countries need more energy because of their high population density. Socio-economics, politics, geography, population and literacy are the main key components to create awareness on energy among the populous. It is the responsibility of governments to educate their citizens to be aware of the respective energy sources and the benefits or consequences of consumption. Figure 1.1a shows the world energy consumption (13 TW in 2005) from various energy sources where oil is the largest (35\%) contributor while $24 \%$ and $22 \%$ of the energy came from coal and gas, respectively. Biomass contributed only $10 \%$ of the total energy consumed. If nuclear energy will be employed then we can meet $28 \mathrm{TW}$ by 2050 . Unfortunately, only $7 \%$ of energy is currently supplied from the nuclear sector (Figure 1.1a). If we need another 93\% more energy from this sector we have to set up nuclear $1 \mathrm{GW}$ reactor everyday (1 GW nuclear reactor per day (NREL, 2006: Approx. 13 TW, instantaneous yearly-averaged consumption rate (cf. $4.1 \times 1020 \mathrm{~J} /$ year, 2050: Estimated to be $28 \mathrm{TW}$, Perspective: 1 new $1 \mathrm{GW}$ nuclear reactor per day for the next 30 years). This would be a very difficult task. If the uranium $235\left(\mathrm{U}^{235}\right)$ isotope is used for nuclear energy for the next 20 years, we can get energy with reasonable cost, but after 20 years we need breeder reactor technology, however the use of plutonium $239\left(\mathrm{Pu}^{239}\right)$ is dangerous, requiring high safety precautions [5]. 


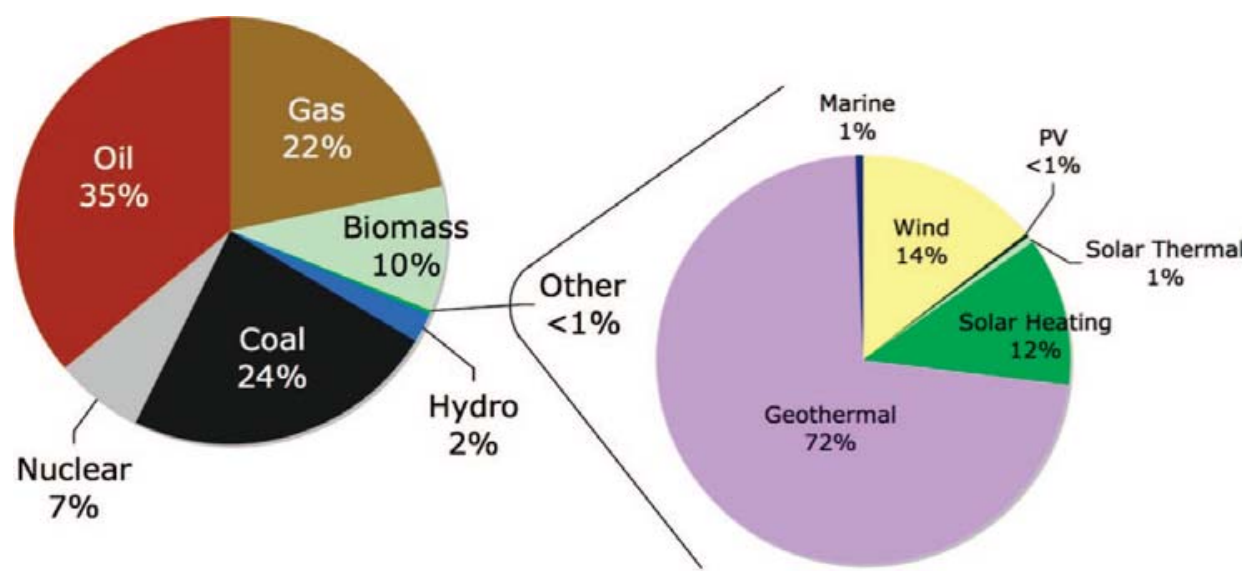

(a)

(b)

Figure 1.1: (a) A pie chart detailing the 13 TW world energy consumption from various energy sources in 2005 (b) Components of the $<1 \%$ of energy that came from alternate renewable energy resources in 2005 (source: Chemical Reviews 2010 [5]).

\subsubsection{Cost of solar energy and market outlook}

The cost of solar energy is always given by a primary unit, the watt peak (Wp). The price of a solar module is dependent on the size of the panel. Fifty to sixty percent of the high cost of silicon solar cells is attributed to the installation process and about $40 \%$ to the materials and manufacturing. The cost is also dependent on the country where it is installed. A survey reveals that the cost of solar cells is $\$ 4.00$ per watt ( $€ 3.00$ per watt) for 440 modules as of May 2010 [6]. The price varies with increase or decrease in market growth. In the US retail market, for multicrystalline silicon solar cells one has to pay $\$ 1.74$ per watt ( $€ 1.31$ per watt), whereas in Germany it is $\$ 2.07$ per watt ( $€ 1.55$ per watt) [6]. The prices also vary with different brands, certifications, warranties, technical support, etc. Figure 1.2 shows how the price of the retail module increased and decreased over the last decade both in the US and Europe. It is observed in the USA the price gradually decreased up to 2003, then increased slowly to 2006. After remaining the same for 2 years, it then again decreased from 2008 to date. The price changes in Europe since 2001 are seen to have been similar to those in the USA. 


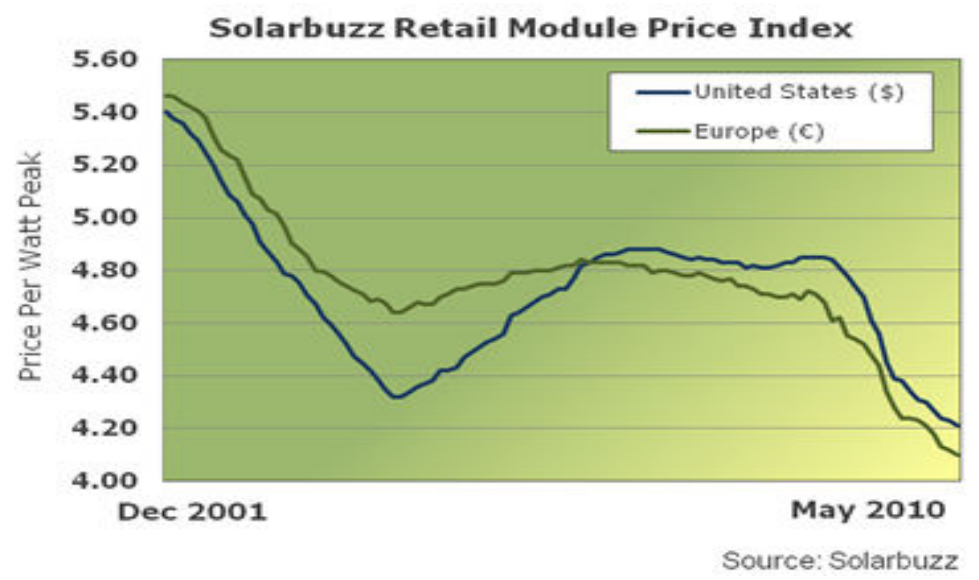

Figure 1.2: Variations of the price of silicon solar cells in the USA and Europe [6].

A comparison of annual solar energy yield cost per household at peak hrs of different countries in the world is presented in Figure 1.3. The next section discusses how one can harvest renewable energy in particular the solar energy from the sun.

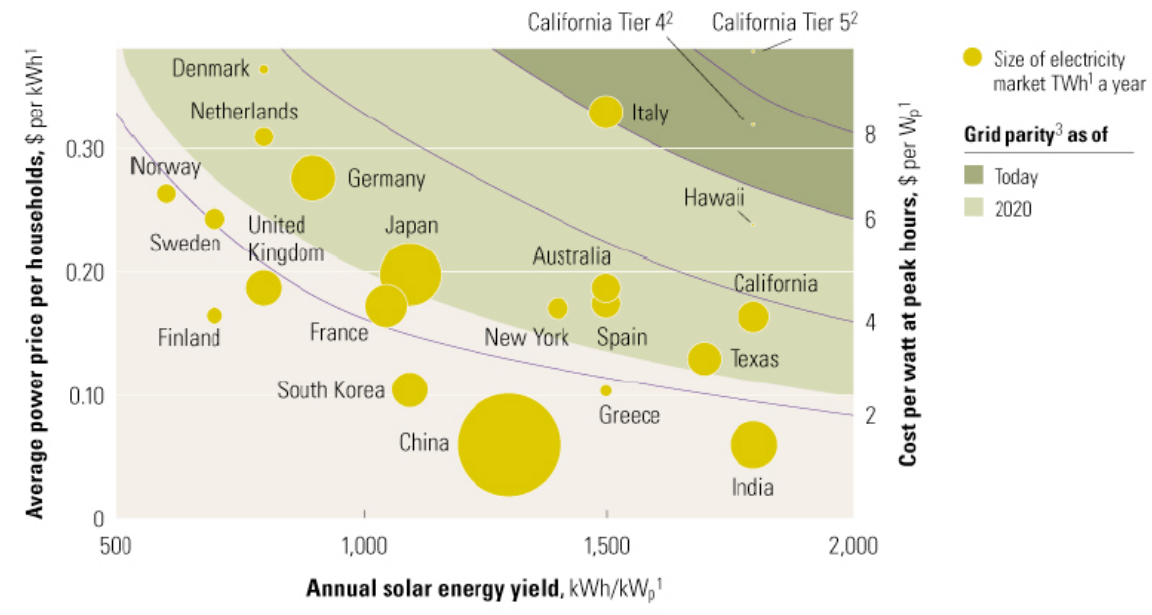

Source: CIA country files; European Photovoltaic Policy Group; Eurostat; Pacific Gas \& Electric (PG\&E); Public Policy Institute of New York State; McKinsey Global Institute analysis

Figure 1.3: Comparison of annual solar energy yield cost per household at peak hours for different countries. 


\subsection{Solar cells}

\subsubsection{Different generations of solar cells}

The first silicon solar cell was developed by the Bell Telephone Lab, USA, in 1953. An efficiency of $4.5 \%$ was achieved at that time, and after further research $6 \%$ efficiency was achieved in 1954 [7,8]. Much research has followed because silicon is safe and the second most abundant element on earth. The crystalline solar cells are known as $1^{\text {st }}$ generation solar cells. These are highly expensive because these use pure silicon. These types of solar cells are made of a single junction for extracting energy from photons and their efficiency is close to their maximum theoretical efficiency (33\%). Crystalline silicon solar cells dominate the photovoltaic (PV) market with up to $90 \%$ in 2008 [9]. This was possible because of the incredible development of silicon based semiconductor industries such as computers and electronics. The production of photovoltaic cells reached more than $7.9 \mathrm{GWp}$ (Wp is peak power tested under standard conditions) annually in world by 2008 [10]. The highest research power energy efficiency reported for these PV cells reported up to $20 \%$ however the industrial cells efficiency is limited to $15-18 \%$ [9]. A drawback of these kinds of PV cells is to get pure silicon, high temperature processing is required. Moreover, the purification of silicon is an arduous task.

Developments to reduce the cost of solar cells gave birth to $2^{\text {nd }}$ generation solar cells that are made of thin films of $\mathrm{CdTe}, \mathrm{CdSe}$, copper indium gallium diselenide (CIGS), amorphous silicon solar cells, and thin film crystalline silicon solar cells, etc. The $2^{\text {nd }}$ generation solar cells are less efficient but less expensive, and were of great interest in the 1990 's and early this century. Thin film solar cells are designed in such a way that they utilise and use less material resulting in low cost manufacturing process.

The third generation solar cells, generally an alternative to $1^{\text {st }}$ and $2^{\text {nd }}$ generation solar cells, are targeted for high efficiency and lower cost. These are tandem cells, nanostructured solar cells, and multijunction photovoltaic cells. Apart from these three generation solar cells, other kind of solar cells have been fabricated and these are liquid dye-sensitised solar cells (photo-electrochemical based), solid-state dye-sensitized solar cells, and organic solar cells [11-30]. 
Out of all the available solar cells, silicon based solar cells still dominate the photovoltaic market. Their drawback is that they are very expensive, heavier, and rigid in shape and require high temperature processing.

\subsubsection{Introduction to non-Silicon-based solar cells}

The thought of utilizing photo-electrochemistry for the application of solar energy conversion evolved after the photo-electrochemical experiment done by a young 18 year old French scientist Becquerel in 1839 [31]. He immersed two electrodes such as gold brass platinum with either silver or silver-coated with halogens ( $\mathrm{AgI}, \mathrm{Ag} \mathrm{Br}, \mathrm{AgCl}$ ) into various acidic, basic and neutral electrolytic solutions. When these electrodes were exposed into sunlight, he observed a small amount of current flow through the external circuit. This phenomenon is called the Becquerel Effect and his experiment is believed to be the first reported paper on photo-electrochemistry. The modern era of photoelectrochemistry came into existence in 1954. Before that, the Becquerel effect (photogalvanic effect) was not properly understood [32]. Since then, extensive research work has been performed on the photo-electrochemical effect and solar cells [33-34].

The low cost, high efficiency dye-sensitized solar cells (DSCs) fabricated by O'Regan and Grätzel using colloidal $\mathrm{TiO}_{2}$ to mimic the natural process of photosynthesis demonstrated a real path towards the substantial substitution of silicon solar cells [35]. The photo-sensitization of colloidal $\mathrm{TiO}_{2}$ electrodes by chlorophyll derivatives and related natural porphyrins shows high photocurrent quantum yields; approaching unity efficiency of primary charge separation as in natural photosynthesis [36]. Dye-sensitized solar cells (DSCs) are comprised of photo-anode semiconducting nanoparticles such as $\mathrm{TiO}_{2}$ [35], $\mathrm{ZnO}$ [37], $\mathrm{SnO}_{2}$ [38], $\mathrm{Nb}_{2} \mathrm{O}_{5}$ [39], optically transparent electrodes, sensitizer dyes, inorganic salts, solvents and metallic catalysts (Pt, $\mathrm{Au})$; where each component has a specific role to make the device efficient. However, in this type of solar cell there are some disadvantages involving stability, leakage of solvent and sealing [35, 40-42]. 
To replace the volatile liquid electrolyte in DSCs, solid-state DSCs were fabricated by using either solid-state or quasi solid-state hole conductors. These are p-type semiconductor or organic hole conductor materials, ionic liquid electrolyte or polymer electrolyte. The selectivity of a p-type semiconductor in solid-state DSCs should be such that it can transfer the holes from photo-sensitizer after the dye injects electrons into conduction band of $\mathrm{TiO}_{2}$. That is, the upper edge valence band of hole transporting material (HTM) must be above that of the ground state energy level of the sensitizer. These materials should be transparent in the visible region, not dissolve the photosensitizer, and able to deposited in the nanoporous network of dyed $\mathrm{TiO}_{2}$. This approach is suitable to get rid of problems such as sealing, leakage and corrosion in DSCs. However, these cells show less efficiency $(\sim 5 \%)$ compared to DSCs $(\sim 11 \%)$.

Inorganic p-type semiconductor made of copper, such as $\mathrm{CuI}, \mathrm{CuSCN}$, and $\mathrm{CuBr}$, have also been used as hole conductors for solid-state DSCs. These materials can be deposited either by casting from solution or vacuum deposition. Tennakone et al. [43] first reported using $\mathrm{CuI}$ as a HTM for solid-state DSCs and the efficiency of these kind of solar cells was limited to $2.4 \%$. Taguchi et al. [44] and Kumar et al. [45] fabricated a solid-state DSC with $\mathrm{MgO}$ coated $\mathrm{TiO}_{2}$ nanoporous layer and $\mathrm{CuI}$ as $\mathrm{HTM}$, which resulted in better stability and enhanced power conversion efficiency. Other high band gap p-type inorganic semiconductors such as $\mathrm{SiC}$ and $\mathrm{GaN}$ are not good HTMs because they will degrade the dye under high temperature and vacuum deposition techniques.

Quasi solid-state dye-sensitized solar cells have also been developed. Extensive study has been done on different kinds of quasi-solid-state DSCs using gel electrolytes such as polymer electrolyte and polymer gels. Cao et al. used a mixture of sodium iodide, ethylene carbonate, propylene carbonate and polyacrylonitrile; but this kind of device has drawbacks because the gel is unstable which causes low conductivity and solvent leakage, and alters the sealing condition with high temperature [46-47]. Less study has been done on inorganic-organic hybrid solar cells [48-51].

Organic solar cells have also been developed. The cell consists mainly of two organic materials that are electron donating and electron accepting, sandwiched between two 
conducting contacts such as indium tin oxide (ITO) and a low work function metal such as $\mathrm{Ca}, \mathrm{Al}$ or $\mathrm{Mg}$. The best feature of this design architecture is that the charge generation efficiency is relatively independent of the bias voltage. A fill factor (FF) of 0.65 and $1 \%$ power efficiency have been achieved by using thin films of a two layer organic solar cell of copper phthalocyanine and perylene tetracarboxylic derivatives [51-52].

Organic solar cells are attractive because they can be fabricated easily in a cost effective way, they are light weight and flexible; and their performance can be manipulated by molecular designing and synthesis of new polymer or organic semiconductors. The efficiency of polymer solar cells, however, is low compared to silicon solar cells and DSCs because of the mismatched spectrum between the photo response of the device and the solar radiation, the low free charge carrier's mobility, and inefficient charge collection by the electrode. Stability is another problem for commercialization [53-54]. Various types of organic solar cells such as single layer (p-type schottky device), bilayer heterojunction, diffuse bilayer heterojunction and bulk heterojunction solar cells [55-58] have been fabricated.

Another advance is in the development of a tandem solar cell that consists of a multilayer structure equivalent to two photovoltaic cells in series, so that the open circuit voltage $\left(V_{\text {oc }}\right)$ is increased as the sum of the $V_{\text {oc }}$ of individual cells. They are made of bulk heterojunction materials such as semiconducting polymers and fullerene derivatives. A maximum efficiency of $6.7 \%$ has been reported [36-37]. Thin film solar cells [59] and various organic-inorganic hybrid nanocomposite solar cells [60-63] have been fabricated but the efficiencies of these devices are also relatively low compared to others discussed above.

Various solar energy technologies are presented in Figure 1.4. It is observed that improvements in solar cell efficiency in the laboratory have occurred over the last 30 years. Figure 1.4 shows that the progress in crystalline silicon solar cell is better than for any single junction GaAs and multi junction concentrators, as far as efficiency and cost are concerned. The efficiencies of DSCs have been also increased from 2002 to the present. 


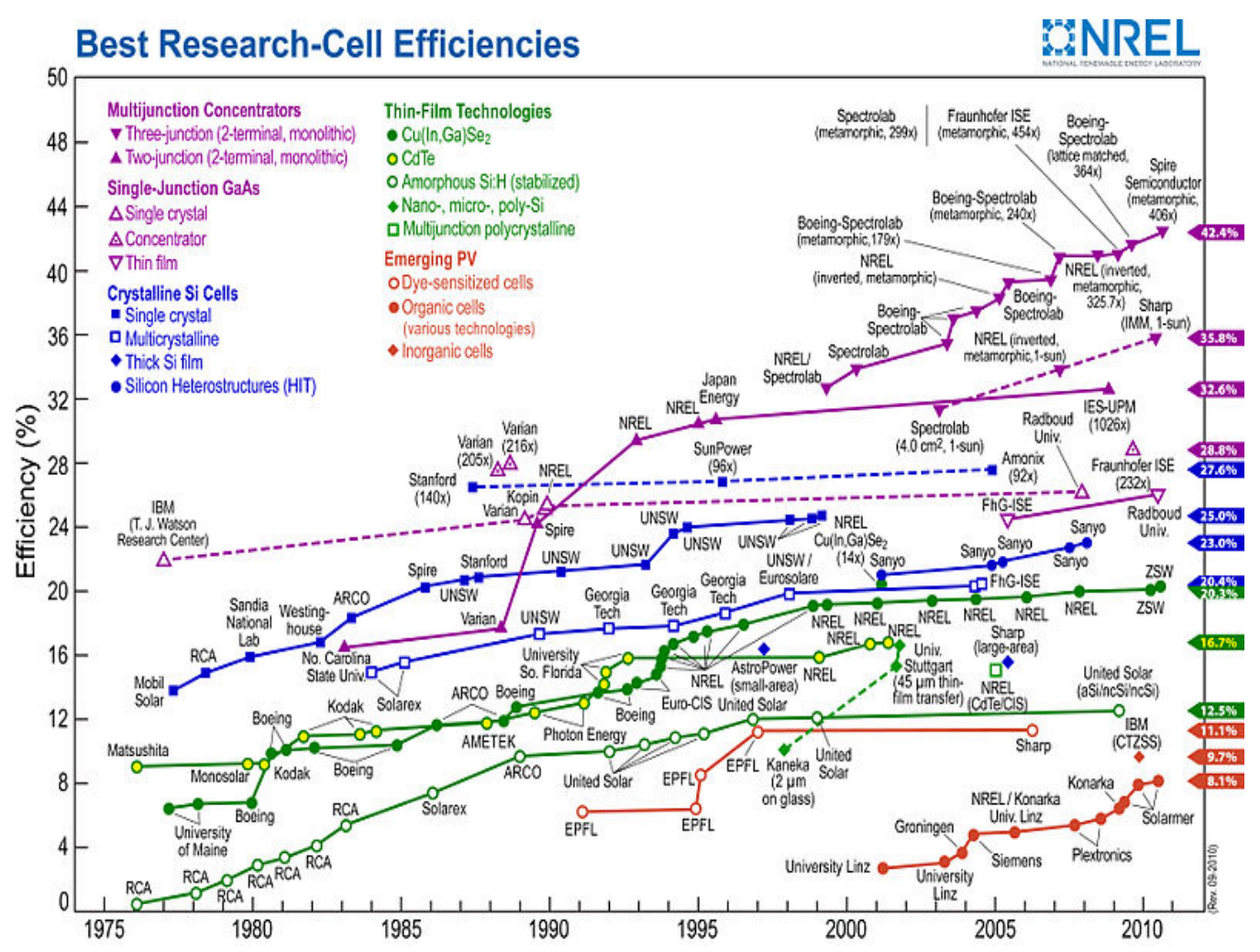

Figure 1.4: Historical trends in cell efficiency for different photovoltaic technologies (source: NREL, USA).

\subsubsection{Dye-sensitized solar cells (Grätzel cells)}

The low cost and ease of fabrication of dye-sensitized solar cells have created much interest [64], as seen from the rapid rise in publications and patents since 2000.

The basic configuration of dye-sensitised solar cells is given in Figure 1.5. The key components in dye-sensitized solar cells are the photo-anode, redox mediator, and counter electrode. The selection of these materials depends on their material properties being suitable within the DSC.

1. A thin blocking or compact layer $(\sim 100 \mathrm{~nm})$ of $\mathrm{TiO}_{2}(\sim 5 \mathrm{~nm})$ is coated on FTO substrates. These can also be done on other available various transparent 
conducting glass (TCO) or plastic substrates such as indium doped tin oxide (ITO) or flexible ITO-coated polyethylene terephthalate (PET). These transparent conducting oxides (TCO) electrode used for current collection.

2. The mesoporous oxide layer $(\sim 10 \mu \mathrm{m})$ is comprised of $\mathrm{TiO}_{2}$ nanoparticles coated on blocking layer. These mesoporous $\mathrm{TiO}_{2}$ layers are sintered for better internecking among $\mathrm{TiO}_{2}$ particles $(\sim 20-30 \mathrm{~nm})$.

3. The surface of the nanocrystalline oxide layer is coated with a monolayer of various charge transfer dyes. When the light falls on the dyed $\mathrm{TiO}_{2}$, photoexcitation of the sensitizer happens and the injection of electrons takes place into the conduction band of $\mathrm{TiO}_{2}$, leaving the sensitizer in its oxidised state. The photo-sensitizer can be returned to its original ground state by electron transfer from the redox mediator (iodide/ triiodide).

4. The redox mediator $\left(\mathrm{I}^{-} / \mathrm{I}_{3}^{-}\right)$is dissolved typically in an organic solvent.

5. The oxidation of $\mathrm{I}^{-}$leads to the formation of $\mathrm{I}_{3}{ }^{-}$and an electron goes to the cathode (platinum coated ITO substrate) where the regenerative cycle completes by electron transfer to reduce $\mathrm{I}_{3}^{-}$to $\mathrm{I}^{-}$.

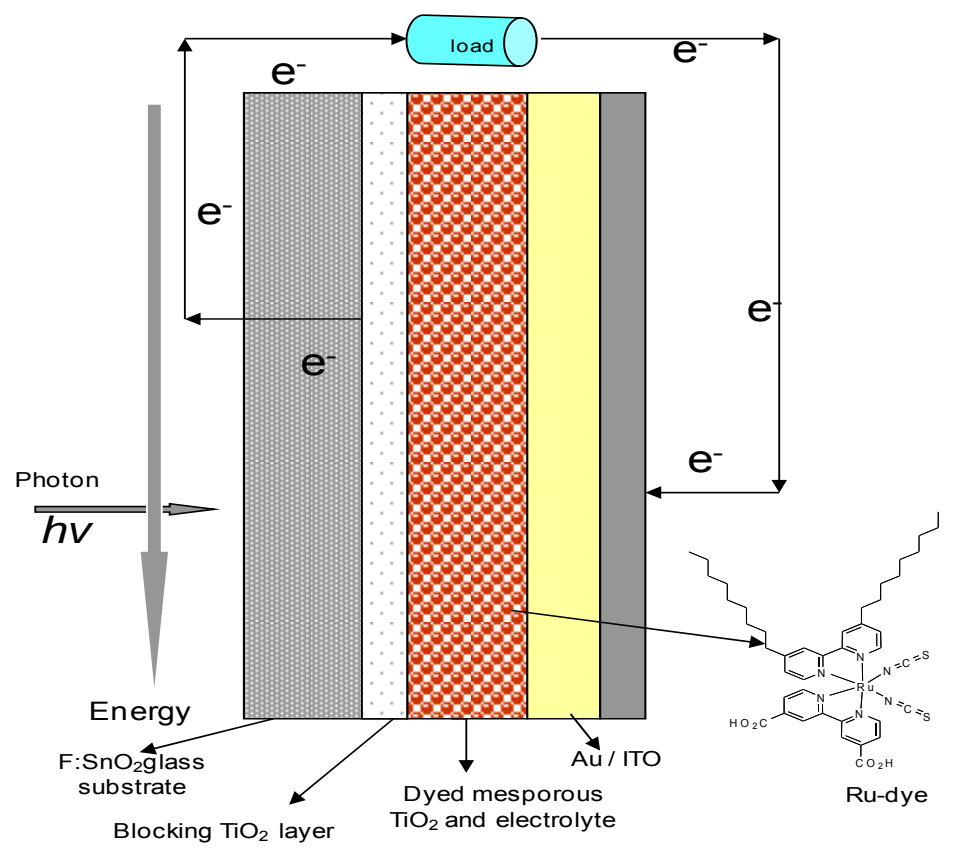

Figure 1.5: Schematic of the structure of a dye-sensitized solar cell. 


\subsubsection{Operating principles and mechanism of liquid DSC}

Here, we will discuss the operating principle and kinetic data for the different electron transfer that take place at the various interfaces $\left(\mathrm{TiO}_{2} /\right.$ sensitizer /redox couple) in DSCs. This will illustrate the limitations of the photovoltaic performance within the devices. One has to understand the following electron transfer steps:

1. Electron injection and excited state decay

2. Regeneration of the oxidized dyes

3. Electron transport through the mesoporous oxide film

4. Recombination of electrons in the semiconductor with oxidised dye or redox mediator

5. Reduction of electron acceptors in the redox mediator at the counter electrode.

The basic electron transfer process occurring with DSCs is presented in Figure 1.6 [64] and illustrated below.

1. When light falls on the cell, the dye oxidises and injects electrons into the conduction band of $\mathrm{TiO}_{2}$. For Ru-complex dyes electron injection from the excited dye into the conduction band of $\mathrm{TiO}_{2}$, shown Figure 1.6, is believed to be ultrafast $\left(10^{10}-10^{15} \mathrm{~s}^{-1}\right)$ [64]. For effective function the decay of excited dye to ground state must be slower than the injection time; decay times of 20-60 ns are typical for Ru-complex dyes.

2. The dye or photo-oxidized dye can be regenerated, via reduction of oxidised dye by the redox mediator $\left(10^{8} \mathrm{~s}^{-1}\right)$.

3. The photo-oxidised dye captures an electron from iodide $\left(\mathrm{I}^{-}\right)$from the electrolyte and $\mathrm{I}^{-}$ oxidises to triiodide. This happens in same time scale.

4. Triiodide $\left(\mathrm{I}_{3}{ }^{-}\right)$regains its electron from the counter electrode when the cell is externally connected with the load.

5. The charge transfer through the nanocrystalline $\mathrm{TiO}_{2}$ network occurs by a diffusion (or percolation) process $\left(10^{3}-10^{0} \mathrm{~s}^{-1}\right)$. The electron goes from one crystal to another by a hopping method.

6. The recombination of photo-injected conduction band electrons with the oxidised form of the electrolyte redox couple $\left(\mathrm{I}_{3}{ }^{-} / \mathrm{I}^{-}\right)$ions occurs in a different time scale (from 
microsecond to millisecond) depending upon the concentration of electrons in the semiconductor and the intensity of light.

6. The low charge transfer resistance $\left(<1 \Omega \mathrm{cm}^{2}\right)$ of the counter electrode always favours better reduction of the electron acceptor $\left(\mathrm{I}_{3}{ }^{-}\right)$in the redox mediator, resulting in better long term stability and better performance in the DSC [64].

7. The voltage developed within the cell under illumination condition is due to the energy difference between the quasi-Fermi levels of the electron in $\mathrm{TiO}_{2}$ and the electrochemical potential (redox or Nernst) potential of the redox mediator.

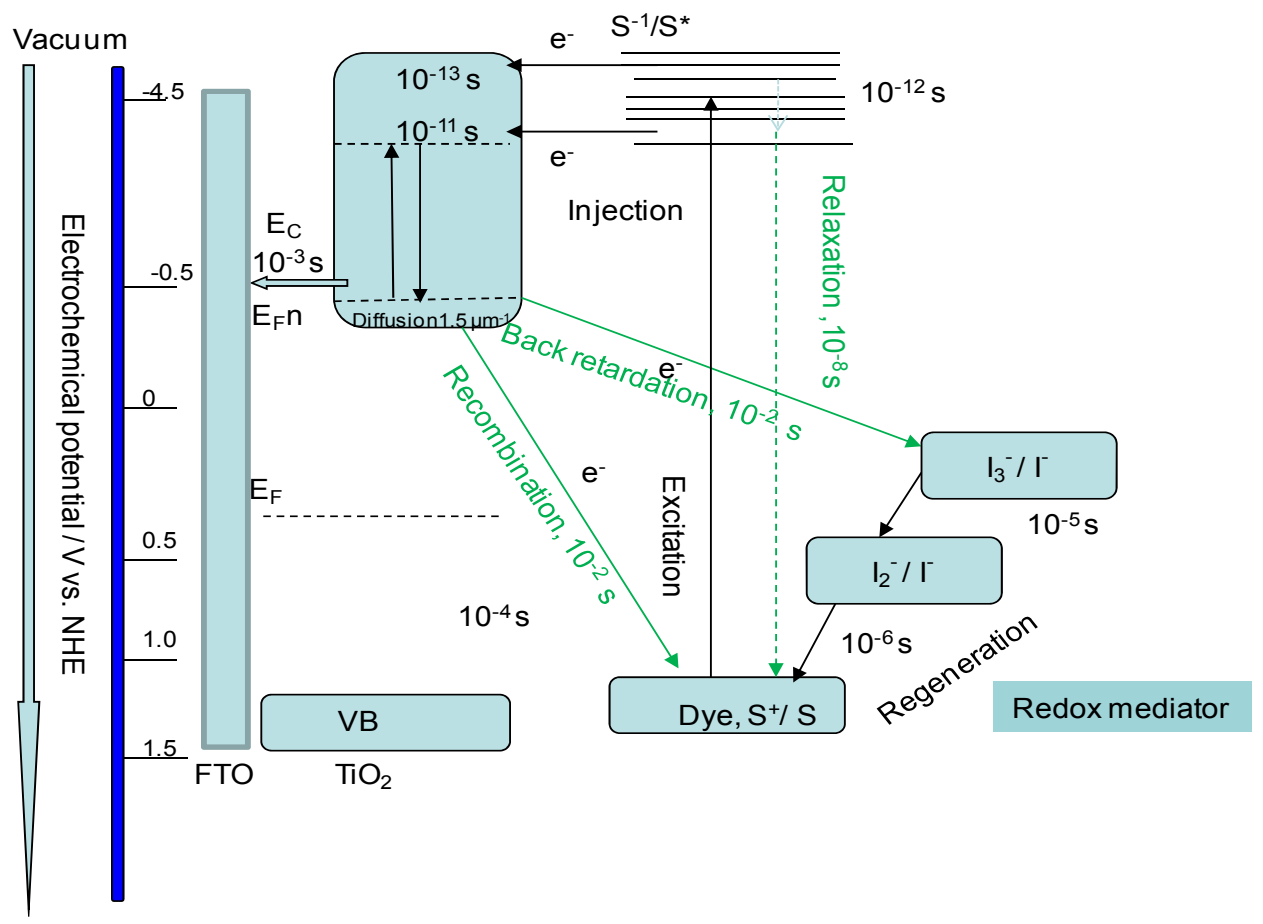

Figure 1.6: Operating principles of dye-sensitized solar cells (DSCs) under working conditions in one sun when $\mathrm{Ru}-$-dye and standard redox couple $\left(\mathrm{I}^{-} / \mathrm{I}_{3}{ }^{-}\right)$are used. All recombination paths are assigned with green arrows.

Before selecting the dye, redox mediator and counter electrode, one has to understand light absorption via metal-to-ligand charge transfer (MLCT), charge separation, charge transport, ion transport in the redox mediator, recombination, and their interfacial 
kinetics. To study this entire phenomenon we have to characterise this device by ultramodern spectroscopic techniques such as transient absorption spectroscopy and step light induced modulated photo-voltage and photo-current (SLIM-PCV) measurements.

\subsubsection{Photo-anode requirements}

A photo-anode comprises a transparent conducting oxide (TCO) glass substrate (e.g. either F: $\mathrm{SnO}_{2}$ or In: $\mathrm{SnO}_{2}$, ) coated with large band gap semiconductor materials such as $\mathrm{TiO}_{2}$ [35], $\mathrm{SnO}_{2}$ [38], $\mathrm{Nb}_{2} \mathrm{O}_{5}$ [39] or $\mathrm{ZnO}$ [37] anchored with a monolayer of charge transfer dye. The highly conductive, less resistive, less transmitting FTO/ITO is used for the photo-anode. The porosity of the $\mathrm{TiO}_{2}$ film is roughly 50-60\%. Higher porosity films, more than $60 \%$, decreases the charge collection efficiency in the device. Apart from these oxides, ternary oxides such as $\mathrm{Zn}_{2} \mathrm{SnO}_{4}$ and $\mathrm{SrTiO}_{3}$, and the core-shell like structure of $\mathrm{ZnO}$ coated with $\mathrm{SnO}_{2}$ [64-67], have been investigated for photo-anodes in the fabrication of DSCs. Researchers have also studied on the various morphology and structure of the semiconductor for the electrode, and investigated how these factors influenced the efficiency of the DSC [68]. $\mathrm{TiO}_{2}$ has several crystalline forms such as anatase, rutile and brookite. Among these three forms, brookite is very difficult to synthesise. Although rutile has the smallest band gap $(3.0 \mathrm{eV})$ and is thermodynamically stable, it is not completely transparent in the UV-Vis range. So, the anatase form of $\mathrm{TiO}_{2}$ (band gap 3.2 $\mathrm{eV}$ ) has been extensively utilised for the fabrication of DSCs.

Nano-crystalline anatase $\mathrm{TiO}_{2}$ is a semiconductor oxide material (absorbs at $388 \mathrm{~nm}$ ), and has a higher conduction band energy $\left(E_{c}\right)$ which enhances the Fermi level as well $V_{\text {oc }}$ in DSCs. The anatase form of $\mathrm{TiO}_{2}$ is highly transparent in the solar spectrum region and stable both towards photo-corrosion and temperature. Moreover, the synthesis of the nanostructured anatase $\mathrm{TiO}_{2}$ particles could be done by various easy wet chemical methods such as colloidal, sol-gel, peptization, hydrolysis, precipitation, as well as other methods such as micelle and inverse micelle, hydrothermal, sonochemical, electrodeposition, physical vapour deposition, microwave deposition technique and direct 
oxidation [64]. Other methods are also employed such as spray pyrolysis, sputtering and spin coating in the laboratory for this purpose.

For the photoanode, the mesoporous $\mathrm{TiO}_{2}$ layer is made in the following way to enhance the PV performance of the DSC.

1. A compact/ blocking $\mathrm{TiO}_{2}$ layer $(\sim 50-100 \mathrm{~nm})$ is coated on FTO substrate to prevent the contact between the redox couple in electrolyte and the FTO. Coating of this layer is done by either spray pyrolysis or spin coating.

2. The use of this blocking layer is to prevent the ohmic contact between redox mediator and FTO glass. The FTO inevitably has small pores which will allow the redox mediator into it, thus creating a short circuit.

3. A $10 \mu \mathrm{m}$ thickness of mesoporous $\mathrm{TiO}_{2}(\sim 20 \mathrm{~nm})$ layer is used for the light absorption layer. This layer is made by either doctor blading or screen printing. The layer provides a better adsorption of dye onto $\mathrm{TiO}_{2}$. It also helps in transporting electrons to the substrate.

4. A $3 \mu \mathrm{m}$ thick layer of porous $\mathrm{TiO}_{2}(400 \mathrm{~nm})$ on top of the mesoporous $\mathrm{TiO}_{2}$ layer is used as a scattering layer.

The dye-sensitized flat surface of $\mathrm{TiO}_{2}$ always absorbs less light, but dye-sensitized nanocrystalline $\mathrm{TiO}_{2}$ film generates more than 1000 times the photocurrent compared to its counterpart single crystalline film. The nanocrystalline film absorbs the sensitizers more effectively as it has more surface area than flat surface. The nanocrystalline film when anchored with a monolayer of dye enhances light harvesting by 1000 times because of its high internal surface area. The nano-structured film is prepared by either doctor blading or screen printing followed by high temperature $\left(450^{\circ} \mathrm{C}\right)$ sintering. A nanoparticle based electrode always increases the photoactive interface compared to its larger domain size film.

\subsubsection{Dye requirements}

A variety of dyes have been synthesised for application in dye-sensitized solar cells. The most popular dyes to date are ruthenium based dyes (N719, Z907 and N3) [69-71], zinc 
porphyrin dyes $\left(\mathrm{GD}_{2}\right.$, dimer (P-10) [72-73], indoline dye [74], metal-free organic dyes (indoline, $\pi$-conjugated phenolgene) [75], phthalocyanines [76], coumarin dye [77], tetrahydroquinoline dyes [78], triarylamine dyes [79], carbazole dyes [80], perylene dyes [81], squaraines [82] dyes. The transition metal based complex dyes have anchoring ligands which are solely responsible for their adsorption onto the semiconductor surface and also to chromophoric groups. Ruthenium based dyes are commonly used in dye sensitized solar cells. Ruthenium dyes contain polypyridyl ligands and metals such as Ru or Os having different ancillary ligands of halides groups, cyano $(-\mathrm{CN})$, thio-cyano ($\mathrm{SCN}), \mathrm{H}_{2} \mathrm{O}$, etc. The excitation of the $\mathrm{Ru}$ complex occurs via photon absorption and is of a metal-to-ligand charge transfer (MLCT) depending on the wavelength of light. One should have a clear understanding of its absorption, energetics, kinetics, stability and interfacial properties before choosing a dye for application in DSCs.

The following conditions should be met for a dye to be an effective photo-sensitizer for solar cells:

1. The intended dye should have a high molar extinction coefficient covering as much of the solar spectrum as possible and absorption goes up to $900 \mathrm{~nm}$.

2. Dyes should have anchoring groups such as $-\mathrm{COOH},-\mathrm{H}_{2} \mathrm{PO}_{3},-\mathrm{SO}_{3} \mathrm{H}$ to bind the dye strongly onto the semiconductor (e.g. $\mathrm{TiO}_{2}$ and $\mathrm{ZnO}$ ) surface.

3. The oxidation potential of the dye must be more positive than the redox potential of the electrolyte for dye regeneration.

4. The LUMO level of the dye should allow efficient electron transfer from the photo-excited dye to the semiconductor.

5. Thermal, electro-chemical and photo stability of the dye are important parameters under operational conditions.

6. Ideally, the dye should have bulky groups to prevent aggregation. Dye aggregation could be prevented either by optimising the molecular structure of the dye or by adding co-adsorbent in the dye solution when semiconductor oxide material is sensitised with dye. 
These kinds of DSCs can have a variety of colours depending upon the photo-sensitizers used, and could be used for enhancing the aesthetics of a building where the PV modules are installed. If we can synthesise a dye which absorbs sun light beyond the visible or near-IR, then more photons can be collected within the near-IR spectrum.

\subsubsection{Counter electrode}

A catalyst of a thin layer of metal coated on TCO substrate is used as counter electrodes for DSCs when $\mathrm{I}^{-} / \mathrm{I}_{3}{ }^{-}$is used as redox mediator. The low amount of metallic catalyst on the counter electrode enhances fast reaction kinetics for the redox mediator. FTO itself is a poor counter electrode due to its high charge transfer resistance $\left(>10^{6} \Omega \mathrm{cm}^{2}\right)[64]$ in $\mathrm{I}^{-}$ $/ \mathrm{I}_{3}{ }^{-}$mediator. This metal catalyst coating can be prepared by various chemical, physical and electrochemical methods.

Several other counter electrodes have also been examined for DSCs such as carbon based graphite [83] carbon powders [84], and single wall carbon nanotubes [85] and graphene [86] have also been used in DSCs. Conducting polymer based counter electrodes such as poly (3, 4-ethylenedioxythiophene), polyaniline and polypyrrol and poly (3, 4propylenedioxythiophene) (ProDOT) [87] are found to be suitable within DSCs. Among all counter electrodes, Pt on ITO has been found to be the most promising electrode for dye-sensitized solar cells. There is still much debate on the stability issues of this $\mathrm{Pt}$ counter electrode towards the solvent and redox couple $\left(\mathrm{I}^{-} / \mathrm{I}_{3}{ }^{-}\right)$.

\subsubsection{Redox mediator}

The redox couple must have low toxicity for the environment and be inexpensive for mass production of devices. As for the redox mediator, its electrochemical potential must be well matched with the quasi Fermi energy level of the semiconductor to maximise the photovoltage. It should be chemically and thermally stable under solar cell operation. It should not absorb light in the UV-Vis and near-IR range. Moreover, the redox mediator should not react chemically with any of the components of the DSC. 
The standard electrolyte used for dye-sensitized solar cells is the $\left(\mathrm{I}^{-} / \mathrm{I}_{3}{ }^{-}\right)$redox couple in an organic solvent. Apart from these key components, one should look into other matters such as sealing and evaporation of electrolyte from the device. In the redox couple $\mathrm{I}^{-} / \mathrm{I}_{3}{ }^{-}, \mathrm{I}^{-}$ is an electron donor (reductant) whereas $\mathrm{I}_{3}{ }^{-}$is an electron acceptor (oxidant). The various steps involved in redox mediator are given below.

Various steps involves in the reduction of oxidised dye by iodide:

1. $\mathrm{S}^{+}+\mathrm{I}^{-} \rightarrow\left(\mathrm{S}^{\cdots} \mathrm{I}\right)$

2. $\left(\mathrm{S}^{\cdots} \mathrm{I}\right)+\mathrm{I}^{-} \rightarrow\left(\mathrm{S}^{\cdots} \mathrm{I}_{2}^{-}{ }^{-}\right)$

3. $\left(\mathrm{S}^{\cdots} \mathrm{I}_{2}^{-}\right) \rightarrow \mathrm{S}+\mathrm{I}_{2}{ }^{-}$

4. $2 \mathrm{I}_{2}^{-} \rightarrow \mathrm{I}_{3}^{-}+\mathrm{I}^{-}$

Before selecting any redox mediator, one should keep in mind that the solvent must be able to dissolve the mediator in both its oxidised and reduced forms. It must have a high dielectric constant and low viscosity for effective transfer of both mass and charge. The solvent must have low volatility for longer operation of DSC.

Solvent must be a good solvent for dissolving additives (e.g. 1-methylbenzimide or 4-tertbutylpyridine) and other components tetrapropylyammonium iodide or iodine) of redox mediator, but must not dissolve either the absorbed dye or oxide material of the photoanode.

Two types of solvents that are suitable for the redox mediator for DSC are either polar organic solvents or ionic liquids. The choice of solvent should also avoid problems in sealing the cell and leakage of redox couple. Various solvents have been used in redox couples to date: tetrahydrofuran (THF), dimethyl sulfoxide (DMSO), ACN (acetonitrile), valeronitrile (VN), organic carbonates (e.g. ethylene carbonate), and mixtures of organic carbonate with ACN and 3-methoxypropionitrile (MPN) and various alcohols. 


\subsubsection{Advantages and disadvantages of DSCs}

The advantage of DSCs is that the photovoltaic performance increases with increase of temperature, unlike silicon solar cells. We can modulate in a bifacial configuration where diffusion of light is possible in between counter and working electrode, which is not possible in silicon based solar cells. These kinds of solar cells do not need ultra pure materials because the conduction mechanism is dependent on the majority carrier transport. These DSC also provide the possibility of fabricating light-weight, transparent, flexible devices with multi-colours by choosing appropriate materials for different purposes. In addition, they are cost effective as compared to conventional silicon solar cells.

A disadvantage for DSCs is their lower efficiency. The stability of these devices over time and temperature for various outdoor applications is not good. The organic solvent present in the electrolyte also causes problems such as evaporation or leakage of the electrolyte which reduces the device performance.

\subsubsection{Solid-state dye-sensitized solar cells}

The solid-state dye-sensitized solar cell (ssDSCs) was fabricated in order to avoid the problems of leakage/volatility of organic solvent in the electrolyte of DSCs. Initially it was tried with ionic liquid as electrolyte and then organic hole conductors such as either small organic molecular hole conductors (e.g. 2, 2', 7, 7'-tetrakis-( $N, N$-di-pmethoxyphenyl-amine) 9, 9'spiro-bifluorene (spiro-OMeTAD)) or conducting polymers (e.g. poly (ethylenedioxythiophene, (PEDOT)) were used. In organic hole conductors, the positive charge moves by a hopping mechanism between neighbouring molecules, whereas in redox couple (liquid), charge transfer happens due to movement of the redox species. There are trace amounts of additives also added to organic hole conductors to enhance some ionic conductivity [64]. The solid-state fabrication was done by incorporation of a solid-state hole transport material (HTM) such as spiro-OMeTAD, polythiophene, polyaniline, triphenylamine derivative N,N'-diphenyl-N,N'-bis(3- 
methylphenyl)-[1,1'-diphenyl]-4,4'-diamine (TPD), poly-3-hexylthiophene (P3HT) or polypyrrole into the nanomatrix of the dyed semiconductor photo-anode. This impregnation of the HTM was done either by spin coating or in situ photoelectrochemical deposition. In this case no electrolyte was used. We will discuss below details of the associated materials, principles and mechanism.

The photo-anode is prepared as the procedure discussed for liquid dye-sensitized solar cells. In ssDSC, a variety of dyes have been used as photo sensitizers especially ruthenium based organometallic dyes; namely N719, Z907, K-51, K68, P3-TAA-PHT, HRS-1 dye, and zinc-porphyrin dye. Very few metal-free organic dyes have been used for this kind of solar cell. Quantum dots, e.g. PbS, have also been used as photo-sensitizers for solid-state dye-sensitized solar cells. A variety of dyes used for solid-state dyesensitized solar cells in the literature are shown in Figures 1.7 and 1.8 [62].
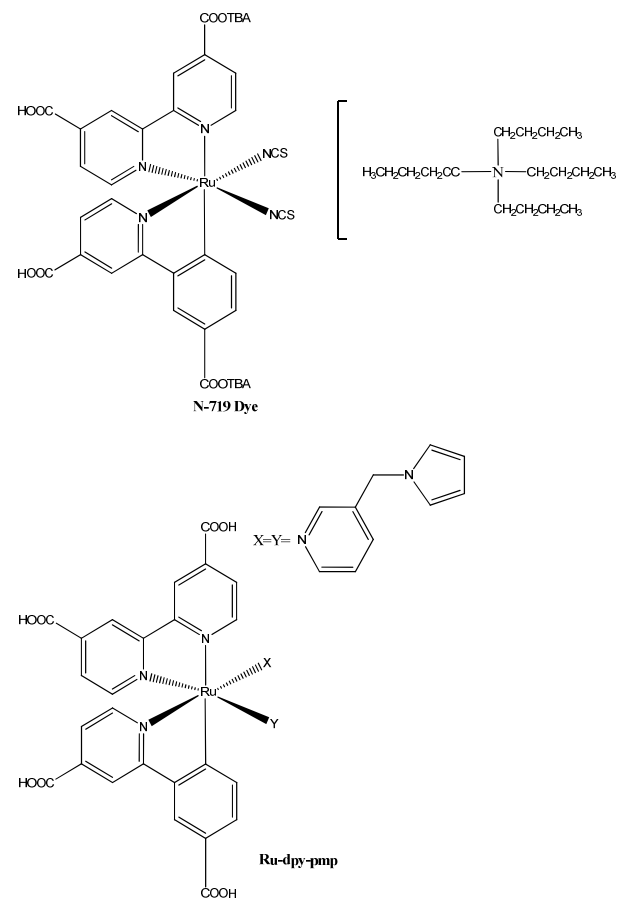

Figure 1.7: Photo-sensitizer molecules used for solid-state dye-sensitized solar cells. 

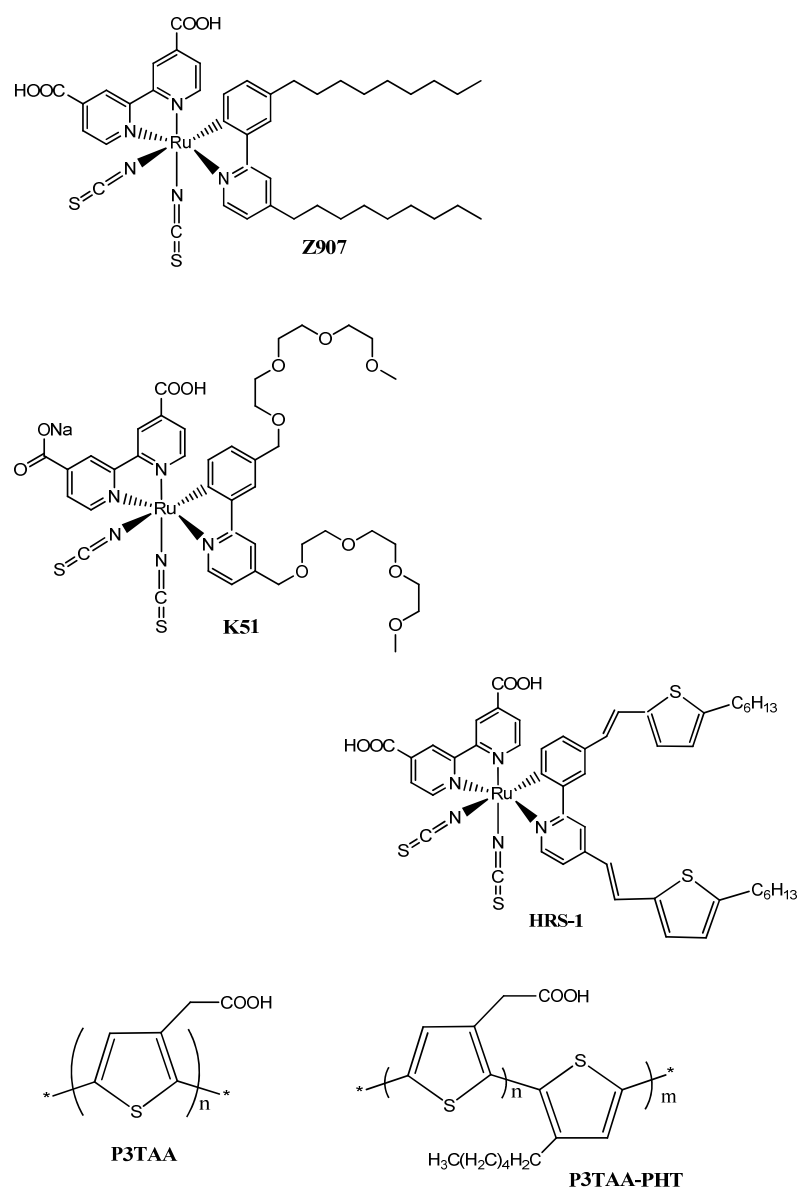

Figure 1.8: Other dye molecules used for ssDSCs.

Mostly gold/ platinum based electrodes are used as counter electrodes. The problem with a metal evaporator is that some of the gold particles diffuse into the photo-anode, which causes a short circuit and moreover there is degradation of organic materials under high temperature and vacuum during evaporation. During the last 10 years, extensive research has been done on this kind of hetero-hybrid inorganic and organic based solid-state solar cells. A performance of up to $6 \%$ efficiency has been achieved; which is the minimum requirement for starting up any commercial company or manufacturing unit. These kinds of devices are good when compared to their counterpart liquid based DSCs as far as stability are concerned. The real motivation for this type of DSC is the potential to fabricate such devices on a reel-to-reel basis on plastic or other flexible substrates. 


\subsubsection{Hole transporting material (HTM)}

Common hole transporting materials (HTMs) such as the p-type semiconductor (spiroOMeTAD) [88], inorganic based HTM (e.g. CuI, CuSCN) [89,89a] and polymer based conducting polymers (polythiophene and polypyrrole) [90-91] have been used. Various types of hole transport materials used to date for solid-state dye-sensitized solar cells are shown below in Figures 1.9 and 1.10 [62]. The selectivity of the hole transporting material and the method for its complete incorporation are the key challenges. Yanagida et al. reported in situ photo-electrochemical polymerization of a conducting polymer (polypyrrole) as a HTM for the fabrication of ssDSC but found it to be only $0.1 \%$ efficient [91]. Later Yanagida et al. tried with PEDOT as the HTM, which improved the overall power conversion efficiency up to $2.8 \%$ [92]. Yanagida et al. have tried with different dyes, different counter electrodes, different dopant anions and different HTMs for the successful photo-electrochemical polymerization to enhance the efficiency of ssDSCs. Recently, polyaniline has been used as a HTM but the efficiency was low (0.5\%). Poly (3-octylylthiophene), poly (3-hexylythiophene) [93-94] has been also used as HTMs in inorganic-organic solar cells. Poly (3-hexylythiophene) doped with ionic liquid and tertbutylypyridine increases the device performance up to $2.7 \%$ [95].

Triarylamine based compounds such as spiro-OMeTAD are the best HTMs among all organic hole conductors available to date for efficient (5.1\%) solid-state dye-sensitized solar cell application [96]. The problem with this HTM is that the rate of recombination is higher as compared to the redox couple used in liquid DSCs [97]. In this spiro-OMeTAD system, it is the poor conduction of the oxide film which limited the ssDSC performance [98]. The photo-current is directly proportional to the efficient pore filling by the HTM in the dyed $\mathrm{TiO}_{2}$ matrix, the ease of hole transfer, and the interface energetics rather than the conductivity of the HTM itself. A tris-[4-(2-methoxy-ethoxy)-phenyl]-amine (TMEPA) liquid based HTM gives the same performance with solid counterpart HTM. The poor performance of these ssDSCs is attributed the low pore filling of spiro-OMeTAD (only $60 \%$ ) with $3 \mu \mathrm{m}$ thickness of $\mathrm{TiO}_{2}$ into nanomatrix of dyed $\mathrm{TiO}_{2}$, which lowers the electronic contact between the dye molecules, $\mathrm{TiO}_{2}$ and the HTM [64]. 

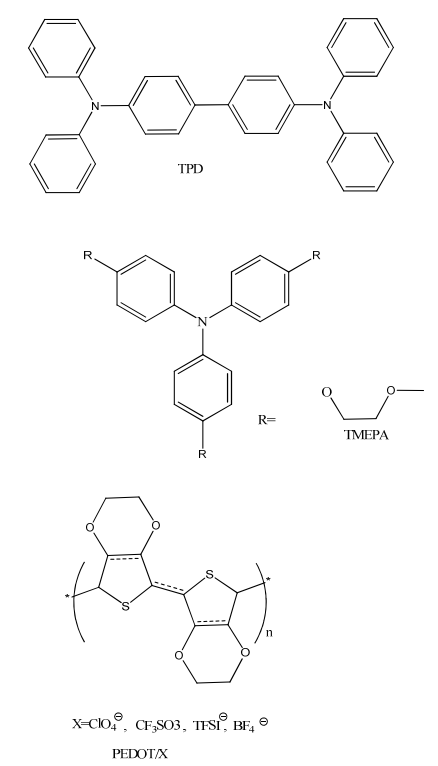

Figure 1.9: Various hole transporting materials (HTMs) used for solid-state DSCs.
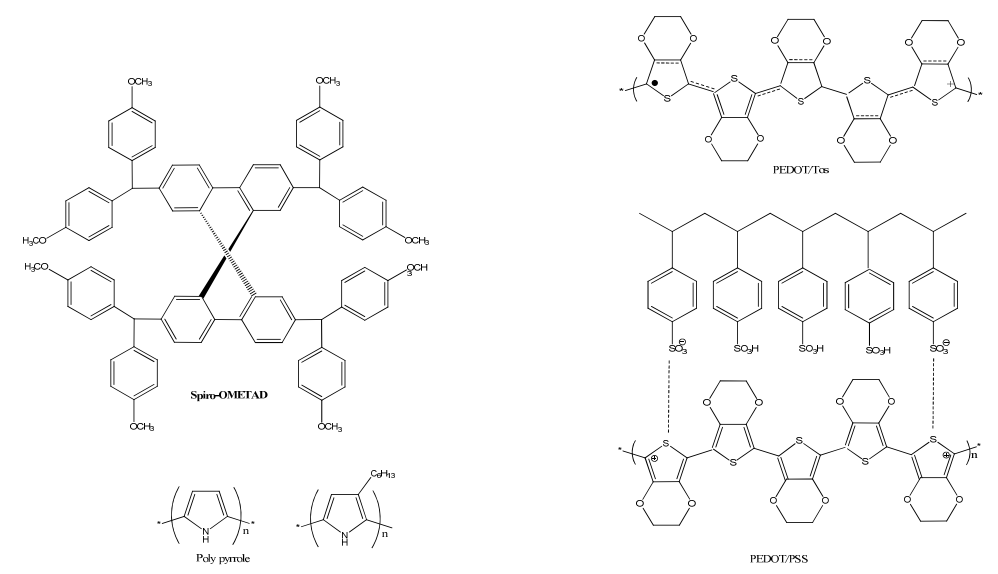

Figure 1.10: Other kinds of hole transporting materials (HTMs) used for ssDSCs.

\subsubsection{Operating principles and mechanism}

The HOMO level of the HTM must be above the HOMO level of the dye, and the LUMO level of the HTM must be lower than the conduction band of $\mathrm{TiO}_{2}$, in order to drive the 
charge transfer effectively. The HTM should be a transparent, conductive, amorphous, thin film to make the device efficient. The hole transporting material should be a transparent and amorphous thin film, otherwise crystallisation will hinder the pore filling which will lower photo-chemical, thermal stability hence reducing the carrier mobility. These HTMs must have a high glass transition temperature. Their work function should match well with the work function of the working electrode and counter electrode, and they should have good mobility. The operating principles and mechanism of solid-state DSC have been illustrated by Howie et al. [28] for a D-149 dye system. A schematic of the operating principles and structure of the spiro-OMeTAD based solid-state DSC are shown in Figures 1.11 and 1.12, respectively. Good features of spiro-OMeTAD are its work function of $4.9 \mathrm{eV}$, hole mobility of $10^{-4} \mathrm{~cm}^{2} \mathrm{~V}^{-1} \mathrm{~s}^{-1}$, amorphous structure, good solubility, organic non-crystalline nature and small molecule size, which make it a better hole transporting material within solid-state DSCs.

In solid-state DSCs, the dyed $\mathrm{TiO}_{2}$ acts as a light harvester and at the same time as an electron transporter. Charge separation takes place in between the dyed $\mathrm{TiO}_{2}$ and hole transporting material. The power energy conversion efficiency of the solid state DSC is mainly dependent on the charge transfer reaction at the heterojunction interface between dyed $\mathrm{TiO}_{2}$ and the hole transporting material. The electron transfer dynamics was first reported by Bach et al. [88] by picosecond transient absorption laser spectroscopy in 1999. He experimentally observed the phenomenon of photo induced electron, interfacial charge separation within the heterojunction of dyed $\mathrm{TiO}_{2}$ and HTM (spiro-OMeTAD) by this technique. The dye (Ru-based, N719) regeneration by HTM occurs in a picoseconds time domain with multiphase kinetics. This kinetics is one order of magnitude faster than with the usual redox couple $\left(\mathrm{I}^{-} / \mathrm{I}_{3}^{-}\right)$used in liquid dye-sensitized solar cells [99]. The rapid injection of hole from dye to HTM is a key challenge to fabricate efficient solid-state DSCs. Haque et al. [100] were the first to measure the yield of hole transfer from dyed $\mathrm{TiO}_{2}$ to HTM (for p-type organic semiconductor, TPD) by transient absorption spectroscopy, and found that this hole transfer is not only controlled by kinetic competition at the heterojunction interface $\mathrm{TiO}_{2} /$ dye/ HTM but also the $\Delta \mathrm{G}_{\text {dye-HTM}}$. This

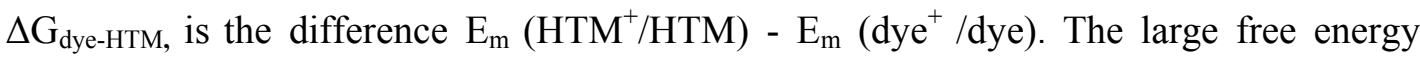
difference creates better local electrostatics at the interface, which is a key driving force 
for better hole transfer. The fast recombination reaction at the interface between $\mathrm{TiO}_{2}$ and HTM leads to the poor performance in ssDSC.

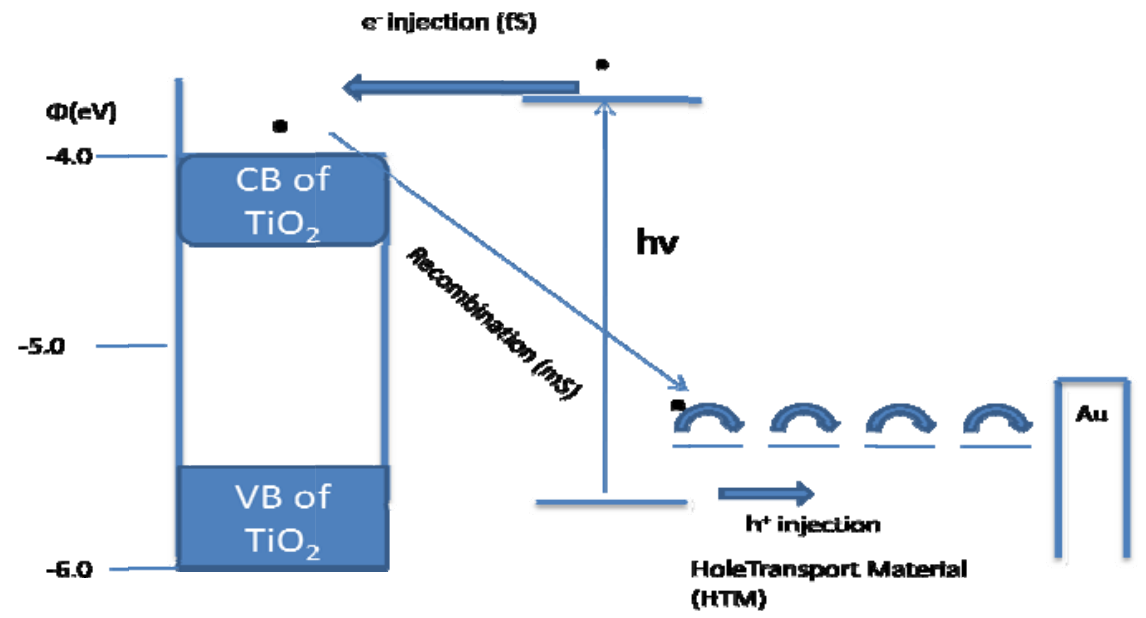

Figure 1.11: Operating principles of a spiro-OMeTAD based solid-state dye-sensitized solar cell.

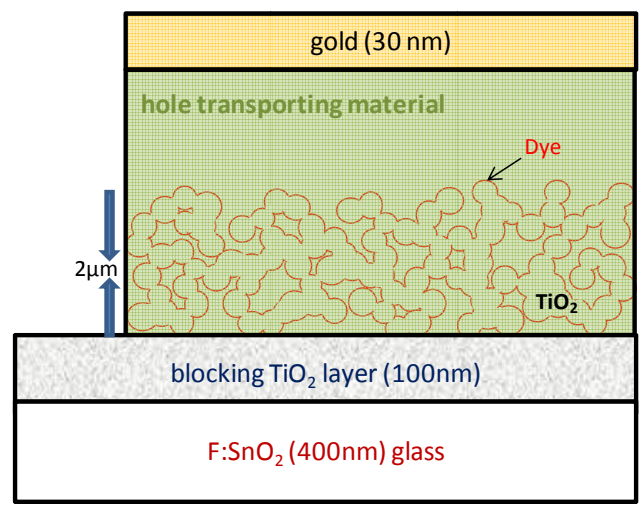

Figure 1.12: Schematic of a spiro-OMeTAD based ssDSC.

\subsection{Conducting polymers}

\subsubsection{Introduction to conducting polymers}

The discovery of conducting organic polymers in the mid-1970's has led to diverse applications in various fields of day to day life [101-103]. These novel organic polymers 
were previously believed to be insulators. The first discovery of semi-conductive polyacetylene received tremendous interest among material scientists. The conductivities of these organic materials were dependent on the process used in their synthesis. Furthermore, the conductivities could be enhanced when doped with different cations and anions such as $\mathrm{AsF}_{5}$, arsenic chloride, bromine, $\mathrm{ClO}_{4}^{-}$, iodine, $\mathrm{NOPF}_{6}$ or iron (ш) chloride. Although these conducting polymers are plastic, they conduct electricity with comparable capabilities as metals such as $\mathrm{Mg}$, In, $\mathrm{Sn}, \mathrm{Ge}$ when these are doped.

Various kinds of conducting polymers and their derivatives have been synthesised in the laboratory. This could be done by adding various side chains to the main backbone [104106]. These polymers are called electro-active polymers when they change their conductivities upon applying an external potential. The polymers change colour when switched between conductor, semiconductor and neutral states by changing the potential. Conducting polymers have become most fascinating because they show electronic, electrical and optical properties similar to a semiconductor or metal. Moreover, they exhibit adequate toughness, plasticity, resistance to friction, and elasticity properties [107-113].

The interesting variety of properties of conducting polymers led scientists to work in multidisciplinary fields. These research fields are associated with applications such as photovoltaics, electrochromics, transistors, diodes, sensors and charge storage (capacitor). Other areas of interest include organic light emitting diodes (OLED), biomedical applications, actuators, drug release, batteries, hydrogen storage, novel electrodes, molecular electronics, ion exchange membranes, antistatic coatings, packaging, printed circuit boards and smart textiles. These various applications rely on their conductivity and electro-activity [114-135]. The main drawbacks of these conducting polymers are limited processibility, solubility, and stability. There is extensive research going on by physicists, chemists, material scientists, electrical engineers and biologists to overcome these problems around the globe. The contribution of this exciting research was well recognised when the 2000 Nobel Prize in Chemistry went to three pioneer scientists, Prof. Allan Heeger (University of California, Santa Barbara, USA), the Late Prof. Allan MacDiarmid 
(University of Pennsylvania, USA), Prof. Hideki Shirakawa (University of Tsukuba, Japan).

\subsubsection{Thiophenes}

Shortly after the discovery of polyacetylene, the next conducting polymer polyparaphenylene was produced. The most popular and common conducting polymers are polypyrrole, polythiophene, polyaniline, and polyacetylene (Figure 1.13). The main early research focus of these materials was the investigation of their electrical conductivities.

Generally, conducting polymers in the neutral state behave as semiconductors or insulators. These can become conductive when an oxidising agent (electron acceptor) or reducing agent (electron donor) species is introduced; either by a chemical (by using reducing and oxidising agent) or electrochemical process (by applying potential). The level of doping determines the conductivity of the polymers concerned. The phenomena of p-doping and n-doping in conducting polymers are entirely different from that in inorganic based semiconductors. In inorganic semiconductors doping takes place when one or more atoms are replaced by atoms having more or less electrons. In the case of conducting polymers, this occurs because of an insertion or donation of electrons by insertion of dopant moieties. 


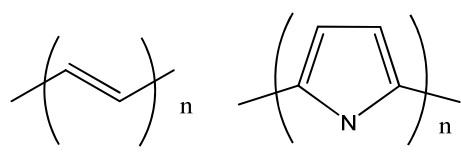

trans-Polyacetylene

Polypyrrole

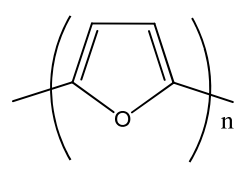

Polyfuran

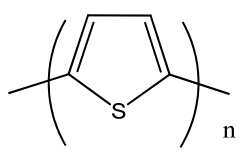

Polythiopene

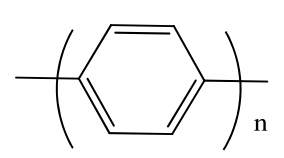

Polyparphenylene

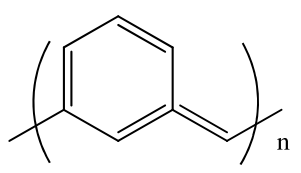

Polyheptadiyne

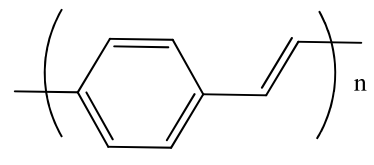

Polyparaphenylene vinylene

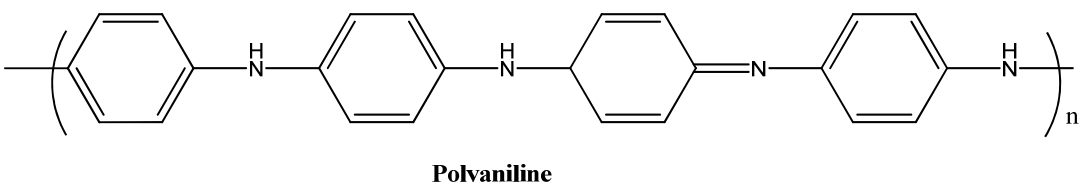

Figure 1.13: The most popular kinds of conductive polymers.

Polythiophene and its substituted derivatives have been well studied. They dominate various aspects of applications due to their exciting properties. The $\pi$-conjugation (alternating single and double bonds) of conducting polymers is delocalised over the entire backbone and the repeating monomer units, which makes them conductive. When a redox reaction occurs in an electrochemical system, i.e. n-doping (reduction) and pdoping (oxidation), it results in the insertion or removal of ionic species $\left(\mathrm{X}^{-}, \mathrm{A}^{+}\right)$(Figure $1.14)$.

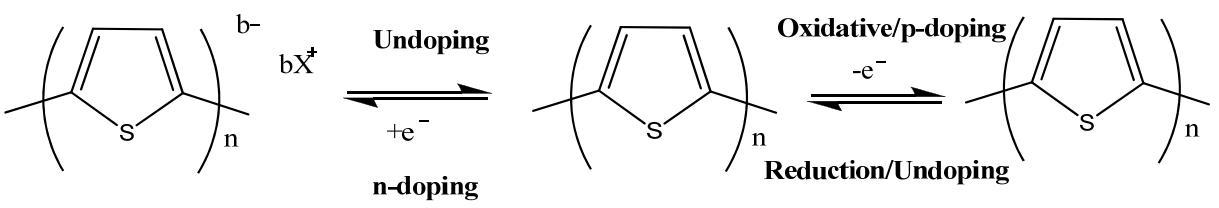

Doped/Conducting State Neutral/Semi-Conducting State

Doped/Conducting State

Figure 1.14: The n-doping and p-doping of polythiophene resulting in the neutral, semiconducting, or conducting state. 
Polymerization can be effected via two different processes, either electrochemically or chemically (by oxidant). Initially, the oxidation of the monomer leads to formation of a radical cation monomer that then combines with another cation to form a dimer. When this intermediate species loses two protons, a neutral state is achieved. Subsequent further oxidation and loss of protons produces trimers and so on until finally we get the polymer (shown in Figure 1.15).

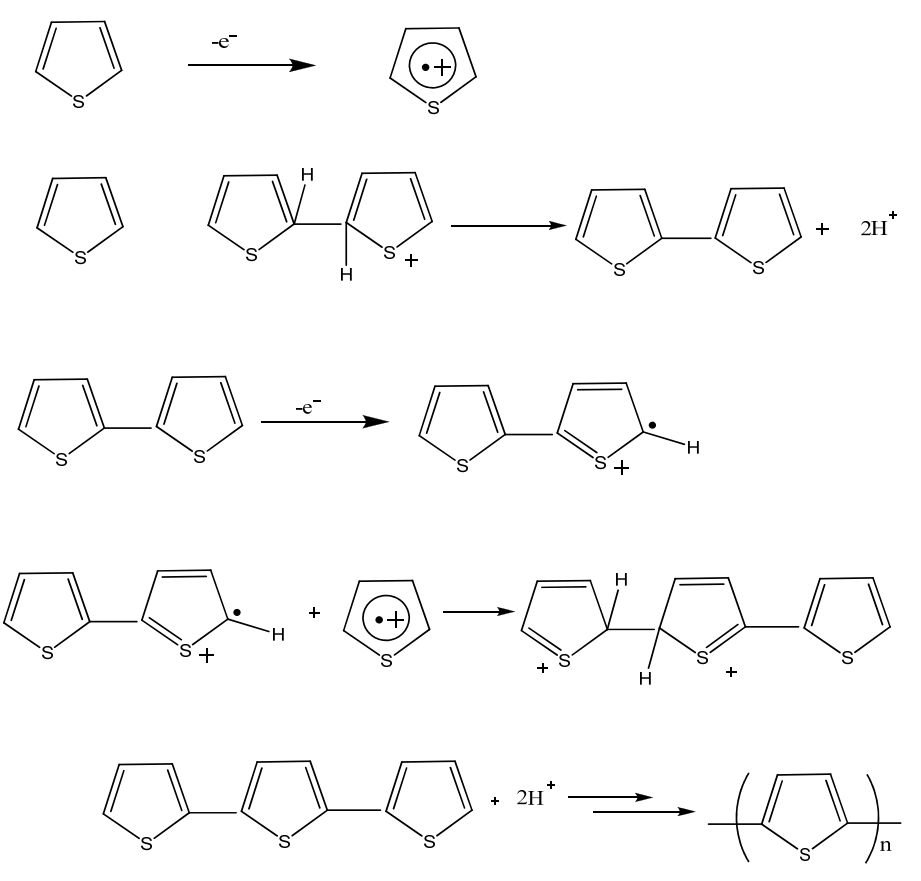

Figure 1.15: The proposed mechanism of polymerization of thiophene to polythiophene.

\subsubsection{Applications of PEDOT and its mechanisim in solar cells}

Among all polythiophene based conducting polymers, PEDOT has been used extensively used in different applications such as organic solar cells, organic light emitting diode (OLED), different conductive surface coating, actuator and sensor, antistatic and anticorrosion materials, artificial muscles, electrode materials in batteries, supercapacitors, display devices, and biosensors.and many more [ 136-138]. The application of PEDOT in solar cells is due to its transparency in the visible range, high hole conductivity $(550 \mathrm{~S} / \mathrm{cm})$, thermal stability, chemical and electrochemical stability. Other 
conducting polymers such as polypyrrole, polyaniline etc absobs light which restricts the light harvesting and at the end the efficiency of the photovoltaic devices decreases.

PEDOT is made of its monmer EDOT. There are various methods have been reported to prepare PEDOT such as electrochemical oxidation, chemical vapour polymerisaton, photoelectrochemical polymerisation, chemical methods. PEDOT is not soluble in many common solvents and moreover it is is not stable in its neutral state. It oxidises very fast under air /moisture contact [136].

The chemical stability and processability have been enhanced by adding polyelectrolyte such as polystyrene sulfonate (PSS) into it. The aqueous solution of PEDOT: PSS is highly conductive and PEDOT remains in its oxidised state. The work function of PEDOT is $5.2 \mathrm{eV}$ which makes it as a suitable hole transporting material for organic solar cells.The aqueous solution of PEDOT:PSS is extremely useful for the application of making thin, transparent and conductive surface coating on different surface.The morphology and conductivity of the film depends on the particle size, different level of doping concentration, solvent and method of preapration.The different methods employed to prepare PEDOT films on various conductive and non-condunctive substrates are electrochemical polymerization (const. potential and const. current growth), photoelectrochemical, dip coating, spin coating and chemical vapour polymerization techniques.The desired thickness of the films can be tailored by controlling various parameters such as concentration of monomer/oligomer, nature of the solvent, different electrolytes, time of PEDOT growth, potential, current, oxidants.

The various applications of energy harvesting and storage devices are as follows:

1. Organic light emmiting diode.

2. PEDOT based solid-state DSCs

3. Blocking layer used in bulk-hetrojunction solar cells

4. Used as counter electrode in Gratzels cells

5. Polymer based battery 
The different 15 resonance structures for the bis-EDOT radical cation are presented here. Therefore PEDOT structure is really well stabilised. Therefore, stabilisation of bigger oligomers can be well imagined from these resonant structures.

Resonance structures of the bis-EDOT radical cation
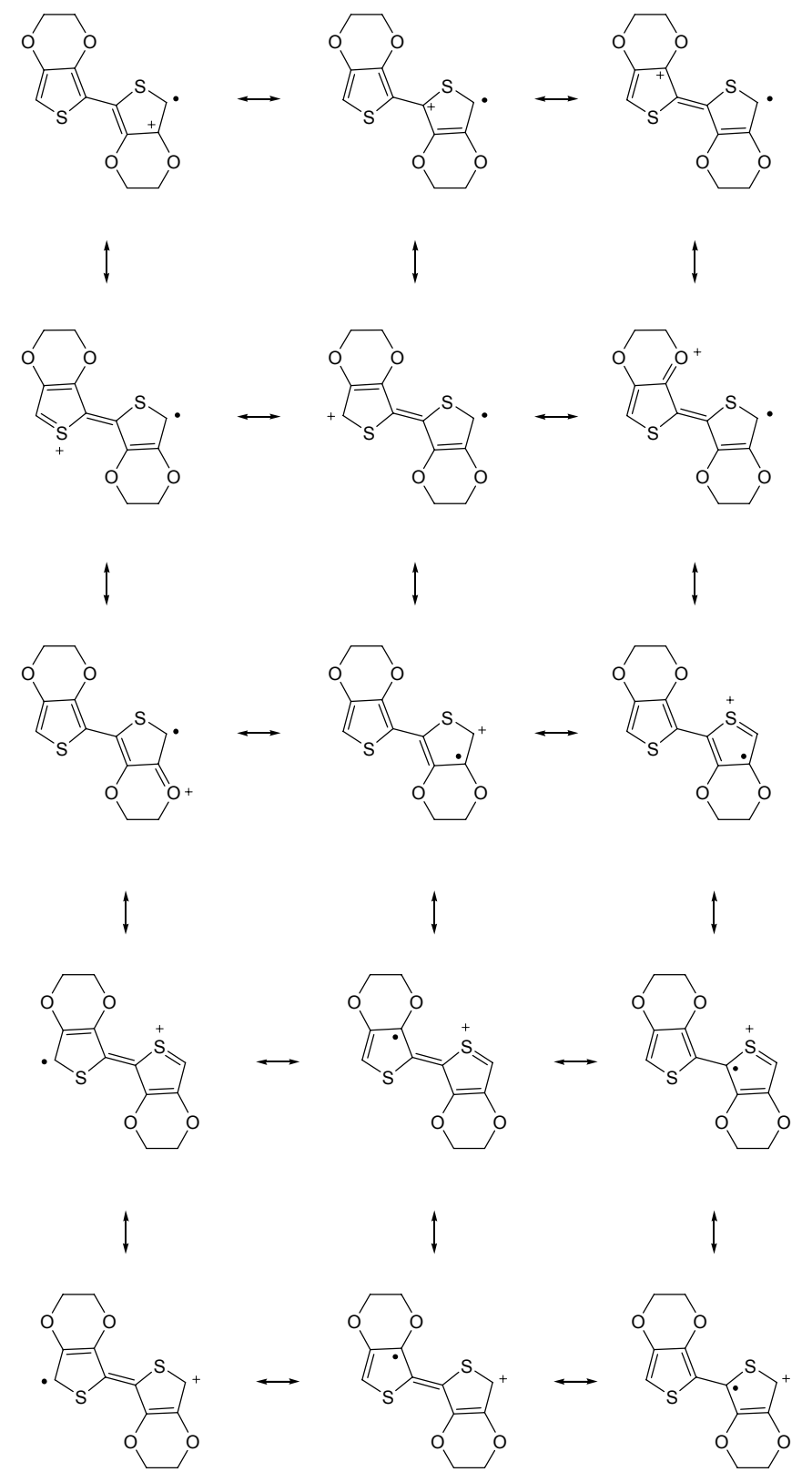

Figure1.15 a: Various resonance structure of bis-EDOT radical cation. 
The formation of discontinuities in conjugation (and therefore conductivity) in PEDOT formed by polymerization of bis-EDOT is presented here in Figure $1.15 \mathrm{~b}$. The radical cation (1) formed by addition of a bis-EDOT radical cation to a molecule of bis-EDOT will deprotonate to form the neutral, stabilised radical 2 (Figure 1.15b).

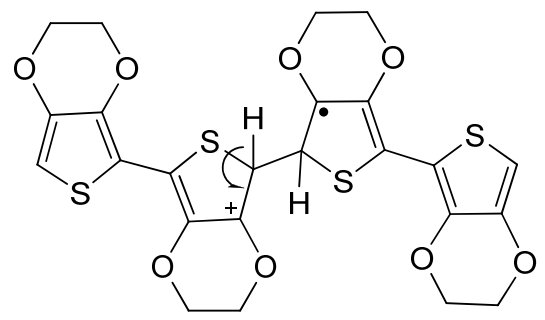

1

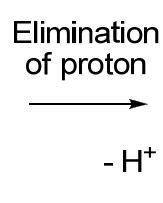

Figure: 1.15 b

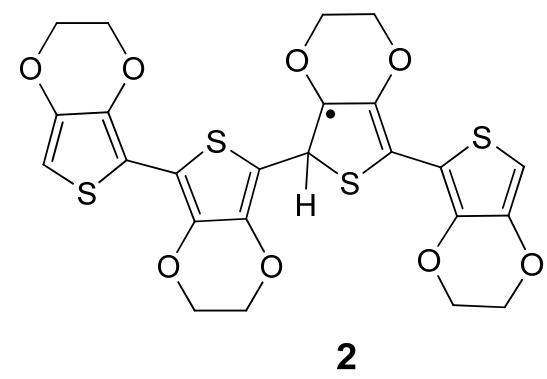

The reaction of the homologous, neutral, radical resonance structures like 2 with a molecule of bis-EDOT probably becomes increasingly favoured compared to one electron oxidation as the PEDOT chains get longer (and less soluble) and encounters with oxidants lessen in frequency. This means that as the PEDOT chains get longer, they are less mobile to come into an encounter with an oxidant, and thus the probability of reactions which lead to defects increases.

2. Even though the oxidation potentials for the PEDOT homologous series gets progressively smaller as the chains get longer, the oxidation potential for the related neutral free radical species such as $\mathbf{2}$ will probably be relatively invariant with chain length. The reason for this is that delocalisation of the unpaired electron is restricted to the final two EDOT units on the chain terminus because of interrupted conjugation. Thus, species which are easier to oxidise will react with the oxidant first, increasing the likelihood of reactions of the longer chains which lead to insulative defects.

The charge consumed during polymerization monitored by e-DAQ potentiostat and taken as a measure of the mass of the deposited PEDOT into photo-anode. The doping ion in the PEDOT always gives the level of electrical conductivity. PEDOT can be made 
conductive upon doping; when it oxidized it produces p-type doped materials where as upon reduction it gives n-type doped materials.Upon p-doping electrons are removed frpm conducting polymer leaving behind the positive charge (holes) in the $\pi$ - electron orbitals. The electrical conductivity can be achieved by when the holes can be filled up by $\pi$ - electrons hopping from the adjacent molecule.

The PEDOT used as a hole transporting material in solid-state DSC is presented and its principle shown in Figure 1.15c [139] where as its mechanism shown in Figure 1.15d. The manonolayer charge transfer dyed $\mathrm{TiO}_{2}$ film is placed in contact with PEDOT. Photo-excitation of dye injects electron into the conduction band of semiconductor $\mathrm{TiO}_{2}$. The dye regeneration takes place once the electron donated by PEDOT. The PEDOT can be regenerated at the counter electrode and the electrical circuit completes when electron migration takes place through external load. The perfect charge separation takes place when the PEDOT filled up in the pores of dyed $\mathrm{TiO}_{2}$ and the PEDOT should be well connected dyed $\mathrm{TiO}_{2}$. The performance of the solid-state DSCs depend on the pore filling of PEDOT into the nanomatrix dyed $\mathrm{TiO}_{2}$, morphology, dooping level of PEDOT and hence its conductivity and the electronic connectivity of PEDOT with dyed $\mathrm{TiO}_{2}$ film. [139].

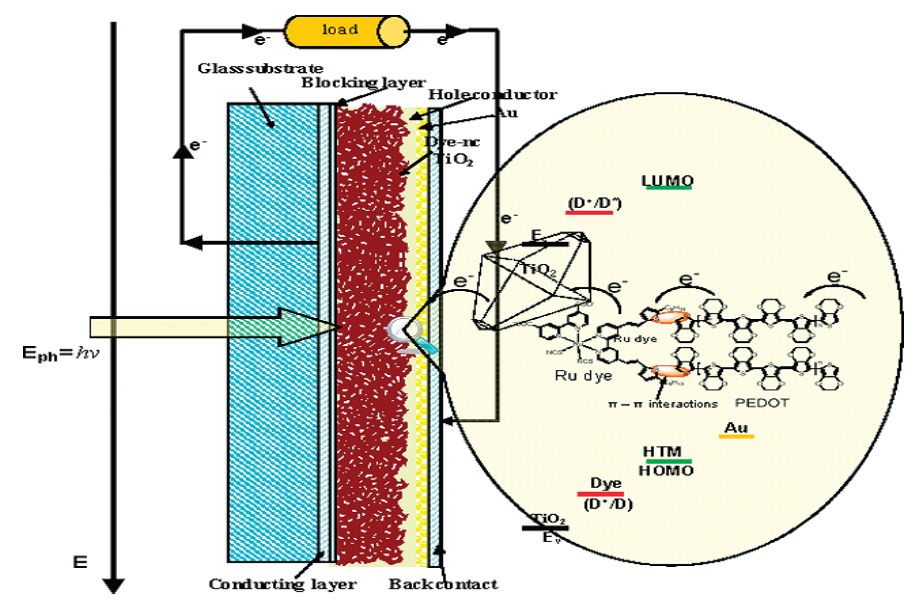

Figure: 1.15 c: Schematic of PEDOT based solid-state DSCs (Source: 42, 11, (2009) 1827-1838, Accounts of Chemical Research. 


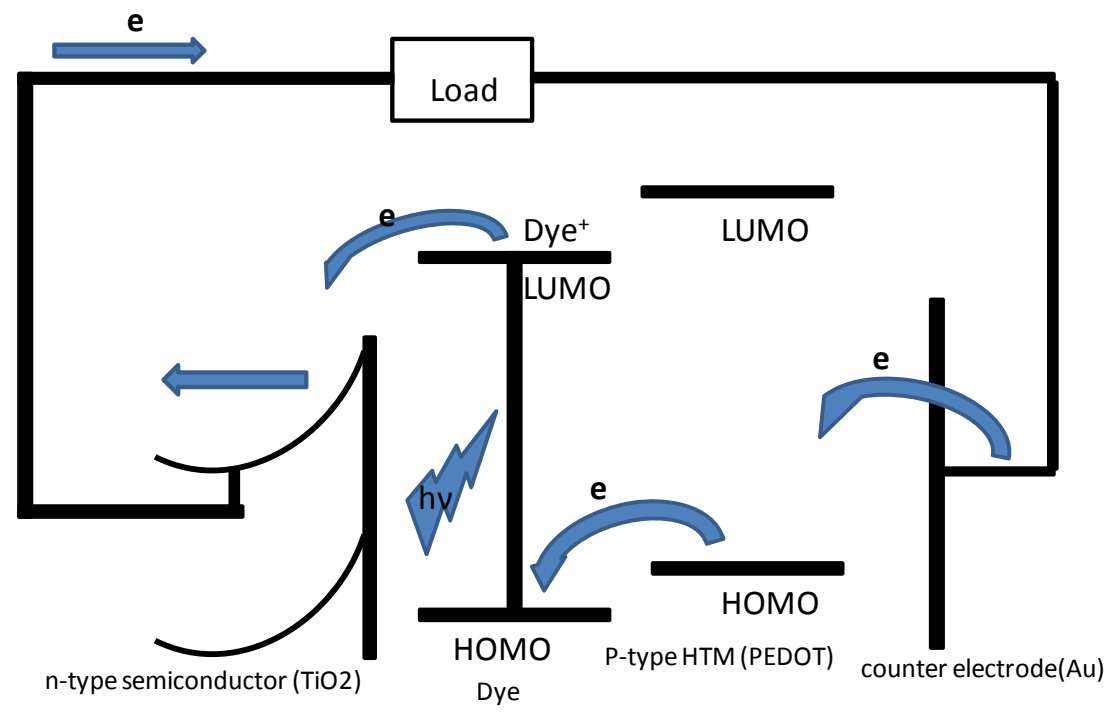

Figure 1.15 d: Operation and mechanisim of PEDOT based solid-state DSCs.

\section{4 General concept of a photovoltaic device}

The efficiency of each PV device is measured by its power conversion efficiency. The current voltage (JV) curve was used to extract crucial parameters for the device. These parameters are the fill factor $(\mathrm{FF})$, open-circuit voltage $\left(V_{\mathrm{oc}}\right)$, and short-circuit current density $\left(J_{\text {sc }}\right)$ which is obtained from the JV curve.

Short-circuit current $\left(J_{\text {sc }}\right)$ : This is the current generated by the solar cell, when the voltage is zero.

Open-circuit voltage $\left(V_{\mathrm{oc}}\right)$ : This is the voltage developed when the current is zero. In other words, it is the maximum voltage delivered by a solar cell when the current is zero.

Fill factor $(\mathrm{FF})$ : This is a measure of the squareness of the JV curve. This value depends on the ratio of two areas shown in Figure 1.16. This ratio will be unity under ideal conditions i.e. when there are no parasitic resistance losses in the devices. Resistance has a great impact on PV devices. Shunt resistance and series resistance are important for the 
fill factor. In ideal conditions, series resistance should be low and shunt resistance should be high in the cell.

So $\mathrm{FF}=(\operatorname{Vmp} \times \operatorname{Imp}) /\left(V_{\text {oc }} \times J_{\text {sc }}\right)$

Where $\mathbf{V m p}=$ voltage at maximum power point, $\mathbf{I m p}=$ current at maximum power point.

Efficiency ( $\boldsymbol{\eta}$ ): This is the ratio of the electrical power from the PV device to the total incident light power $\left(\mathrm{P}_{\text {in }}\right)$.

$$
\begin{aligned}
\boldsymbol{\eta}_{\text {AM 1.5 }} & =\mathbf{P}_{\text {out }} / \mathbf{P}_{\text {in }} \\
& =\left(J_{\text {sc }} \times V_{\text {oc }} \times \mathbf{F F}\right) / \mathbf{P}_{\text {in }}
\end{aligned}
$$

Where $\mathrm{P}_{\text {out }}=$ electrical power generated by the cell at maximum power point (mpp), and $\mathrm{P}_{\text {in }}=$ Incident light power.

Maximum power point (mpp): It is the point where the product of current and voltage reaches a maximum. In addition, any device must be operated at this point. $I_{m p p}$ and $V_{m p p}$ are the characteristic voltage and current at this particular point.

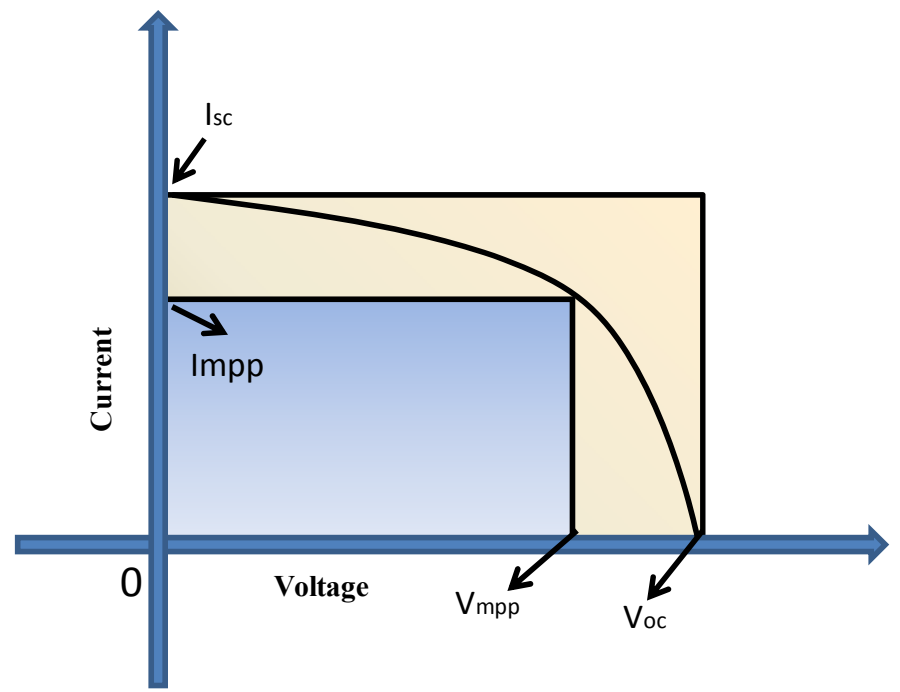

Figure 1.16: Illustration of important photovoltaic parameters of any type of solar cell.

Equivalent circuit of solar cells: An equivalent circuit (Figure 1.17) of the PV device gives a clear picture about the current source, voltage source, series resistance and shunt resistance. The current source represents the photocurrent generated when the device is 
illuminated. The nature of the flowing current is just the opposite to a diode and is dependent on the potential across the device. A series resistance originates from the ohmic contribution from the different electrodes, independent resistivity power of the associated materials or contact between semiconductor and metal. A lesser resistance aids the achieving of maximum efficiency. This series resistance increases with an increase in light intensity and temperature, whereas it decreases with thickness of the electrode. The shunt resistance affects the loss of current within the device; the higher the shunt resistance, the better is the device performance. This resistance decreases dramatically when we increase the light intensity, whereas the resistance increases with decrease of the thickness of the electrode. The circuit elements in Figure 1.17 are defined as, $\mathrm{R}_{\mathrm{s}}=$ series resistance, $\mathrm{R}_{\mathrm{SH}}=$ shunt resistance, $\mathrm{I}_{\mathrm{PH}}=$ photo-current $\mathrm{U}=$ applied voltage.

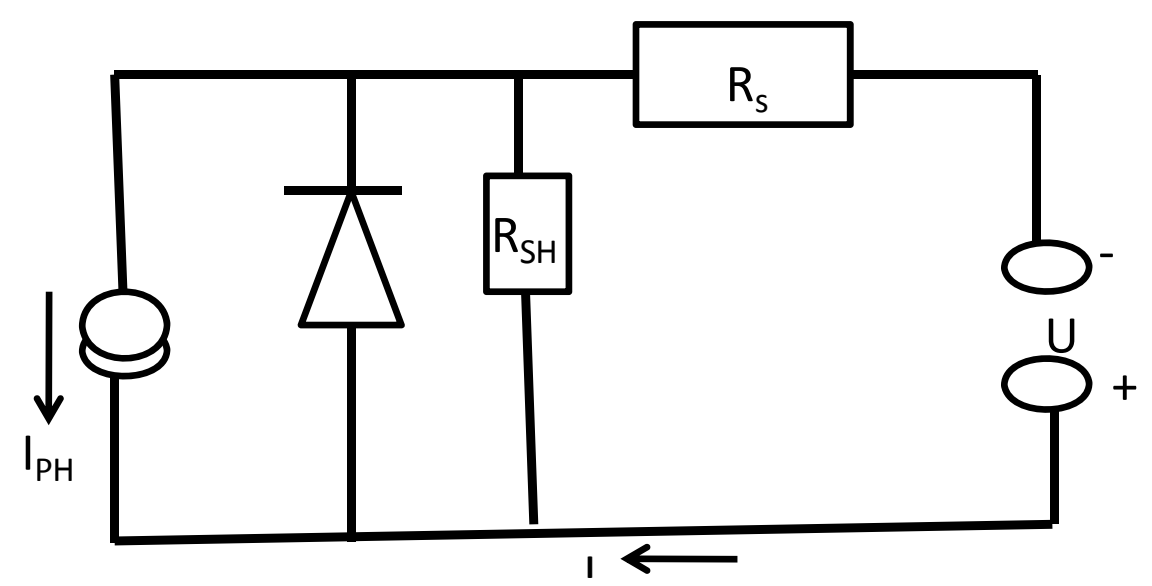

Figure 1.17: Equivalent circuit of single junction solar cells

\subsection{Thesis aims and structure}

The main aims of my thesis are:

- A novel in situ photo-electrochemical polymerization (PEP) will be employed for the fabrication of solid-state dye-sensitized solar cells (ssDSCs).

- To evaluate the suitability of synthesized porphyrin dyes, and commercially available ruthenium and indoline, and organic dyes for the in situ photoelectrochemical deposition of PEDOT for solid state solar cells. 
- To establish a reliable procedure for rapid screening of suitable photosensitizing dyes / monomers for photo-electrochemical deposition.

- To use conducting polymer PEDOT as hole transporting material (HTM) in ssDSCs.

- To fabricate efficient dye-sensitised solar cells, both solid-state and liquid.

- To fabricate flexible solid-state dye-sensitised solar cells.

- Optimisation of photovoltaic performance of solid-state dye-sensitized solar cells.

- To test and compare the photovoltaic performance of both solid-state and liquid dye-sensitized solar cells.

- Characterisation of these device as well as working electrode will be done by various techniques such as Raman spectroscopy, UV-Visible-near Infrared (UVVis-NIR) spectroscopy, matrix assisted laser desorption ionisation (MALDI) and mass spectroscopy.

- Atomic Force Microscopy (AFM), SEM (scanning electron microscopy) and SEM-EDXA (SEM-Energy Dispersive X-ray Analyser) will be used to establish the morphology of the PEDOT/dyed $\mathrm{TiO}_{2}$ working electrode (photo anode).

The structure of this thesis is as follows:

(i) This overall theme is the fabrication and characterisation of novel nanostructured solid-state dye sensitized solar cells. Component materials, photovoltaic performance, ease of fabrication and light weight were considered. The advantages and disadvantages of the novel cells were compared with standard Grätzel cells.

(ii) Chapter 2 describes the materials, methods and experiments that were employed in the thesis.

(ii) Chapter 3, the state-of-art of fabrication of PEDOT based solid-state dye-sensitized solar cells is achieved by the standard photo-electrochemical deposition method. A photosensitizer dye attached to a large band gap inorganic semiconductor $\left(\mathrm{TiO}_{2}\right)$ serves as the primary light harvesting moiety, and an electro-active conducting polymer as the hole conducting medium. This is done to reproduce established literature results. This chapter also discusses different hole transporting material and different condition employed for the fabrication of solid-state dye-sensitized solar cells. 
iii) Chapter 4 presents the morphological determination of photo-anode grown by a photo-electrochemical method. This includes the growth of PEDOT, its morphology, and mechanism in an efficient solid-state dye-sensitiszed solar cell. The enhancement of the photovoltaic performance of this PEDOT based solid-state DSCs was well studied by various powerful instrumental characterisation tools such as UV-Vis-NIR, Raman, SEM, SEM-EDXA, AFM, and Transient Absorption Spectroscopy. The main drawbacks of these kinds of solid-state dye-sensitized solar cells are the unoptimised filling and distribution of the conjugated polymer inside the pores of the nanostructured electrode. Better pore filling and photovoltaic performance are influenced by the polymerization conditions, such as illumination side, light intensity and film thickness. The fabrication of solid-state DSCs is demonstrated using nanocrystalline metal oxide $\mathrm{TiO}_{2}$ as an electron acceptor, ruthenium complex Z907 dye as a photo-sensitizer, PEDOT as hole transporting material (HTM), various dopants, solvents, a newly developed counter electrodes and different light intensities during photo-electrochemical polymerization.

(iv) Chapter 5 explores various types of counter electrodes (both metallic and nonmetallic). The three new materials that fulfil the criteria for counter electrodes for solidstate DSCs are: 1. conjugated polymers, graphene-based electrodes, and a novel compressible and flexible Goretex®-Au-PEDOT. The PV performances of these three counter electrodes are described. Gold based counter electrodes represent the most common counter electrode material used for solid-state dye-sensitized solar cells using photo-electrochemically deposited PEDOT hole conductor. The main problem with this gold/ ITO counter electrodes in our laboratory is lack of reproducibility. These issues are therefore addressed in this chapter.

(v) Chapter 6 explores the possibility of using different low cost metal free organic dyes, along with various zinc-porphyrin dyes as potential photo-sensitizers. The purpose of using a variety of dyes is to optimize the photo-polymerization process by combining them with novel designed photo-sensitizer/ conjugated polymers that will favour efficient charge transfer to increase the power conversion efficiency.

vi) Chapter 7 describes the general conclusions. 


\subsection{References}

1. Li, B., Wang, L., Kang, B., Wang, P., and Qiu, Y., Solar Energy Materials and Solar Cells, 90 (2006) 549-573.

2. Kamat, P.V., Journal of Physical chemistry C, 111 (2007) 2834-2860.

3. Li, C., Liu, M., Pschirer, N.G., Baumgarten, M., and Mu"llen, K., Chemical Review, 110 (2010) 6817-6855.

4. http://www.stanford.edu/group/mcgehee/presentations/McGehee2009.pdf

5. Dillon, A. C., Chemical Review, 110 (2010) 6856-6872.

6. Solar buzz retail module index. www.solarbuzz.com.

7. G.L.Pearson, 18th IEEE photovoltaic specialist's conference, PV founder's award luncheon (1985).

8. Chapin, D.M., Fuller, C.S., and Pearson, G.L., Journal of Applied Physics, 25 (1954) 674.

9. Saga, T., NPG Asia Mater, 2 (2010) 96-102.

10. Photon International, 176, March 2009.

11. Sirimanne, P.M., and Perera, V.P.S., Physica Status Solidi (b), 245 (2008) $1828-1833$.

12. Petrozza, A., Groves, C., and Snaith, H.J., Journal of the American Chemical Society, 130 (2008) 12912-12920.

13. Grätzel, M., Inorganic Chemistry, 44 (2005) 6841-6851.

14. Kroeze, J.E., Hirata, N., Schmidt-Mende, L., Orizu C., Ogier S.D., Carr, K., Grätzel, M., and Durrant J.R., Advanced Functional Materials, 16 (2006) 18321838.

15. Snaith, H.J., Moule, A.J., Klein, C., Meerholz, K., Friend, R.H., and Grätzel, M., Nano Letters, 7 (2007) 3372-3376.

16. Snaith, H.J., Humphry-Baker, R., Chen, P., Cesar, Ilkay, Zakeeruddin, S.M., and Grätzel, M., Nanotechnology, 19 (2008) 424003 (12 pp).

17. Snaith, H.J.and Grätzel, M., Physical Review Letters, 98 (2007) 177402-4.

18. Bisquert, J., Cahen, D., Hodes, G., Ruhle, S., and Zaban A., Journal of Physical Chemistry B, 108 (2004) 8106-8118.

19. Kamat P.V., Journal of Physical Chemistry C, 112 (2008) 18737-18753. 
20. Liang, M., Xu, W., Cai, F., Chen, P., Peng, B., Chen, J., and Li, Z., Journal of Physical Chemistry C, 111 (2007) 4465-4472.

21. Snaith, H.J., and Schmidt-Mende, L., Advanced Materials, 19 (2007) 31873200 .

22. Campbell, W.M., Burrell, A.K., Officer, D.L., Jolley, K.W., Coordination Chemistry Reviews, 248 (2004) 1363-1379.

23. Peter, L.M., Journal of Physical Chemistry C, 111 (2007) 6601-6612.

24. Hodes, G., Journal of Physical Chemistry C, 112 (2008) 17778-17787.

25. Gao, F., Wang, Y., Shi, D., Zhang, J., Wang, M., Jing, X., Humphry-Baker, R., Wang, P., Zakeeruddin, S.M., and Grätzel, M., Journal of the American Chemical Society, 30 (2008) 10720-10728.

26. Biancardo, M., West, K., and Krebs, F.C., Journal of Photochemistry and Photobiology A: Chemistry, 187 (2007) 395-401.

27. Snaith, H.J., Whiting, G.L., Sun, B., Greenham, N.C., Huck, W.T.S., and Friend, R.H., Nano Letters, 5, (2005) 1653-1657.

28. Howie, W.H., Claeyssens, F., Miura, H., and Peter, L.M., Journal of the American Chemical Society, 130 (2008) 1367-1375.

29. Lowman, G.M., and Hammond, P. T., Small, 1 (2005) 1070 -1073.

30. Eder, D., and Windle, A.H., Advanced Materials, 20 (2008) 1787-1793.

31. Honda, K., Journal of Photochemistry and Photobiology A: Chemistry, 166 (2004) 63-68

32. Nozik, A.J., Annual Physical Chemistry Reviews, 29 (1978) 189-222.

33. Honda, K., and Fujishima, A., Nature, 238 (1972) 37-39.

34. Bard, A.J., Science, 207 (1980) 139-144.

35. O'Reagen, B., and Grätzel, M., Nature, 353 (1991) 737-740.

36. Kay, A., and Grätzel, M., Journal of Physical Chemistry, 97 (1993) 6272-6277.

37. Suliman, A. E., Tang, Y. W., and Xu, L., Solar Energy Material and Solar Cells, 91 (2007) 1658.

38. Furube, A., Murai, M., Watanabe, S., Hara, K., Katoh, R., and Tachiya, M.., Journal of Photochemistry and Photobiology A, 182 (2006) 273.

39. Sayama, K., Sugihara H., and Arakawa, H., Chemistry Material, 10 (1998) 3825 . 
40. Wang, Q., Ito, S., Grätzel, M., Fabregat-Santiago, F., Mora-Sero, I., Journal of Physical Chemistry B, 110 (2006) 25210-25221.

41. Kalansundaram, K., and Grätzel, M., Coordination Chemistry Reviews, 77 (1998) 347-414.

42. Palomares, E., Clifford, J.N., Haque, S.A., Lutz, T., and Durrant, J.R., Journal of the American Chemical Society, 125 (2003) 475-482.

43. Tennakone, K., Perera, V.P.S., Kottegoda, I.R.M., and Kumara, G., Journal of Physics D-Applied Physics, 32 (1999) 374.

44. Taguchi, T., Zhang, X.T., Sutanto, I., Tokuhiro, K., Rao, T.N., Watanabe, H., Nakamori, T., Uragami, M., and Fujishima A., Chemical Communication, 19 (2003) 2480.

45. Kumara, G.R.A., Okuya, M., Murakami, K., Kaneko, S., Jayaweera V.V., and Tennakone, K., Journal of Photochemical Photobiology A: Chemistry, 164 (2004) 183

46. Huynh, W., Peng, X., and Alivisatos, A.P., Advanced Materials, 11 (1999) 923927.

47. Snaith, H.J., and Schmidt-Mende, L., Advanced Materials, 19 (2007) 31873200 .

48. Boucle, J., Ravirajan, P., and Nelson, J., Journal of Material Chemistry, 17 (2007) 3141-3153.

49. Gur, IIan, Fromer, N.A., Chen, C.-Ping., Kanaras, A.G., and Alivisatos, A.P, Nano Letters, 7 (2007) 409-414.

50. Mozer, A.J., Wada, Y., Jiang, K., Masaki, N., Yanagida, S., and Mori, S.N., Applied Physics Letters, 89 (2006) 043509.

51. Hagfeldt, A., and Grätzel, M., Chemical Reviews, 95 (1995) 49-68.

52. Tang, C.W., Applied Physics Letters, 48 (1986) 183.

53. Gunes, S., Neugebauer, H., and Sariciftci, N.S., Chemical Reviews, 107 (2007) 1324-1338.

54. Gui, L., Hao, L. G., Niu, Y.X., and EnLe, Z., Chinese Science Bulletin, 52 (2007) 145-158.

55. Li, C., Chen, Y., Wang, Y., Iqbal Z., Chhowalla, M., and Mitra, S., Journal of Material Chemistry, 17 (2007) 2406-2411. 
56. Schmidt-Mende, L., Campbell, W. M., Wang, Q., Jolley, K.W., Officer, D.L., Nazeeruddin, M. K., Grätzel, M., ChemPhysChem, 6 (2005) 1253-1258.

57. Wienk, M.M., Kroon, J.M., Verhees, W.J.H., Knol, J., Hummelen, J.C., van Hal P.A., Janssen, R.A.J., Angewandte Chemie International Edition, 42 (2003) 3371-3375.

58. Kim, J.Y., Lee, K., Coates, N.E., Moses, D., Nguyen, T.-Q., Dante, M., and Heeger, A.J., Science, 317 (2007) 222-225.

59. Triyana, K., Yasuda, T., Fujita, K. and Tsutsui, T., Thin Solid Films, 477 (2005) 198-202.

60. Berger, O., Inns, D., and Aberle, A.G., Solar Energy Materials and Solar Cells, 91 (2007) 1215-1221.

61. Sanchez, C., Julian, B., Belleville, P., and Popall, M., Journal of Material Chemistry, 15 (2005) 3559-3592.

62. Yanagida S., Yu, Y., and Manseki, K., Accounts of Chemical Research, 42 (2009) 1827-1838.

63. Howie, W.H., Claeyssens, F., Miura, H., and Peter, L. M., Journal of the American Chemical Society, 130 (2008) 1367-1375.

64. Hagfeldt, A., Boschloo, G., Sun, L., Kloo, L., and Pettersson, H., Chemical Review, 110 (2010) 6595-6663.

65. Hamann, T. W., Jensen, R. A., Martinson, A. B. F., Van Ryswyk, H., and Hupp, J. T., Energy Environment Science, 1 (2008) 66.

66. Pagliaro, M., Palmisano, G., Ciriminna, R., Loddo, V., Energy Environment Science, 2 (2009) 838

67. Jose, R., Thavasi, V., and Ramakrishna, S., Journal of American Ceramic Society, 92 (2009) 289.

68. Zhang, Q. F., Dandeneau, C. S., Zhou, X. Y., and Cao, G. Z., Advanced Material, 21 (2009) 4087.

69. Hwang, S., Lee, J.H., Park, C., Lee, H., Kim, C., Park, C., Lee, H., Lee, W., Park, J., Kim, K., Park, N-G., and Kim, C., Chemical Communications, 46 (2007) 4887-4889.

70. Kuang, D., Klein, C., Snaith, H. J., Moser, J-E, Humphry-Baker, R., Comte, P., Zakeeruddin, S.M., and Grätzel, M., Nano Letters., 6 (2006) 769-773 
71. Nazeeruddin, M. K., Humphry-Baker, R., Liska, P., and Grätzel, M., Journal of Physical Chemistry B., 107 (2003) 8981-8987.

72. Mozer, A.J., Wagner, P., Officer, D.L., Wallace, G. G., Campbell, W.M., Miyashita, M.,, Sunahara, K., and Mori, S., Chemical Communications, 39 (2008) 4741-4742.

73. Mozer, A.J., Griffith, M.J., Tsekouras, G., Wagner, P., Wallace, G. G., Mori, S., Sunahara, K., Miyashita, M., Earles, J. C., Gordon, K.C., Du, L., Katoh, R., Furube, A. and Officer, D.L., Journal of the American Chemical Society, 131 (2009) 15621-15623.

74. Howie, W.H., Claeyssens, F., Miura H., and Peter, L.M., Journal of the American Chemical Society, 130 (2008) 1367-1375.

75. Kitamura, T., Ikeda, M., Shigaki, K., Inoue, T., Anderson N.A., Ai, Xin., Lian, T., and Yanagida, S., Chemistry Materials, 16 (2004) 1806-1812.

76. Rawling, T., Austin, C., Buchholz, F., Colbran, S.B. and McDonagh, A.M. Inorganic Chemistry, 48 (2009) 3215-3227.

77. Wang, Z-S., Cui, Y., Dan-oh Y., Kasada, C., Shinpo A., and Hara, K., Journal of Physical Chemistry C, 111 (2007) 7224-7230.

78. Tian, H., Yang, X., Cong, J., Chen, R., Teng, C., Liu, J., Hao, Y., Wang, L., and Sun, L., Dyes and Pigments, 84 (2010) 62-68.

79. Hagberg, D.P, Yum, J-H., Yum, Lee, H.J., De Angelis F., Marinado, T., Karlsson, K. M, Humphry-Baker, R, Sun, L., Hagfeldt, A., Grätzel, M., and Nazeeruddin, M.K., Journal of the American Chemical Society, 130 (2008) 6259-6266.

80. Teng, C., Yang, X., Yuan, C., Li C., Chen, R., Tian, H., Li, S., Hagfeldt, A., and Sun, L., Organic Letters, 11 (2009) 5542-5545.

81. Zafer, C., Kus, M., Turkmen, G., Dincalp, H., Demic, S., Kuban, B., Teoman, Y., and Icli, S., Solar Energy Materials and Solar Cells, 91 (2007) 427-431.

82. Alex, S., Santosh, U., and Das, S, Journal of Photochemistry and photobiology A: Chemistry, 172 (2005) 63-71.

83. Denaro, T., Bagilo, V., Girolamo, M., Antonucci, V., Arico, A, S., Matteucci, F., and Ornelas, R., Journal of Applied Electrochemistry, 39 (2009) 2173-2179. 
84. Imoto, K., Takahashi, K., Yamaguchi, T., Komura, T., Nakamura. J., and Murata, K., Solar Energy Materials and Solar Cells, 79 (2003) 459-469.

85. Suzuki, K., Yamaguchi, M., Kumagai, M., and Yanagida, S., Chemistry Letters, 32 (2003) 28-29.

86. Roy-Mayhew, J., Bozym, D.J., Punckt C., and Aksay, I.A., ACS Nano, 4 (2010) 6203-6211.

87. Ahmad, S., Yum J-H, Butt, H-J., Nazeeruddin, M.K., and Grätzel Michael, Chemical Physics and Physical Chemistry, 11 (2010) 2814-2819.

88. Bach, U., Lupo, D., Comte, P., Moser, J. E., Weissörtel, F., Salbeck J., Spreitzer, H., and Grätzel, M., Nature, 395 (1998) 583-585.

89. Tennakone, K., Kumara, G., Kumarasinghe, A. R., Wijayantha, K. G. U., Sirimanne, P. M., Semiconductor Science Technology, 10 (1995) 1689-1693.

89a. O'Regan, B., Lenzmann, F., Muis, R., and Wienke, J., Chemistry Material, 14 (2002) 5023-5029.

90. Saito, Y., Kitamura, T., Wada, Y., and Yanagida, S., Chemistry Letters, 31 (2002) 1060-1061.

91. Murakoshi, A.K., Kogure, R., Wada, Y., and S. Yanagida, Chemistry Letters, 5 (1997) 471-472.

92. Xia, J., Masaki, N., Lira-Cantu, M., Kim, Y., Jiang, K., and Yanagida S., Journal of the American Chemical Society, 130 (2008) 1258-1263.

93. Zhang, W., Cheng, Y., Yin, X., and Liu, B., Macromolecular Chemistry and Physics, 212 (2011) 15-23.

94. Günes S., Neugebauer, H., and Sariciftci, N.S., Manuscript for ECS Transactions "Fullerenes, Nanotubes, and Carbon Nanostructures", The Electrochemical Society, 2 (2007) 145-154

95. Jiang, K. J., Manseki, K., Yu, Y. H., Masaki, N., Suzuki, K., Song, Y. L., and Yanagida, S., Advanced Functional Material, 19 (2009) 2481.

96. Snaith, H. J., Moule, A. J., Klein, C., Meerholz, K., Friend, R. H., and Grätzel, M., Nano Letters, 7 (2007) 3372-3376.

97. Bach, U., Tachibana, Y, Moser, J-E., Haque, S.A., Durrant, J.R., Grätzel, M., and Klug, D.R., Journal of the American Chemical society, 121 (1999) 74457446. 
98. Yum, J.H., Chen, P., Grätzel, M., and Nazeeruddin, M.K., ChemSusChem, 1 (2008) $699-707$.

99. Li, B., Wang, L., Kang, B., Wang, P., and Qiu, Y., Solar Energy Materials \& Solar Cells, 90 (2006) 549-573.

100. Haque, S.A., Park, T., Holmes, A.B., and Durrant, J.R., Chemical Physics and Physical Chemistry, 4 (2003) 89-93.

101. Heeger, A.J., Reviews of Modern Physics, 73 (2001) 681-700.

102. Shirakawa, H., Synthetic Metals, 125 (2001) 3-10.

103. MacDiarmid, A.G., Synthetic Metals, 125 (2001) 11-22.

104. Chiang, C.K., Fincher, J.C.R., Park, Y.W., Heeger, A.J., Shirakawa, H., and Louis, E. J., Physical Review Letters, 39, (1977) 1098.

105. Chiang, C.K., Gau, S.C., Fincher, J.C.R.., Park, Y.W., and MacDiarmid, A.G., Applied Physics Letters, 33 (1978) 18.

106. Chiang, L.Y., Garito, A.F., and Sandman, D.J., Electrical, Optical, and Magnetic Properties of Organic Solid-State Materials, Material Research Society Symposium Proceedings No. 247 (Materials Research Society, Pittsburgh) (1992).

107. Skotheim, T.A., Elsenbaumer, R.L., and Reynolds, J.R., Handbook of Conducting Polymers, 2nd ed., (Marcel Dekker, New York), p 85.

108. MacDiarmid, A.G., and Epstein, A.J., 1990, Conjugated Polymeric Materials: Opportunities in Electronics, Optical Electronics and Molecular Electronics, edited by Bredasandand, J.L., and Chance, R.R., (Kluwer Academic, Dordrecht), p.53.

109. Wallace, G.G, Spinks, G.M, Kane-Maguire, L.A.P, and Teasdale, P.R., Conductive Electroactive Polymers: Intelligent Polymer Systems, Third Edition, CRC Press (2008).

110. Maniloff, E.S., Vacar, D., McBranch, D.W., Wang, H., Mattes, B.R., Gao, J., and Heeger, A.J., Optics Communications, 141 (1997) 243-246.

111. McMillan, W. L., Physical Review B, 24 (1981) 2739.

112. McGehee, M.D., and Heeger, A.J., Advanced Materials, 12 (2000) 1655-1668.

113. McGehee, M.D., Miller E. K., Moses, D., and Heeger, A.J., In Advances In Synthetic Metals: Twenty Years of Progress in Science and Technology, edited 
by P. Bernier, S. Lefrant, and G. Bidan (Elsevier Science, Lausanne), p. 98.(1999).

114. Mozer, A.J., Panda, D.K., Gambhir, S., Romeo, T. C., Winther-Jensen, B., and Wallace, G.G., Langmuir, 26 (2010) 1452-1455.

115. Nielsen, C.B., Angerhofer, A., Abboud, K.A., Reynolds, J.R., Journal of the American Chemical Society, 130 (2008) 9734-9745.

116. Beaujuge, P., Ellinger, S., Reynolds, J.R., Nature Materials, 7 (2008) 795-799.

117. Yang, Y., Farley, R.T., Steckler, T.T., Eom, S.-H., Reynolds, J.R., Schanze, K. S., and Xue, J., Applied Physics Letters, 93 (2008) 163305.

118. Zhang, X., Steckler, T.T., Raghunath, D.R., Ohira, S., Potscavage, W.J., Tiwari, S., Ellinger, S., Barlow, S., Bredas, J.-L., Kippelen B., Reynolds, J.R., and Marder, S. R., Journal Material Chemistry, 20 (2010) 123-134.

119. Amb, C.M., Beaujuge, P.M., and Reynolds, J.R., Advanced Materials, 22 (2010) 724-728.

120. Wang, J.Z., Zheng, Z.H., Li, H.W., Huck, W.T.S. \& Sirringhaus, H., Nature Materials, 3 (2004) 171-176.

121. Tsumura, A., Fuchigami, H., and Koezuka, H., Synthetic Metals, 41 (1991) 1181-1184.

122. Gustafsson, G., Cao, Y., Treacy, G.M., Klavetter, F., Colaneri, N., and Heeger, A. J., Nature, 357 (1992) 477-479.

123. Janata, J., and Josowicz, M., Nature Materials, 2 (2003) 19-24.

124. Liu, Y., Cui T., and Varahramyan, K., Solid State Electronics, 47 (2003) 811814.

125. Gross, M., Müller, D.C., Nothofer, H.-G., Scherf, U., Neher, D., Bräuchle, C., and Meerholz, K., Nature, 405 (2000) 661-665.

126. Rossi, D.D., Santa, A.D., and Mazzoldi, A., Synthetic Metals, 90 (1997) 93-100.

127. Pernaut, J.-M., Journal of Physical Chemistry B, 104 (2000) 4080-4090.

128. Guimard, N.K., Gomez, N., and Schmidt, C.E., Progress in Polymer Science, 32 (2007) 876-921.

129. Chen, J, Liu, Y., Minett, A.I., Lynam, C., Wang, J., and Wallace, G.G., Chemistry Materials, 19 (2007) 3595-359. 
130. Winther-Jensen, B., Winther-Jensen, O., Forsyth, M., and MacFarlane, D.R., Science, 321 (2008) 671.

131. Winther-Jensen, B., Fraser, K., Ong, C., Forsyth, M., and MacFarlane, D.R., Advanced Materials, 22 (2010) 1727-1730.

132. Jurczyk, M.U., Kumar, A., Srinivasan, S., and Stefanakos, E., International Journal of Hydrogen Energy, 328 (2007) 1010-1015.

133. Green, R.A, Baek, S., Poole-Warren, L.A., and Martens, P.J., Science and Technology Advanced Materials, 11 (2010) 014107.

134. Akkerman, H.B., Blom P.W.M., Leeuw, D.M.de and Boer, B.D., Nature, 441 (2006) 69-72.

135. Kreuer, K.D., Journal of Membrane Science, 185 (2001) 29-39.

136. Zhou, C., Liu, Z., Yan, Yushan Du., X, Mai, Y-W., and Ringer S., Nanoscale Research Letters, 6:364, 2011.

137. Wei, D., and Amaratunga G., Int. J. Electrochem. Sci., 2 (2007) 897 - 912.

138. Huang, Y et.al, Pure Appl. Chem., 80, 11 (2008)2 241-2258.

139. Yanagida, S., Yu, Y., and Manseki, K., Accounts of Chemical Research, 42 (2009) 1827-1838. 


\section{EXPERIMENTAL METHODS}




\section{1 Chemical reagents and materials}

Chemicals used in this work were:

\section{Commercial}

Lithium perchlorate (Aldrich), acetonitrile (Aldrich), anhydrous tetrahydrofuran (THF, Aldrich), anhydrous ethanol (Aldrich), titanium isopropoxide (Wako, Japan), titanium diisopropoxide bis-acetylacetonate (TAA, Aldrich), tert-butylpyridine (Aldrich), lithium bis-trifluoromethanesulfonamide (LiTFSI, Aldrich), conc. nitric acid (Aldrich $70 \%$ ), chenodeoxylic acid (Fluka), ferrocene (Aldrich), 3-methoxy propionitrile (Aldrich), $\mathrm{TiO}_{2} \mathrm{~T}$-paste (Solaronix), Ti nanooxide 300 (Solaronix), Z907 dye (Solaronix), metal free D-149 dye supplied by CSIRO (Melbourne), 2,5dihydroxybenzoic acid, (DHB Aldrich), tetrabutylammonium hexaflurophosphate (Aldrich), tetrabutylammonium tetrafluroborate (Aldrich), chenodeoxylic acid (Aldrich), lithium trifluoromethanesulfonic acid $\left(\mathrm{LiCF}_{3} \mathrm{SO}_{3}\right.$, Aldrich), tetrabutylammonium hexafluoride phosphate (Aldrich), tetrabutyl ammonium hexafluoride borate (Aldrich), tetrabutylammonium perchlorate (Fluka), 1,2dimethyl-3-propylimidazolium iodide (DMPII, Solaronix), lithium iodide (LiI, Aldrich), and iodine ( $\mathrm{I}_{2}$, Aldrich)

"In-house synthesised"

bis-EDOT (bis-ethylenedioxythiophene) and tris-EDOT (synthesised in our lab) were synthesised by our collaborator Sanjeev Gambhir, ANFF and zinc porphyrin dyes (GD2, P-10 (dimer)) and P-257 dye were synthesised by our collaborator Pawel Wagner, IPRI. EMITFSI was synthesised by Sayed Ashraf (1-ethyl-3methylimidazolium bis-trifluoromethanesulfonamide (synthesised in our lab).

Other materials used were:

Fluorine doped tin oxide (FTO) coated glass (Nippon, Japan) having: (1) high conductivity (resistance $\sim 10 \Omega / \square$, transmission $\sim 75 \%$ ) and used for counter electrode; (2) high transmission (resistance $\sim 14 \Omega / \square$, transmission $\sim 80 \%$ ) that was 
used for working electrode. Indium doped tin oxide (ITO) coated glass (10 $\Omega / \square$, Delta Technologies, USA); and platinum mesh (Goodfellow, Cambridge, UK) (counter electrode).

\section{2 General instrumental techniques}

All electrochemical and photo-electrochemical polymerization experiments were carried out using an eDAQ electrochemical system. This electrochemical system comprises an e-recorder 401 with eDAQ and eChem software interfaced to a computer. This electrochemical apparatus was employed for recording cyclic voltammograms, potentiostatic growth, electrochemical polymerization, photoelectrochemical polymerization, and constant current growth. A schematic of the photo-electrochemical polymerization set-up is shown in Figure 2.1.

UV-Vis spectra of different dye solutions, oligomer solutions and photo-anode films were recorded using either a Shimadzu UV-1601 spectrophotometer or a Shimadzu UV-3600, UV-Vis-NIR spectrophotometer).

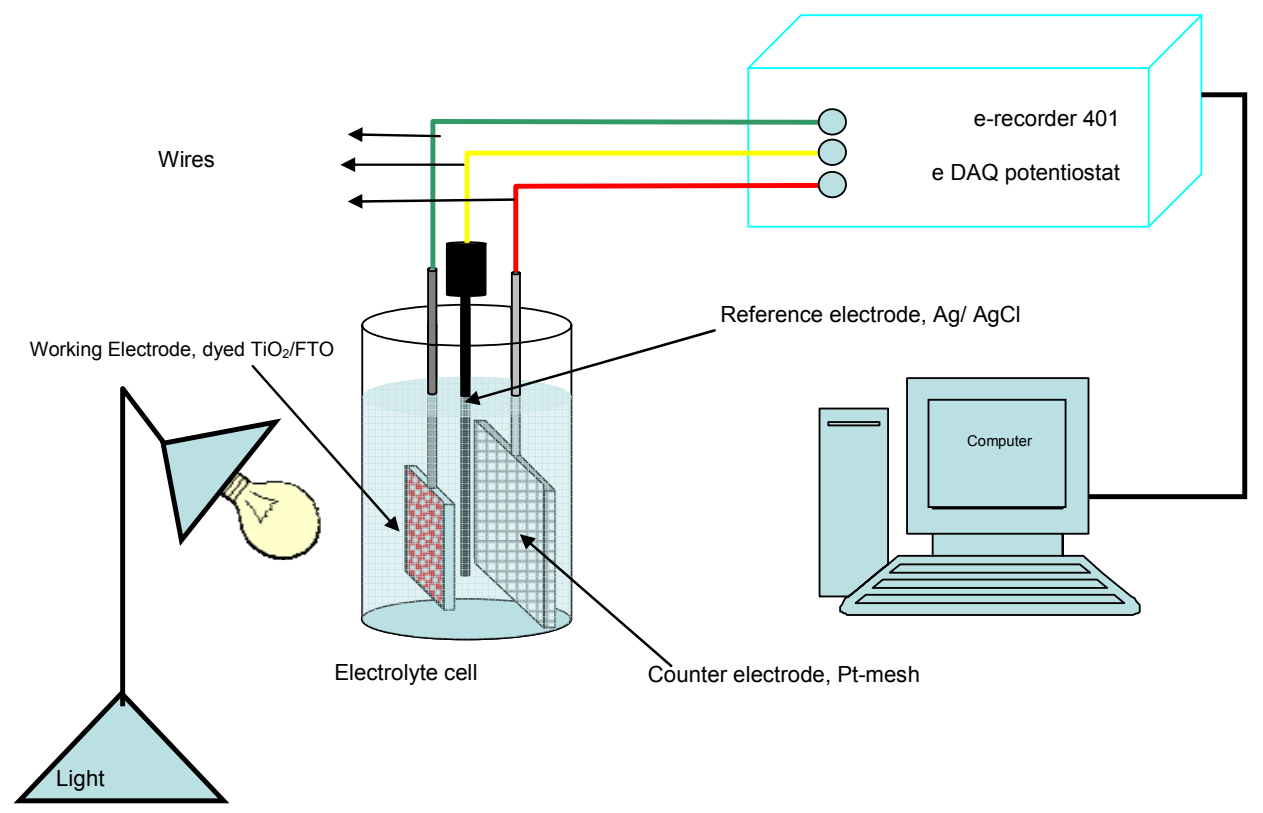

Figure 2.1: Schematic of photo-electrochemical polymerization set-up for the preparation of photo-anodes. 
The morphology of photo-anodes were characterized by both atomic force microscopy (Asylum Research, Atomic Force Microscope, MFP 3D), and scanning electron microscopy (SEM) and SEM-Energy Dispersive X-ray Analyser (JEOL, JSM -7500 FA). Raman spectroscopy was employed to study the various doping levels/ processes and oxidation states of PEDOT prepared by the photoelectrochemical method. Quantification of PEDOT deposited on photo-anodes (working electrode) over time was measured by Raman spectroscopy using a Jobin Yvon Horiba HR800 Raman spectrometer with Labspec software. Matrix assisted laser desorption ionisation (MALDI) MS were obtained using a Shimadzu Axima Confidence. MALDI samples were prepared with the matrix DHB (2, 5dihydroxybenzoic acid) using the solventless MALDI technique.

\subsubsection{Electrochemical polymerization}

Three kinds of electrochemical polymerization methods were used to grow the conducting polymer film on a conducting substrate, namely (i) constant current (galavanostatic growth), (ii) constant potential (potentiostatic growth) and (iii) cyclic voltammetry (CV) (potentiodynamic growth). Each experimental method was used to measure different parameters. The onset oxidation and reduction potentials of dyes, monomers, and oligomers could be calculated by cyclic voltammetry by measuring the current as a function of applied voltage. The first forward scan is where oxidation takes place at the working electrode. Reduction takes place in the reverse scan. Total charge consumed during polymerization was calculated by integrating the area under the CV curve.

This CV method was carried out in electrochemical cells comprised of 3 electrodes. The working electrode was ITO-coated glass, with platinum mesh as the counter electrode. The reference electrode was either $\mathrm{Ag} / \mathrm{Ag}^{+}$(if in organic media; silver wire dipped into solution containing $0.01 \mathrm{M} \mathrm{Ag} / \mathrm{NO}_{3}$ and $0.10 \mathrm{M}$ tetrabutylammonium perchlorate in acetonitrile), or $\mathrm{Ag} / \mathrm{AgCl}$ (if in aqueous media). Photo-electrochemical polymerization was done using a lower concentration of 
monomer or oligomer in solution compared to the supporting electrolyte such as 0.10 $\mathrm{M} \mathrm{LiClO}_{4}$ (10 times higher concentration).

The electrochemical polymerization method was used to prepare conducting polymer-based counter electrodes on various conducting substrates. These electrodes were prepared potentiostatically via the polymerization of bis-EDOT at $0.8 \mathrm{~V}$ vs. $\mathrm{Ag} / \mathrm{AgCl}$ at room temperature. The thickness of the PEDOT film was controlled by varying the growth time.

\subsubsection{Photo-electrochemical polymerization}

Photo-electrochemical polymerizations were carried out at constant potential using an apparatus shown in Figure 2.2. PEDOT was grown over time onto a nano-matrix of $\mathrm{TiO}_{2}$ containing dye. The dyed $\mathrm{TiO}_{2}$ substrate was immersed in a solution of 0.1 $\mathrm{M} \mathrm{LiClO}_{4}$ and $0.01 \mathrm{M}$ bis-EDOT in $\mathrm{CH}_{3} \mathrm{CN}$. The working electrode was illuminated from the mesoporous $\mathrm{TiO}_{2}$ side by a SoLUX lamp (12 V, $50 \mathrm{~W}$, and WaKO, Japan). The light intensity was varied using optical density neutral filters. The dye-adsorbed $\mathrm{TiO}_{2}$ substrate was used as the working electrode, $\mathrm{Pt}$ mesh as counter electrode, and $\mathrm{Ag} / \mathrm{AgCl}$ as the reference electrode. A constant potential of $0.2 \mathrm{~V}$ vs. $\mathrm{Ag} / \mathrm{AgCl}$ was applied at the working electrode (dyed $\mathrm{TiO}_{2}$ ). The current was monitored using eDAQ software.

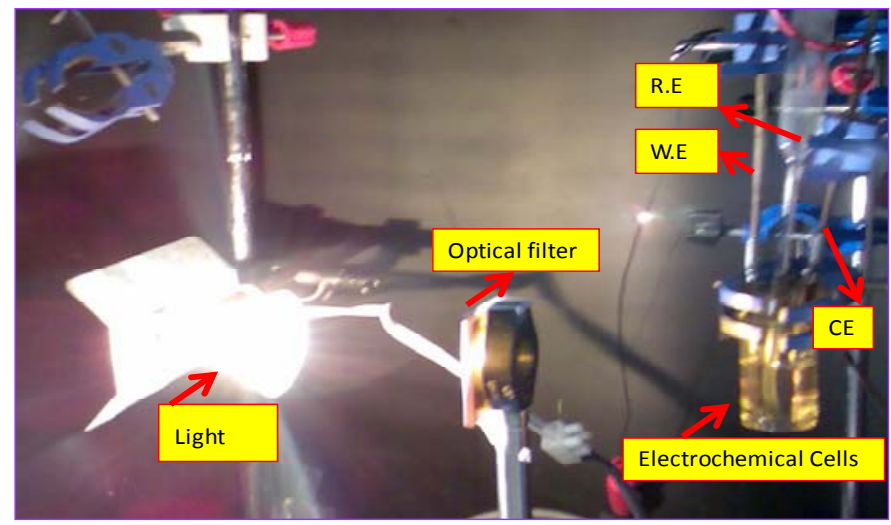

Figure 2.2: Apparatus used for the photo-electrochemical polymerization of bisEDOT on dyed $\mathrm{TiO}_{2}$. 


\section{3 Device structure of solid-state dye-sensitized solar cells}

Figure 2.3 shows the structure of the solid-state dye sensitized solar cells with each layer of the device presented in detail.

The following fabrication steps for the solid-state DSCs were employed for the fabrication of the device:

i. Cleaning of FTO substrates

ii. Compact layer of $\mathrm{TiO}_{2}$

iii. Prepare mesoporous $\mathrm{TiO}_{2}$ film layer by doctor-blading.

iv. Dye sensitisation of the $\mathrm{TiO}_{2}$ film.

v. Impregnation of hole transporting material in dyed- $\mathrm{TiO}_{2}$.

vi. Ionic liquid treatment on photo-anode

vii. Preparation of counter electrode.

viii. Device fabrication and testing

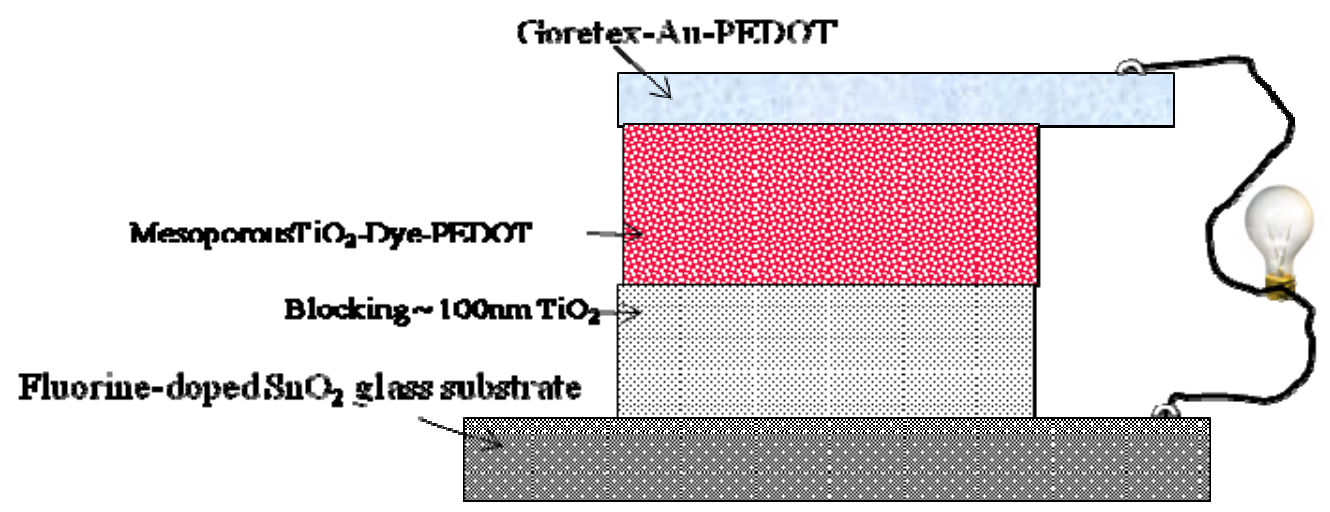

Figure 2.3: Structure of a PEDOT based solid-state dye-sensitized solar cell. 


\subsubsection{Cleaning of FTO substrate}

\subsubsection{Snow cleaning}

Foreign materials such as hydrocarbons need to be removed from the FTO substrate in order to enable maximum adhesion of coatings. $\mathrm{CO}_{2}$ "snow cleaning" was used to prepare FTO and other surfaces. In snow cleaning, very small dry ice particles are blasted at a surface at high velocity with the aid of compressed $\mathrm{CO}_{2}$ gas. At the surface, the dry ice particles can both abrade and dissolve impurities. The apparatus consists of a gun with a fine aperture connected to $\mathrm{CO}_{2}$ through PTFE (polytetrafluoroethylene) tubing. On blowing, expansion led to the creation of very minute dry ice particles with high streamlined gas velocity.

This technique was used to clean FTO glass substrates, used for both counter and working electrodes. The FTO glass was cut into $7 \mathrm{~cm} \times 3 \mathrm{~cm}$ rectangles using a diamond cutter. Before cutting, the conductivity of the FTO glass was checked, and then marked on the non-conducting side. Care was taken to avoid touching or scratching the conductive surface. The cut pieces were then held in place by vacuum and subjected to snow cleaning, spraying with $\mathrm{CO}_{2}$ uniformly. After cleaning, the vacuum was released and the sides handled only with plastic tweezers. After that, all cleaned FTO glass substrates were kept for UV ozone cleaning.

\subsubsection{UV-Ozone cleaning}

UV-Ozone cleaning was performed using a Jelight R model 42-220. Exposure to UV light and ozone removed final traces of any organic contaminants on the substrates. This cleaning was done for 18 minutes. 


\subsubsection{Compact/blocking layer of $\mathrm{TiO}_{2}$}

A compact/blocking $\mathrm{TiO}_{2}$ layer was deposited on an optically transparent conducting FTO glass (FTO, F: $\mathrm{SnO}_{2}$ ) by either spin coating or spray pyrolysis. The purpose of this blocking layer was to avoid an electrical contact between the hole transporting material (HTM) and the FTO which can cause a short-circuit of the solar cells.

\subsubsection{Spin coating}

Spin coating is an effective way of depositing a thin, uniform layer on a flat substrate. An excess of $\mathrm{TiO}_{2}$ solution was applied to the substrate, which in turn was loaded onto the spin coater. Centrifugal forces then force the solution to run away, leaving a very thin layer of $\mathrm{TiO}_{2}$. Highly volatile solvents were best for this purpose. The higher the rotation speed, the thinner the deposited film. Final thickness was also dependent on the type of the solvent and solute concentration. A blocking layer of $\mathrm{TiO}_{2}$ was deposited on FTO by this technique in the fabrication of solid-state dyesensitized solar cells (ssDSCs). A model WS-400B-6NPP/LITE spin coater was used for all spin coating preparations.

Procedure: Ethanol $(40 \mathrm{~mL})$ with $1 \mathrm{~mL}$ of deionised water was stirred magnetically at room temp and 5 drops of conc. $\mathrm{HNO}_{3}$ (70\%) was added. Titanium tetraisopropoxide $(3 \mathrm{~mL})$ was then added drop-wise, resulting in a solution of $\mathrm{pH} 2$. Conc. $\mathrm{HNO}_{3}$ acid (2 drops) was added and spin coating was done at a rate of 1000 rpm for 50 secs. The thickness of the $\mathrm{TiO}_{2}$ layer was controlled by using 2 layers of Scotch tape as a mask. Finally, the substrate was heated to $100^{\circ} \mathrm{C}$ for 60 minutes, and then sintered at $450^{\circ} \mathrm{C}$ for 45 minutes. 


\subsubsection{Spray pyrolysis}

A very thin layer of semiconductor (nano-domain) was deposited by spray pyrolysis. The concentration of the precursor solution was most important for this process. A dilute solution of the chosen solute was sprayed onto a heated surface, where the solvent evaporates. Various kinds of semiconductor oxides and sulphides $\left(\mathrm{TiO}_{2}\right.$, $\mathrm{SnO}_{2}, \mathrm{CdS}, \mathrm{CuS}$, and $\mathrm{ZnS}$ ) can be coated onto highly temperature resistant conducting substrates by this method.

The solvent initially vaporises, leaving solid material on the surface. Further heating creates a diffused uniform layer. The thickness of the desired material can be controlled by the concentration of the precursor solution, type of solvent, the size of the orifice nozzle, radius of the droplet of the solution, temperature, the distance from the head of pyrolyser to the intended heated conducting substrate, and the number of spraying events. For 10 sprays, the thickness of $\mathrm{TiO}_{2}$ was typically 100$150 \mathrm{~nm}$ and particle size was approximately 5-10 nm. The $\mathrm{TiO}_{2}$ layer needs to be uniform and of correct thickness.

Procedure: The blocking $\mathrm{TiO}_{2}$ layer was prepared by spray pyrolysis. Titanium diisopropoxide bis-acetylacetonate (TAA) solution was prepared by diluting TAA, $75 \%$ in isoproponol (Aldrich) in isopropanol to a 1:9 volume ratio. The solution was stored in a refrigerator when not in use. A hot plate was kept in the fume hood for spray pyrolysis. The FTO substrates were heated to $450^{\circ} \mathrm{C}$, with a 15 minutes ramp time, and left for 5 minutes to reach temperature. One round was sprayed every 10 secs, for 2 minutes (12 rounds). For each full master plate, 5 sprays were used, one in each corner and one in the middle. The nozzle of the pyrolyser was held approximately $100 \mathrm{~mm}$ from the surface. Sprays were quick and as hard as possible. Substrates were left on the hot plate for a further 5 minutes at $450^{\circ} \mathrm{C}$ before cooling to room temperature. The pyrolyser head was stored in either ethanol or methanol when not in use. This procedure was obtained from Dr. Udo Bach's research laboratory (Monash University, Melbourne, Australia). 


\subsubsection{Preparation of $\mathrm{TiO}_{2}$ mesoporous layer}

The mesoporous $\mathrm{TiO}_{2}$ layer is coated onto transparent conducting glass (TCO) such as fluorine doped tin oxide (FTO). The mesoporous oxide layer $(\sim 5.5 \mu \mathrm{m})$ is comprised of $\mathrm{TiO}_{2}$ nanoparticles in a network fashion which are sintered for better inter-necking among $\mathrm{TiO}_{2}$ particles $(\sim 20 \mathrm{~nm})$ and to develop good electronic conduction. The porosity of the film is roughly $50-60 \%$. Films having high porosities lead to less interconnection among $\mathrm{TiO}_{2}$ particles and decrease the charge collection efficiency.

\subsubsection{Doctor-blade technique}

Doctor blade coating is a technique which has been used to prepare mesoporous $\mathrm{TiO}_{2}$ layer. This technique is roll-to-roll, cost effective and compatible.

\section{Procedure:}

Pre-cleaned $7 \mathrm{~cm} \times 3 \mathrm{~cm}$ FTO slides were stored in a dried air filter box. Two layers of Scotch tape were put parallel on two sides of the FTO with a $1 \mathrm{~cm}$ gap in between, and then NanoTi Oxide (T) paste was applied on the conductive side of the FTO. This paste was carefully doctor bladed uniformly by using a glass tube. Rolling was not repeated on the same film. After a few minutes, the $\mathrm{TiO}_{2}$ became transparent. Another layer of $\mathrm{TiO}_{2}$ paste was then applied, then a reflective layer of Ti nanooxide 300 (300 nm size $\mathrm{TiO}_{2}$ ) was applied. The slides were placed on a hot plate until the coating turned to a yellow colour. The slides were cut into $1.5 \mathrm{~cm} \times 3 \mathrm{~cm}$ rectangles and put into a muffle furnace at $500^{\circ} \mathrm{C}$ for 30 minutes. The yellow organic layer turns white after cooling. The hot plate which was used for sintering was supplied by Harry Gestigkeit GmbH. The sintering of these blocking/ mesoporous $\mathrm{TiO}_{2}$ layer was done with the standard temperature program. 


\subsubsection{Dye sensitization}

The surface of the nanocrystalline oxide layer is coated with a monolayer of various intended charge transfer dyes.

\section{Procedure:}

A $0.3 \mathrm{mM}$ solution of each dye was prepared in $50 \%(\mathrm{v} / \mathrm{v})$ acetonitrile/ t-butanol. 50 $\mathrm{ml}$ of Z907 dye solution was placed in an ultrasonic bath or magnetically stirred overnight to ensure dissolution. In the case of zinc-porphyrin dye, a $0.2 \mathrm{mM}$ THF solution was prepared. After heating to $120^{\circ} \mathrm{C}, \mathrm{TiO}_{2} / \mathrm{FTO}$ glass was immersed into the dye solution for 20-24 hrs. Two working electrodes were put into the dye solution at the same time, facing in opposite directions. They were then washed with acetonitrile and dried with a $\mathrm{N}_{2}$ gas stream.

\subsubsection{Impregnation of hole transporting material in dyed $\mathrm{TiO}_{2}$}

To form the hole transporting material (HTM) into dyed $\mathrm{TiO}_{2}$ nanomatrix, in situ photo electrochemical polymerization had been employed. This was done according to the procedure in section 2.2.2. Photo-electrochemical polymerization was carried out with different light intensities varied from 1 to $200 \mathrm{~mW} / \mathrm{cm}^{2}$ using optical filters, dopant anions, thicknesses of $\mathrm{TiO}_{2}$, oligomers, and PEDOT growth time, in order to find optimal conditions.

\subsubsection{Preparation of counter electrode}

\subsubsection{Sputter coating}

Sputter coating is a technique where a thin layer of metal (e.g Pt, $\mathrm{Au}, \mathrm{Pd}, \mathrm{Ni}, \mathrm{Ti}$ ) is deposited on a desired substrate. Even metals of high melting point can be used. Various types of sputtering exist: magnetron, reactive, ion beam, gas flow, high 
power impulse magnetron, high target utilization, and ion assisted sputtering are some examples.

The magnetron method produces better adhesion to the substrate than the evaporation method. This is a great advantage of this technique. However, it can be difficult to achieve a structured film, a disadvantage. Only magnetron sputtering for the preparation of $\mathrm{Au} / \mathrm{Pt}$ on ITO counter electrodes will be discussed here.

Procedure: ITO glass was washed with ethanol followed by UV ozone cleaning. A thin layer of gold or platinum was then sputtered on to the ITO using a Dynavac Magnetron Sputter Coater Model SC100MS. An argon pressure of $2 \times 10^{-3} \mathrm{mbar}$ and sputtering current of $50 \mathrm{~mA}$ were used. The deposition rate was $2 \AA / \mathrm{s}$ to produce a 10-30 Å thickness of Au or Pt.

\subsubsection{Metal evaporation coating}

A metal evaporator supplied by AVT Services Limited (SQM-160 Rate/thickness monitor) was employed for this work. Metal coating was done at $10^{-5}$ mbar pressure and $1600-1700^{\circ} \mathrm{C}$. The thickness of coating was controlled by the applied current.

\subsubsection{Ionic liquid treatment on photo-anode}

Before assembly of the devices, photo-anode films were rinsed with acetonitrile and subsequently treated for $24 \mathrm{hrs}$ with 1-ethyl-3-methylimidazolium bistrifluoromethanesulfonamide (EMITFSI) containing $0.2 \mathrm{M}$ lithium bistrifluoromethanesulfonamide and 0.2 M 4-tert-butylpyridine (t-BP). The sandwichtype devices were completed by clipping the counter electrode to the photo-anode. 


\subsubsection{Assembly of the solid-state dye-sensitized solar cells}

Solid-state dye-sensitized solar cells (comprising both dye-sensitized $\mathrm{TiO}_{2}$ photoanodes and counter electrodes) were assembled and held together by a metal clip (Figure 2.3).

\section{4 Device fabrication of liquid dye-sensitized solar cells}

In order to fabricate the liquid DSCs, the working electrode was prepared by spin coating $\mathrm{a} \sim 100 \mathrm{~nm}$ layer of $\mathrm{TiO}_{2}$ using titanium-isopropoxide as a precursor solution. The $\mathrm{TiO}_{2}$ films were then sintered at $500^{\circ} \mathrm{C}$ for 30 minutes. Films of various thicknesses were prepared by masking with different number of layers of Scotch tape. A transparent $\mathrm{TiO}_{2}$ layer on FTO glass was prepared by a doctor blading (using Solaronix Nanoxide-T paste). In some cases a thick scattering layer of $\mathrm{TiO}_{2}$ (Solaronix paste, $300 \mathrm{~nm}$ particle size) was created. This paste was deposited on top and sintered for a further 30 minutes at $500^{\circ} \mathrm{C}$. The electrode was then immersed into a $0.3 \mathrm{mM}$ Z907 dye solution for $24 \mathrm{hrs}$.

The counter electrode had a $30 \mathrm{~nm}$ thickness of Pt on ITO coated glass and was cut with a glass cutting knife from the uncoated side. The cut electrodes were placed in water (cooling) and a small hole was drilled with a diamond drill. Electrodes were then washed with distilled water, dried with an $\mathrm{N}_{2}$ gas stream and placed in a $120^{\circ} \mathrm{C}$ oven. A spacer (Himilan, $25 \mu$ ) was put between the counter electrode and dyed- $\mathrm{TiO}_{2}$ for filling of electrolyte. These spacers were made of polymer that melts at $100^{\circ} \mathrm{C}$, sealing the cell. The electrolyte was a mixture of 0.1 M LiI, 0.6 M DMPII, and 0.05 $\mathrm{M} \mathrm{I}_{2}$ and $0.5 \mathrm{M} \mathrm{t}$-BP. After sealing both working and counter electrode, the device was masked with a black pen. The active area of the device was $\sim 0.300 \mathrm{~cm}^{2}$. 


\subsection{Characterisation of the photovoltaic device}

The solar cells performances (both ssDSC and liquid DSC) are evaluated by photocurrent/ photovoltage $(\mathrm{J}-\mathrm{V})$ measurement. Four parameters will be determined while measuring the $J-V$ curve of the device. They are as follows:

i. Open-circuit voltage $\left(V_{\mathrm{oc}}\right)$ : This is the cell voltage when current within the solar cells equal to zero.

ii. Short-circuit current $\left(J_{\mathrm{sc}}\right)$ : This is the solar cells photocurrent when measured at zero voltage.

iii. Fill Factor (FF) : It is the ratio of maximum power output $\left(\mathrm{P}_{\mathrm{m}}\right)$ to the product of Short-circuit current $\left(J_{S C}\right)$ and Open-circuit voltage $\left(V_{\mathrm{oc}}\right)$

Therefore, $\mathrm{FF}=\mathrm{P}_{\mathrm{m}} /\left(J_{\mathrm{sc}} \times V_{\mathrm{oc}}\right)$

Which can be written also as $\mathrm{FF}=\left(\mathrm{I}_{\mathrm{m}} \times \mathrm{V}_{\mathrm{m}}\right) /\left(J_{\mathrm{sc}} \times V_{\mathrm{oc}}\right)$

Where as $\mathrm{I}_{\mathrm{m}}=$ Current at maximum power output

$\mathrm{V}_{\mathrm{m}}=$ Voltage at maximum power output.

iv. Energy conversion efficiency $(\eta)$ : It is the ratio of maximum power output to the incident radiation power $\left(\mathrm{P}_{\text {in }}\right)$

$$
\begin{aligned}
\eta & =\mathrm{P}_{\mathrm{m}} / \mathrm{P}_{\text {in }} \\
& =\left(J_{\mathrm{sc}} \times V_{\mathrm{oc}} \times \mathrm{FF}\right) / \mathrm{P}_{\text {in }}
\end{aligned}
$$

The efficiency of the device depends on all three parameters $J_{\mathrm{sc}}, V_{\mathrm{oc}}$ and FF. The higher these values, the higher will be the efficiency of the device.

\subsubsection{Current voltage measurement (J-V)}

The photoenergy conversion efficiencies of fabricated devices were measured using a solar simulator illumination of air mass AM 1.5 and $100 \mathrm{~mW} / \mathrm{cm}^{2}$ calibrated white light. This afforded all information about current density, open circuit voltage, fill factor, and efficiency. Light output from the solar simulator was constantly monitored and controlled via a silicon detector and feedback control located just under the main collimating lens of the solar simulator. Calibration was performed 
when the lamp was first started or when in doubt of the correct light intensity. The Si calibration solar cell was never kept under bias in order to avoid damage. The solar simulator set-up is shown in Figure 2.4.

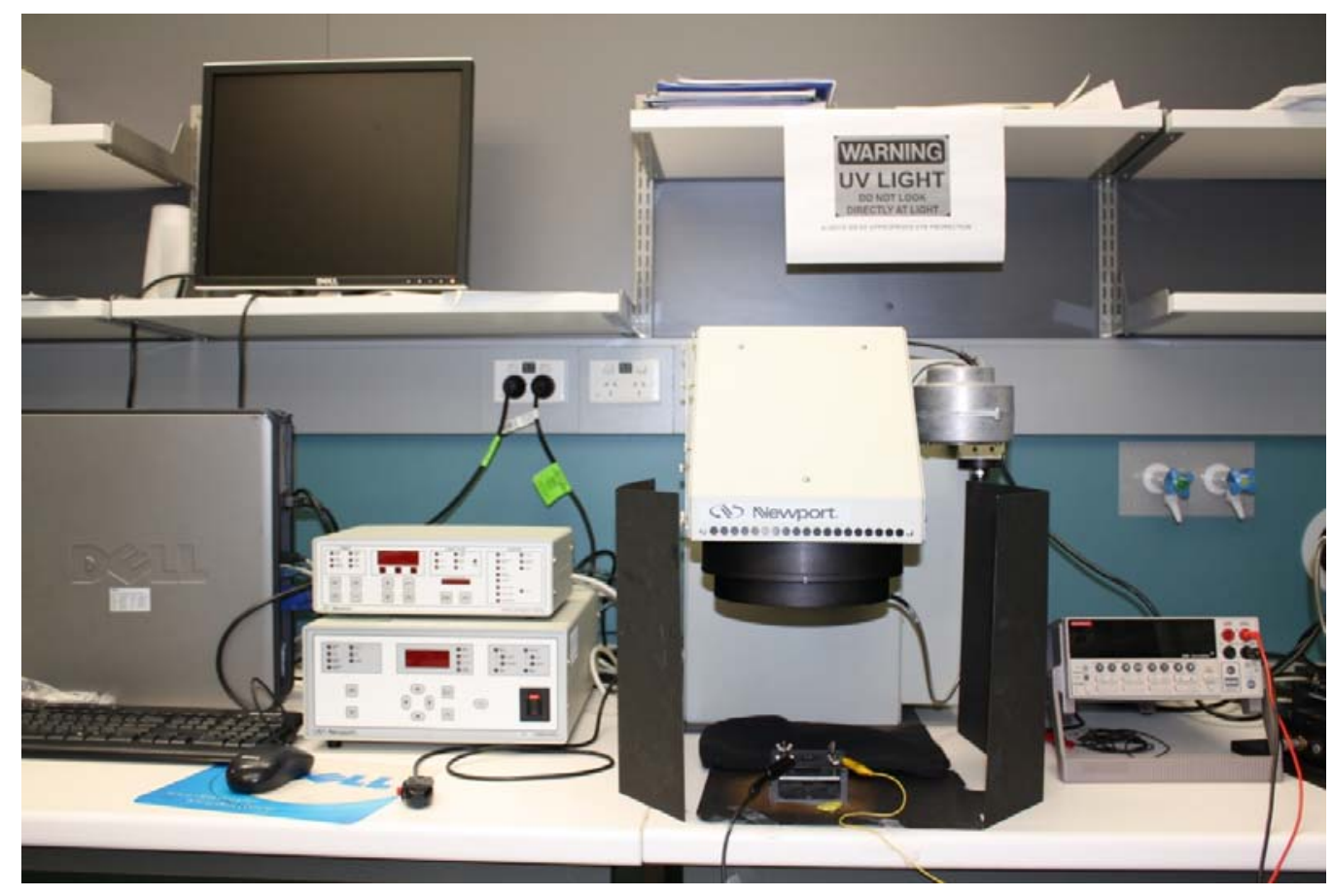

Figure 2.4: Solar simulator set-up for measurement of photo-energy conversion efficiency.

\subsubsection{Incident photon to current conversion efficiency measurement}

Incident photon to current conversion efficiency (IPCE) is the ratio of photocurrent to incident photon flux as a function of excitation wavelength. IPCE is very informative when a new material is used in the solar cells. Before IPCE measurements, calibration with a standard silicon photo detector (Pecell technologies, Inc Japan, BS-520, S/N 091) was performed. 
The IPCE equipment set-up is shown in Figure 2.5. IPCE was calculated by using the formula $\mathrm{IPCE}=1240 / l \times J_{\mathrm{sc}} / \mathrm{P}_{\text {in }}$ (where $l$ is the wavelength in $\mathrm{nm}, J_{\mathrm{sc}}$ is the shortcircuit current, $\mathrm{P}_{\text {in }}$ is the incoming light power).

$\operatorname{IPCE}(\lambda)=\operatorname{LHE}_{(\lambda)} \times \Phi_{\text {inj }} \times \Phi_{\text {col }}$

Whereas LHE $_{(\lambda)}=$ Light harvesting efficiency of active material; $\Phi_{\text {inj }}=$ Charge injection efficiency (charge injects via dye to $\mathrm{TiO}_{2}$ ); $\Phi_{\text {col }}=$ Charge collection efficiency at the external electrode.

IPCE is also called as external quantum efficiency (EQE). The IPCE values are obtained by measuring the photocurrent response while changing the wavelength of the incident light.

This IPCE set-up consisted of the following components:

- $300 \mathrm{~W}$ Xe lamp +3 grating monochromatic.

- $\quad$ Automatic filter wheel with 5 cut-off filters.

- Fibre coupling and focusing assembly $0.5 \mathrm{~cm}^{2}$ spot size

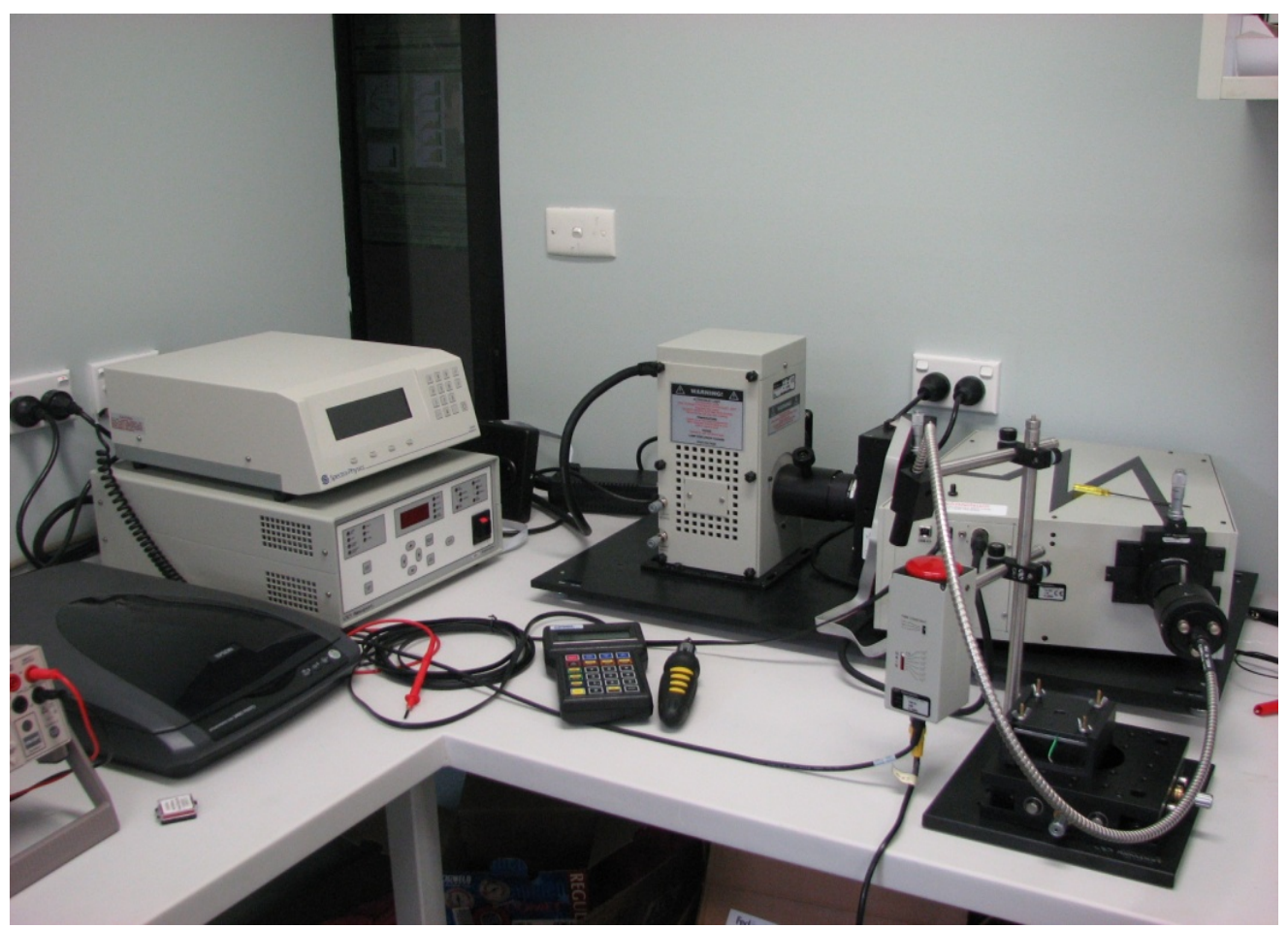

Figure 2.5: IPCE: photon to current conversion efficiency equipment. 


\subsubsection{UV-Vis-NIR spectroscopy}

Ultraviolet-visible-near-infrared (UV-Vis-NIR) spectroscopy is employed to measure the optical absorbance and reflectance 200-3300 $\mathrm{nm}$ wavelength range. The absorbance spectra were recorded in the range of $300-2800 \mathrm{~nm}$ to give the information on the doping level, conductivity, conjugation length of polymer and absorption spectra of dye (used as photo-sensitizer). This was employed to get the information about the dye/ PEDOT absorption, loss mechanism and optical transparency and the amount of PEDOT present in photo-anode. The doping level of PEDOT has been examined when the PEDOT is grown in photo-anode under various conditions.

\subsubsection{Raman spectroscopy}

Raman spectroscopy is a powerful instrumental technique. It is very popular due to it being non-destructive and having easy sample preparation. It has been widely used to study the various doping processes and oxidation states of conducting polymers prepared by the electrochemical method.

Raman spectra of PEDOT deposited on the photo-anode (working electrode) were measured here using a Jobin Yvon Horiba HR800 Raman spectrometer with Labspec software. The Raman spectrum of a PEDOT film was recorded using $785 \mathrm{~nm}$ excitation. The peaks were deconvulated using Gaussian fitting, and Raman intensities are reported in a.u (arbitrary unit). Raman mapping was done with an $x-y$ resolution of $1 \mu \mathrm{m}$ over a particular area of the PEDOT film.

The different type and quantity of the dopant in a conducting polymer can significantly affect its physical properties such as conductivity. Moreover, by using different dopant anions during photo-polymerization, their effect on conductivity and the performance of solid-state dye-sensitized solar cells can be investigated. In this study, $\mathrm{ClO}_{4}{ }^{-}$has been examined as dopant. 


\subsubsection{MALDI-ToF- mass spectrometry}

Matrix assisted laser desorption ionisation (MALDI) coupled to Time of Fight (ToF) was employed to measure the molecular distribution of PEDOT on the photo-anodes. The purpose of using this technique was to generate a mass spectrum which can give information about repeating chain units, end groups, and the molecular weight distribution of the various amounts of PEDOT polymer on the photo-anodes. Photoanodes were prepared by our standard photo-electrochemical polymerization technique.

Solventless MALDI technique involves the use of only solid analyte and matrix during sample preparation. They found that Solventless MALDI of solid polyaniline resulted in mass spectra that were similar to spectra of the soluble oligomers with some larger molecular weight oligomers which were detected by the direct laser desorption ionization method. Thus, solventless MALDI appears to be a promising new technique for the mass spectrometric analysis of PEDOT.

PEDOT (really poly bis-EDOT) samples were analysed by the new technique of solventless MALDI, using a Shimadzu Axima Confidence instrument. Intact solar cell films of approximately $1 \mathrm{~cm}^{2}$ were scraped from their glass backing with a razor blade into a small agate mortar. Approximately 50 times the mass of 2, 5dihydroxybenzoic acid (DHB) was added and the contents mixed with a spatula, then ground with an agate pestle to a fine powder. A small quantity of the powder was picked up on the tip of a fine spatula and smeared well onto the MALDI target. Compressed air was then used to blow any loose material away. Spectrometer power settings varied between 72 and 96 arbitrary units and $m / z$ optimization settings were set to approximately $50 \%$ of the highest detectable mass for each sample. Spectra were collected in 1000 profile lots with 10 laser shots per profile, and then smoothed using a 10 channel filter width and the baseline subtracted with a filter width of 500 channels. 


\subsubsection{Morphology studies by SEM and AFM}

The morphology of photo-anodes was characterised by both atomic force microscopy (Asylum Research, Atomic force microscope, MFP 3D), scanning electron microscopy, and SEM-Energy Dispersive X-ray Analysis (JEOL, JSM -7500 FA) instruments. The morphology of the polymer and semiconductor affects photovoltaic performance. The porosity, morphology, and filling effect of hole transporting material which was impregnated by photo-electrochemical method into the dyed nanomatrix of $\mathrm{TiO}_{2}$ can be fully understood by AFM and SEM techniques.

The surface and cross-sectional morphologies of SEM images of PEDOT grown under different conditions have been determined. AFM images of each layer of the photo-anodes were obtained, such as compact layer $\left(\mathrm{TiO}_{2}\right)$ where the pores between these particles can be studied; and secondly with the non-porous electrodes the smoothness of the films can be determined.

Attempts were made to correlate the AFM images with the surface morphology measured by SEM. For this, AFM images, and then SEM images were taken in the same place on the photo-anode (and vice versa). When the images of the photoanode of PEDOT grown for 30 minutes were mapped, it was observed that some areas were dark and some were white, so the SEM was zoomed in to obtain a 2-2.5 $\mu \mathrm{m}$ image of both areas to see the differences. SEM and AFM images were also taken of all photo-anodes where different amounts of PEDOT were photoelectrochemically deposited. 


\section{FABRICATION OF SOLID-STATE DYE- SENSITIZED SOLAR CELLS}




\section{1 Introduction}

In this chapter, the in situ photo-electrochemical polymerization (PEP) will be discussed and its use as a powerful technique for the fabrication of solid-state dyesensitized solar cells (ssDSCs). This technique will be explored in model studies for a range of ruthenium dyes and different hole transporting materials (HTMs). Various light intensities during photo-electrochemical polymerization are examined, various dopant anions, various thicknesses of $\mathrm{TiO}_{2}$ (both blocking layer and mesoporous layer), different oligomer starting material, and a range of polymerization times. The use of different solvents in the polymerization of poly (3, 4-ethylenedioxythiophene) (PEDOT) is also described. How all these different conditions during photoelectrochemical polymerization of PEDOT influenced the performances of the fabricated ssDSCs will also be discussed.

This Chapter also provides a detailed investigation of the parameters which are necessary for the dyes and HTMs for successful in situ photo-electrochemical polymerization. More specifically, the determination of the surface coverage of the dyes on $\mathrm{TiO}_{2}$, the molar extinction coefficient of the dyes, and the HOMO and LUMO energy levels of the dyes are also established.

In liquid dye-sensitized solar cells, typically $\mathrm{I}^{-} / \mathrm{I}_{3}{ }^{-}$redox couple is used a redox mediator and a volatile solvent e.g. acetonitrile. Chiba et al. [1] reported 11.2\% efficiency for DSCs. Even if these DSCs are less expensive, one must address issues such as sealing the cell, a challenge due to the volatility and corrosive nature of the electrolyte. These problems are exacerbated at higher temperature [2]. Several attempts had been made by scientists to overcome these problems. In another approach, p-type inorganic semiconductors, hole transporting materials (HTMs), conducting organic polymers, have been used as an alternative solid-state redox mediator to replace the liquid electrolyte.

The impregnation of this HTM into nanostructured dyed $\mathrm{TiO}_{2}$ has been achieved using the photo-electrochemical polymerization method. This approach has led to devices with up to $2.8 \%$ efficiency [3]. While this efficiency is promising, it is 
below the efficiency of liquid dye-sensitized solar cells, and now overall device performance need to be improved for commercialisation. The low efficiencies of this kind of ssDSCs need to be investigated.

In this Chapter, the conducting organic polymer, PEDOT, will be used as the hole transporting material [4-6]. Initially Yanagida et al. had proposed polypyrrole (PPy) as an HTM using in situ photo-electrochemical polymerization, but the resulting ssDSC had an efficiency of only $0.5 \%$ [7]. The poor performance of the polypyrrole conducting polymer was due to its strong absorbance in the UV-Visible region, which restricted the photon harvesting by dye. The conducting polymer $(\mathrm{CP})$ must be transparent in the UV-Vis spectral range of the dye so that none of the light captured from the dye (which is anchored to the $\mathrm{TiO}_{2}$ surface). If the CPs absorbs light it should be as efficient at electron injection as the dye does within DSCs. So, an alternative stable conducting polymer/electroactive polymer PEDOT has been used as a HTM since it is more transparent in the UV-Vis range. EDOT (3, 4ethylenedioxythiophene) can not be photopolymerized because of its high oxidation potential $\left(\sim 1.0 \mathrm{~V}\right.$ vs. $\left.\mathrm{Ag} / \mathrm{Ag}^{+}\right)$as compared to oxidation potential of ruthenium based dye (Z907, $\sim 0.59 \mathrm{~V}$ vs. $\mathrm{Ag} / \mathrm{AgCl})$, so an oligomer bis-EDOT ( $0.4 \mathrm{~V}$ vs. $\mathrm{Ag} / \mathrm{AgCl})$ has a lower oxidation potential than the dye and can readily be photoelectrochemically deposited into the dyed $\mathrm{TiO}_{2}$ nano-matrix [3].

The structure of the Z907 dye (cis-Bis (isothiocyanato) (2, 2'-bipyridyl-4, 4'dicarboxylato) (4, 4'-di-nonyl-2'-bipyridyl) ruthenium (II) to be employed is presented in Figure 3.1. This is a hydrophobic dye because of presence of long (C9) alkyl chain. It is efficient sensitizers which can sensitize the wide band gap titania up to $750 \mathrm{~nm}$ wavelength. This Z907 dye improves the stability and repels any water molecules present in ssDSCs. This dye tightly adsorbs onto the surface of $\mathrm{TiO}_{2}$. This dye has high molar extinction coefficient $\left(12500 \mathrm{M}^{-1} \mathrm{~cm}^{-1}\right.$ at $\left.513 \mathrm{~nm}\right)$ which makes an ideal photo-sensitizer for ssDSCs. This dye shows metal to ligand charge transfer transition (MLCT) which is responsible for the light absorption in the visible wavelength range $(\sim 450-550 \mathrm{~nm})$. 


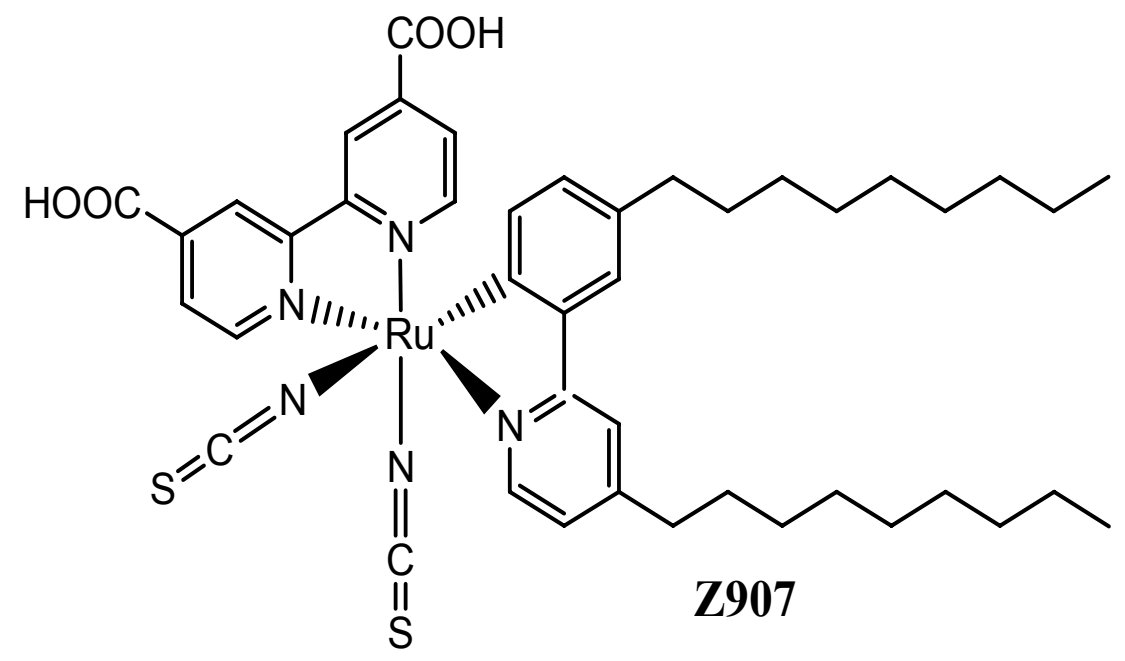

Figure 3.1: The structure of the ruthenium based (hydrophobic) Z907 dye.

\section{2 Experimental}

\subsubsection{Calculation of HOMO-LUMO energy levels, surface coverage, and molar extinction coefficient of Z907 dye}

\subsubsection{Cyclic voltammogram (CV) of Ruthenium dye}

$0.10 \mathrm{mM}$ dye (e.g. Z907) was dissolved in 0.10 M TBAP in acetonitrile (5 ml). CV was performed with a scan rate of $100 \mathrm{mV} / \mathrm{s}$, initial and final potential $=0 \mathrm{mV}$, upper limit $1000 \mathrm{mV}$, lower limit $-500 \mathrm{mV}$, using a glassy carbon working electrode, $\mathrm{Ag} /$ $\mathrm{Ag}^{+}$reference electrode and Pt mesh as an auxiliary electrode.

\subsubsection{CV of dye-sensitized $\mathrm{TiO}_{2}$ film}

Using a doctor blading method, $\mathrm{TiO}_{2}$ films were made on FTO glass using Solaronix $\mathrm{T} \mathrm{TiO}_{2}$ paste. The $\mathrm{TiO}_{2}$ films were sintered using a standard program at $325^{\circ} \mathrm{C}(30$ $\min ), 375^{\circ} \mathrm{C}(15 \mathrm{~min}), 450^{\circ} \mathrm{C}(30 \mathrm{~min})$, and a maximum of $500^{\circ} \mathrm{C}(30 \mathrm{~min})$. For sensitization, different dye solutions containing $(0.3 \mathrm{mM}$ dye in acetonitrile and t- 
butanol 1:1 ratio) were used. $\mathrm{The}^{\mathrm{TiO}_{2}}$ films were reheated at $120^{\circ} \mathrm{C}$ for one hour to remove any moisture from the $\mathrm{TiO}_{2}$ film. They were then immersed in dye solution for 24 hours. CV growth was performed with a scan rate of $100 \mathrm{mV} / \mathrm{s}$, initial and final potential $=0 \mathrm{mV}$, upper limit $1000 \mathrm{mV}$, lower limit $-500 \mathrm{mV}$, using dyed $\mathrm{TiO}_{2}$ films as the working electrode, $\mathrm{Ag} / \mathrm{Ag}^{+}$as reference electrode and Pt mesh as auxiliary electrode.

\subsubsection{Standard Ferrocene CV}

A $1.0 \mathrm{mM}$ ferrocene solution was prepared in acetonitrile containing 0.10 M TBAP in $5 \mathrm{ml}$ of acetonitrile and the cyclic voltammogram was recorded as above.

\subsubsection{UV-Vis spectra of dyes to determine optical band gap}

The UV-Vis spectrum of the ruthenium based dye Z907 was measured in ethanol solvent to determine the optical band gap. The dye solution was prepared using ethanol. A Shimadzu UV-1601 spectrophotometer was employed. The baseline was corrected using ethanol solvent. In the case of dye sensitised $\mathrm{TiO}_{2}$ films, the base line was corrected using an $\mathrm{FTO}_{-} \mathrm{TiO}_{2}$ film.

\subsubsection{UV-visible spectral studies}

Optically transparent conducting FTO glasses were cut into $4 \times 8 \mathrm{~cm}$ pieces and cleaned with snow cleaning (liquid $\mathrm{CO}_{2}$ ) followed by $\mathrm{UVO}_{3}$ cleaning for 18 minutes. A layer of $\mathrm{TiO}_{2}$ paste was coated by a doctor blading method with one scotch tape masking to produce a $\mathrm{TiO}_{2}$ film of $\sim 3 \mu \mathrm{m}$ thicknesses. All these films were sintered overnight with a standard temperature program described in section 3.2.1.2. These substrates were then cut into $1.5 \mathrm{~cm} \times 3 \mathrm{~cm}$ pieces and again sintered for $2 \mathrm{hrs}$ to remove excess moisture from the films, and put into a solution of ruthenium dye Z907 for $24 \mathrm{hrs}$ (see section 2.3.4 (Chapter 2) for solution preparation). Prior to measuring the UV-Vis spectra of these sensitized films they were treated with one 
drop of propylene carbonate to better match their refractive index and remove interference fringes from the baseline.

\subsubsection{Fabrication of PEDOT-based solid-state dye-sensitized solar cells}

\subsubsection{Preparation of blocking layer of $\mathrm{TiO}_{2}$ on FTO glass}

FTO glass was cut and then cleaned by snow cleaning (liquid $\mathrm{CO}_{2}$ ), then $\mathrm{UVO}_{3}$ cleaning. A compact/blocking $\mathrm{TiO}_{2}$ layer was deposited by spin-coating onto the FTO glass. The blocking/compact layer of $\mathrm{TiO}_{2}$ film was sintered at $450^{\circ} \mathrm{C}$ for 30 minutes. The purpose of this blocking layer was to avoid an electrical contact between the HTM and the FTO. This layer was also being deposited by spray pyrolysis. The details of these two procedures have been described in section 2.3.4 (Chapter 2).

\subsubsection{Preparation of mesoporous $\mathrm{TiO}_{2}$ layer on blocking layer}

A $\sim 5.5 \mu \mathrm{m}$ thick nanocrystalline $\mathrm{TiO}_{2}$ film was made on top of the blocking layer by a doctor-blading technique using commercial Solaronix nanoxide-T paste, and sintered up to $500^{\circ} \mathrm{C}$ with the standard temperature program. The thickness of $\mathrm{TiO}_{2}$ was varied with the number of scotch tape put as a mask.

\subsubsection{Preparation of Z907 dye solution for sensitization of working electrode}

$0.3 \mathrm{mM}$ of Z907 dye was prepared in acetonitrile and t-butanol (50:50 by volume). This dye solution was then either ultrasonicated in an ultrasonic bath for half an hour or magnetically stirred overnight to make a clear and transparent dye solution.

\subsubsection{Sensitization of $\mathrm{TiO}_{2}$ film}

After sintering at $500^{\circ} \mathrm{C}$ the $\mathrm{TiO}_{2}$ films were cooled to $120^{\circ} \mathrm{C}$, and sensitized for 20 24 hrs. All $\mathrm{TiO}_{2}$ films were sensitized in the dark. After sensitization, these 
substrates were washed with acetonitrile to remove agglomeration or excess of dye on the $\mathrm{TiO}_{2}$ surface. The films were cleaned and dried by blowing with $\mathrm{N}_{2}$ gas.

\subsubsection{In situ photo-electrochemical polymerization of bis-EDOT}

To form the hole transporting material (HTM), Z907 dyed $\mathrm{TiO}_{2} / \mathrm{FTO}$ substrates were immersed in a solution of $0.10 \mathrm{M} \mathrm{LiClO}_{4}$ and $0.01 \mathrm{M}$ bis-EDOT in acetonitrile and illuminated from the mesoporous dyed $\mathrm{TiO}_{2}$ side by a SoLUX lamp (12 V, $50 \mathrm{~W}$ WaKO, Japan). The intensity and wavelength of light were $22 \mathrm{~mW} / \mathrm{cm}^{2}$, and $>485$ $\mathrm{nm}$. The intensity of the light was measured by the help of light meter (Model LI250A Light Meter, LI-COR) and the intensity of light was varied by using neutral density filters.

Photo-electrochemical polymerization was carried out under potentiostatic conditions in a three-electrode one-compartment electrochemical cell. The $\mathrm{Z907}$ dyed $\mathrm{TiO}_{2}$ substrate was the working electrode, Pt mesh was the counter electrode, and $\mathrm{Ag} / \mathrm{AgCl}$ was the reference electrode. A constant potential $(0.2 \mathrm{~V}$ vs. $\mathrm{Ag} / \mathrm{AgCl})$ was applied and the current monitored using eDAQ software.

PEDOT polymer was grown throughout the mesoporous dyed $\mathrm{TiO}_{2}$ film as photoelectrochemical polymerization of bis-EDOT proceeded. The total charge transferred during polymerization was calculated by integration of the polymerization current. The Energy level diagram and schematic of various path of electron transfer in ssDSCs is shown in Figure 3.2 where as the proposed reaction mechanism of the in situ photo-electrochemical polymerization of bis-EDOT to PEDOT is shown in Figure 3.3 respectively. In the first step (1), the dye is photo-oxidised by injecting an electron from its photo-excited state into the $\mathrm{TiO}_{2}$ conduction band $(\mathrm{CB})$ and then the oxidised dye cations oxidise the bis-EDOT molecule. The concentration of oxidised dye is dependent on the wavelength of light and the light intensity used for photopolymerization. The effect of externally applied bias potential is to remove electrons from $\mathrm{TiO}_{2}$ and thus prevent recombination with dye cations. The electron injection from PEDOT to dye cation $\left(\mathrm{S}^{+}\right)$may be controlled by the driving force as determined 
by the difference in oxidation potential between PEDOT and dye. The oxidation potential of EDOT oligomers decreases with increase of EDOT units.

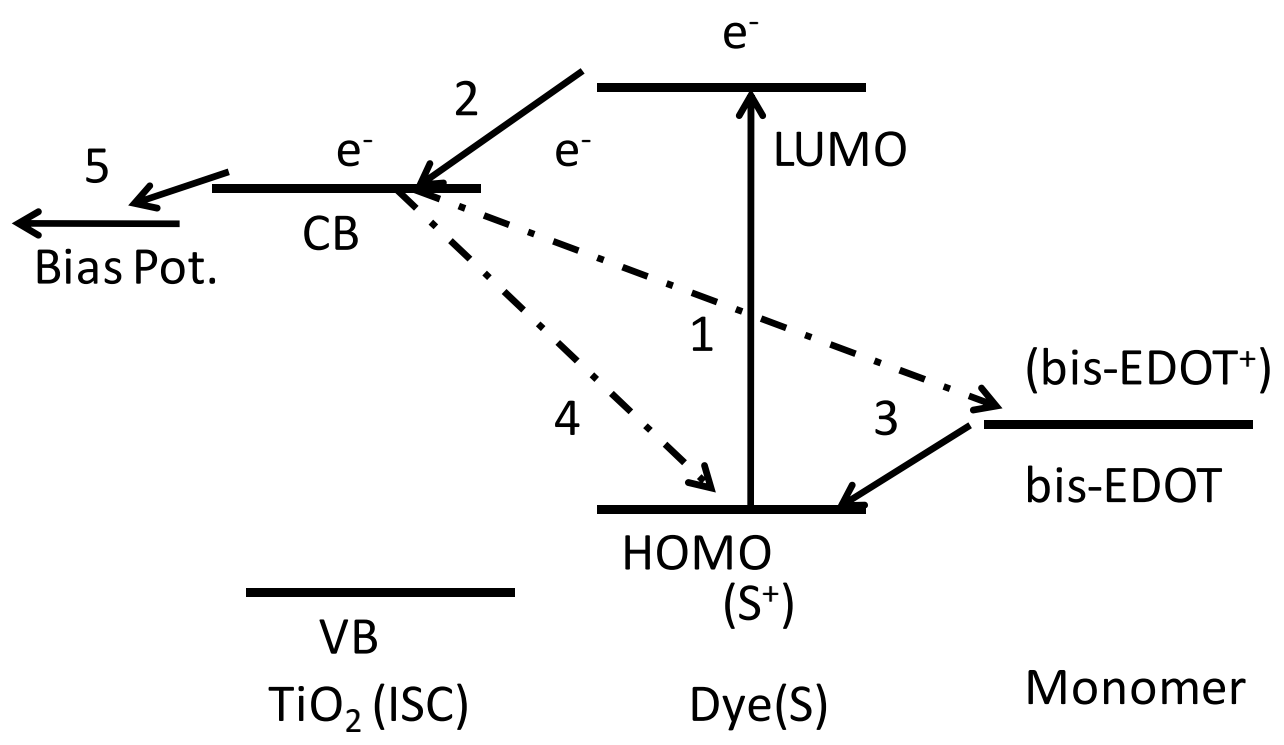

Figure 3.2: Energy level diagram and schematic of various path of electron transfer in ssDSCs (Steps 3 and 4 are loss mechanism).

In order to understand the effect of the photo-electrochemical polymerization on the performance of solid-state DSCs, information on the energy levels of the various species involved including $\mathrm{TiO}_{2}$ (inorganic semiconductor ISC), dye sensitizer (S), monomer (M), polymer (P), and counter electrode (CE) are presented in Figure 3.2. The photo-sensitizer (dye) absorbs photons from a broadband white light source with energy greater than its HOMO-LUMO band gap and transfers electrons into the conduction band of the titania. The photo-oxidised dye is reduced by electron transfer from the monomer, initiating polymerization. 
Proposed mechanisim for formation of PEDOT within photo-anode

1. Dye $\stackrel{h v}{\longrightarrow}$ Dye $^{*} \quad$ (Photoexcitation)

2. $\mathrm{Dye}^{*}+\mathrm{TiO}_{2} \longrightarrow \mathrm{Dye}^{+}+\mathrm{e}^{-}{ }_{\mathrm{TiO} 2}$ (Injection)

3.
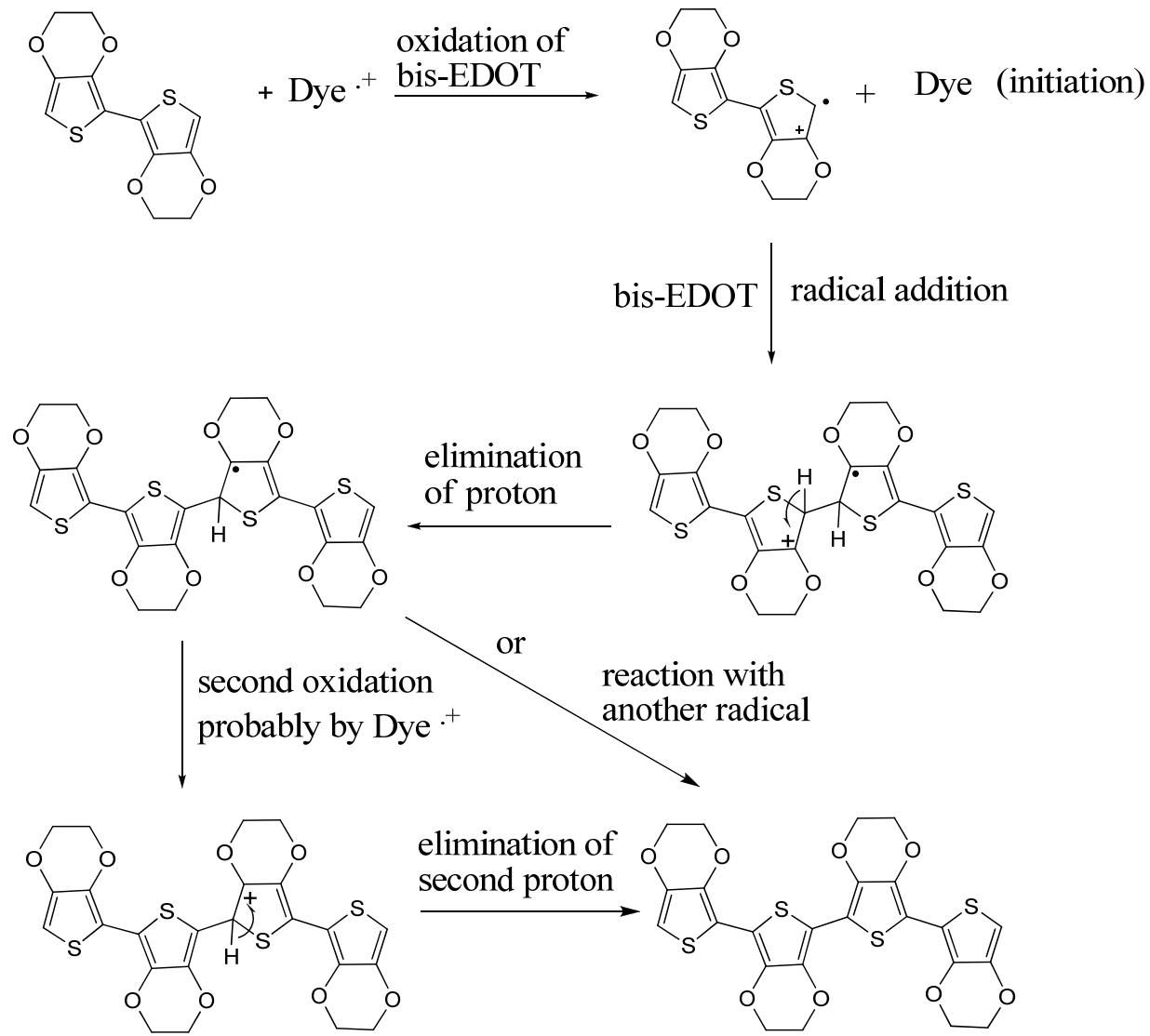

4. Repeat of steps in 3, for oligomer

Overall reaction: 2 bis-EDOT $\longrightarrow(\text { bis-EDOT) })_{2}+2 \mathrm{H}^{+}+2 \mathrm{e}^{-}$

Figure 3.3: Proposed mechanism of polymerization of bis-EDOT to PEDOT by PEP method.

The formation of discontinuities in conjugation (and therefore conductivity) in PEDOT formed by polymerization of bis-EDOT is shown in Figures 3.4-3.4c. The radical cation (1) (Figure 3.4) formed by addition of a bis-EDOT radical cation to a 
molecule of bis-EDOT will deprotonate to form the neutral, stabilised radical 2 (Figure 3.4).

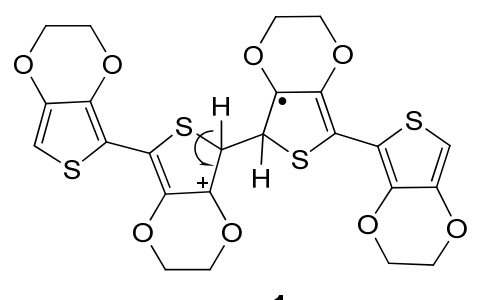

1

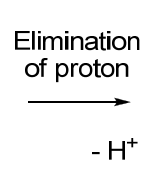

Figure: 3.4

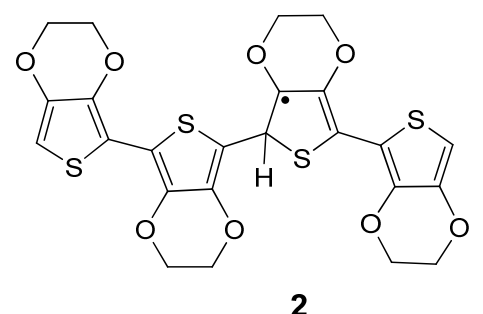

2

This uncharged product (2) has another resonance structure in which the unpaired electron is localised on carbon 1 of the terminal thiophenyl group. This species can then undergo an addition reaction with another molecule of bis-EDOT to form another intermediate radical (3) (Figure 3.4a), with 6 thiophenyl rings.
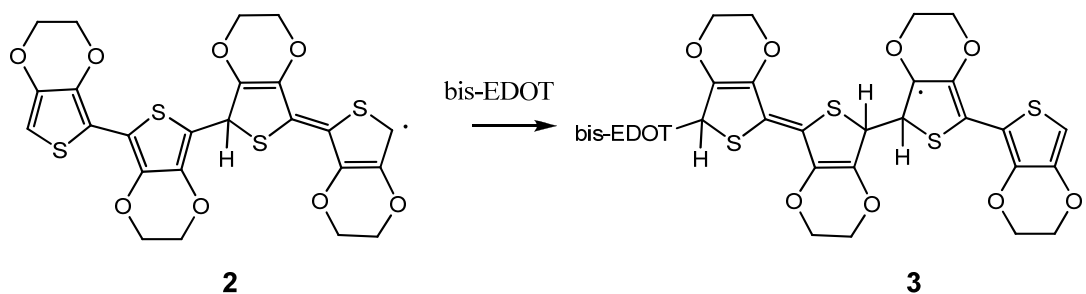

Figure: 3.4a

This new radical 3 can then undergo a one electron oxidation by contact with either the oxidised dye, or with the bis-EDOT radical cation. A stabilised carbocation (4) (Figure 3.4b) results.

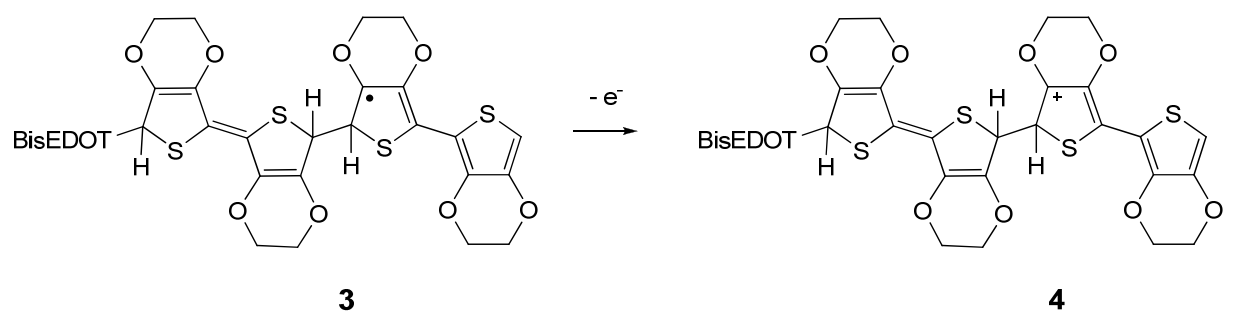

Figure: 3.4b 
Carbocation 4 (Figure 3.4b) can then can eliminate a proton, forming a neutral product (5) (Figure 3.4c) in which the double bonds are no longer fully conjugated.

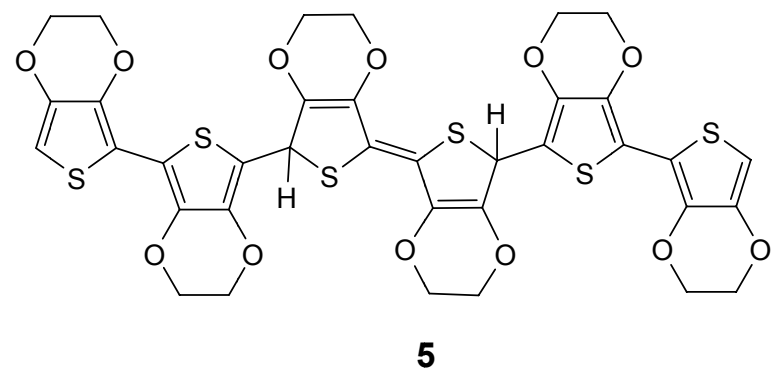

Figure: 3.4c

The 'dihydro' unit in the middle acts as an electrical insulator. The formation of these types of structures can account for the sudden drop off in cell performance.

The same conditions were used for photo-electrochemical polymerization of tris-

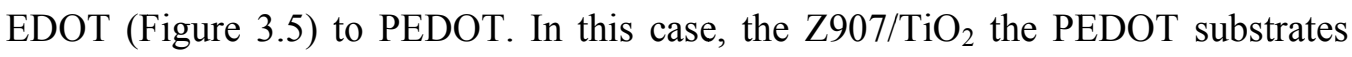
were immersed in a solution of $0.10 \mathrm{M} \mathrm{LiClO}_{4}$ and $0.01 \mathrm{M}$ tris-EDOT in acetonitrile.

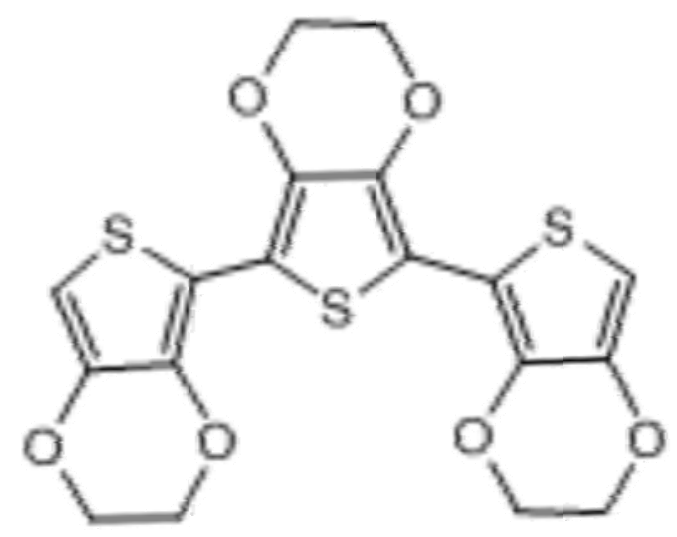

Figure 3.5: Oligomer tris-EDOT used as a HTM for ssDSCs. 


\subsubsection{Ionic liquid treatment}

After photo electrochemical polymerization, the resultant $\mathrm{PEDOT} / \mathrm{Z} 907 / \mathrm{TiO}_{2}$ films were rinsed with ethanol (to remove excess PEDOT on the surface) and treated with a solution of the ionic liquid (1-ethyl-3-methylimidazolium bistrifluoromethanesulfonamide (EMITFSI) containing a 0.20 M lithium bistrifluoromethanesulfonamide (LiTFSI) and 0.2M 4-tert-butylpyridine for $24 \mathrm{hrs}$.

\subsubsection{Assembly of PEDOT-based solid-state DSCs}

Finally, sandwich-type solid-state DSCs were fabricated by clipping mechanically together the counter electrode (Goretex ${ }^{\circledR}$-Au-PEDOT) and the photo-anode $\left(\mathrm{PEDOT} / \mathrm{Z} 907 / \mathrm{TiO}_{2} / \mathrm{FTO}\right)$. The whole cell structure of solid-state dye-sensitized solar cells with HTM has been shown in section 2.3 (Chapter 2).

\subsubsection{Fabrication of liquid dye-sensitized solar cells (DSCs)}

The detailed procedure has already been described in section 2.4.7 (Chapter 2).

Liquid DSCs were fabricated to compare with the solid-state DSCs produced in this Chapter. The following fabrication steps for the DSCs were employed:

i. $\quad$ Prepare mesoporous $\mathrm{TiO}_{2}$ film layer by doctor-blading.

ii. Sintering $\mathrm{TiO}_{2}$ film in muffle furnace.

iii. Dye sensitisation of the $\mathrm{TiO}_{2}$ film.

iv. Device fabrication and testing.

\subsubsection{Total polymerization charge}

The total polymerization charge was determined by integrating the photo-current over time.The total polymerization charge $(\mathrm{Q})=\int_{0}^{t} I d t$, Where as $I=$ photo-current and $t=$ growth time. 


\section{3 Results and discussion}

\subsubsection{Calculation of HOMO-LUMO energy levels and band gaps}

The purpose of calculations of HUMO-LUMO level of the dyes was:

i. To evaluate the suitability of commercial Solaronix ruthenium-based dyes for the photo-electrochemical deposition of PEDOT for solid-state solar cells.

ii. To establish reliable procedure for rapid screening of suitable sensitizing dyes/monomers for photo-electrochemical PEDOT deposition.

Cyclic-voltammograms (CVs) ruthenium-based $\mathrm{Z} 907$ dye, and dye sensitised $\mathrm{TiO}_{2}$ film along with standard ferrocene, have been performed to determine their onset oxidation potential and with the aid of UV-Vis spectra studies to calculate their HOMO and LUMO energy levels.

\subsubsection{Cyclic voltammetry}

Cyclic voltammogram for Z907 dye solution was recorded as described in the experimental [section 3.2.1.1]. Typical of the CVs obtained was that of the Z907 dye solution and shown in Figure 3.6. The onset oxidation potential was determined by a graphical method (shown in Figure 3.8). In general, the potential at which the lines determined by the baseline and the rising slope of the first cycle intersect was taken as the onset oxidation potential (Figure 3.8). The oxidation potential thus calculated for dye Z907 solution is $0.318 \mathrm{~V}$ (vs. $\mathrm{Ag} / \mathrm{Ag}+$ ). 


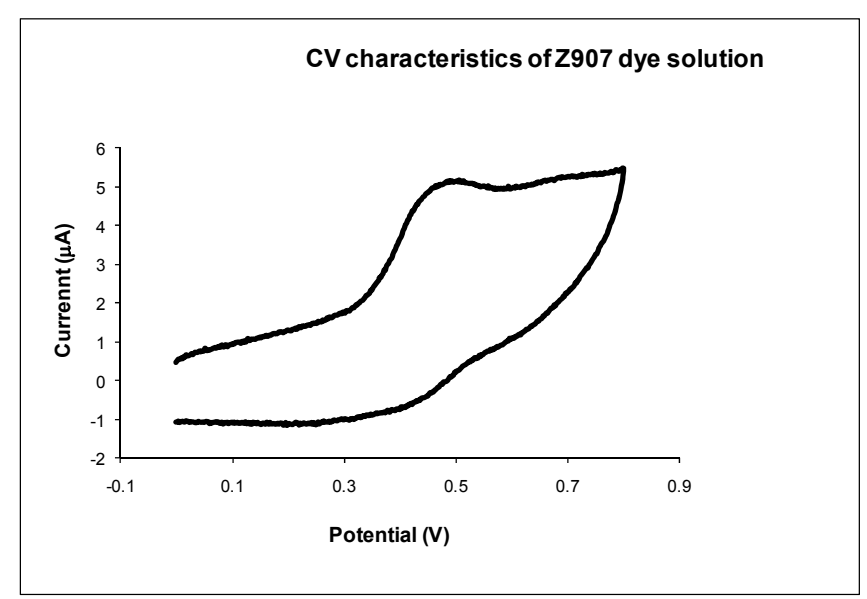

Figure 3.6: $\mathrm{CV}$ of Z907 dye solution (1 cycle); electrolyte: $0.10 \mathrm{M}$ TBAP in acetonitrile; upper potential limit $=+1000 \mathrm{mV}$, lower potential limit $=$ $0.0 \mathrm{mV}$, scan rate $=100 \mathrm{mVs}^{-1}$; glassy carbon working electrode, $\mathrm{Ag} /$ $\mathrm{Ag}^{+}$reference electrode and $\mathrm{Pt}$ mesh auxiliary electrode.

The $\mathrm{CV}$ of $\mathrm{Z} 907$ dye sensitized $\mathrm{TiO}_{2}$ was also recorded. The $\mathrm{CV}$ of $\mathrm{Z} 907$ dye sensitized $\mathrm{TiO}_{2}$ film is shown in Figure 3.7. The onset oxidation potential was determined by the same graphical method as above. The oxidation potential of the Z907 dyes on $\mathrm{TiO}_{2}$ was found to be $0.337 \mathrm{~V}$ (vs. Ag/ Ag+). Oxidation potentials were more positive on $\mathrm{TiO}_{2}$ compared to the oxidation potentials observed earlier for the free dyes in solution. The oxidation potential for $\mathrm{Z907}$ dye on $\mathrm{TiO}_{2}$ was more positive than that in the solution because the bond formed between the $\mathrm{TiO}_{2}$ surface and the $-\mathrm{COOH}$ and hence increased the electron withdrawing character of the ligand (which is already present in the dye molecule) and therefore it increased the oxidation potential of the $\mathrm{Ru}^{2+} / \mathrm{Ru}^{+3}$ which overall shifted towards more positive potential $[7 \mathrm{a}, 7 \mathrm{~b}]$. It has also been reported (1-4) earlier that reorganization energy is needed for any molecule solvated on the surface as compared in solution i.e. HOMOLUMO energy level is stabilized by solvent molecule in solution[7c, 7d]. The oxidation potential of dye in solution is less because the potential is solvent stabilized. 


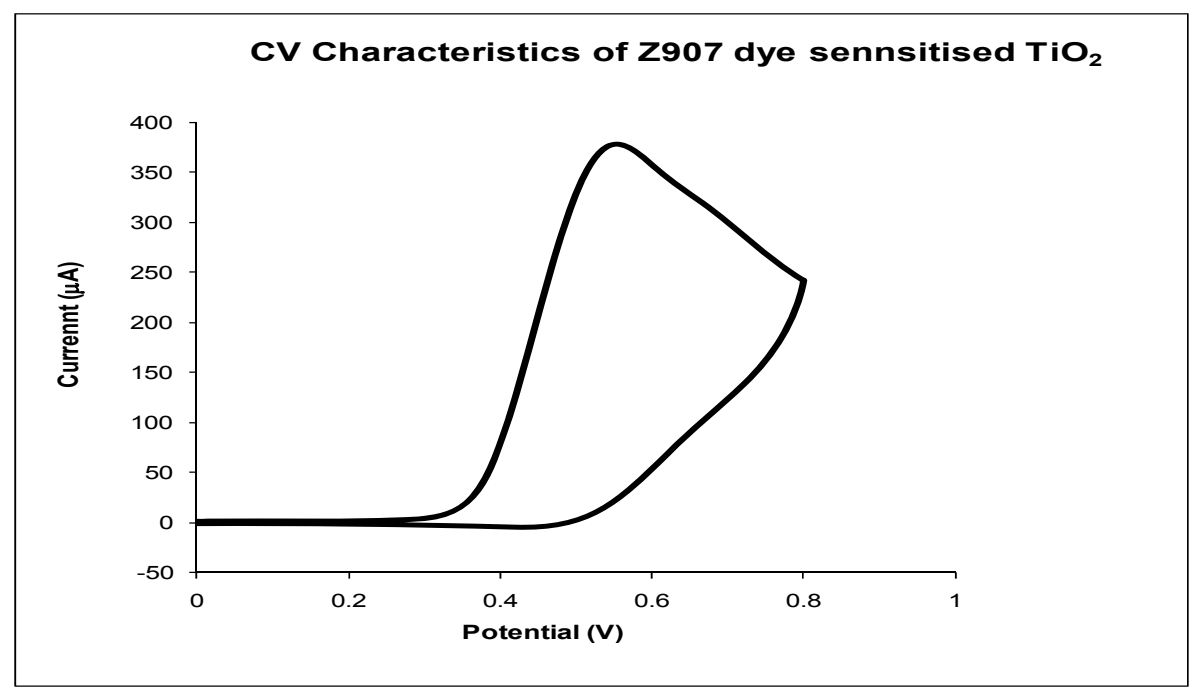

Figure 3.7: $\mathrm{CV}$ of a $\mathrm{Z} 907$ dye sensitized $\mathrm{TiO}_{2}$ film (3 cycles). Electrolyte: $0.10 \mathrm{M}$ TBAP in acetonitrile $(\mathrm{ACN})$; upper potential limit $=+800 \mathrm{mV}$, lower potential limit $=0.0 \mathrm{mV}$, scan rate $=100 \mathrm{mVs}^{-1}$; dyed $\mathrm{TiO}_{2}$ working electrode, $\mathrm{Ag} / \mathrm{Ag}^{+}$reference electrode and Pt mesh auxiliary electrode.

In Figure 3.8 the $\mathrm{CV}$ of ferrocene is presented. The organometallic sandwich type compound ferrocene, $\mathrm{Fe}\left(\mathrm{C}_{2} \mathrm{H}_{5}\right)_{2}$, is used here as an internal standard for calibrating redox potentials in non-aqueous solvent. Ferrocene undergoes a one electron oxidation at a low potential of $\sim 0.5 \mathrm{~V}$ vs. saturated calomel electrode (SCE), and is also used as a standard in electrochemistry as $\mathrm{Fc}^{+} / \mathrm{Fc}=0.64 \mathrm{~V}$ vs. normal hydrogen electrode (NHE).

A typical cyclic voltammogram of ferrocene is shown in Figure 3.8. Two peaks are observed associated with the reversible single electron transfer. The peak on the positive (forward) scan is due to oxidation of neutral ferrocene to a positively charged $(\mathrm{C} 2 \mathrm{H} 5)_{2} \mathrm{Fe}^{+}$state, and the peak on the negative (reverse) scan is due to reduction back to the neutral state. The total charge passed during the redox process can be estimated by measuring the area under the curve. The oxidation and reduction potentials of the $\mathrm{Fc} / \mathrm{Fc}^{+}$couple were calculated as shown in Figure 3.8. 


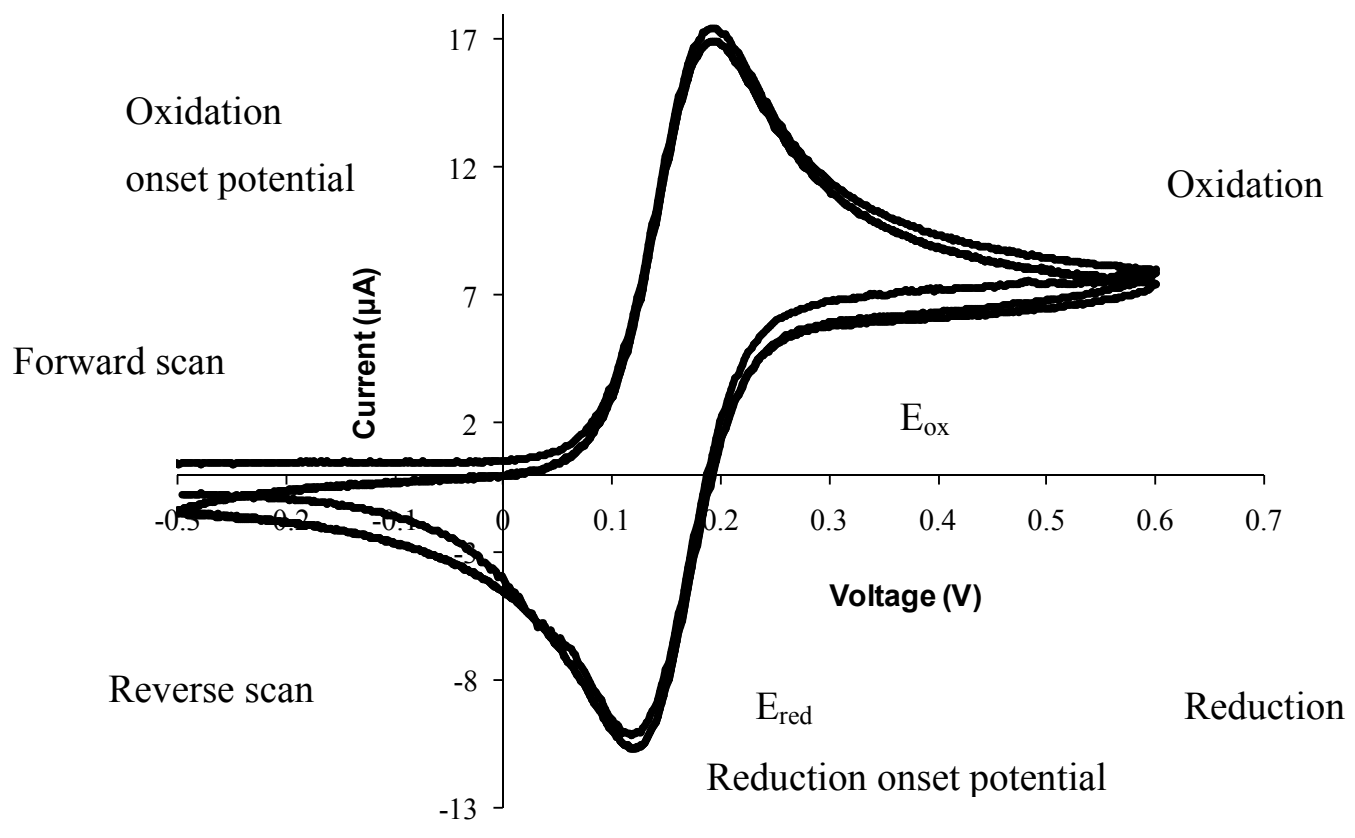

Figure 3.8: $\mathrm{CV}$ of ferrocene standard (3 cycles), demonstrating a single reversible electron transfer process on a glassy carbon electrode working electrode $\left(\mathrm{SA}=1.8 \mathrm{~mm}^{2}\right)$. Electrolyte: $0.10 \mathrm{M}$ TBAP in $\mathrm{ACN}$; upper potential limit $=+600 \mathrm{mV}$, lower potential limit $=-300 \mathrm{mV}$, scan rate $=100 \mathrm{mVs}^{-1} ; \mathrm{Ag} / \mathrm{Ag}^{+}$reference electrode and Pt mesh auxiliary electrode.

\subsubsection{UV-Vis spectral studies}

The UV-Vis spectra of ruthenium-based Z907 dye and of associated dye-sensitized $\mathrm{TiO}_{2}$ films were recorded. For example, the UV-Vis absorption spectrum of Z907 in ethanol solution is shown in Figure 3.9. The optical band gap was determined using a graphical method. The determined optical band gap was $2.07 \mathrm{eV}$ in ethanol solution. In the corresponding $\mathrm{Z} 907$ dye sensitized $\mathrm{TiO}_{2}$ films, the determined optical band gaps were also found $2.07 \mathrm{eV}$. The band gap was determined from the absorption edge of the Z907 dye [7a]. Absorption edge band is the point on an absorption spectrum which is the mimimum energy needed from the valence band to conduction band. 
To determine the band gap, following steps have to be followed:

i. Drawn the line which best fits the slope of the absorption edge of dye.

ii. Drawn another line that extrapolated the background line before the absorption edge is formed.

iii. The point of intersection of the two lines is band gap. This value found in $\mathrm{nm}$ and converted to $\mathrm{eV}$.

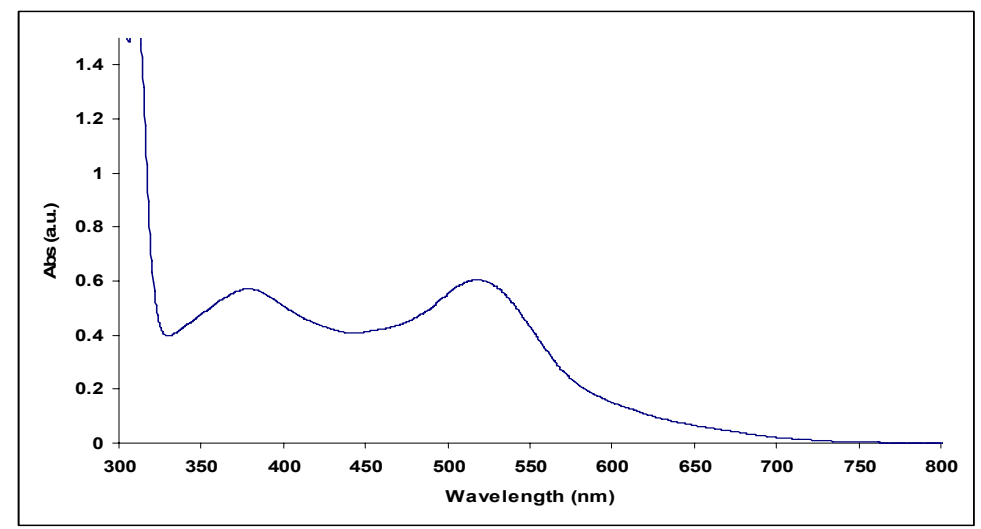

Figure 3.9: Determination of $\lambda$ onset from UV-Vis absorption spectrum of a $5 \times 10^{-5}$ M solution of ruthenium dye (Z907) in ethanol.

The redox potential of dyes in solution and dyes attached to $\mathrm{TiO}_{2}$ films was measured by cyclic voltammetry and band gap was calculated by UV-Vis absorption spectroscopy.

In general, the energy of the HOMO, LUMO and band gap of dye and dyesensitized $\mathrm{TiO}_{2}$ film material were determined as follows:

Band gap $(\mathrm{eV})=1243 / \lambda$ (onset) $\mathrm{nm}$; where $1243=h c / 1.6 \times 10^{-19}$, Where $h=$ Planck's constant $\left(6.62 \times 10^{-34} \mathrm{Js}\right), c=$ velocity of light $\left(3.00 \times 10^{8} \mathrm{~m} / \mathrm{s}\right)$.

So, band gap of Z907 dye $=1243 / 590=2.10 \mathrm{eV}, \lambda$ (onset) $=590 \mathrm{~nm}$ (From Figure 3.9).

The HOMO and LUMO values were calculated as reported earlier [8]. The HOMO and LUMO values were transformed via the equation $E(L U M O / H O M O)-e(4.88+$ $\left.V_{\text {redox }}\right)$, [8] where $V_{\text {redox }}$ is the onset potential vs. ferrocene of reduction or oxidization of sensitizers dissolved in DMF. 
The energy levels of HOMO determined from the onset oxidation potential $\left(\mathrm{E}^{\mathrm{ox}}\right)$ and estimated on the basis of reference energy level of ferrocene $(4.8 \mathrm{~V}$ below the vaccum level). Here HOMO/ LUMO calculated as the procedure given by Wang et al. [8].

Ionisation potential (I.P.) / HOMO (Highest Occupied Molecular Orbital).

$\underline{1 .}$ HOMO of $\mathrm{Z907}$ dye attached to $\mathrm{TiO}_{2}$ film:

Calculated from CV (Figure 3.5)

$=-\mathrm{e}\left[\mathrm{E}^{\mathrm{ox}}{ }_{\text {onset }}-\mathrm{E}_{\text {ferrocene }}{ }^{1 / 2}+4.8\right]$

$=-\mathrm{e}[0.337-0.158+4.8]$

$=-4.97 \mathrm{eV}$ (vs. vaccum)

This value is equal to that obtained previously by Wang et al. [8].

Lowest Unoccupied Molecular Orbital (LUMO) will be calculated as described in [8]

$$
\text { So, } \begin{aligned}
\text { LUMO } & =E_{g(e V)}{ }^{{ }^{o p t}}+\text { HOMO } \\
& =2.10 \mathrm{eV}+(-4.97 \mathrm{eV}) \\
& =-2.87 \mathrm{eV} .
\end{aligned}
$$

The $-2.90 \mathrm{eV}$ LUMO value relative to the conduction band edge $(-4.00 \mathrm{eV}$ vs. vacuum) of $\mathrm{TiO}_{2}$ [8] provides a thermodynamic driving force for charge generation.

Similarly, the HOMO and LUMO of Z907 dye in solution was calculated as above.

$=-\mathrm{e}\left[\mathrm{E}_{\text {onset }}^{\text {ox }}-\mathrm{E}_{\text {ferrocene }}{ }^{1 / 2}+4.8\right]$

$=-\mathrm{e}[0.318-0.158+4.8]$

$=-4.96 \mathrm{eV}$ (vs. vaccum)

And $\mathrm{LUMO}=\mathrm{E}_{\mathrm{g}(\mathrm{eV})}{ }^{\mathrm{opt}}+\mathrm{HOMO}=-2.86 \mathrm{eV}$ (vs. vacuum).

It is observed that the determined values of HOMO and LUMO energy levels of dye solution and dye attached into $\mathrm{TiO}_{2}$ film are almost equal. Roncali et al. reported [9] that HOMO of bis-EDOT is $-4.84 \mathrm{eV}$. The HOMO of Z907 dye experimentally calculated here is $-4.97 \mathrm{eV}$; therefore this dye could be used as a potential sensitizer in situ photo-electrochemical polymerization for the preparation of photo anode. It 
has been proposed that the photo-electrochemical polymerization could be possible only if the HOMO of dye will be higher than the HOMO of HTM.

\subsubsection{Dye uptake by $\mathrm{TiO}_{2}$}

Molar extinction coefficients were calculated using the procedure below:

$3 \mu \mathrm{m}$ thick $\mathrm{TiO}_{2}$ films were sintered for $2 \mathrm{hrs}$ to remove water, and then immersed immediately in solution of the dye (Z907) in tert-butanol/acetonitrile (1/1) dye for 24 hrs. UV-Vis spectra were then recorded to investigate the dye loading of Z907 dye on the $\mathrm{TiO}_{2}$ films.

These loading measurements were corroborated by also measuring the concentration of dye that could be desorbed from the same films, as follows: sintered $\mathrm{TiO}_{2}$ films were immersed in the Z907 dye solution for $24 \mathrm{hrs}$. UV-Vis spectra of these sensitized films were recorded. The films were then immersed in a $10 \mathrm{ml}$ solution of $0.10 \mathrm{M} \mathrm{NaOH}$ in $1: 1$ of $\mathrm{C}_{2} \mathrm{H}_{5} \mathrm{OH}: \mathrm{H}_{2} \mathrm{O}$ to desorb the dyes from the respective films. The UV-Vis spectrum of each desorption solution was recorded.

Found Z907 dye $\lambda_{\max }=513 \mathrm{~nm}$,

Using Beers Law, $\mathrm{A}=\varepsilon l c(l=1 \mathrm{~cm})$, the extinction coefficient $(\varepsilon)$ is given by $\varepsilon=\mathrm{A} / c$.

For Z907 at 513nm, Abs $=0.604$ (Figure 3.10),

Therefore $\varepsilon$ Z907, $513 \mathrm{~nm}=0.604 / 5 \times 10^{-5}=12,080 \mathrm{~mole}^{-1} \mathrm{~cm}^{-1}$

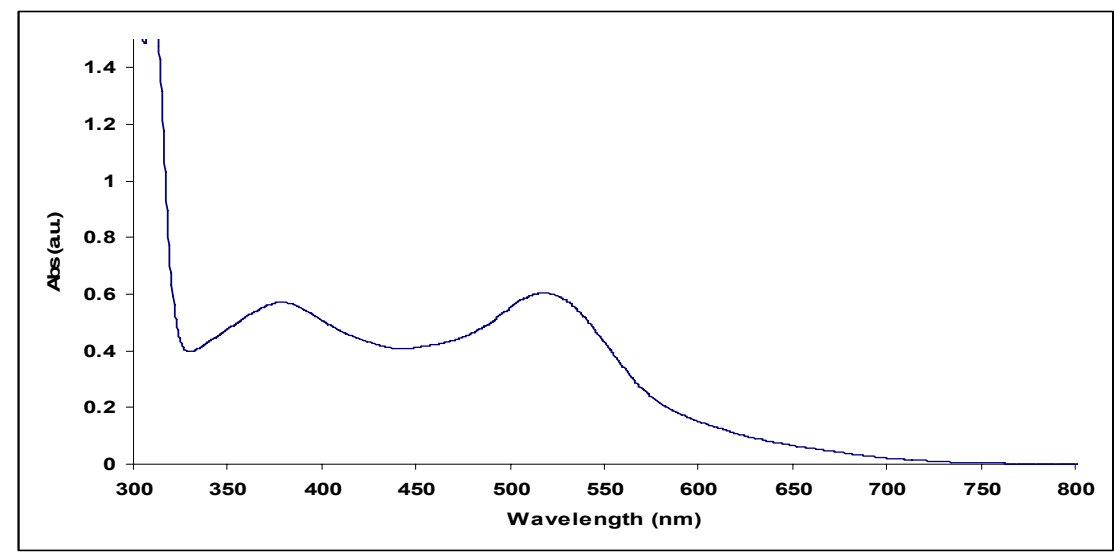

Figure 3.10: UV-Vis absorption spectrum for a $5 \times 10^{-5} \mathrm{M}$ solution of ruthenium dye Z907 in ethanol. 


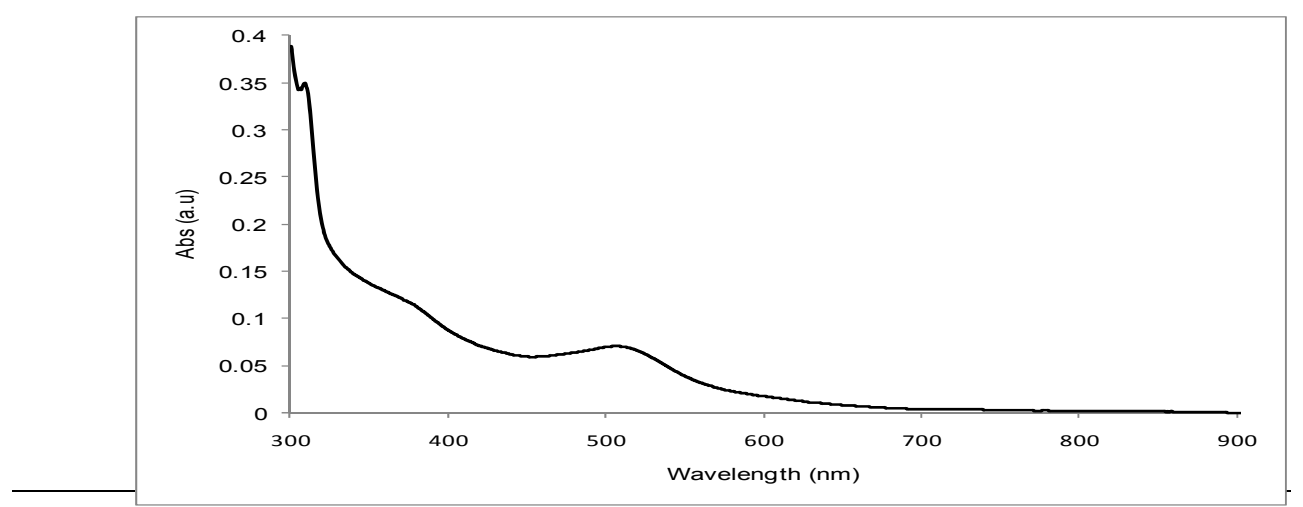

Figure 3.11: UV-Vis absorption spectrum for dye desorbed from a thin $(3 \mu \mathrm{m}) \mathrm{TiO}_{2}$ film sensitized with the ruthenium based Z907 dye.

Calculation of surface coverage of Z907 dye:

From UV-Vis spctra of Z907 dye desorption solution, Z907 dye Abs. at $513 \mathrm{~nm}=$ 0.070 (Figure 3.11)

Surface coverage of Z907 dye is calculated as follows;

As we know, $\mathrm{A}=\varepsilon c$, so $c=\mathrm{A} / \varepsilon=0.070 / 12,080$

$c_{\text {Z907 }}=5.8 \times 10^{-6}$ moles $/ \mathrm{L}$

Therefore, number of moles of dye; $\eta_{\mathrm{Z} 907}=c_{\mathrm{z} 907} \times \mathrm{V}_{\text {desorption }}$

$$
\begin{aligned}
= & 5.8 \times 10^{-6}(\text { moles } / \mathrm{L}) \times 0.01 \mathrm{~L} \\
& =5.8 \times 10^{-8} \text { moles }
\end{aligned}
$$

Hence, Surface coverage $\Gamma=\eta /$ Area $\times$ thickness of $\mathrm{TiO}_{2}$ film

$$
\begin{aligned}
\Gamma_{\text {z } 907} & =5.8 \times 10^{-8} \text { moles } /\left(0.765 \mathrm{~cm}^{2} \times 3 \mu \mathrm{m}\right) \\
& =2.5 \times 10^{-8} \text { moles } \mathrm{cm}^{-2} \mu \mathrm{m}^{-1} .
\end{aligned}
$$

Note: The thickness of the $\mathrm{TiO}_{2}$ film was $3 \mu \mathrm{m}$ and area of the film from where the dye was desorbed was $8.5 \mathrm{~mm} \times 9 \mathrm{~mm}$ (i.e. $0.765 \mathrm{~cm}^{2}$ ), and the volume into which the desorbed dye was dissolved $10 \mathrm{ml}$. UV-Vis absorption spectrum for dye desorbed from a thin $(3 \mu \mathrm{m}) \mathrm{TiO}_{2}$ film sensitized with the ruthenium based $\mathrm{Z907}$ dye is presented in Figure 3.11.

The HOMO/ LUMO values of various ruthenium and non-ruthenium based dyes along with onset oxidation potential of oligomers are presented in Tables 3.1 and 3.2 respectively. 
Table 3.1: Comparison of energy level data determined here for various dyes with literature values.

\begin{tabular}{|c|c|c|c|c|c|}
\hline Dye & $\begin{array}{c}\text { HOMO } \\
(\mathrm{eV}) \\
\text { vs. } \\
\text { vaccum }\end{array}$ & $\begin{array}{c}\text { LUMO } \\
(\mathrm{eV}) \\
\text { vs. } \\
\text { vaccum }\end{array}$ & $\begin{array}{c}\text { Band gap } \\
(\mathrm{eV})\end{array}$ & $\begin{array}{c}\text { Molar extinction } \\
\text { coefficient }\left(\mathrm{M}^{-1} \mathrm{~cm}^{-1}\right) \\
\text { Determined here }\end{array}$ & $\begin{array}{l}\text { Molar extinction coefficient }\left(\mathrm{M}^{-}\right. \\
\left.{ }^{1} \mathrm{~cm}^{-1}\right) \text { Literature value }\end{array}$ \\
\hline $\begin{array}{l}\text { Z907 } \\
\text { Dye }\end{array}$ & -4.99 & -3.35 & 1.64 & $\begin{array}{c}12080 \\
\text { @ } 513 \mathrm{~nm}\end{array}$ & $\begin{array}{c}12536 \\
\text { @ } 513 \mathrm{~nm}\end{array}$ \\
\hline $\begin{array}{l}\text { N719 } \\
\text { Dye }\end{array}$ & -5.18 & -3.19 & 1.99 & N.A & $\begin{array}{c}14000 \\
@ 533 \mathrm{~nm}\end{array}$ \\
\hline N3 dye & -5.34 & -3.30 & 2.04 & N.A & $\begin{array}{l}1.4 \times 10^{4} \\
\text { @ } 532 \mathrm{~nm}\end{array}$ \\
\hline $\begin{array}{l}\text { GD2 dye } \\
\text { b }\end{array}$ & -5.31 & -3.29 & 1.89 & $\begin{array}{c}122600 \\
\text { (a) } 436 \mathrm{~nm}\end{array}$ & $\begin{array}{c}140000 \\
\text { @436 nm }\end{array}$ \\
\hline $\begin{array}{l}\text { P-257 } \\
\text { Dye }^{\mathbf{b}}\end{array}$ & -5.61 & -3.42 & 2.51 & $\begin{array}{c}24056 \\
@ 416 \mathrm{~nm}\end{array}$ & 25000 \\
\hline $\begin{array}{l}\text { D-149 } \\
\text { Dye }^{\mathbf{b}}\end{array}$ & -5.03 & -2.63 & 2.43 & $\begin{array}{c}68700 \\
\text { @ } 526 \mathrm{~nm}\end{array}$ & N.A \\
\hline
\end{tabular}

Note: a. Z907 Dye: Nano Lett, 6 (4) (2006) 769-773, N3 Dye: J. Phy. Chem. B, 107 (2003) 597-606, N719 Dye: Chem. Comm. 46 (2007) 4887-4889, GD2 Dye: : Chem. Comm., 39 (2008) 4741-4743, P-257 Dye: Chem. Mater., 16, 2004, 1806-1812, D-149: Angew Chem Int. Ed, 47, 2008, 1923-1927. b. See Chapter 6.

Table 3.2: Literature electrochemical and optical data for EDOT and its oligomers.

\begin{tabular}{|c|c|c|c|c|c|c|}
\hline $\begin{array}{c}\text { Name of } \\
\text { polymer }\end{array}$ & $\begin{array}{c}E_{p, n}^{\text {ox }} \\
(\mathrm{V})\end{array}$ & $E_{\text {polymer }(\mathrm{V})}^{\text {ox }}$ & $E_{\text {polymer }(\mathrm{V})}^{\text {red }}$ & $\begin{array}{c}\lambda_{\max } \\
(\mathrm{nm})\end{array}$ & $\begin{array}{c}E_{g} \\
(\mathrm{eV})\end{array}$ & References \\
\hline EDOT & $\sim 1.1$ & $-0.14 / 0.16$ & $-0.37 / 0.21$ & 499 & 2.05 & $\begin{array}{c}\text { Adv.Mater., } 15 \\
(2003) 855-879\end{array}$ \\
\hline bis-EDOT & 0.56 & $-0.25 / 0.10$ & $-0.30 /-0.83$ & 566 & 1.65 & Ditto \\
\hline ter-EDOT & 0.20 & -0.05 & $-0.55 / 0.85$ & 495 & 1.60 & Ditto \\
\hline
\end{tabular}

Note: EDOT oxidation potential approx. 1.0V (vs. $\mathrm{Ag} / \mathrm{Ag}^{+}$). 2. bis-EDOT oxidation potential approx. $0.4 \mathrm{~V}$ (vs. $\left.\mathrm{Ag} / \mathrm{Ag}^{+}\right)$. 


\subsubsection{Photo-polymerization of EDOT}

The total charge transferred during polymerization was calculated by integration of the polymerization current. The total polymerization charge employed in deposition of PEDOT in dyed $\mathrm{TiO}_{2}$ layers depends on the light intensity, duration of polymerization, thickness of $\mathrm{TiO}_{2}$, dopant, media, and different oligomers (e.g. bisEDOT and tris-EDOT).

\subsubsection{Light intensity}

Different light intensities for the photo-electrochemical polymerization were also examined and their total polymerization charge was calculated. The light intensity was varied during photo-electrochemical polymerisation by using different optical neutral density filters. The light under which photo-electrochemical polymerisation was carried out was $5,17,20,21,22,30,50,75,89,100$, and $200 \mathrm{~mW} / \mathrm{cm}^{2}$. The highest polymerization charge was measured $18 \mathrm{mC} \mathrm{cm}^{-2}$, and this achieved by potentiostatic polymerization for 30 minutes when the light intensity of the light was $89 \mathrm{~mW} / \mathrm{cm}^{2}$. For the photovoltaic performance of ssDSCs, the kinetic of the graph should be like with photo-polymerization done with light intensity of $22 \mathrm{~mW} / \mathrm{cm}^{2}$. When light intensity was $22 \mathrm{~mW} / \mathrm{cm}^{2}$, firstly, the current under constant illumination increased and reached its maximum after $600 \mathrm{sec}$, and then it decreased (Figure 3.12). For experiments using different light intensities, it was observed that more charge was passed during polymerization when irradiated with light of 74 and 89 $\mathrm{mW} / \mathrm{cm}^{2}$ intensities. Above $22 \mathrm{~mW} / \mathrm{cm}^{2}$ light intensity, the total polymerization charge was higher and below $22 \mathrm{~mW} / \mathrm{cm}^{2}$ light intensity, the total polymerization charge was smaller. The most efficient photo-anode was prepared when the growth time of PEDOT was 30 minutes and the light intensity during photo-electrochemical polymerization (PEP) was $22 \mathrm{~mW} / \mathrm{cm}^{2}$ and in acetonitrile solvent. The efficiency of ssDSCs depended on how the PEDOT grew into dyed $\mathrm{TiO}_{2}$ matrix rather than the quantity of charge developed into the dyed $\mathrm{TiO}_{2}$. In Figure 3.12, the integrated photo polymerization charge shown along with the photo-current during photoelectrochemical polymerization. 


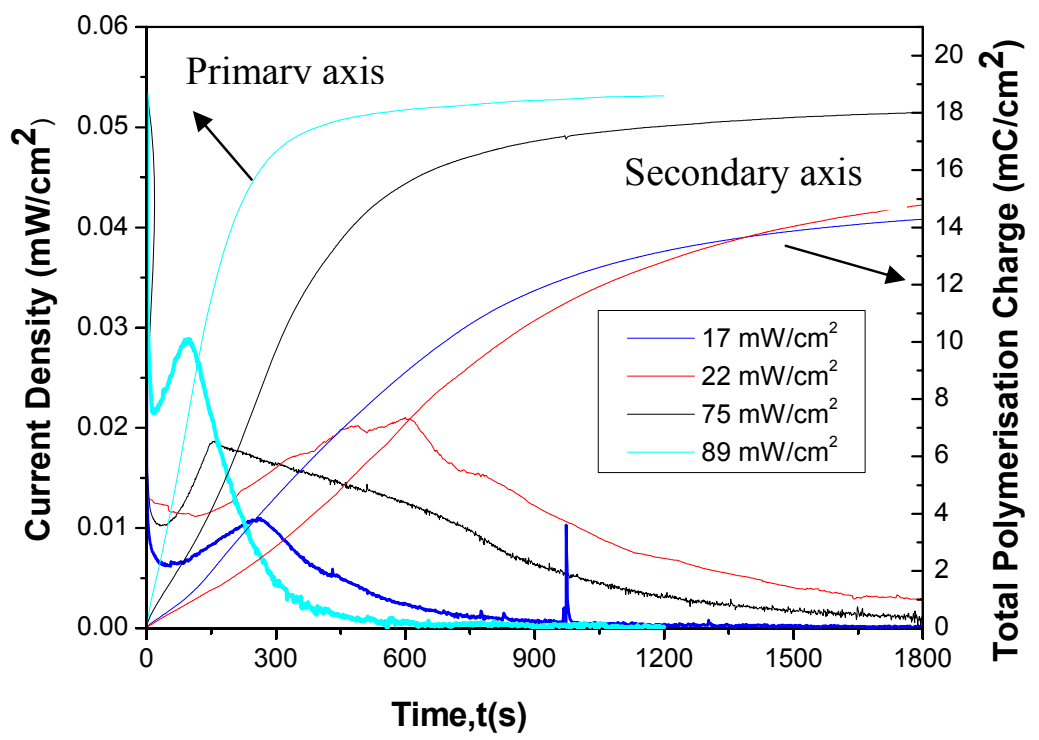

Figure 3.12: Total polymerization charge (secondary axis) calculated when different intensity of light applied during photo-electrochemical polymerization, while the photo-current is shown in primary axis.

\subsubsection{PEDOT growth time}

The total polymerization charge was calculated in an experiment in which a range of PEDOT growth times were varied during photo-electrochemical polymerization. It is observed from Figure 3.13 that when PEDOT was grown for 30 minutes in acetonitrile, nearly $15 \mathrm{mC} / \mathrm{cm}^{2}$ was achieved and the charge increased with increase of PEDOT growth time.

It has been reported earlier [10] that all charges passed through the circuit during photo-electrochemical polymerization was fully utilised for the oxidative coupling of bis-EDOT. The dye to PEDOT ratio was roughly 1.2 [10] when the PEDOT was grown for 30 minutes. This ratio decreased if the amount of PEDOT was decreased [10]. It has been reported in our JACS paper [10] that each bis-EDOT unit needs one electron to oxidise to PEDOT. 


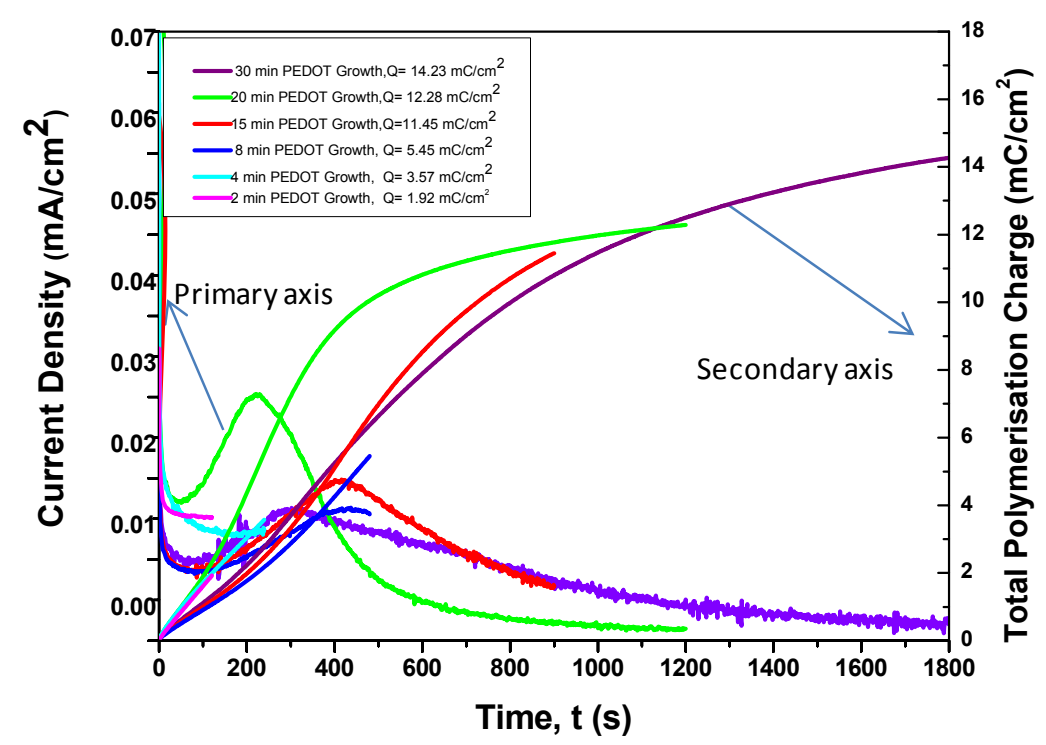

Figure 3.13: Total polymerization charge (secondary axis) calculated when different amounts of PEDOT were grown into $\mathrm{TiO}_{2}$ from bis-EDOT in acetonitrile using $22 \mathrm{~mW} / \mathrm{cm}^{2}$ light intensity, while the photo-current is shown in primary axis.

\subsubsection{Thickness of mesoporous $\mathrm{TiO}_{2}$ layer}

The charge was also dependent on the thickness of the $\mathrm{TiO}_{2}$ of working electrode. Moreover, the morphology of the mesoporous $\mathrm{TiO}_{2}$ film had a great effect on the photo-polymerization. The pore size, effective surface area, porosity and particle size also affected the effective polymerization of bis-EDOT to PEDOT. This will be explained in Chapter 4 in detail. It is found that when the thickness of $\mathrm{TiO}_{2}$ is 5.5 $\mu \mathrm{m}$, more polymerization charge $\left(16.39 \mathrm{mC} / \mathrm{cm}^{2}\right)$ developed where as the charge decreased $\left(13.86\right.$ and $\left.8.77 \mathrm{mC} / \mathrm{cm}^{2}\right)$ when thickness of working electrode either increased $(7 \mu \mathrm{m})$ or decreased $(3 \mu \mathrm{m})$ (see Figure 3.14). It is also observed that 5.5 $\mu \mathrm{m}$ thickness of $\mathrm{TiO}_{2}$ gives better photovoltaic performance of ssDSCs. 


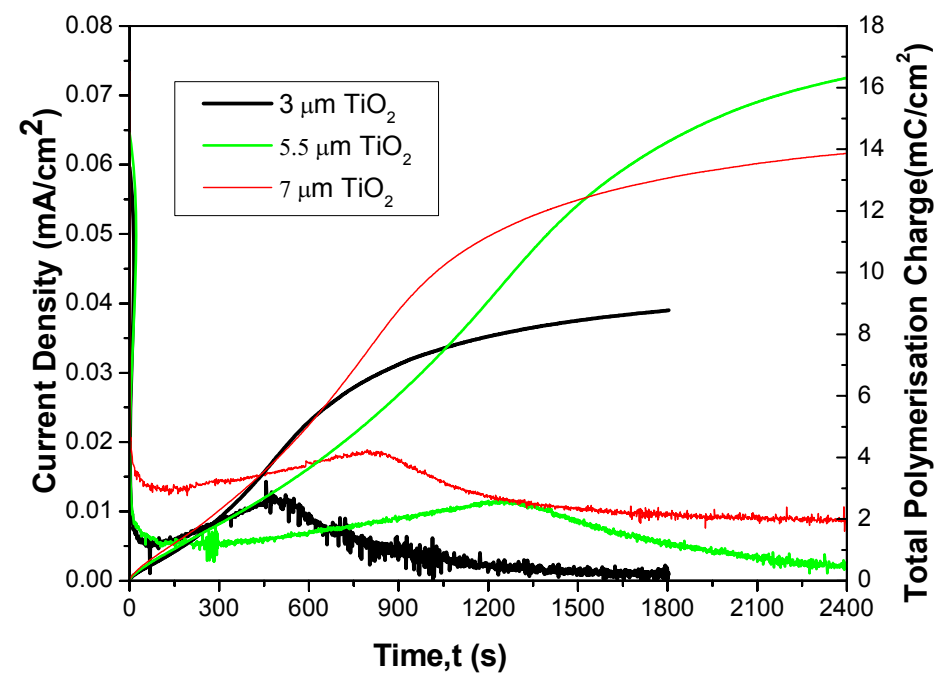

Figure 3.14: Total polymerization charge (secondary axis) calculated when different thicknesses of $\mathrm{TiO}_{2}$ were employed for working electrode during photo-electrochemical polymerization, while the photo-current is shown in primary axis.

\subsubsection{PEDOT growth in ionic liquid}

Ionic liquid was used as a medium, to grow PEDOT well into dye $\mathrm{TiO}_{2}$ nanomatrix. Ma et al. earlier reported [11] that PEDOT can be grown in ionic liquid which is good for removing water/ moisture during the fabrication of electronic devices and these types of electrodes give higher electrochemical stability in electrochromic devices. Ahmed et al. [12] has also reported that a hydrophobic media enhances the electrochemical polymerisation deposition of PEDOT uniformity and leads to formation of thick films. The ionic liquid is comprised of ions and charge on the cations and the counter ion over large volume of the molecule which enhances the electrochemical polymerization. Moreover, ionic liquid has great impact on the chemical and physical properties because the solvent molecule which is present in ionic liquid comprises of charge and the ions on the cation and the counter ion and these are distributed throughout the large molecule by its resonance structure [12]; therefore it can be used as a media for in situ photo-electrochemical polymerization. 
When photo-polymerization was carried out in the ionic liquid (EMITFSI) instead of acetonitrile, more PEDOT was deposited on the dyed $\mathrm{TiO}_{2}$ surface and the product appeared deep dark blue. Images of photo-anodes which were prepared under different conditions are given in Figure 3.16. The photo-current (Figure 3.15) shows how the ionic liquid influences PEDOT deposition. In the ionic liquid, it was possible to grow PEDOT for a longer time in the same light intensity $\left(22 \mathrm{~mW} / \mathrm{cm}^{2}\right)$ than could be done in acetonitrile medium. The total polymerization charge calculated for 45 minutes PEDOT deposition in EMITFSI was $38 \mathrm{mC} / \mathrm{cm}^{2}$ (Figure 3.15) and the PEDOT could also be further grown for longer time (Figure 3.15).

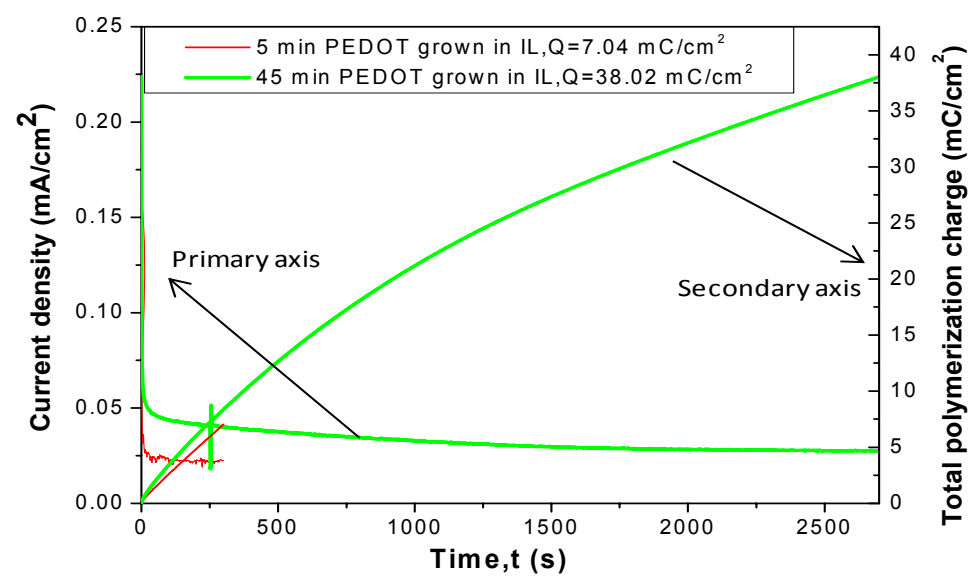

Figure 3.15: Total polymerization charge (secondary axis) calculated when different amounts of PEDOT were grown onto $\mathrm{TiO}_{2}$ from bis-EDOT in thionic liquid (EMITFSI) using $22 \mathrm{~mW} / \mathrm{cm}^{2}$ light intensity, while the photo-current is shown in primary axis.

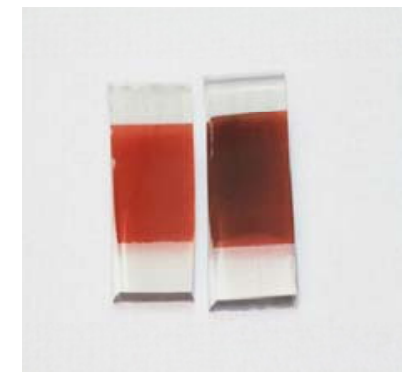

Photo-anode prepared in acetonitrile.

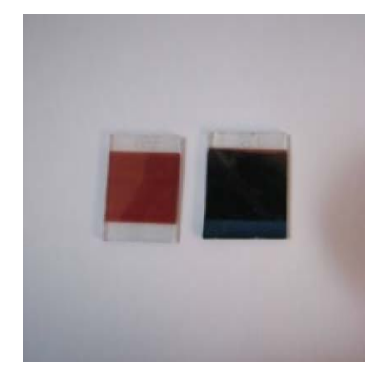

Photo-anode prepared in ionic liquid.

Figure 3.16: The photo-anode prepared using bis-EDOT in acetonitrile (left) and bis-EDOT in ionic liquid (right). The photo-anode before photopolymerization was red and after photo-polymerization became little dark. Note: Experimental conditions; both photo-anodes were prepared under same conditions of light intensity $\left(22 \mathrm{~mW} / \mathrm{cm}^{2}\right)$ and growth time (30 minutes). 


\subsubsection{Different dopant anions}

Yanagida et al. [3] have studied the influence of different doped anions such as $\mathrm{ClO}_{4}{ }^{-}$ ' $\mathrm{CF}_{3} \mathrm{SO}_{3}{ }^{-}, \mathrm{BF}_{4}^{-}$, and $\mathrm{TFSI}^{-}$on PEDOT. They observed TFSI is the best dopant anion which enhanced the hole conductivity of PEDOT and also increased the overall efficiency of ssDSSs. Here different anions (see Figure 3.21) were employed to study the photovoltaic performances of ssDSCs. From Figure 3.17, it is observed that when $\mathrm{CF}_{3} \mathrm{SO}_{3}{ }^{-}$used as dopant anions the photo electrochemical polymerization can be carried out upto $2700 \mathrm{~s}$ (total polymerization charge $=22 \mathrm{mC} / \mathrm{cm}^{2}$ ) and whereas on TFSI leads only $2400 \mathrm{~s}$ (total polymerization charge $=12 \mathrm{mC} / \mathrm{cm}^{2}$ ) and $\mathrm{ClO}_{4}{ }^{-}$(total polymerization charge $=15 \mathrm{mC} / \mathrm{cm}^{2}$ ) only 1800 s. It is reported by Yanagida et al [3] that the conductivities were in the order $\mathrm{BF}_{4}^{-}<\mathrm{ClO}_{4}^{-}<\mathrm{CF}_{3} \mathrm{SO}_{3}^{-}<$TFSI (bistrifluoromethanesulfonylimide) and this was reflected by the photocurrents and the same trend followed for efficiencies of the devices. The higher charge delocalisation on larger TFSI anion compared to other anions preferred stacking via the tranverse of EDOT ring favours better conduction [3].

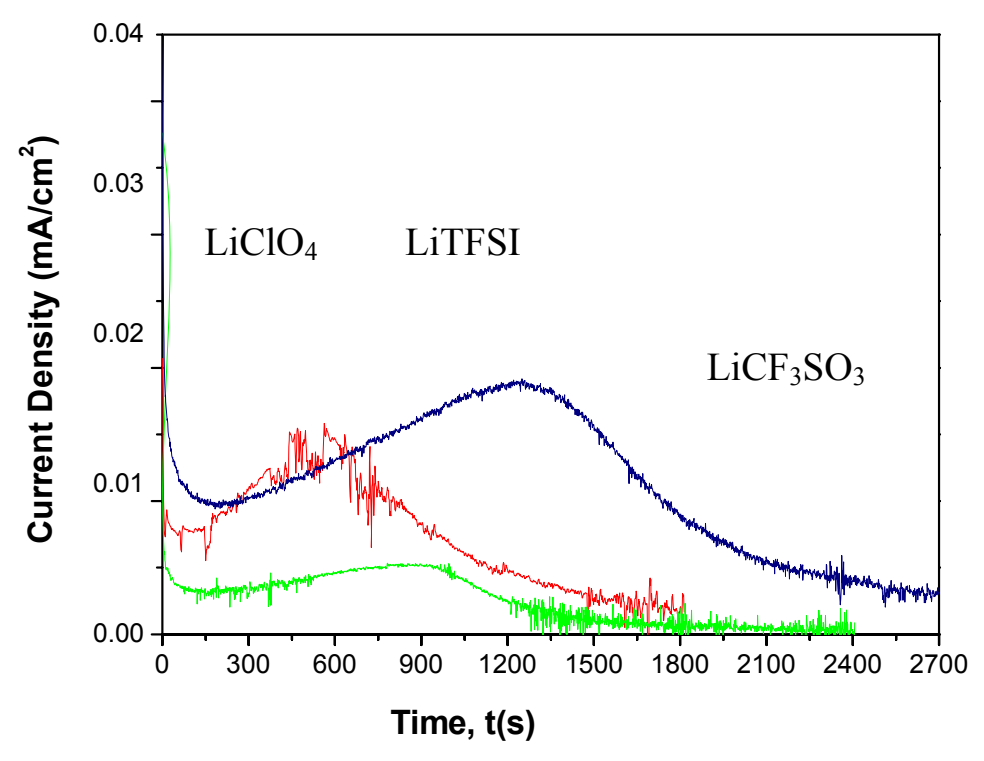

Figure 3.17: Current-time transient for $0.01 \mathrm{M}$ bis-EDOT with different doping anions on dyed $\mathrm{TiO}_{2}$ electrode during in situ photo electrochemical polymerization. 


\subsubsection{Different oligomer}

Replacing bis-EDOT, tris-EDOT was used as an oligomer for photo-electrochemical polymerization of PEDOT. The tris-EDOT has low oxidation potential as compared to bis-EDOT, which can be easily oxidised by ruthenium dye cation. Interestingly, when tris-EDOT oligomer was used for the formation of PEDOT, the charge was calculated as high as $64 \mathrm{mC} / \mathrm{cm}^{2}$. This charge was achieved when the PEP was carried out under $118 \mathrm{~mW} / \mathrm{cm}^{2}$ of light intensity and growth of PEDOT growth time was 30 minutes. However, although more charge was achieved than when using bisEDOT as precursor, the efficiency of the resulting solid-state DSC was limited to less than $1.5 \%$. This may be due to less conductive PEDOT being formed and may be the shorter PEDOT formed. Different light intensities for the photoelectrochemical polymerization were also examined and their total polymerization charge was calculated (Figure 3.18). The colour of the photo-anode became darker, which did not happen in bis-EDOT.

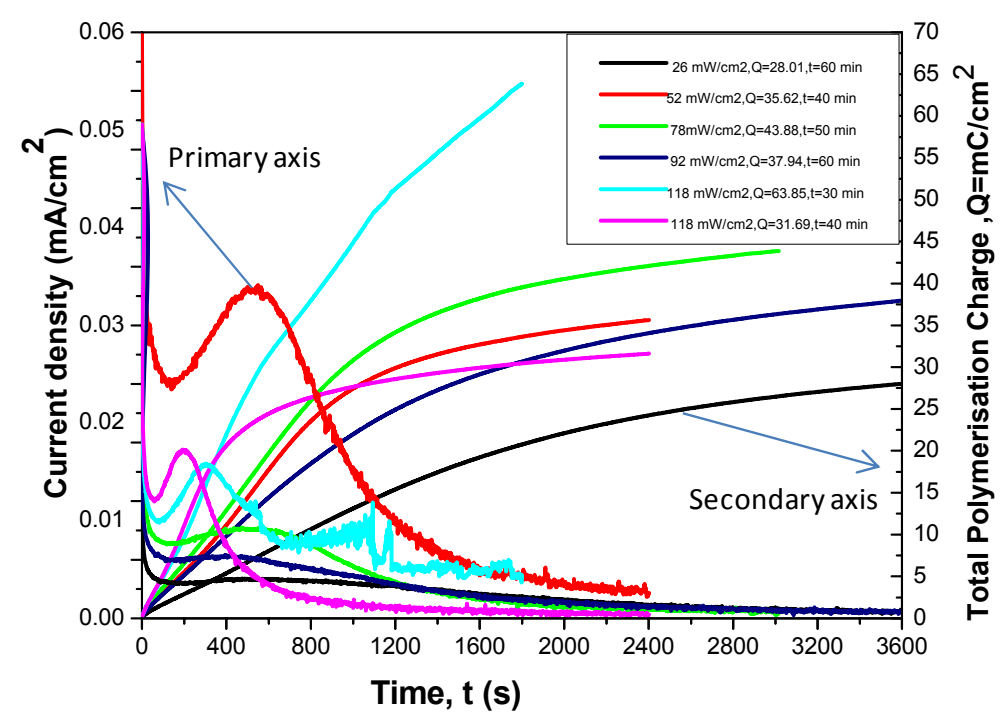

Figure 3.18: Total polymerization charge when different intensities of light $\left(\mathrm{mW} / \mathrm{cm}^{2}\right)$ were used during photo-electrochemical polymerization of PEDOT from tris-EDOT onto dyed $\mathrm{TiO}_{2}$. 


\subsubsection{Solid-state dye-sensitized solar cells testing}

The short circuit current $\left(J_{\mathrm{sc}}\right)$, fill factor $(\mathrm{FF})$ could be improved by increasing the $\mathrm{TiO}_{2}$ pore filling by PEDOT during photo-electrochemical polymerization and by using Goretex ${ }^{\circledR}$-Au-PEDOT as counter-electrode. Goretex ${ }^{\circledR}-A u-P E D O T$ is used as a counter electrode to explore the possibility of fabricating semiflexible solid-state DSC. Goretex ${ }^{\circledR}-\mathrm{Au}-\mathrm{PEDOT}$ has low sheet resistance. This compressible, flexible, highly conductive current-collecting electrode based on vapor-phase polymerized PEDOT on a gold-Goretex ${ }^{\circledR}$ membrane having $60 \%$ compressibility and $80 \%$ porosity make this novel electrode tolerant of defects in the $\mathrm{TiO}_{2}$ photo anode. Its compressibility, flexibility and surface roughness have proven to be an ideal matching current collector counter electrode for the dyed $\mathrm{TiO}_{2}$ (working electrode) surface. Moreover this electrode will be cost effecetive for large scale production for next generation of organic based solid- stae DSCs. Described below are further investigations on the effects of conductivity, various dopants and their concentration on the photo-polymerization.

\subsubsection{Photovoltaic performance measurements (JV and IPCE)}

Sandwich solid-state dye-sensitized solar cells were prepared by clipping the working electrode and counter electrode (Goretex $\left.{ }^{\circledR}-A u-P E D O T\right)$ together mechanically. The current-voltage (JV) curves were measured under 1 sun (100 $\mathrm{mW} / \mathrm{cm}^{2}$ light intensity, 1.5 AM Global), and was calibrated with a standard silicon photo-detector (PECCELL). The incident photon to current conversion efficiency (IPCE) was measured using our in-house set up for IPCE measurements (see Chapter 2. experimental). The IPCE spectra were taken to see the sensitization effect of the dye and PEDOT. 


\subsubsection{Different light intensity during photo-electrochemical polymerization}

The most efficient solid-state DSC was fabricated when the growth time of PEDOT was 30 minutes and $22 \mathrm{~mW} / \mathrm{cm}^{2}$ of light intensity applied during photoelectrochemical polymerization (PEP) in acetonitrile. Efficiencies of all ssDSC increases with the increase of light intensity (from $5 \mathrm{~mW} / \mathrm{cm}^{2}$ to $22 \mathrm{~mW} / \mathrm{cm}^{2}$ ) and decreases when PEP done beyond $22 \mathrm{~mW} / \mathrm{cm}^{2}$ to $200 \mathrm{~mW} / \mathrm{cm}^{2}$ light intensity applied during polymerization. All photovoltaic parameters are presented in Table 3.3. Fill factor of ssDSCs increases with increase of light intensity (from $5 \mathrm{~mW} / \mathrm{cm}^{2}$ to $22 \mathrm{~mW} / \mathrm{cm}^{2}$ ) during photo-electrochemical polymerization; it clearly indicates that the more amount of PEDOT formed which facilitated the better physical and electrical contact between working electrode and counter electrode. This increase of FF trend reversed when PEP done beyond $22 \mathrm{~mW} / \mathrm{cm}^{2}$ light intensity. Transient absorption spectroscopy study suggests that the faster dye regeneration kinetics occurs with increase of PEDOT growth which suggests that short circuit current density $\left(J_{\mathrm{sc}}\right)$ is limited by the slow regeneration kinetics [10]. This slow dye regeneration kinetics competes with recombination reaction between electrons in conduction band of $\mathrm{TiO}_{2}$ and dye cation $\left(\mathrm{Z907}^{+}\right)$which may limit $J_{\text {sc }}[10]$. Higher $J_{\text {sc }}$ values were achieved when the electrical contact between dye, HTM and Goretex ${ }^{\circledR}$ Au-PEDOT counter electrode was good. This facilitated the electron transfer from PEDOT on the counter electrode to the dye molecule in one direction. A blocking layer of $\mathrm{TiO}_{2}$ was incorporated which inhibited short circuits, i.e. back electron transfer and electron leakages from the conduction band of $\mathrm{TiO}_{2}$ and FTO to HTM were reduced. In other words, the shunt resistance of the circuit increased and the series resistance decreased because of unidirectional flow of electron at the heterojunction interface of PEDOT/Dye/TiO 2 . Open-circuit voltage $\left(V_{\mathrm{oc}}\right)$ also decrease with decrease of light intensity and this is due to decrease of the difference between electron density in $\mathrm{TiO}_{2}$ (i.e. Fermi energy level) and electrochemical potential of PEDOT. With increase of PEDOT amount with increase of light intensity, it is evident that the regeneration of dye by PEDOT is not $100 \%$ because the $J_{\text {sc }}$ is not linear with w.r.t. the light intensity of irradiation. The overall photovoltaic performance of ssDSC increases with increases of the light intensity. 
Yanagida et al. have already proposed the controlled distribution of PEDOT into the dyed nanomatrix. This proposition could be very helpful for the fabrication of highly efficient devices [3, 7-9]. Figures 3.19 and 3.20 present the JV curve and IPCE spectra respectively, of the two best solid-state DSCs developed in this Chapter.

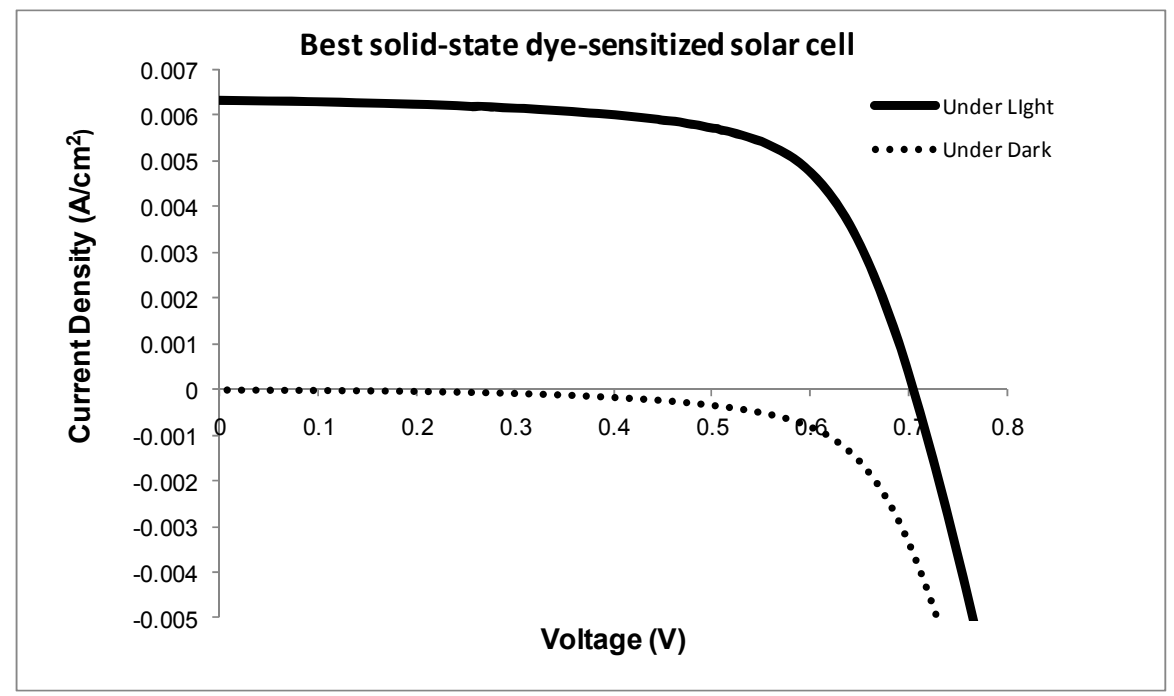

Figure 3.19: Current vs. Voltage plot for one of the best ssDSC with Goretex ${ }^{\circledR}-\mathrm{Au}-$ PEDOT as counter electrode, exhibiting $V_{\text {oc }} 700 \mathrm{mV}, J_{\mathrm{sc}} 6.42$ $\mathrm{mA} / \mathrm{cm}^{2}$, FF 0.67 , and efficiency $(\eta) 3.01 \%$.

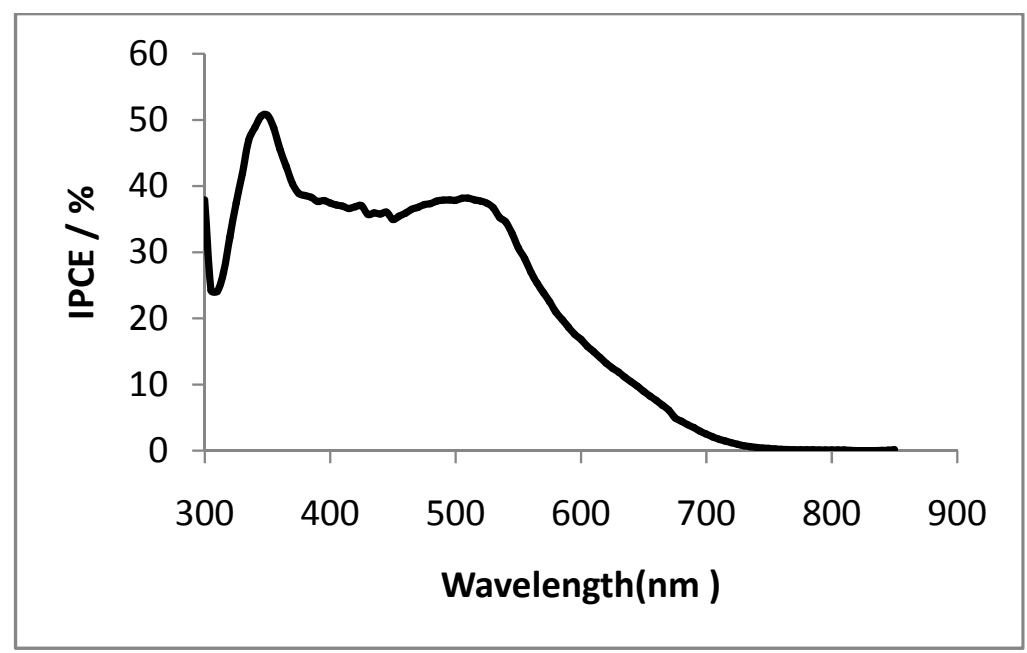

Figure 3.20: IPCE spectrum of one of the another $2.85 \%$ efficient $Z 907$ dyesensitized solid-state solar cells with Goretex ${ }^{\circledR}$-Au-PEDOT as counter electrode. 
Table: 3.3: Photovoltaic performances of ssDSCs when photo anodes prepared under different light intensities during photo-electrochemical polymerization. The light intensity was measured by light meter (Leica 200). The PEDOT deposition time for all photo-anodes was $30 \mathrm{~min}$.

\begin{tabular}{|c|c|c|c|c|}
\hline $\begin{array}{c}\text { Light } \\
\text { intensity } \\
\left(\mathrm{mW} / \mathrm{cm}^{2}\right)\end{array}$ & $V_{\mathrm{oc}}(\mathrm{mV})$ & $\begin{array}{c}J_{\mathrm{sc}} \\
\left(\mathrm{mA} / \mathrm{cm}^{2}\right)\end{array}$ & FF & $(\eta)(\%)$ \\
\hline 5 & 625 & 5.30 & 0.55 & 1.82 \\
\hline 17 & 560 & 6.62 & 0.59 & 2.19 \\
\hline 20 & 610 & 6.73 & 0.58 & 2.40 \\
\hline 21 & 645 & 6.33 & 0.62 & 2.52 \\
\hline 22 & 700 & 6.42 & 0.67 & 3.01 \\
\hline 30 & 700 & 4.95 & 0.60 & 2.24 \\
\hline 75 & 640 & 6.16 & 0.57 & 2.25 \\
\hline 89 & 650 & 5.69 & 0.54 & 1.99 \\
\hline 100 & 610 & 4.52 & 0.48 & 1.33 \\
\hline 200 & 470 & 4.86 & 0.43 & 0.99 \\
\hline
\end{tabular}

\subsubsection{PEDOT growth time}

The variation in performance of solid-state dye-sensitized solar cells using different polymerization time conditions on the dyed $\mathrm{TiO}_{2}$ is presented in Tables 3.4. In table 3.4 , it is observed that when PEDOT is grown for $30 \mathrm{~min}$, we get higher $V_{\mathrm{oc}}, J_{\mathrm{sc}}, \mathrm{FF}$ and overall efficiency of the ssDSCs. This is because of PEDOT is well electronically connected to dyed $\mathrm{TiO}_{2}$. The surface roughness $(\sim 90 \mathrm{~nm})$ of photo anodes were also higher when more PEDOT is grown which faciliated the better physical contact with the higher surface roughness of Goretex ${ }^{\circledR}$-Au-PEDOT $(\sim 300$ $\mathrm{nm}$ ) counter electrode ( 3 order magnitude higher) when both electrodes were clipped together mechanically (Details given in Chapter 4). 
Table 3.4: Summary of solid-state dye sensitized solar cells with Z907 dye as sensitizer with Goretex ${ }^{\circledR}-A u-P E D O T$ was used as counter electrode. Liquid DSC also compared with ssDSCs.The intensity of light during the growth of PEDOT films was $22 \mathrm{~mW} / \mathrm{cm}^{2}$.

\begin{tabular}{|c|c|c|c|c|}
\hline $\begin{array}{c}\text { PEDOT } \\
\text { growth (min) }\end{array}$ & $V_{\mathrm{oc}}(\mathrm{mV})$ & $J_{\mathrm{sc}}\left(\mathrm{mA} / \mathrm{cm}^{2}\right)$ & FF & $(\eta)(\%)$ \\
\hline 30 & 680 & 5.96 & 0.71 & 2.86 \\
\hline 15 & 620 & 1.62 & 0.64 & 0.64 \\
\hline 8 & 444 & 0.84 & 0.42 & 0.16 \\
\hline 4 & 465 & 0.27 & 0.82 & 0.10 \\
\hline Liquid DSC & 755 & 8.42 & 0.63 & 4.00 \\
\hline
\end{tabular}

Dye-sensitized solar cells were also fabricated for comparison, using a mixture of 0.10 M LiI, 0.05 $\mathrm{M} \mathrm{I}_{2}, 0.60 \mathrm{M}$ DMPII, $0.50 \mathrm{M}$ t-BP in mixed 85:15 actetonitrile: valeronitrile solvent (as the electrolyte). Their average PV performance is presented in Table 3.4. It is seen that their efficiency is somewhat higher than the optimized ssDSCs. Liquid DSCs which were fabricated during this chapter, we did not use any scattering $\mathrm{TiO}_{2}$ layer on top of the mesoporous $\mathrm{TiO}_{2}$ layer. This is done because to see the comparison in performance between solid DSCs and liquid DSCs with the same thickness of $\mathrm{TiO}_{2}(5.5 \mu \mathrm{m})$. Moreover, the thickness of $\mathrm{TiO}_{2}$ (in our case 5.5 $\mu \mathrm{m}$ ) which is $\sim 3$ times less than what used in standard DSCs.

For standard Liquid DSCs cells, to enhance the scattering effect, larger particles are incorporated on top of the mesoporous $\mathrm{TiO}_{2}$ layer to increase the optical path inside the nano-porous $\mathrm{TiO}_{2}$ matrix which is known as scattering $\mathrm{TiO}_{2}$ layer. It has been reported by Nazeeruddin et.al [ChemSuSChem 2008, 1, 699-707] that larger particles with lower internal surface area harvest more photons compared to smaller particles. Scattering particles always helps in Light harvesting efficiency (LHE) which leads more photo-current in the device. 


\subsubsection{Dependence of ssDSC performance on $\mathrm{TiO}_{2}$ layer thickness}

The thickness of the $\mathrm{TiO}_{2}$ layer was controlled by putting a number of layers of Scotch tape as masks on the top of the FTO glass substrate. The thickness of each $\mathrm{TiO}_{2}$ layer was measured by a surface profilometer (Dektak Profilometer Veeco-3). Table 3.5 summarises the effect of varying the thickness of $\mathrm{TiO}_{2}$ on the PV performance of the solid-state DSCs. It is seen that $5.5 \mu \mathrm{m}$ thickness of $\mathrm{TiO}_{2}$ increased the PV parameters of $J_{\mathrm{sc}}, V_{\mathrm{oc}}$ and overall efficiency. It is observed that this $5.5 \mu \mathrm{m}$ thickness is the optimal thickness for better performance of solid-state dyesensitized solar cells.

The overall performances of solid-state DSC with various thicknesses of $\mathrm{TiO}_{2}$ and Goretex ${ }^{\circledR}-A u-P E D O T$ as counter electrode are presented in Table 3.5.

Table 3.5: Effect of variation of thickness of mesoporous $\mathrm{TiO}_{2}$ layer on the $\mathrm{PV}$ performance of a solid-state DSC with Z907 dye used as photo-sensitizer and Goretex ${ }^{\circledR}-\mathrm{Au}-\mathrm{PEDOT}$ as counter electrode (under 1.5 Air Mass (AM1.5) condition).

\begin{tabular}{|c|c|c|c|c|}
\hline $\begin{array}{c}\mathrm{TiO}_{2} \text { Thickness } \\
(\mu \mathrm{m})\end{array}$ & $V_{\mathrm{oc}}(\mathrm{mV})$ & $J_{\mathrm{sc}}\left(\mathrm{mA} / \mathrm{cm}^{2}\right)$ & $\mathrm{FF}$ & $(\eta)(\%)$ \\
\hline 7 & 645 & 5.95 & 0.58 & 2.21 \\
\hline 7 & 670 & 5.25 & 0.63 & 2.21 \\
\hline 7 & 630 & 4.85 & 0.63 & 1.92 \\
\hline 5.5 & 675 & 6.03 & 0.69 & 2.80 \\
\hline 5.5 & 665 & 6.02 & 0.66 & 2.63 \\
\hline 5.5 & 685 & 7.14 & 0.54 & 2.63 \\
\hline 3 & 595 & 4.41 & 0.49 & 1.21 \\
\hline 3 & 635 & 4.44 & 0.53 & 1.50 \\
\hline 3 & 605 & 3.84 & 0.56 & 1.30 \\
\hline
\end{tabular}




\subsubsection{PEDOT in ionic liquid}

When bis-EDOT was dissolved in the ionic liquid (EMITFSI), photo-polymerization proceeded for a longer time and the photo-anode became very dark, i.e. more PEDOT was grown. However, the solid-state DSCs obtained were 90\% less efficient with $V_{\mathrm{oc}}=565 \mathrm{mV}, J_{\mathrm{sc}}=0.32 \mathrm{~mA} / \mathrm{cm}^{2}, \mathrm{FF}=0.57$, efficiency $=0.10 \%$ (see Table 3.6). The efficiency of the device was limited to less than $0.5 \%$ when the photoanode was prepared in ionic liquid medium. We also tried replacing the acetonitrile and bis-EDOT solution. This solution was prepared directly with ionic liquid which contains LiTFSI, EMITFSI, and tert-butyl pyridine. The PV parameters achieved with the solid-state DSCs using different anions are summarised in Table 3.6. The low efficiency of device may be due to the low conductivity of PEDOT or the low ratio of dye to PEDOT. More PEDOT was formed on surface rather than nanostructured dyed $\mathrm{TiO}_{2}$ pore with this ionic liquid. This can be confirmed through the nature of the photo-current during the growth of PEDOT. If the PEDOT grown into the nanomatrix the photo-current increases then after sometimes it decline to zero where in case of ionic liquid the nature of the curve looks without any trend of increase or decrease seems to be the growth like PEDOT grown on FTO glass substrate (potentiostaticaly grown). For better efficiency, photo-anode needs more transparent and conductive PEDOT in the pores rather than on the surface of dyed $\mathrm{TiO}_{2}$.

Transparency is another issue for the photo-anode, since the deposited films of the photo-anode became darker because of thicker PEDOT. This dark black colour PEDOT restricted harvesting more photons when irradiated by solar simulator. The decreases of photons lower the $J_{\text {sc }}$ because the number of excited dye decreases with decrease of photons. Similarly, Voc decreases with decrease of photon because of decrease of electron density in $\mathrm{TiO}_{2}$ which lowers the Fermi energy level of $\mathrm{TiO}_{2}$. Therefore the overall energy difference between Fermi level of $\mathrm{TiO}_{2}$ and the HOMO of PEDOT decreases. 


\subsubsection{Influence of different dopant anions used during photo-electrochemical polymerization}

Various dopant anions (see Figure 3.21) were also introduced into the bis-EDOT solution for photo-electrochemical polymerization of PEDOT on the dyed $\mathrm{TiO}_{2}$ working electrode. The effect of replacing the acetonitrile solvent for the bis-EDOT precursor with the ionic liquid EMITFSI containing LiTFSI and tert-butyl pyridine was also investigated. $\mathrm{LiClO}_{4}$ was the best dopant anion; which worked better for PV performances. Among the dopant anions studied, $\mathrm{ClO}_{4}{ }^{-}$was seen to be the best in terms of device performance. It was further (Table 3.6) observed that when bisEDOT was mixed with $\mathrm{LiClO}_{4}$ and LiTFSI individually efficiencies of $3 \%$ and $2.65 \%$, were achieved respectively.

Table 3.6: Summary of the PV performance of solid-state DSCs with various dopant anions incorporated on the PEDOT in the photo-anode during the photoelectrochemical polymerization step; and the influence of using an ionic liquid in the polymerization. All photo-anodes were irradiated with a light intensity of 22 $\mathrm{mW} / \mathrm{cm}^{2}$.

\begin{tabular}{|c|c|c|c|c|c|}
\hline $\begin{array}{c}\text { Different } \\
\text { dopant anions }\end{array}$ & $\begin{array}{c}V_{\mathrm{oc}} \\
(\mathrm{mV})\end{array}$ & $\begin{array}{c}J_{\mathrm{sc}} \\
\left(\mathrm{mA} / \mathrm{cm}^{2}\right)\end{array}$ & $\begin{array}{c}\text { Fill } \\
\text { Factor } \\
(\mathrm{FF})\end{array}$ & $\begin{array}{l}\text { Efficiency } \\
\text { (१) } \%\end{array}$ & $\begin{array}{l}\text { Counter } \\
\text { electrode }\end{array}$ \\
\hline $\mathrm{LiClO}_{4}$ & 700 & 6.42 & 0.67 & 3.01 & $\begin{array}{l}\text { PEDOT-Au- } \\
\text { Goretex }\end{array}$ \\
\hline LiTFSI & 710 & 6.01 & 0.62 & 2.64 & $\begin{array}{l}\text { PEDOT-Au- } \\
\text { Goretex }\end{array}$ \\
\hline $\mathrm{LiCF}_{3} \mathrm{SO}_{3}$ & 685 & 5.26 & 0.50 & 1.82 & $\begin{array}{l}\text { PEDOT-Au- } \\
\text { Goretex }\end{array}$ \\
\hline $\begin{array}{l}\text { tert-butyl ammonium } \\
\text { hexafluorophosphate }\end{array}$ & 590 & 2.34 & 0.50 & 0.69 & $\begin{array}{l}\text { PEDOT-Au- } \\
\text { Goretex }\end{array}$ \\
\hline EMI.TFSI (Ionic liquid) & 645 & 0.19 & 0.94 & 0.12 & $\begin{array}{l}\text { PEDOT-Au- } \\
\text { Goretex }\end{array}$ \\
\hline $\begin{array}{c}\text { tetrabutylammonium } \\
\text { tetrafluoroborate }\end{array}$ & 130 & 2.09 & 0.24 & 0.06 & $\begin{array}{c}\text { PEDOT-Au- } \\
\text { Goretex }\end{array}$ \\
\hline
\end{tabular}




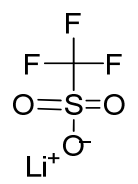

Lithium trifluoromethanesulphonate

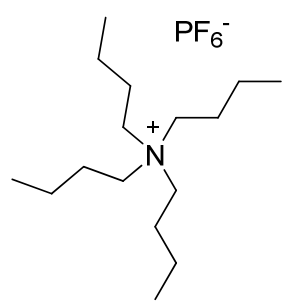

Tetrabutyl ammonium hexafluoride phosphate

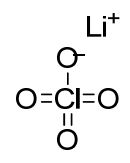

Lithium perchlorate

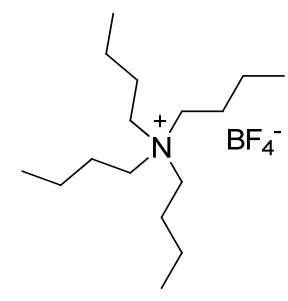

Tetrabutyl ammonium hexafluoride borate

LiTFSI (Lithium bis[(perfluoromethyl)sulfonyl]imide

Figure 3.21: The different dopant anions used during photo-electrochemical polymerization of bis-EDOT in preparation of photo-anode.

The purpose of introducing different dopant anions was to enhance the conductivity (electronic and ionic) of PEDOT. These different anions are seen from Table 3.6 to have great influence on both the JV curves and conductivity. Electrochemical impedance analysis studies have been carried out by Yanagida et al. [8] with different anions to understand the equivalent circuit (e.g. shunt resistance, series resistance, conductivity, etc.) of solid-state dye-sensitized solar cells. It had been reported by Yanagida [8] that LiTFSI was the best dopant anion for the successful electrochemical synthesis of PEDOT, enhancing the conductivity of PEDOT. 


\subsubsection{Different oligomer}

The preliminary results of photovoltaic performances such as JV curve as well as IPCE for an analogous solid-state DSC fabricated using tris-EDOT instead of bisEDOT for the photo-electrochemical polymerization step are presented in Figure 3.22 and Figure 3.23. The PV parameters obtained when this PEP was done under different light intensities are shown in Table 3.7. It was observed that when the PEP was done under $118 \mathrm{~mW} / \mathrm{cm}^{2}$ illumination for 30 minutes it gave better device performance.

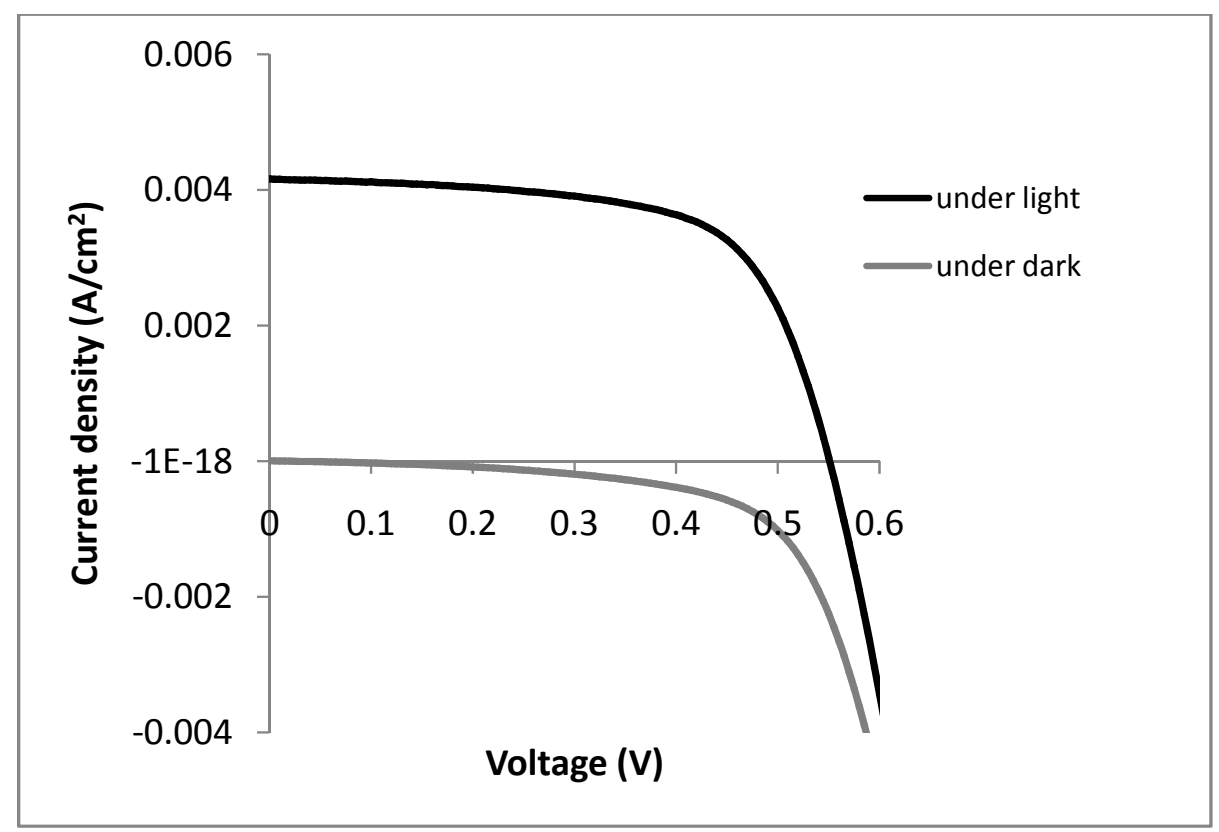

Figure 3.22: $J V$ curve for a Z907-dye-sensitized solid-state solar cell with Goretex ${ }^{\circledR}-A u-P E D O T$ counter electrode, having PV parameters as $V_{\mathrm{oc}}$ $=555 \mathrm{mV}, J_{\mathrm{sc}}=4.16 \mathrm{~mA} / \mathrm{cm}^{2}, \mathrm{FF}=0.64$ and efficiency $(\eta)=1.49 \%$. Here tris-EDOT was used as starting material for PEDOT formation instead of bis-EDOT. 


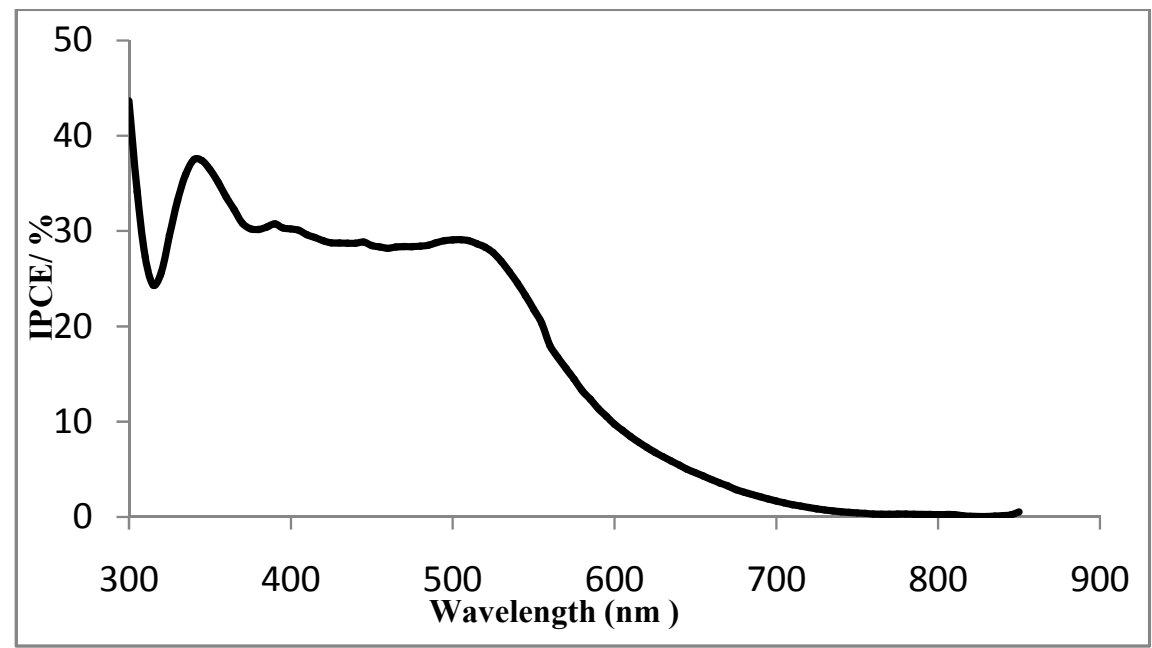

Figure 3.23: IPCE curve for one of the best (1.49\% efficient) Z907 dye-sensitized solid-state solar cells with Goretex ${ }^{\circledR}$-Au-PEDOT as counter electrode. Here tris-EDOT was used as the starting oligomer instead of bisEDOT during PEP.

Table 3.7: PV performances of solid-state DSCs prepared using different light intensities during PEP (Here tris-EDOT was used as oligomer for polymerization instead of bis-EDOT). Goretex ${ }^{\circledR}$-Au-PEDOT counter electrode in each case.

\begin{tabular}{|c|c|c|c|c|c|c|}
\hline $\begin{array}{c}\text { Intensity of } \\
\text { light } \\
\left(\mathrm{mW} / \mathrm{cm}^{2}\right)\end{array}$ & $\begin{array}{c}\text { Time of } \\
\text { PEDOT } \\
\text { growth }\end{array}$ & $\begin{array}{c}\text { Total charge } \\
\text { calculated } \\
\left(\mathrm{mC} / \mathrm{cm}^{2}\right)\end{array}$ & $\begin{array}{c}V_{\mathrm{oc}} \\
(\mathrm{mV})\end{array}$ & $\begin{array}{c}J_{\mathrm{sc}} \\
\left(\mathrm{mA} / \mathrm{cm}^{2}\right)\end{array}$ & \begin{tabular}{c} 
FF \\
\hline 26
\end{tabular} & $\begin{array}{c}\text { Efficiency } \\
(\%)\end{array}$ \\
\hline 52 & $40 \mathrm{~min}$ & 28.0 & 370 & 3.83 & 0.59 & 0.84 \\
\hline 78 & $50 \mathrm{~min}$ & 43.6 & 475 & 5.28 & 0.59 & 1.47 \\
\hline 92 & $60 \mathrm{~min}$ & 37.9 & 385 & 4.53 & 0.60 & 1.04 \\
\hline 118 & $30 \mathrm{~min}$ & 63.8 & 555 & 4.16 & 0.64 & 1.49 \\
\hline 118 & $40 \mathrm{~min}$ & 31.7 & 520 & 4.04 & 0.47 & 1.00 \\
\hline
\end{tabular}




\subsubsection{Dependence on light intensities of ssDSC performance}

The light intensity dependence of PV parameters of solid-state DSCs was investigated to calculate the loss mechanism and counter electrode performance. The current density-voltage curves were measured using various illumination intensities (using different neutral density filters) for ssDSCs with a $\sim 5.5 \mu \mathrm{m} \mathrm{TiO}_{2}$ layer prepared in optimal conditions of PEP and are shown in Figure 3.24. The measured photovoltaic parameters were plotted against the different light intensities employed. Linear light intensity dependency of the short circuit current of the cell was not observed. Current density $\left(J_{\mathrm{sc}}\right)$ decreases with decrease of light intensity of irradiation of cells and this occurs because the number of excited dye decreases with the decrease of light intensity. Open circuit voltage $\left(V_{\text {oc }}\right)$ also a decrease with decrease of light intensity and this is because of decrease of electron density in $\mathrm{TiO}_{2}$ (i.e. Fermi energy level decreases). From Figure 3.24, it is evident that the regeneration of dye by PEDOT is not $100 \%$ because the $J_{\mathrm{sc}}$ is not linear with w.r.t. the light intensity of irradiation. The overall photovoltaic performance of ssDSC increases with increases of the light intensity.

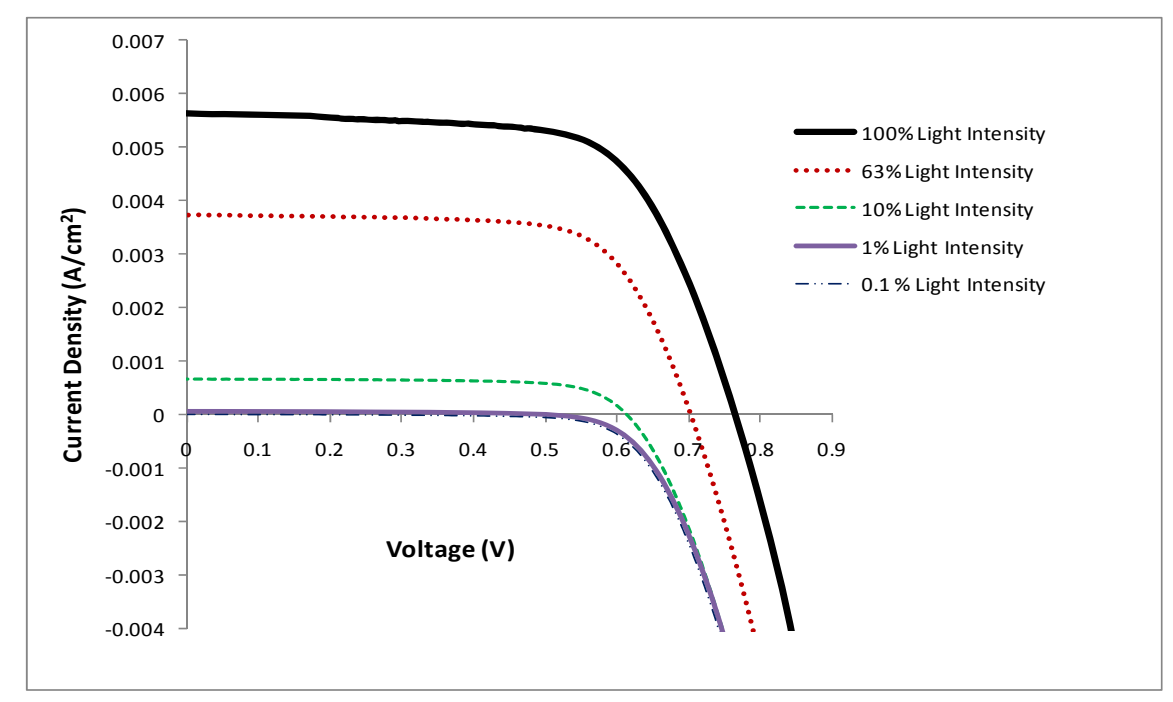

Figure 3.24: Current density vs. voltage curves obtained using various light intensities illumination for solid-state DSCs. Au/ITO used as counter electrode. 


\subsubsection{Stability of the ssDSC}

The stability and reproducibility of ssDSCs (using Z907 dye and a Goretex ${ }^{\circledR}$-AuPEDOT as counter electrode) were measured periodically over one month (Table 3.8). The photo-anode and counter electrode were both kept separately in a dark drawer. For the measurements at each of the time intervals, the working electrode and counter electrode were clipped together when measuring their PV performance. It is observed that the overall conversion efficiency of the device decreased with increase of days. This happened because some of the PEDOT came out from photoanode because of mechanical handling of the device. This is because of several times of clipping of both photo-anode and counter electrode together whenever we measured the device each time. The performances decreased because of extra handling and may be exposure to air.

Table 3.8: Summary of solid state devices with Z907 dye sensitizer with Goretex ${ }^{\circledR}$ $\mathrm{Au}-\mathrm{PEDOT}$ as counter electrode.

\begin{tabular}{|c|c|c|c|c|}
\hline No of Days & $\begin{array}{c}V_{\mathrm{oc}} \\
(\mathrm{mV})\end{array}$ & $\begin{array}{c}J_{\mathrm{sc}} \\
\left(\mathrm{mA} / \mathrm{cm}^{2}\right)\end{array}$ & $\begin{array}{c}\text { FF } \\
(\%)\end{array}$ \\
\hline 0 & 700 & 6.42 & 0.67 & 3.01 \\
\hline 6 & 695 & 5.79 & 0.66 & 2.65 \\
\hline 7 & 690 & 5.62 & 0.62 & 1.49 \\
\hline 22 & 630 & 2.30 & 0.68 & 0.99 \\
\hline 29 & 640 & 3.48 & 0.67 & 0.62 \\
\hline
\end{tabular}

\section{4 Conclusions}

PEDOT based solid-state dye-sensitized solar cells have been successfully fabricated using in situ photo-electrochemically polymerized PEDOT as a hole transporting material. The power conversion efficiency of the best device was $3.01 \%$. This is higher than the best result reported to date in the literature. The Goretex ${ }^{\circledR}-\mathrm{Au}$ - 
PEDOT counter electrode developed in this chapter gave an even better efficiency. A high value of $J_{\mathrm{sc}}\left(6.42 \mathrm{~mA} / \mathrm{cm}^{2}\right)$ is also achieved in the best device which may be attributed to the good charge transport property and high transparency of PEDOT. It is found that 30 minutes of PEDOT growth leads to the best device performance when using $22 \mathrm{~mW} / \mathrm{cm}^{2}$ light intensity during the photo-electrochemical polymerization of PEDOT.

The polymerization conditions have been optimised with the thickness of $\mathrm{TiO}_{2}$, period of PEDOT growth (i.e. amount of PEDOT), nature of dopant anion, and solvent. The stability as well as the reproducibility of the devices has also been established. Comparison of the performance of these novel ssDSCs with dyesensitised solar cells with the same thickness of $\mathrm{TiO}_{2}$ and using $\mathrm{I}^{-} / \mathrm{I}_{3}^{-}$electrolyte, indicates that the solid-state DSCs have a $25 \%$ lower value of $J_{\mathrm{sc}}$ and efficiency than liquid DSCs.

The use of tris-EDOT instead of bis-EDOT as the oligomer for the photoelectrochemical deposition of PEDOT for the photo-anode is seen to limit the efficiency of the resulting ssDSC to $1.49 \%$. This may be attributed to the PEDOT formed from tris-EDOT not being conductive as that from bis-EDOT. The conductivity of conducting polymer increases when low oligomer used as a starting material as compared to its higher analogues. Transparency is another issue for the photo-anode, since the deposited films of the photo-anode become darker in the case of tris-EDOT.

Detailed investigations and further optimisation conditions will lead the way for further improvement of PV performances for the tris-EDOT system. A crucial goal will be to further enhance the PV performance, which needs proper facilitation of charge separation and transport at each interface. For this, further possibilities of using different dyes and the polymerization conditions will be discussed in Chapter 6. 


\section{5 References}

1. Chiba, Y., Islam A., Watanabe, Y., Komiya, R., Koide N., and Han, L., Japanese Journal of Applied Physics, 45 (2006) 638-640.

2. Yanagida, S., Yu, Y., and Manseki, K., Accounts of Chemical Research, 42 (2009) 1827-1838.

3. Xia, J., Masaki, N., Lira-Cantu, M., Kim, Y., Jiang, K., and Yanagida S., Journal of the American Chemical Society, 130 (2008) 1258-1263.

4. Fukuri, N., Saito, Y., Kubo, W., Senadeera G.K.R, Kitamura, T., Wada, Y., and Yanagida, S., Journal of the Electrochemical Society, 151 (2004) 1745-1748.

5. Fukuri, N., Masaki, N., Kitamura, T., Wada,Y., and Yanagida, S., Journal of Physical Chemistry B, 110 ( 2006) 25251-25258.

6. Saito, Y., Fukuri, N., Senadeera, R., Kitamura, T., Wada, Y., and Yanagida S., Electrochemical Communications, 6 (2004) 71-74.

7. Murakoshi, K., Kogure, R., Wada, Y., and Yanagida, S., Chemistry Letters, 26 (1997) 471.

7a. Hallett A. J., and Jones J.E., Dalton Transactions, 40 (2011) 3871.

8. Yu, Q., Liu, S., Zhang, M., Cai, N., Wang, Y., and Wang, P., Journal of Physical Chemistry C, 113 (2009)14559-14566.

9. Turbiez, M., Frere, P., Allain, M., Gallego-Planas, N., and Roncali, J., Macromolecules, 38 (2005) 6806-6812

10. Mozer, A. J., Panda, D. K., Gambhir, S., Winther-Jensen, B., and Wallace, G.G., Journal of the American Chemical Society, 132 (2010) 9543-9545. 
11. Ma, L., Li, Y., Yu, X., Yang, Q., and Noh, C, Solar Energy Materials and Solar Cells, 92 (2008) 1253-1259.

12. Ahmad, S., Deepa, M., and Singh, S, Langmuir, 23 (2007) 11430-11433. 


\section{MORPHOLOGICAL DETERMINATION OF PHOTO-ANODE GROWN BY A PHOTO- ELECTROCHEMICAL METHOD}




\section{1 Introduction}

In this chapter, fundamental issues will be discussed about the low efficiency of solid-state dye-sensitized solar cells (ssDSCs). The influence of the morphology of the working electrode (photo-anode) on photovoltaic performance of the device with spiro-OMeTAD based ssDSCs [1-2, 3-7] has been reported. However, no morphological studies of poly (ethylenedioxythiophene) (PEDOT) based ssDSCs have been described to date. This chapter will discuss about the PEDOT growth into photo-anode. The photovoltaic performance of the device has been examined with regard to the amount of PEDOT and the morphology of PEDOT in photo-anodes.

The deposition of PEDOT into dyed $\mathrm{TiO}_{2}$ substrates was carried out by an in situ photo-electrochemical polymerization technique described in Chapter 3. The growth of PEDOT was performed under various conditions: different light intensities, different dopants, different solvents, different thickness of $\mathrm{TiO}_{2}$, different electrolytes (including the ionic liquid EMITFSI / LiTFSI), and different periods of PEDOT growth (see Chapter 3). In the present chapter the photo-anode was prepared under the conditions optimised in Chapter 3, namely $22 \mathrm{~mW} / \mathrm{cm}^{2}$ light intensity, 5.5 $\mu \mathrm{m} \mathrm{TiO}_{2}$ thickness, $\mathrm{LiClO}_{4}$ dopant, acetonitrile as electrolyte, and 30 minutes PEDOT growth. These conditions consistently led to 3\% efficient devices. Further, photo-anodes were also prepared using different periods of growth of the PEDOT, while maintaining the other conditions constant. The morphology of each of these photo-anodes was studied by scanning electron microscopy (SEM), SEM-Energy Dispersive X-ray Analyser and atomic force microscopy (AFM), and was correlated with their photovoltaic performance.

These fundamental issues were further elucidated by studying the growth of PEDOT into dyed $\mathrm{TiO}_{2}$ substrates, determining the morphology of each photo-anode (by SEM, AFM), optical absorbance and conductivity (by UV-Vis-NIR and Raman), establishing the number of bis-EDOT repeating units and the molecular weight distribution in the photo-anodes produced where the PEDOT polymer has been grown for varying periods (by Matrix assisted laser desorption ionisation (MALDI) 
Mass spectrometry). Recently, Wood et al. [8] reported a solventless MALDI technique used for insoluble or slightly soluble species that involves the use of only solid analyte and matrix during sample preparation. They found that the use of this technique for solid polyaniline resulted in mass spectra that were similar to the spectra of the soluble oligomers. Polyaniline fragments with dissimilar end groups were detected depending on the matrix used. The matrix also affects the percentages of benzenoid and quinoid units in the oligomers. Thus, solventless MALDI appears to be a promising new technique for the mass spectrometric analysis of PEDOT.

The dye regeneration kinetics is also studied in this chapter using transient absorption spectroscopy. All these studies were designed to provide an understanding of the lower photovoltaic performance of the ssDSCs compared to Grätzel cells or spiro-OMeTAD based ssDSCs.

\section{2 Methods and experiments}

The photo-anode films were prepared on fluorine doped tin oxide (FTO) glass substrate using the in situ photo-electrochemical polymerization method. The compact/blocking $\mathrm{TiO}_{2}$ layer and then subsequently the mesoporous $\mathrm{TiO}_{2}$ layer were deposited on FTO using the standard procedure described in sections 2.3.2 and 2.3.3 of Chapter 2. After sintering, the substrates were immersed in the Z907 dye solution for $24 \mathrm{hrs}$, as described in section 2.3.4 (Chapter 2). Photo-electrochemical polymerization was carried out using the method described in 3.2.2.5 (Chapter 3).

The photo-anode films were characterised by UV-Vis-NIR, Raman, MALDI (ToF) mass spectra, scanning electron microscopy (SEM), SEM-EDXA (Energy Dispersive X-ray Analysis), and atomic force microscopy (AFM). The details of these characterisation tools have been described in section 2.5 (Chapter 2). Raman mapping was done with $\mathrm{x}-\mathrm{y}$ resolution of $1 \mu \mathrm{m}$ over a particular area of the PEDOT film. 
The molecular weight distribution of PEDOT (poly bis-EDOT) in photo-anodes produced in this chapter were analysed by this new technique of solventless MALDI using a Shimadzu Axima Confidence instrument. Photo-anode (PEDOT/dye/ $\mathrm{TiO}_{2}$ ) films of approximately $1 \mathrm{~cm}^{2}$ were scraped from their glass backing with a razor blade into a small agate mortar. Approximately 50 times the mass of 2, 5dihydroxybenzoic acid (DHB) was added and the contents mixed with a spatula then ground with an agate pestle to a fine powder. A small quantity of the powder was picked up on the tip of a fine spatula and smeared on to the MALDI target. Compressed air was then used to blow away any loose material. Spectrometer power settings varied between 72 and 96 arbitrary units and $m / z$ optimization settings were set to approximately $50 \%$ of the highest detectable mass for each sample. Spectra were collected in 1000 profile lots with 10 laser shots per profile, and then smoothed using a 10 channel filter width and the baseline subtracted with a filter width of 500 channels. The dye regeneration kinetics was studied by using transient absorption spectroscopy technique with the help of Dr. Attila J Mozer (IPRI, university of Wollongong).

Before assembly of the devices, photo-anode films were rinsed with acetonitrile and subsequently treated for $24 \mathrm{hrs}$ with 1-ethyl-3-methylimidazolium bistrifluoromethanesulfonamide containing $0.2 M$ lithium bistrifluoromethanesulfonamide and 0.2M 4-tert-butylpyridine (t-BP). The sandwichtype devices were completed by clipping the counter electrode (Goretex ${ }^{\circledR}-\mathrm{Au}$ PEDOT) to the photo-anode. Goretex ${ }^{\circledR}$-Au-PEDOT electrode was used as counter electrode in this chapter.

\section{3 Results and discussion}

\subsubsection{PEDOT optical absorption studies by UV-Vis-NIR}

UV-Vis-NIR spectroscopy is a well-established technique for studying optical absorbance and reflectance. This technique has been employed to characterise the photo-anode to obtain information regarding the dye/ PEDOT absorption, loss mechanism and optical transparency and the amount of PEDOT present in the photo- 
anode. The doping level of PEDOT has been examined when the PEDOT is grown with different periods of growth in photo-anode. Figures 4.1, 4.2 and 4.3 present the UV-Vis, NIR and UV-Vis-NIR absorption spectra of various photo-anodes containing $\mathrm{FTO} / \mathrm{TiO}_{2} / \mathrm{Z} 907$ dye/ PEDOT. The amount of PEDOT deposited was varied by using different lengths of time for the photo-electrochemical polymerization of bis-EDOT. In the absence of PEDOT, the absorption up to near $750 \mathrm{~nm}$ (Figure 4.3) is attributed to the absorption of the dye on $\mathrm{TiO}_{2}$, while the absorption at $>1600 \mathrm{~nm}$ is due to the absorption/ reflection of FTO glass substrate and $\mathrm{TiO}_{2}$ (Figure 4.3). In our JACS paper [9], we have reported that when the transient absorption spectrum of $\mathrm{TiO}_{2} / \mathrm{Z907}$ films at various time delays after the laser pump, it has observed that the spectrum between $600 \mathrm{~nm}$ to $900 \mathrm{~nm}$ is attributed to ${\mathrm{Z} 907^{+}}$absorption, with a gradually increasing small and long-lived absorption extending to the IR and attributed to absorption by electrons in the $\mathrm{TiO}_{2}$ films. When PEDOT was deposited into dyed $\mathrm{TiO}_{2}$, a characteristic absorption band was observed at $>650 \mathrm{~nm}$ which is due to doped (oxidised) PEDOT. The difference in the absorption spectrum $(\lambda=300-900 \mathrm{~nm})$ of dyed $\mathrm{TiO}_{2} / \mathrm{PEDOT}$ compared with dyed $\mathrm{TiO}_{2}$ is only $1-2 \%$ [9], indicating a thin $(80-100 \mathrm{~nm})$ PEDOT film is present in photo-anode.

The difference in the absorption spectrum of dyed $\mathrm{TiO}_{2} /$ PEDOT compared with dyed $\mathrm{TiO}_{2}$ was used to quantify a thin $(80-100 \mathrm{~nm})$ PEDOT film was presented in the photo-anode. The estimation was done through UV-Vis-NIR spectra. It has been earlier reported by Reynolds et.al [9a] that doped PEDOT (like as Fig 4.3) is around $10^{5} \mathrm{~cm}^{-1}$. The absorbance of our photo-electrochemically deposited PEDOT (photoanode) is around 0.8 and shown in Figure 4.3 which corresponds to $80 \mathrm{~nm}$ thickness of PEDOT. As the PV performances of our solid-state DSCs give reasonably 3\% efficiency, it clearly demonstrates that some of the PEDOT grown into the nanomatrix of dyed $\mathrm{TiO}_{2}$ photo-anode rather than whole PEDOT grown on to the surface. If this is not the case than, the photovoltaic performance would be limited to the surface layer/counter electrode [9b].

The overall thickness of PEDOT film in the photo-anode is taking into accountability of both the PEDOT grown inside the pores as well as on the surface of dyed $\mathrm{TiO}_{2}$ 
film. The photo-anode discussed here is 30 min PEDOT grown. It has been observed from SEM surface images of photo-anode that in addition to the PEDOT formed within the pores of dyed $\mathrm{TiO}_{2}$ nanomatrix, a discontinuous surface PEDOT layer is also formed. Therefore the thickness of PEDOT is not only in the mesopores of the $\mathrm{TiO}_{2}$, so there is no question of uniformity, hence the full infiltration of PEDOT needs to be matter of concern here.

The electronic spectra (Figure 4.1) exhibited by the dye complex (Z907) is mainly due to metal-to-charge transfer (MLCT) in the visible range whereas ligand centred (LC) $\pi-\pi^{*}$ and $n-\pi^{*}$ transition happens in near UV range. The LC absorbance is more intense than the MLCT absorbance. Figure 4.3 shows that when PEDOT was deposited into dyed $\mathrm{TiO}_{2}$, the characteristic absorption peak at $518 \mathrm{~nm}$ of $\mathrm{Z907}$ dye blue shifted. This may be due to the solvatochromism effect of the Z907 dye [9-10]. The deposition of PEDOT into dyed $\mathrm{TiO}_{2}$ was done in a different electrolyte to that used for the dye uptake measurement. In the case of spiro-OMeTAD based ssDSCs, this kind of blue shift was also observed [11]. In Figure 4.3, the absorption peak of the Z907 dye decreased slightly with an increase in the amount of PEDOT. It was found that when more PEDOT was grown into the photo-anode, the total polymerization charge increased. It was also observed that when the PEDOT was grown for 30 minutes, the photovoltaic performance improved. It is also observed that absorption peak for PEDOT at 2 minutes is almost similar to the absorption peak of the photo-anode having no PEDOT.

From UV-Vis (Figure 4.1), it is observed that absorption of PEDOT is very small in UV-Vis range, while the dye is responsible for most of the photo absorption with $\mathrm{PEDOT}^{+}$absorption seen mainly in near-IR region (Figure 4.2). The doping level of PEDOT increased with the increase amount of PEDOT in the photo-anode. The absorbance band around $850 \mathrm{~nm}$ and $2000 \mathrm{~nm}$ are associated with polarons [12]. In the doped PEDOT film, the absorbance around $850 \mathrm{~nm}$ decreases while around 2000 $\mathrm{nm}$ it increases. It has been earlier reported [12] the disappearing and decreasing of the absorbance band around $850 \mathrm{~nm}$ is due to the increase of the doping level and the formation of bipolaron [12]. It has also been observed that the polymerization charge increased with an increase of the absorption peak where the amount of PEDOT 
grown in photo-anode was increased. This is due to the majority of charge passed through the circuit during photo-electrochemical polymerization being consumed for PEDOT growth.

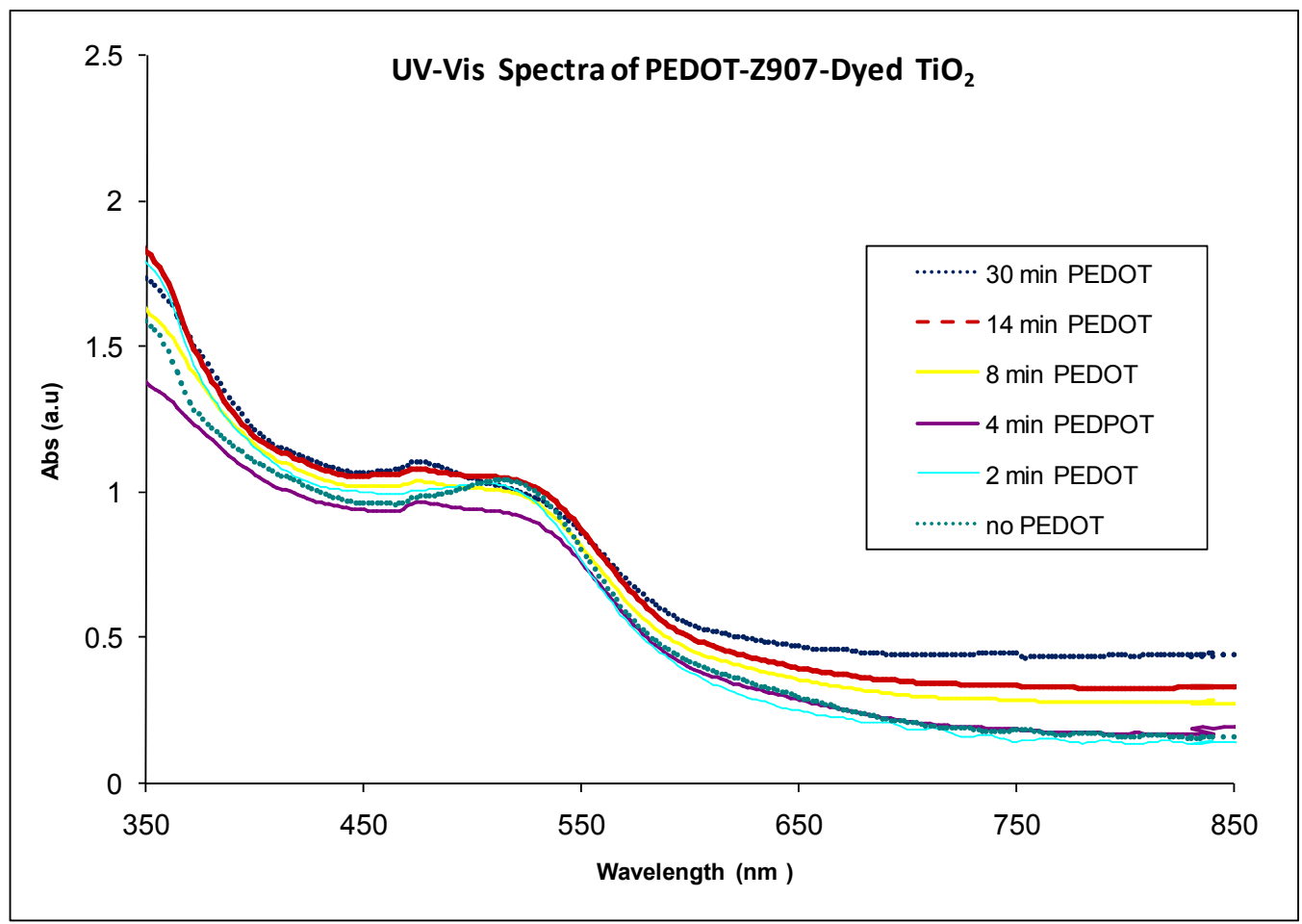

Figure 4.1: UV-Vis spectra of various photo-anode films prepared with different periods of growth of PEDOT. 


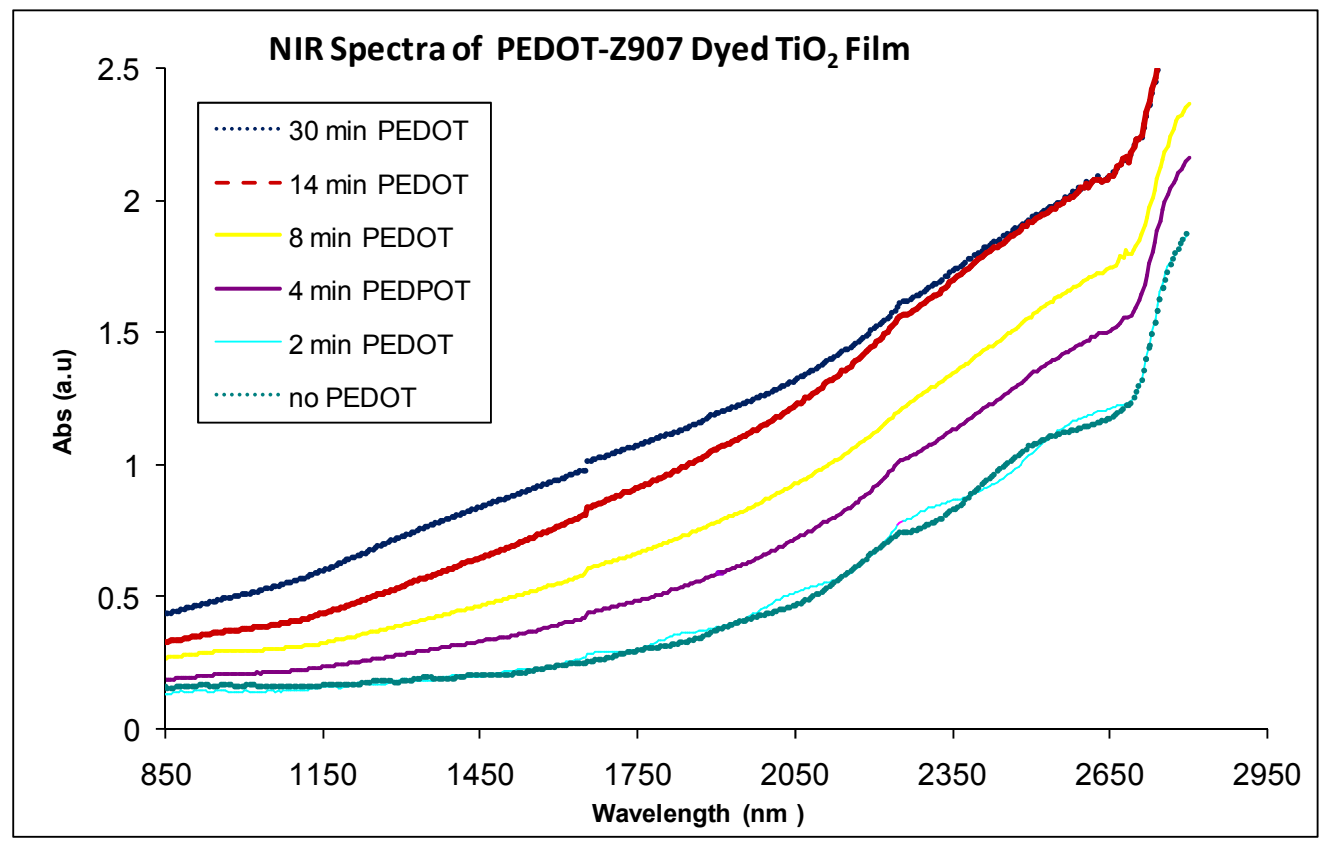

Figure 4.2: NIR spectra of various photo-anode films prepared with different periods of growth of PEDOT.

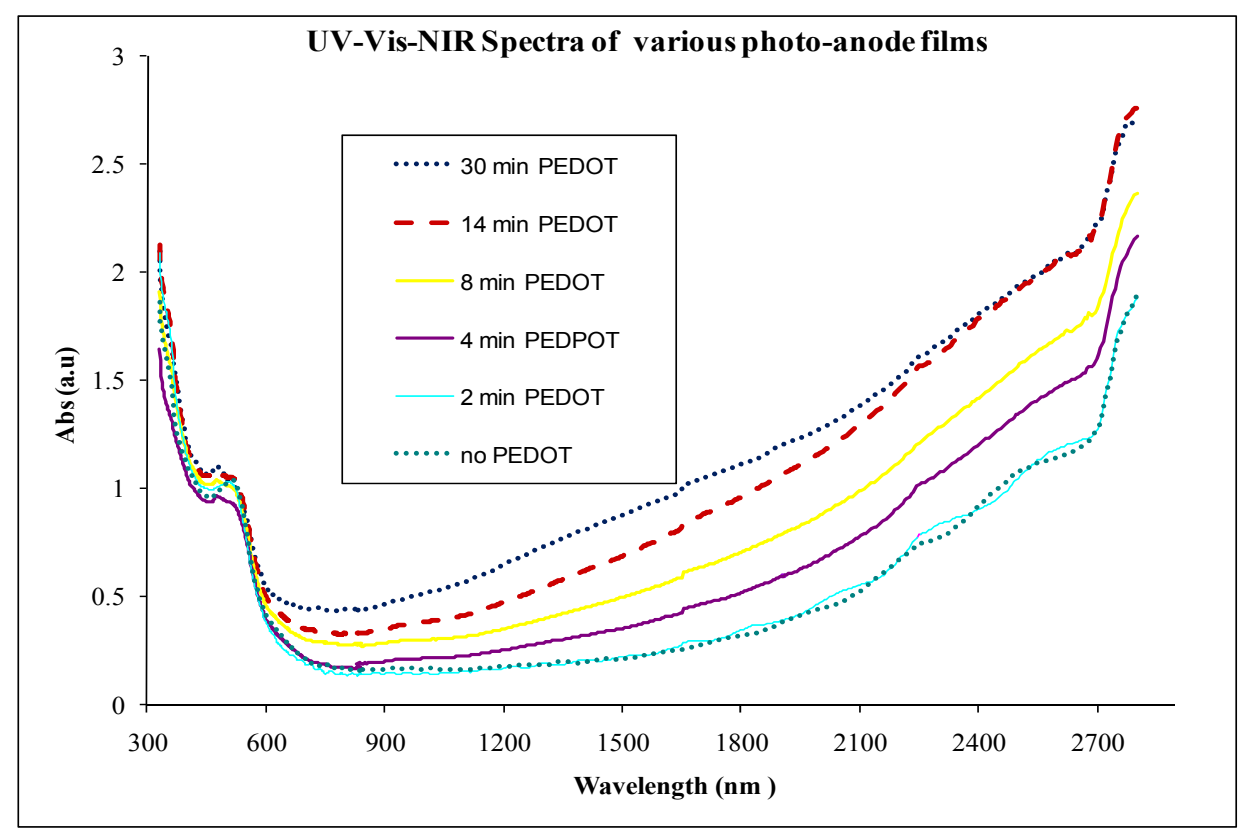

Figure 4.3: UV-Vis-NIR spectra of various photo-anode films prepared with different periods of growth of PEDOT. 


\subsubsection{Raman spectral studies}

The Raman spectra of each of the photo-anodes were also recorded using $785 \mathrm{~nm}$ excitation and shown in Figures 4.4, 4.5 and 4.6 respectively. The position of the symmetric $\mathrm{C}_{\alpha}=\mathrm{C}_{\beta}$ stretching band, associated with the vibration from both the neutral and oxidised states of PEDOT shifts when the ratio of the intensities of the vibrations of the two structures changes (Figure 4.6). The greater the shift of the symmetric $\mathrm{C}_{\alpha}=\mathrm{C}_{\beta}$ stretching band towards higher wave numbers, the greater the oxidation level of the PEDOT. The doping level of PEDOT with and without treatment with the ionic liquid (EMITFSI) can be estimated by substituting the ratio of the intensities of $\mathrm{C}_{\alpha}=\mathrm{C}_{\beta}$ the two vibrations in each case into a correlation equation which has been reported earlier [13], namely $I=0.087 y-2.279$, where $\mathrm{I}$ is the natural logarithm of the ratio and $\mathrm{y}$ is the percentage doping level in PEDOT. The doping levels of five PEDOT based photo-anodes samples prepared photo-electrochemically with a constant potential of $0.2 \mathrm{~V}$ (vs. $\mathrm{Ag} / \mathrm{AgCl}$ ), were calculated using the above equation. For comparison, Raman spectra of $\mathrm{TiO}_{2}$ film and $\mathrm{Z907}$ dye sensitized $\mathrm{TiO}_{2}$ film were also recorded.

The different types and quantities of the dopant in a conducting polymer can significantly affect its physical properties such as conductivity. The next part of this chapter reports the use of the various dopant anions during photo-polymerization and their effect on conductivity and performance of ssDSCs. It has been reported that the efficiency of the doping process depends on the nature of the dopant, such as its size, charge and the complexity of the dopant structure. In this chapter, only $\mathrm{ClO}_{4}{ }^{-}$as dopant ion has been used as this is the most efficient dopant because of its very small size and only a single negative charge. 


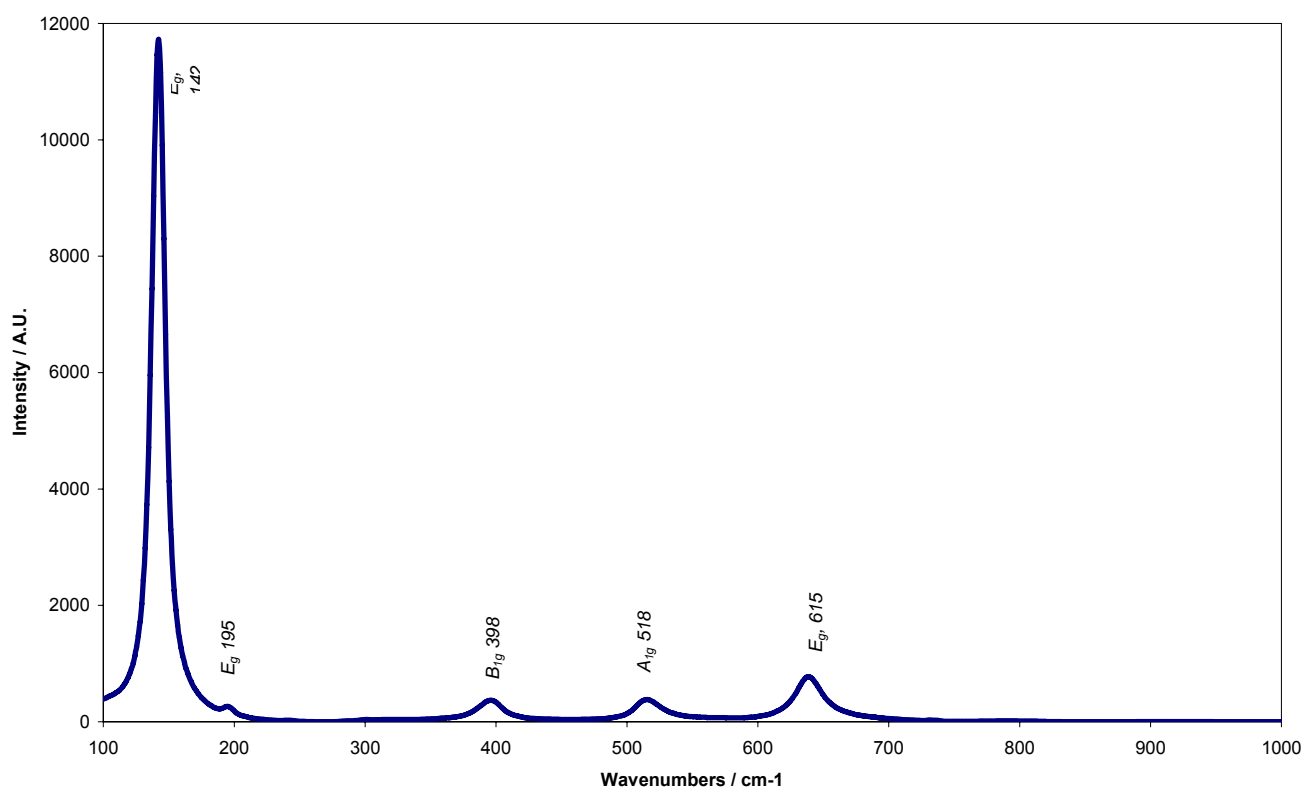

Figure 4.4: Raman spectrum of blank anatase $\mathrm{TiO}_{2}$

The Raman spectrum of the $\mathrm{TiO}_{2}$ blank (Figure 4.4) shows five distinctive characteristic peaks at $142,195,398,518$ and $615 \mathrm{~cm}^{-1}$, which belong to the five fundamental vibrational modes of the anatase $\mathrm{TiO}_{2}$ crystal phase (with $E_{g}, E_{g}, B_{l g}$, $A_{l g}$ and $E_{g}$ symmetries, respectively) [14-17]. However, for the Raman spectrum of dyed $\mathrm{TiO}_{2}$ (Figure 4.5), the ruthenium-based $\mathrm{Z907}$ dye cation on $\mathrm{TiO}_{2}$ bands are quite weak, when the PEDOT polymer was present. This is due to the much stronger PEDOT Raman bands. The normal Raman spectra of Ru-dyes $\mathrm{Z907}$ and $\mathrm{TiO}_{2}$ may not be properly acquired when using $785 \mathrm{~nm}$ excitation lasers because of the strong fluorescence that overwhelms the Raman transition [17]. 


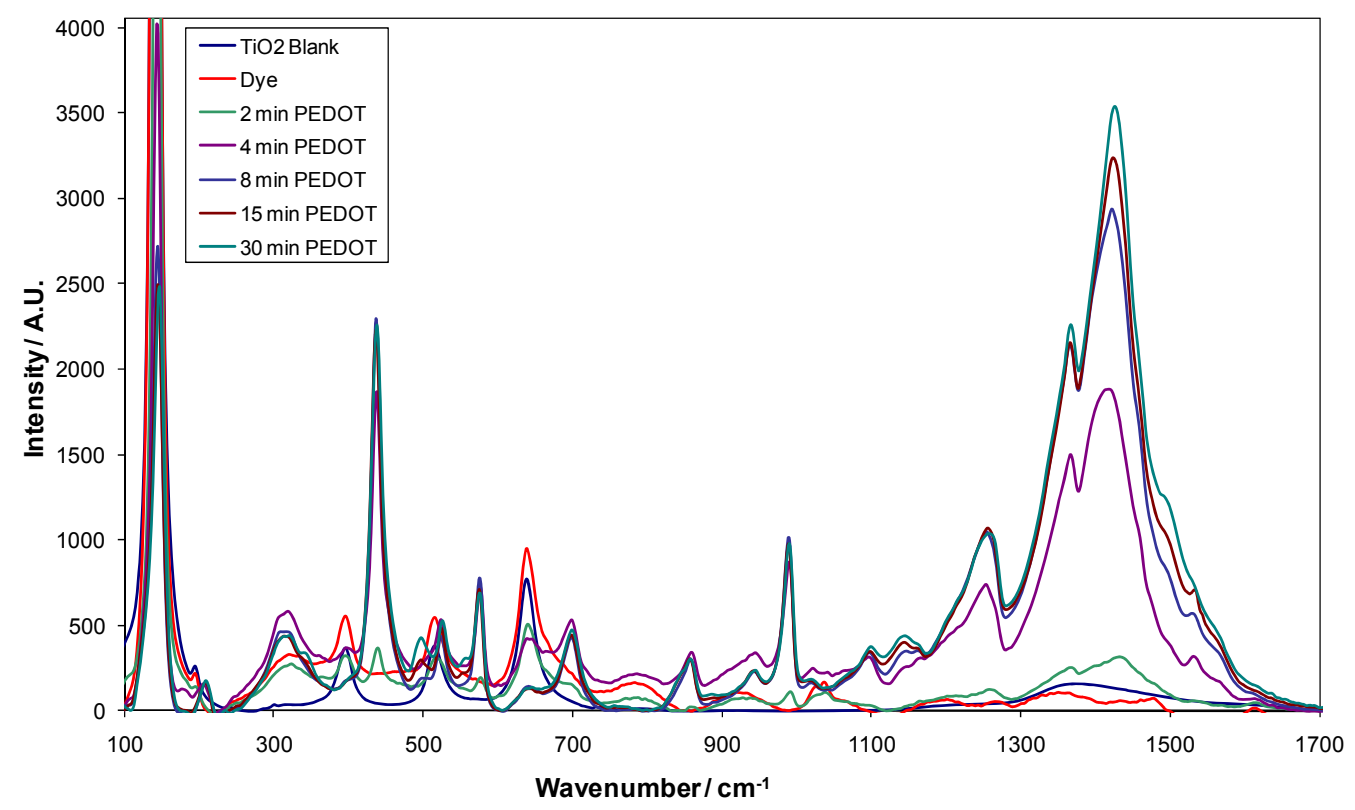

Figure 4.5: Raman spectra of photo-anodes in which PEDOT has been deposited over various lengths of time (without ionic liquid surface treatment).

As deposition time increases, there is a significant increase in the intensity of the bands relating to the conducting polymer around $1421 \mathrm{~cm}^{-1}$ (Figure 4.5). The spectra are typical of PEDOT in the oxidised state. Most notable are the $\mathrm{C} \alpha=\mathrm{C}_{\beta}$ at $1425 \mathrm{~cm}^{-1}$ (increase in the quinoid structure) and the $C_{\beta}-C_{\beta}$ at $1369 \mathrm{~cm}^{-1}$ (quinoid dications), and the $\mathrm{C}_{\alpha}=\mathrm{C}_{\beta}{ }^{\prime}$ stretchings [17]. The main peak is around $1423 \mathrm{~cm}^{-1}$ is due to the $\mathrm{C}=\mathrm{C}$ symmetrical stretching vibrations [17]. The peaks centered at 1520 and $1570 \mathrm{~cm}^{-1}$ are associated with the $\mathrm{C}=\mathrm{C}$ asymmetric stretching vibration that correspond to thiophene rings in the middle and at the end of the chains, respectively [17]. There is also a decrease in the intensity of the bands assigned to $\mathrm{TiO}_{2}$ and the ruthenium $\mathrm{Z907}$ dye cation with an increase in the amount of PEDOT. The various representative PEDOT polymer Raman bands are presented in Table 4.1 and quite similar to the earlier reported [12, 18-19]. 


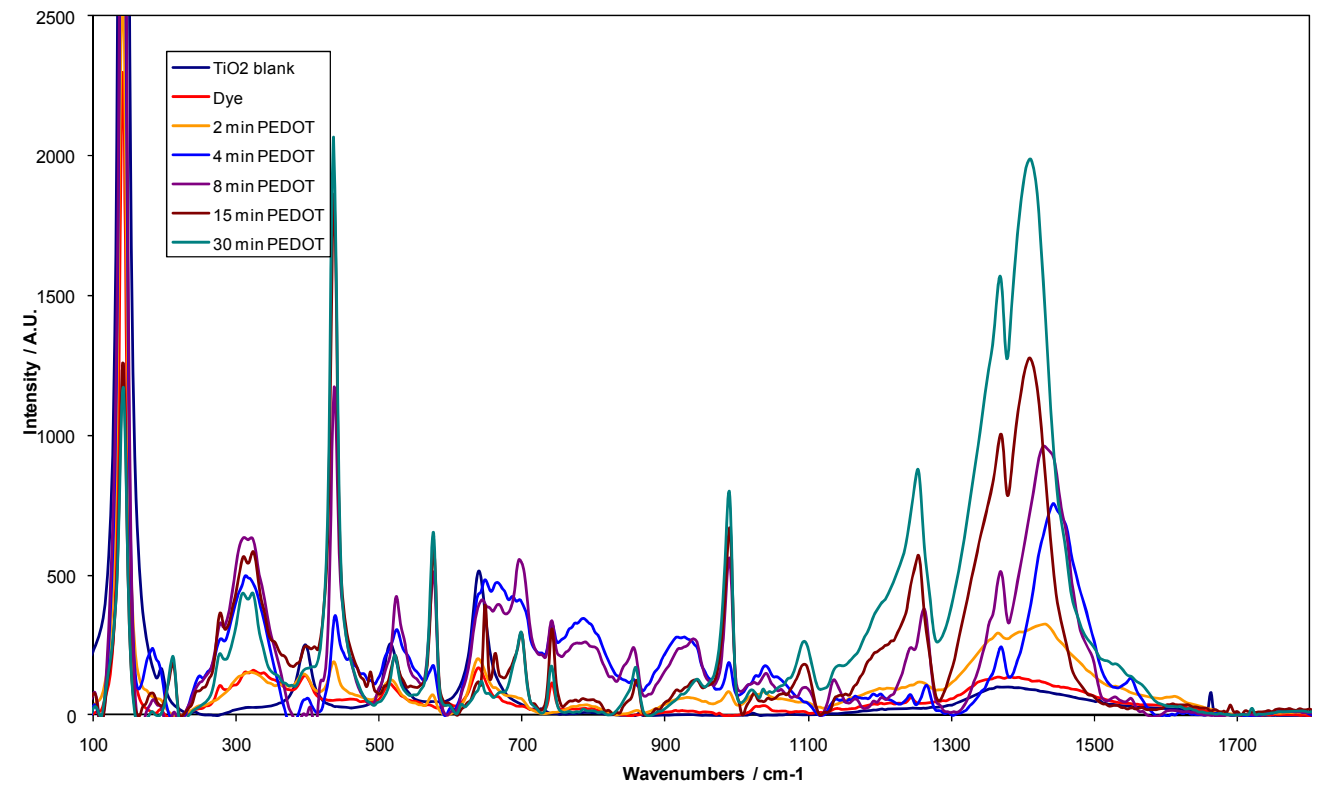

Figure 4.6: Raman spectra of photo-anodes in which PEDOT has been deposited over various lengths of time (with ionic liquid surface treatment).

Table 4.1: Polymer Raman bands assignments:

\begin{tabular}{lc}
\hline \multicolumn{1}{c}{ Assignment } & Polymer $\left(\mathrm{cm}^{-1}\right)$ \\
\hline $\mathrm{C}=\mathrm{C}$ ring stretching (anti) & 1540 \\
$\mathrm{C}=\mathrm{C}$ ring stretching & 1497 \\
$\mathrm{C}=\mathrm{C}$ ring stretching & 1425 \\
$\mathrm{C}-\mathrm{C}$ ring stretching & 1369 \\
$\mathrm{C}-\mathrm{C}$ stretching & 1260 \\
$\mathrm{C}-\mathrm{H}$ bending & 1145 \\
$\mathrm{C}-\mathrm{C}$ anti stretching & 1104 \\
$\mathrm{CH} \mathrm{H}_{3}$ ring stretching & 990 \\
$\mathrm{C}-\mathrm{S}$ ring stretching & 860 \\
$\mathrm{C}-\mathrm{S}-\mathrm{C}$ ring deformation & 700 \\
ring in-plane bending & 527 \\
\hline
\end{tabular}


The effect of treating the photo-anodes with the ionic liquid EMITFSI was also examined. The Raman spectrum of PEDOT/Z907/ $\mathrm{TiO}_{2}$ photo-anode is shown in Figure 4.7, and the wavenumbers of the observed bands listed in Table 4.1. Also of note is the fact that for the thinner PEDOT films there is a shift to higher wave numbers for the $\mathrm{C}=\mathrm{C}$ stretching band, although this reverts back to the norm for films in which the PEDOT was grown for 2 minutes and 4 minutes.

Treatment of the photo-anodes with the ionic liquid is seen to cause little change in the PEDOT $\mathrm{C} \alpha=\mathrm{C}_{\beta}$ bands.

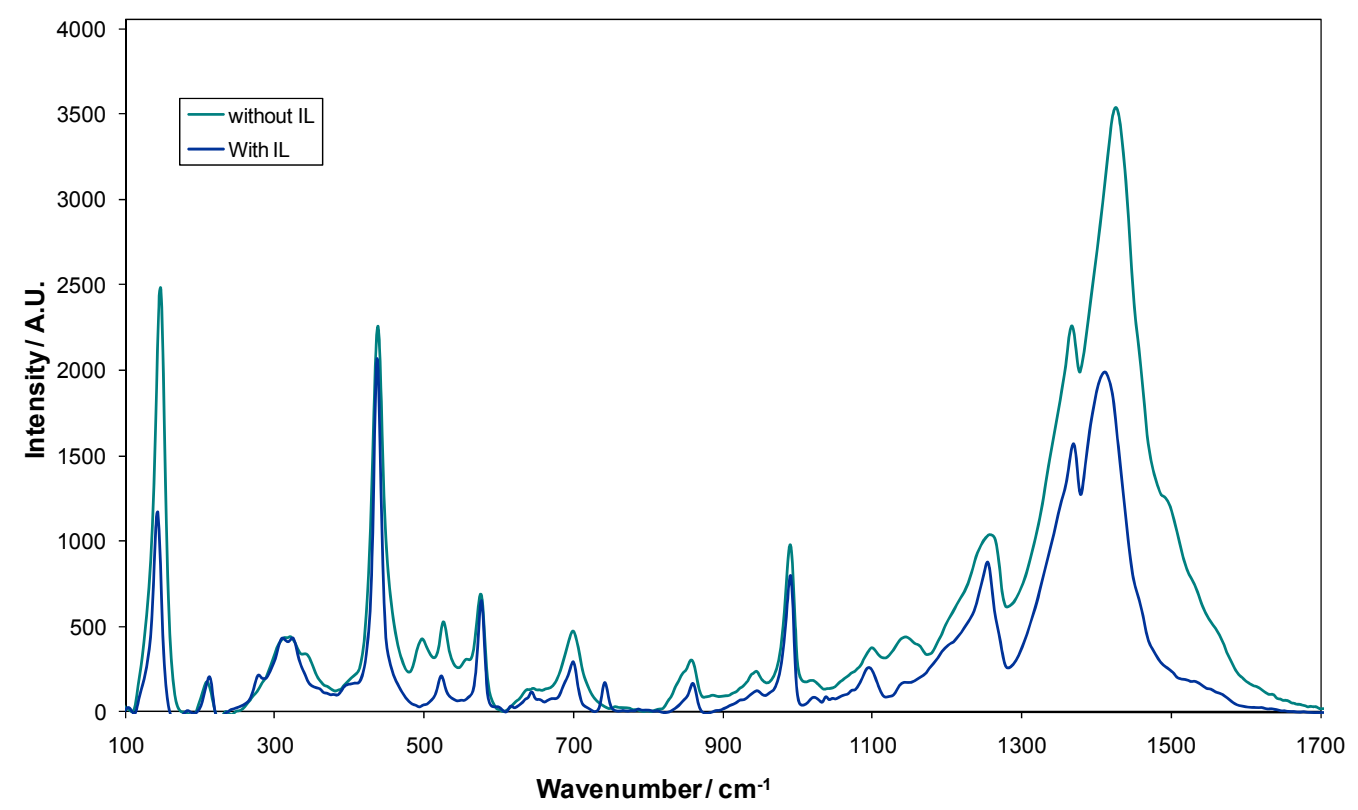

Figure 4.7: Raman spectra of two photo-anodes where one photo-anode was treated with ionic liquid (IL) and one without IL (in both cases PEDOT has been deposited over 30 minutes).

\subsubsection{MALDI (ToF) mass spectra}

Matrix assisted laser desorption ionisation (MALDI) coupled with the Time-ofFlight (ToF) technique have been recently employed to analyze conducting polymers [8]. In the present work, the mass spectrum generated can give information about repeating chain units, end groups, and the molecular weight distribution of the 
PEDOT. The amount of PEDOT depends on the intensity and the wavelength of the light which is irradiated on the anode side and thickness of the $\mathrm{TiO}_{2}$ film. It was found that the longer the PEDOT deposition time, the longer the conjugation length of the bis-EDOT unit. From Figure 4.8, where 30 minutes PEDOT growth was used, the longest PEDOT polymer consisted of 19 bis-EDOT repeating units, whereas after 2 minutes of PEDOT, the longest polymer unit had 5 bis-EDOT units. It can therefore be concluded that with longer the polymer growth time, the more PEDOT was grown into the nano-matrix of dyed $\mathrm{TiO}_{2}$.

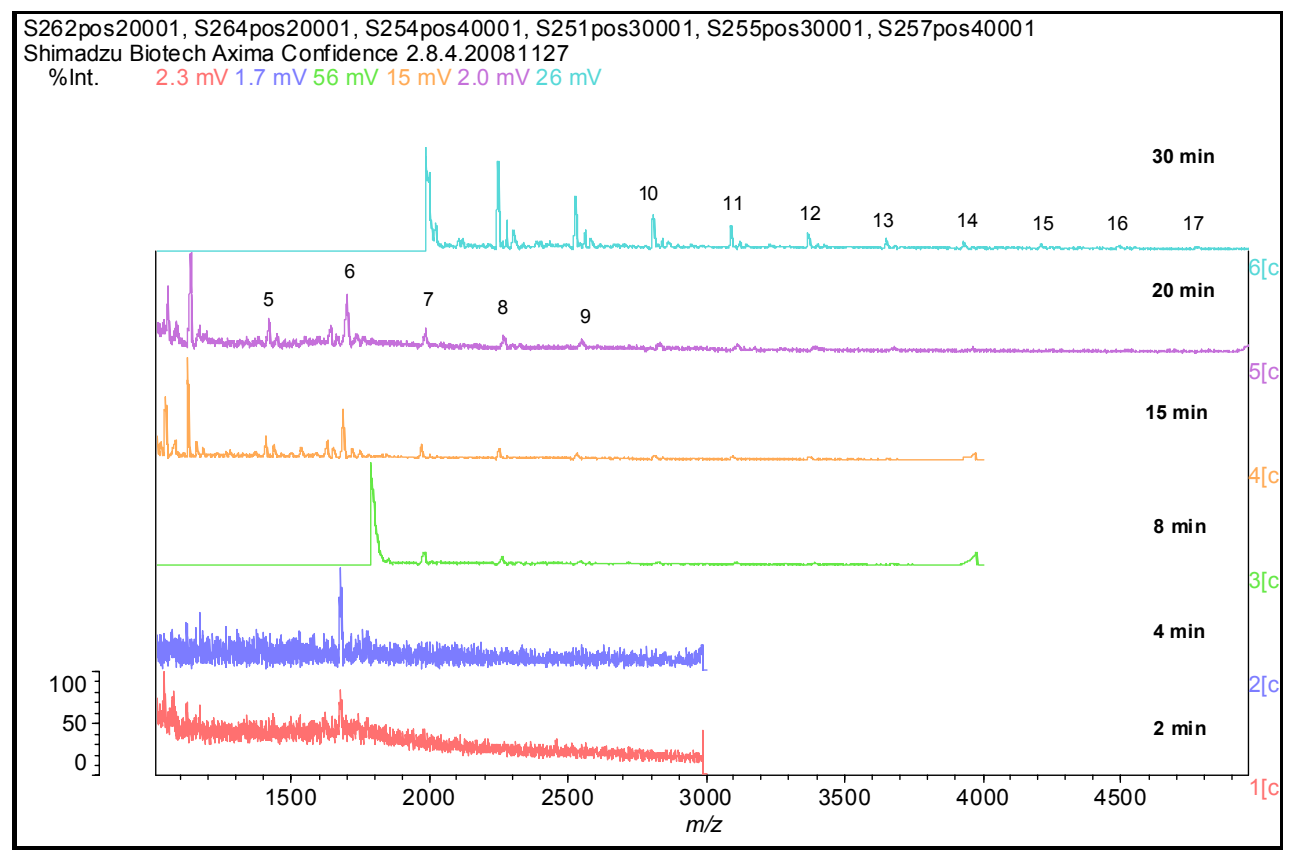

Figure 4.8: MALDI mass spectrum of various photo-anodes where PEDOT was grown over different periods.

\subsubsection{Morphology studies by SEM}

SEM images of the surface and cross-sectional morphology of the various PEDOT grown on $\mathrm{Z907}$ dyed $\mathrm{TiO}_{2}$ are presented here (Figures 4.9-4.14). Photo-anodes showed that increased PEDOT growth time generates larger size particles (Figure 4.11 and Figure 4.13). This results in better photovoltaic performance. The structure of the PEDOT film deposited by photo-electrochemical polymerization into the dyed 
$\mathrm{TiO}_{2}$ nano-matrix of the photo-anode was also investigated via SEM, as what that of the original $\mathrm{Z} 907$ dyed $\mathrm{TiO}_{2}$ structure without PEDOT. Figure 4.9 presents the cross-sectional SEM image of dyed $\mathrm{TiO}_{2}$ (left) and an enlarged cross-sectional morphology image of the left image (right). The various amount of PEDOT growth on the photo-anodes and their morphology are presented in Figures 4.10-4.14. These cross-sectional and surface morphology studies show that PEDOT films grown for longer times possess fewer pores and give better coverage of the $\mathrm{Z} 907$ dyed $\mathrm{TiO}_{2}$. With less deposited PEDOT, the dyed $\mathrm{TiO}_{2}$ is less well covered by the polymer and exhibits core-like structure (Figure 4.14).

The less non-porous photo-anode which was covered by PEDOT was also investigated to determine the smoothness of the deposited films. How the photopolymerized electrode influenced the photovoltaic performances of respective devices is presented in this chapter.
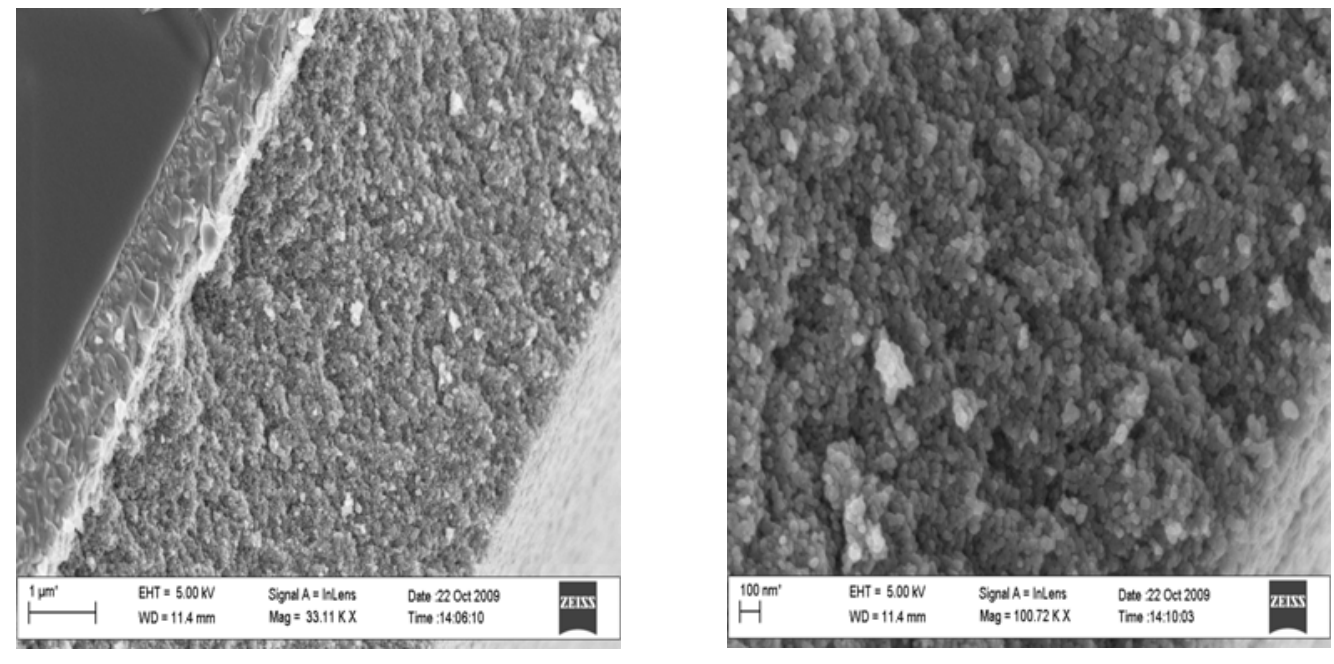

Figure 4.9: (Left) Cross-sectional SEM image of $\mathrm{Z} 907$ dyed $\mathrm{TiO}_{2}$ (no PEDOT deposited) and (Right) an enlarged cross-sectional morphology image. 

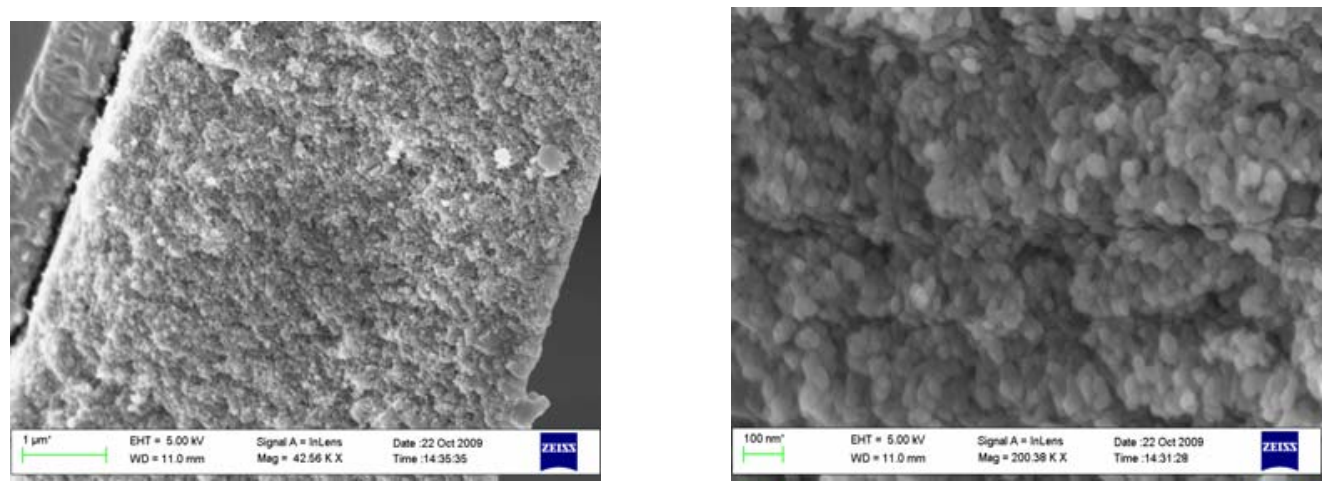

Figure 4.10: Cross-sectional SEM image of a photo-anode where PEDOT was grown for 2 minutes (left) and enlarged image (right).
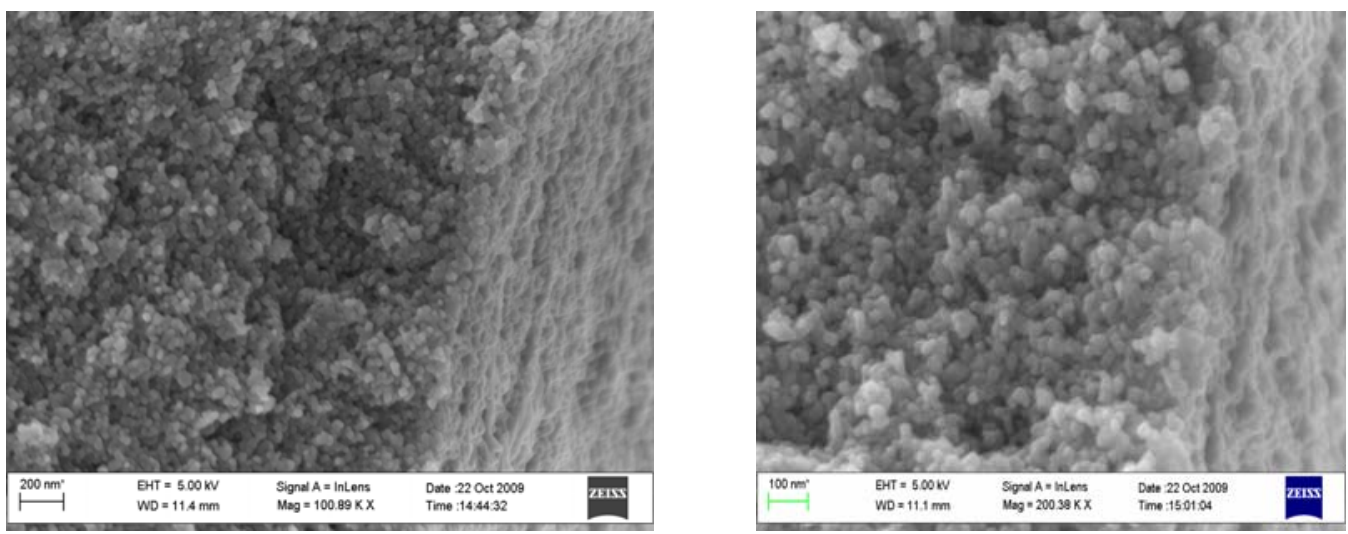

Figure 4.11: Cross-sectional SEM image of a photo-anode in which PEDOT was grown for 4 minutes (left) and 8 minutes (right).

It shows from SEM (Figure 4.14) images that the increased PEDOT growth time generated larger sized particles, particles means here PEDOT coated dyed $\mathrm{TiO}_{2}$. The better coverage of the $\mathrm{TiO}_{2}$ by the PEDOT with increased growth time is evident only on the surface of the photo-anode film. An apparent difference in the PEDOT coverage between the surface and the cross sections of the anodes may be due to the discontinuous surface PEDOT layer formed. Moreover the thickness of PEDOT is not equal the thickness of PEDOT within the nanomatrix dyed $\mathrm{TiO}_{2}$. 

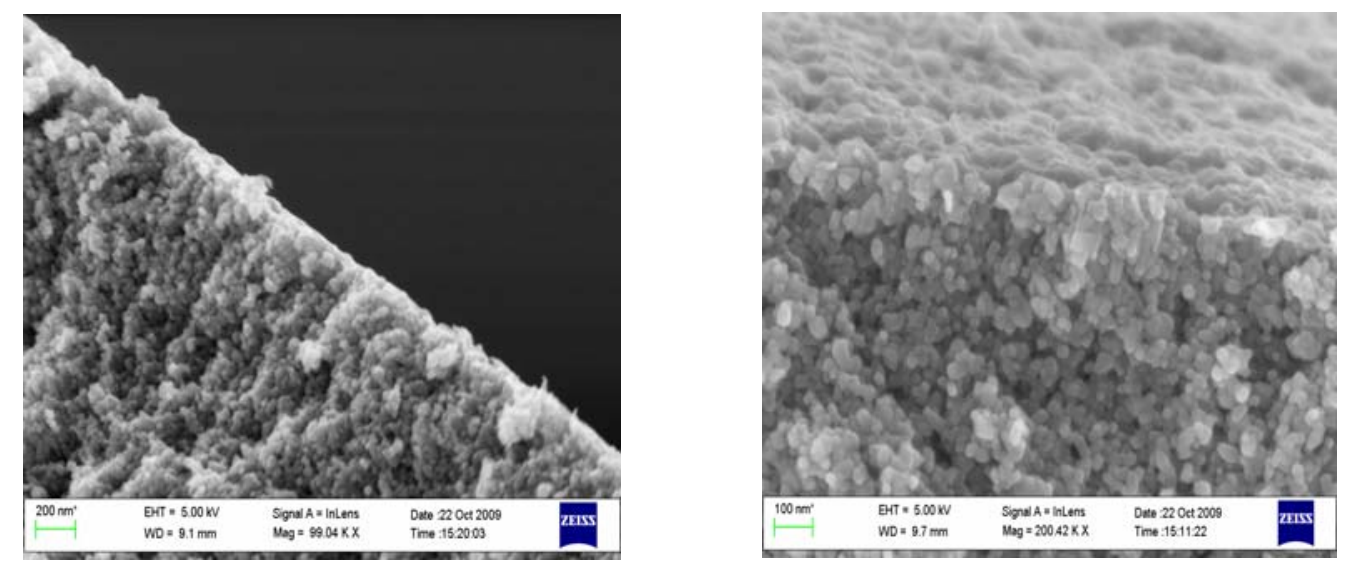

Figure 4.12: Cross-sectional SEM image of a photo-anode where PEDOT was grown for 15 minutes (left) and enlarged image (right).
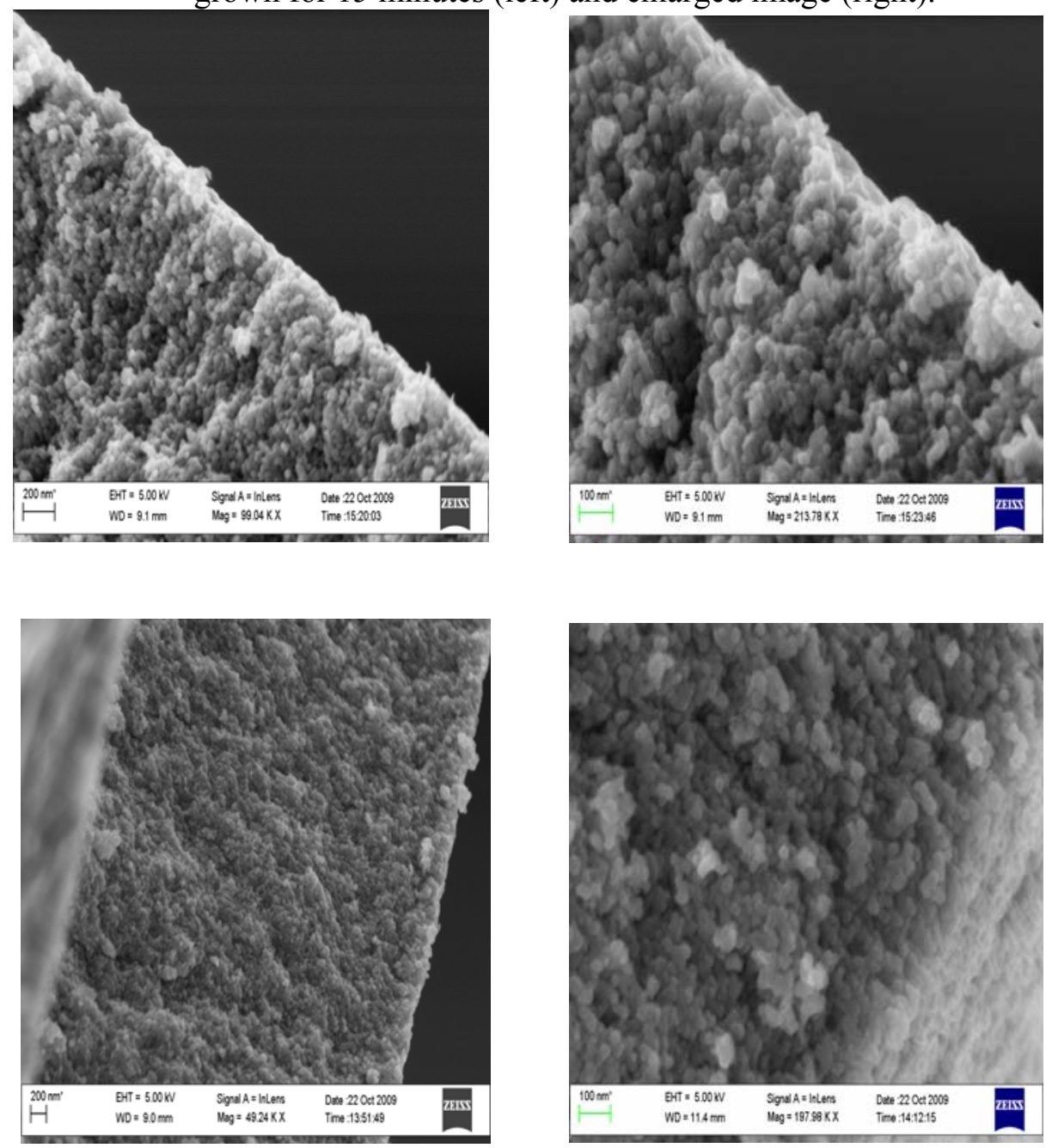

Figure 4.13: Cross-sectional SEM image of a photo-anode where PEDOT was grown for 30 minutes with four different magnifications. 

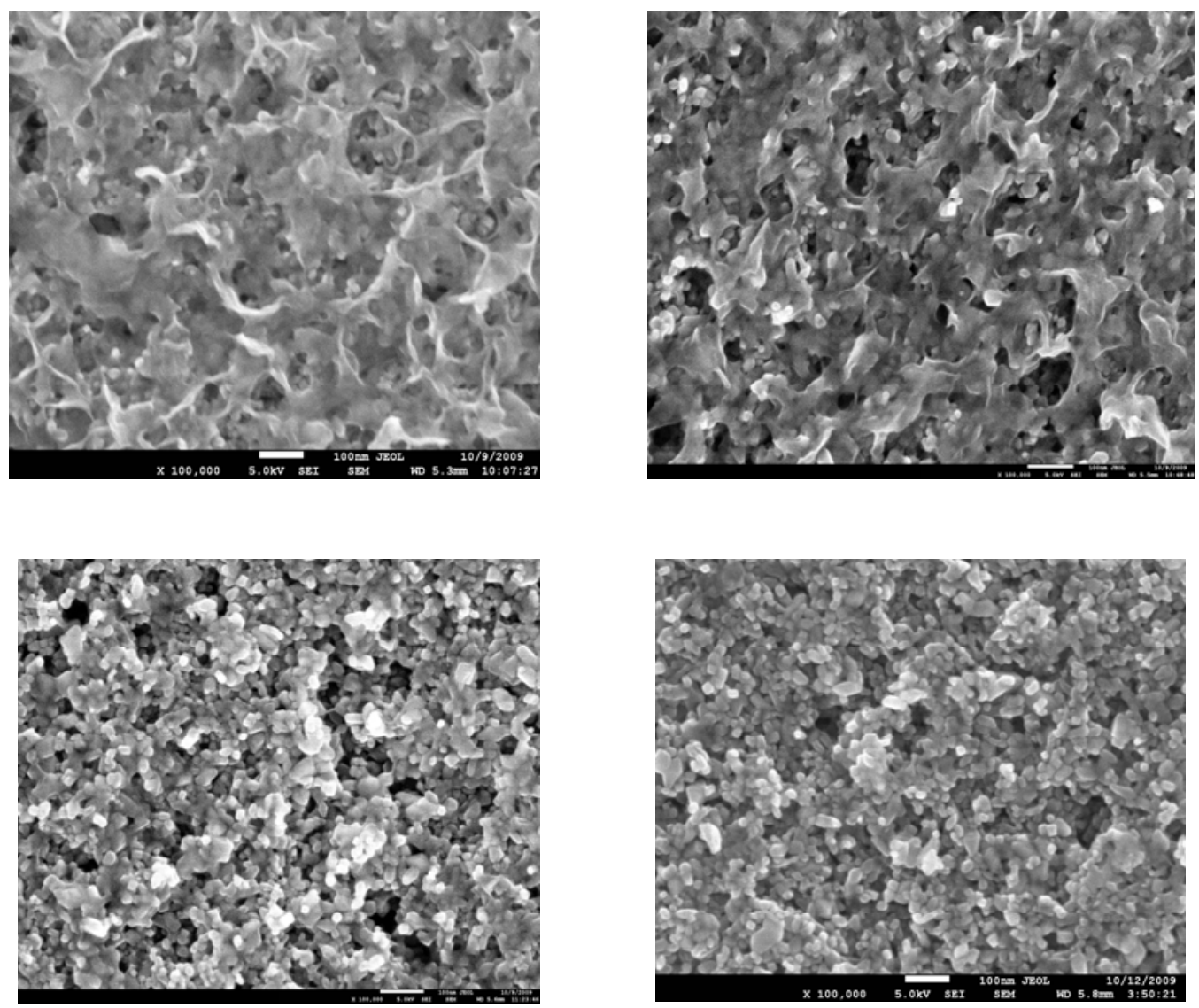

Figure 4.14: SEM image of surface morphology of a photo-anodes where PEDOT was grown for 30 minutes (top left) and $15 \mathrm{~min}$ (top right) and 8 minutes (bottom left) and 4 minutes (bottom right).

\subsubsection{SEM-EDX analysis:}

SEM-EDX analysis was carried out on the surface and cross-sectional areas of PEDOT deposited for varying periods of time on the photo-anodes. It was observed that samples of PEDOT that were deposited for 30 minutes showed higher sulphur content compared to samples of PEDOT deposited for less time. This corresponds well with the above observation that an increase of growth time generates PEDOT with larger particles, and perhaps is the reason for the better PV performance. Analysis was done on four separate $4 \times 50 \mu \mathrm{m}^{2}$ areas on each sample to confirm the uniformity of PEDOT distribution. Figure 4.15 shows the averaged SEM-EDX analysis spectrum for a photo-anode in which the PEDOT was deposited for 30 
minutes. For comparisons, a photo-anode where PEDOT was grown for 8 minutes is presented in Figure 4.16.
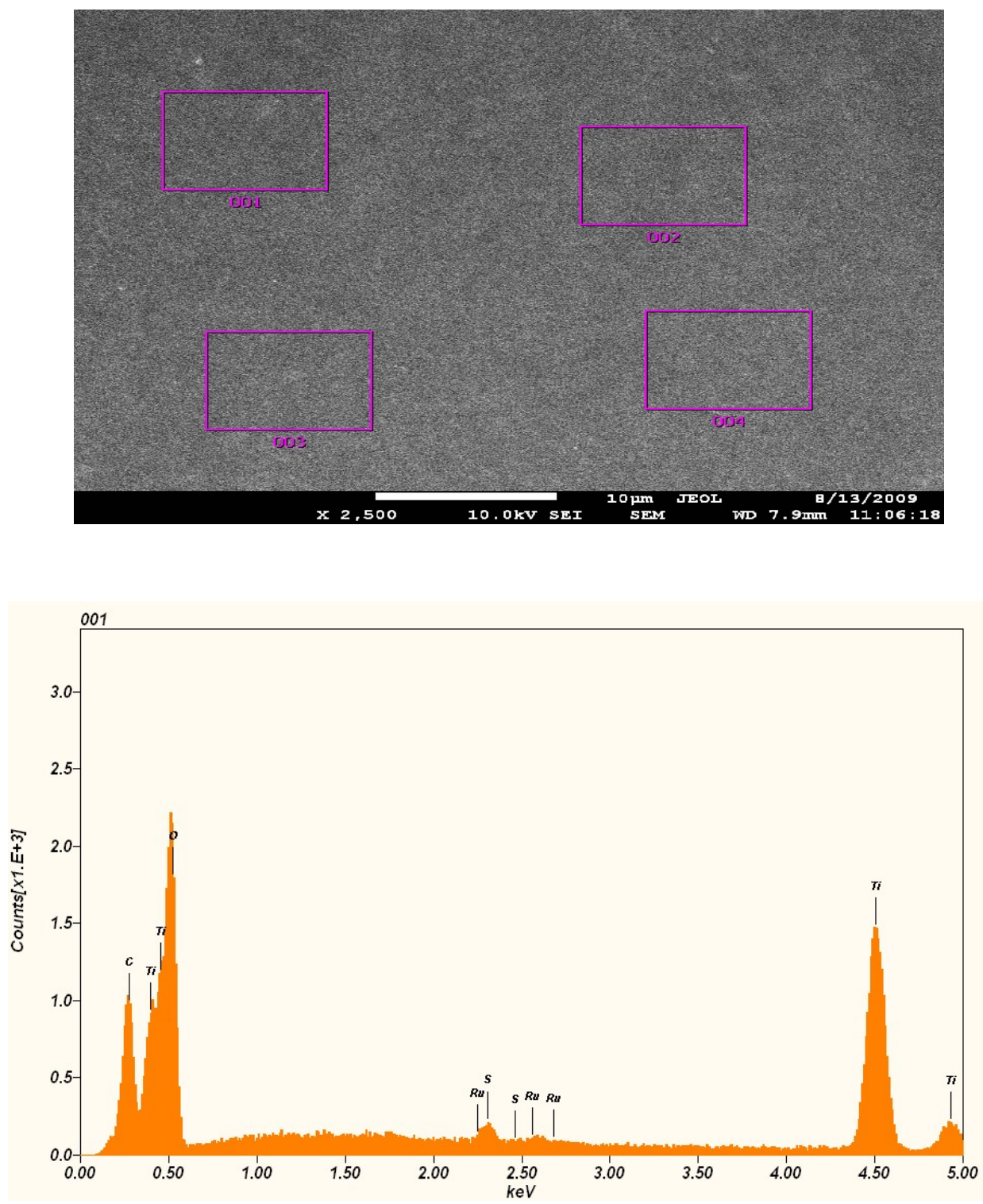

Figure 4.15: The averaged SEM-EDXA spectrum for a photo-anode in which PEDOT was grown for 30 minutes (Averaged for four separate $4 \times 50$ $\mu \mathrm{m}^{2}$ areas). 

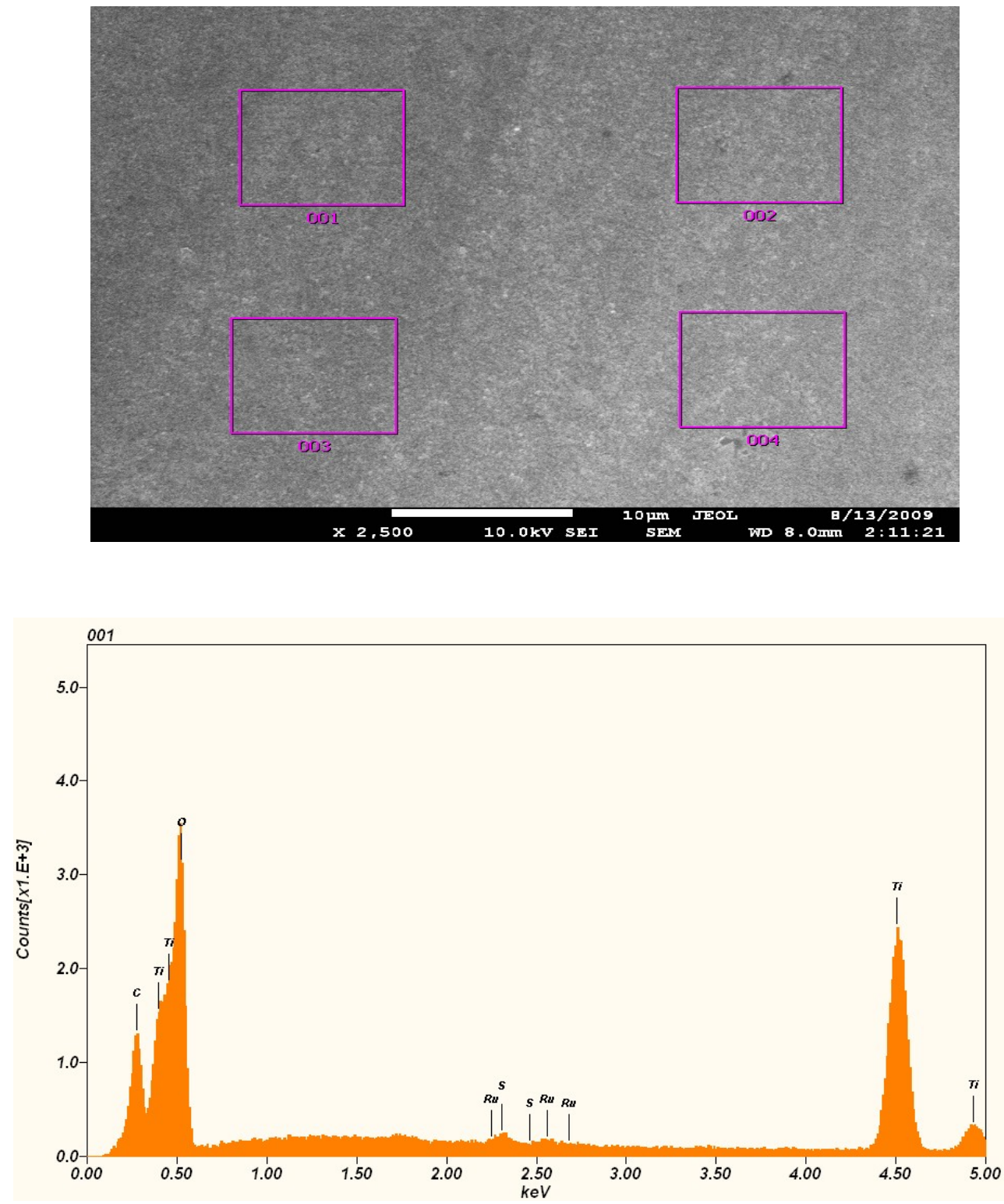

Figure 4.16: The averaged SEM-EDXA spectrum for a photo-anode in which PEDOT was grown for 8 minutes (Averaged for four separate $4 \times 50$ $\mu \mathrm{m}^{2}$ areas).

\subsubsection{Morphology studies by AFM}

The AFM surface topography images were also obtained in order to confirm the surface morphology recorded using SEM. The comparisons were made by firstly 
imaging the material surface using AFM, then immediately imaging the same area of the film using SEM. AFM images of various layers of the photo-anode, namely the FTO layer, blocking $\mathrm{TiO}_{2}$ layer, mesoporous $\mathrm{TiO}_{2}$ layer, dyed $\mathrm{TiO}_{2}$ layer are presented in Figures 4.17 (a, b, c, d). Figures 4.17 (a, b, c, d) the AFM images of FTO glass, FTO_TiO $2(\sim 5 \mathrm{~nm}$ particle size $), \mathrm{FTO}_{-} \mathrm{TiO}_{2}(\sim 30-40 \mathrm{~nm}$ particle size $)$, FTO_dyed $\mathrm{TiO}_{2}$, while Figures $4.18(\mathrm{a}, \mathrm{b})$ and Figures 4.19 (a, b, c) show five topography images of photo-anode films where different amounts of PEDOT were grown over time. Figure 4.19 reveals the surface topography of the photo-anode, upon which PEDOT had been grown for 8, 15, and 30 minutes, respectively. The films exhibited a generally rough morphology, with some features protruding up to $40 \mathrm{~nm}$ from the base film surface. In Figure 4.19, upon imaging at a higher magnification $(2-2.5 \mu \mathrm{m})$, it was found that the higher regions (lighter coloured areas) were more porous compared to the lower regions of the film. This indicates that more dyed $\mathrm{TiO}_{2}$ nano-particles were encapsulated or may be coated with PEDOT.
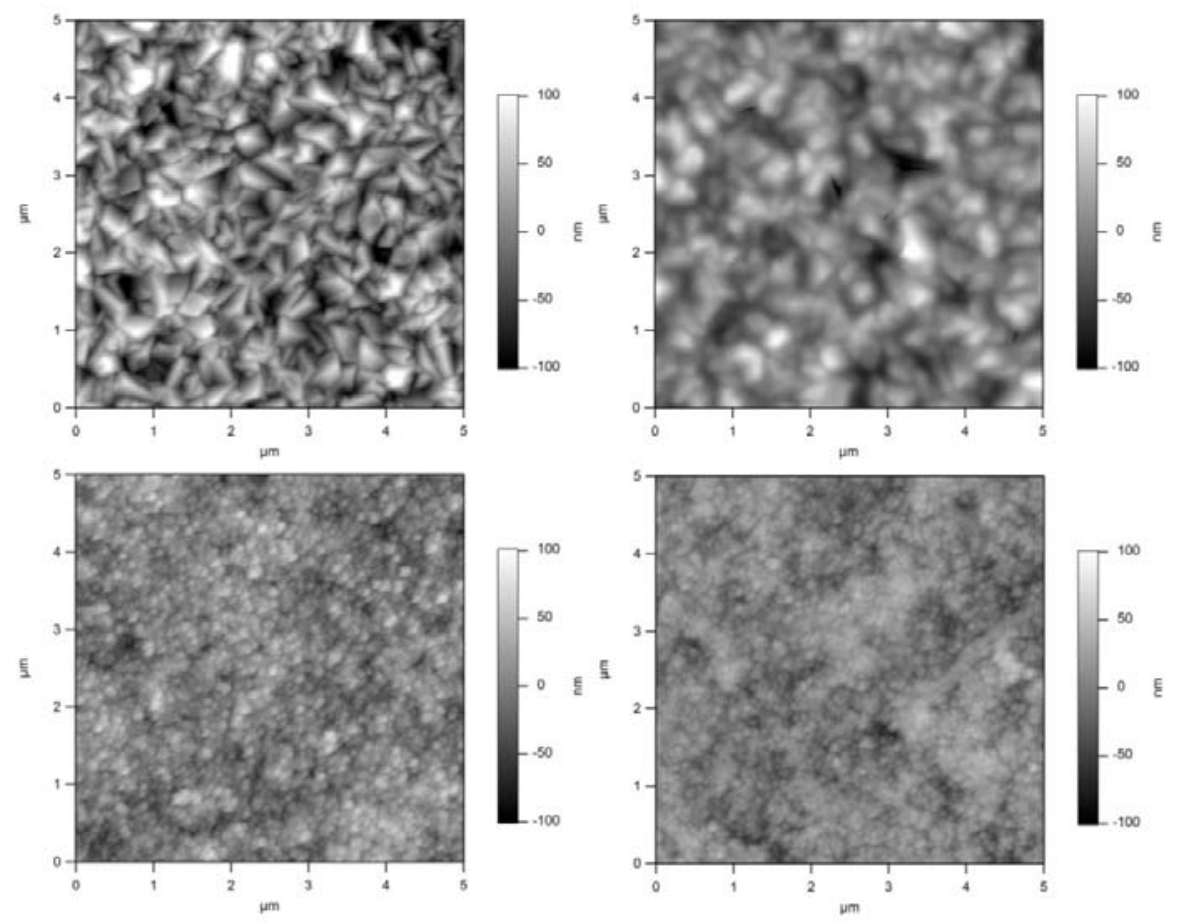

Figure 4.17: AFM images of FTO layer (a), blocking $\mathrm{TiO}_{2}$ layer (b), mesoporous $\mathrm{TiO}_{2}$ layer (c), and dyed $\mathrm{TiO}_{2}$ layer (d). 
Images were therefore recorded for several samples representing PEDOT grown under a range of conditions (e.g. 2-30 minutes growth time). However, it was observed that PEDOT samples grown for longer periods caused fouling of the cantilever tip with PEDOT from the photo-anode, thus making imaging difficult and affecting image quality.

In the case of 30 min PEDOT growth, a higher surface roughness was achieved. This permits better matching with the roughness of the Goretex ${ }^{\circledR}-A u-P E D O T$ counter electrode, resulting in good clipping in assembly of the ssDSC and consequently better PV performance. All photo-anode images were mapped using a micrograph range of 2-2.5 $\mu \mathrm{m}$. Figure 4.20 shows comparisons of the relative surface roughness of five different photo-anodes (where the time of PEDOT growth was varied), along with the surface roughness of different layers of photo-anodes. When more PEDOT was grown (30 min), the area (red colour) in the histogram became broader, without PEDOT (pink area), the photo-anode became smoother.
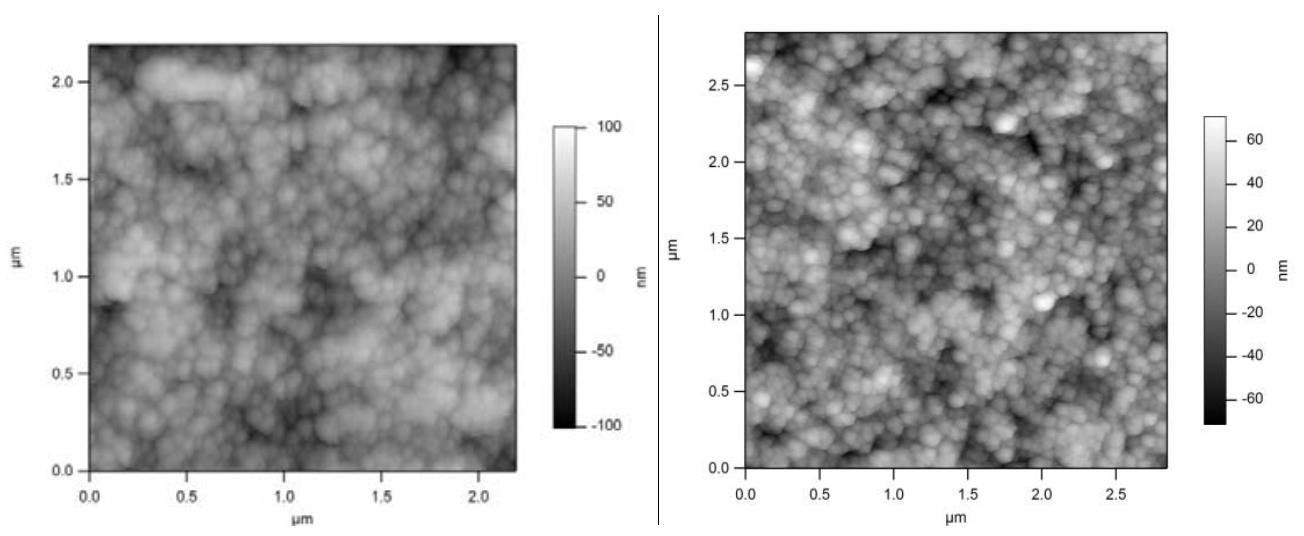

Figure 4.18: AFM images of PEDOT grown for 2 minutes (a) and 4 minutes (b) into the photo-anodes. 

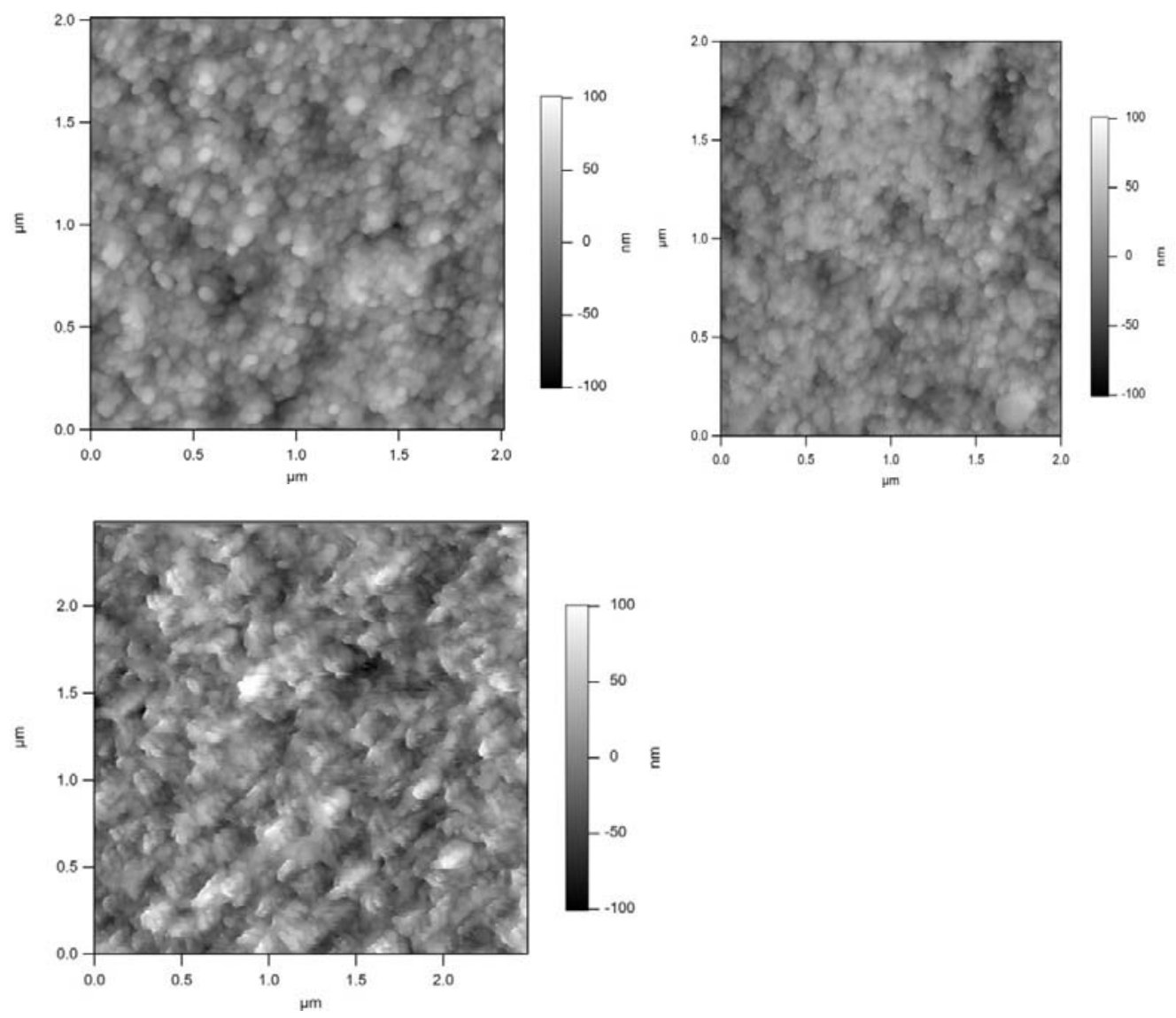

Figure: 4.19: AFM images of PEDOT grown for (a) 8 minutes, (b) 15 minutes, and (c) 30 minutes into the photo-anode. 


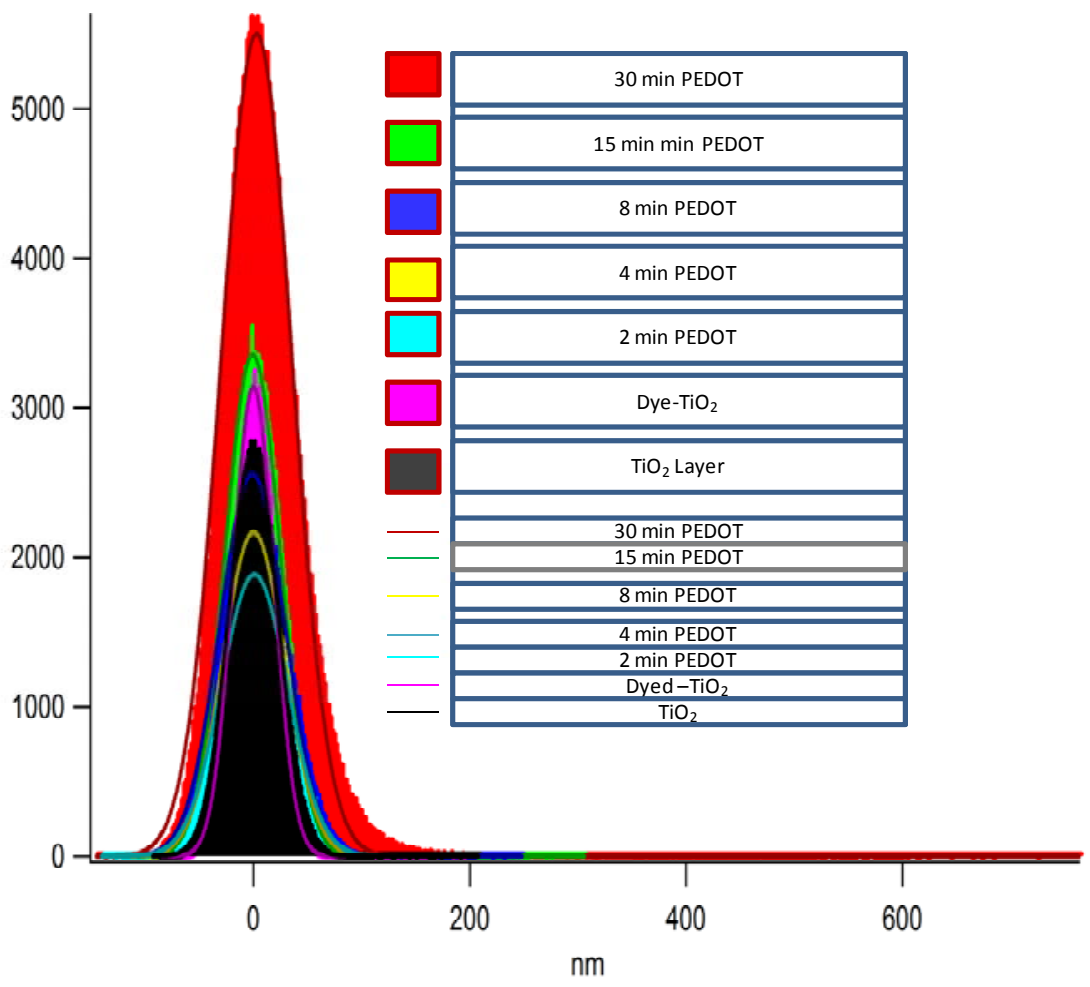

Figure 4.20: The roughness histograms of different layers of photo-anodes in which PEDOT was grown for various periods.

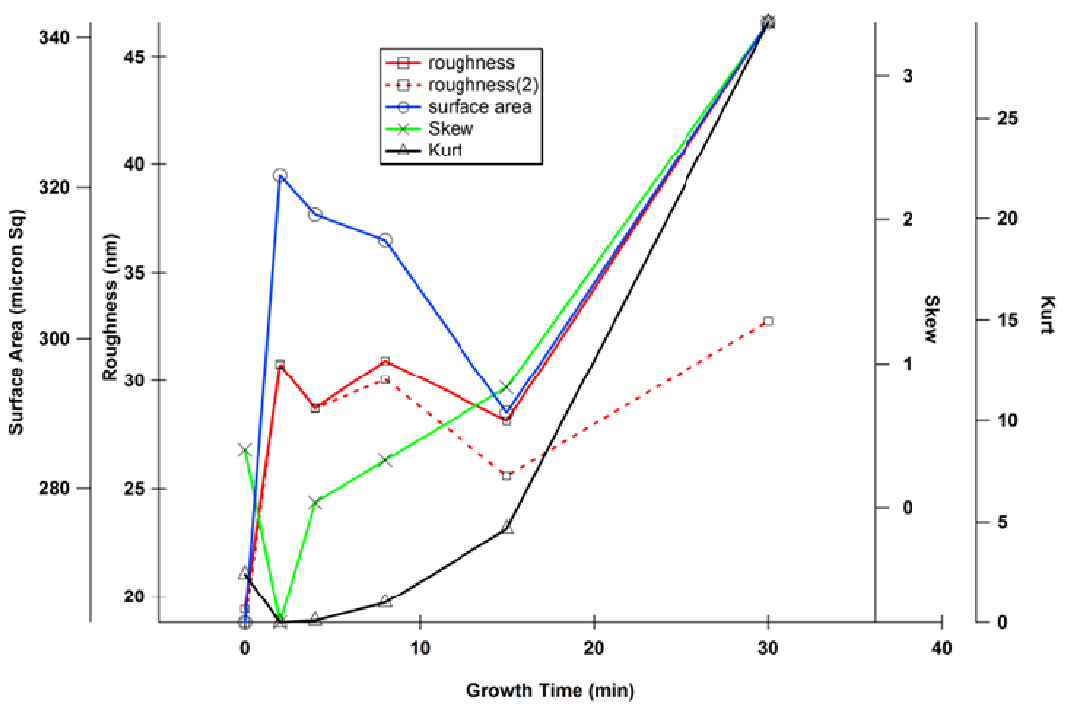

Figure 4.21: Quantitative analysis of topographical properties of PEDOT.

From Figure 4.21, it is observed that both the roughness and surface area increase upon deposition of PEDOT on the $\mathrm{Z907} / \mathrm{TiO}_{2}$ for 2 minutes. Where PEDOT was 
grown for longer periods (2-15 minutes) the roughness and surface area plateaus, and then increased further for the case of 30 minutes deposition.

Negative skew in Figure 4.21 indicates valleys, while positive skew indicates peaks. The skew initially decreases after deposition at 2 minutes, most probably because the PEDOT hasn't filled in, followed by a linear increase associated with the nodularpeak morphology of the PEDOT.

The Kurt parameter describes the proportion of surface that falls within a height range. For uniform surfaces, Kurt $=3$; higher aspect ratio $<3$ (e.g. high proportion of the surface falls within a narrow height range); and lower aspect ratio $>3$. At initial 2 minute deposition the surface features have higher aspect ratio values, more spikes, then the Kurt increases non-linearly with increased growth time and eventually reaches above 3 (again as a nodular morphology develops).

It has been observed that both from SEM and AFM images there remains many pores that are not filled up by PEDOT. To improve the solid-state DSCs performance further, the following must be achieved:

(i) Better pore filling by PEDOT into the dyed $\mathrm{TiO}_{2}$ nanomatrix with different morphology of $\mathrm{TiO}_{2}$ (varying the pore size, $\mathrm{TiO}_{2}$ particle size, size distribution, film thickness).

(ii) Higher mobility of hole transporting material leading to reduced charge recombination

(iii) A more negative HOMO level in the HTM will increase the $V_{\text {oc }}$.

(iv) Better electronic contact between $\mathrm{HTM}$, dye and $\mathrm{TiO}_{2}$.

Photo-anode schematics are given in Figure 4.22. 


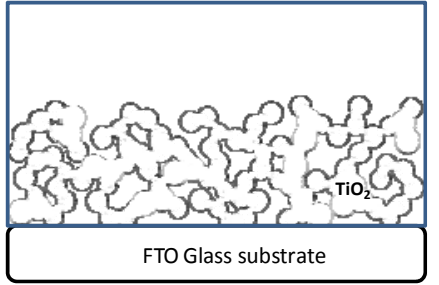

Figure A: Schematic of $\mathrm{TiO}_{2}$ on FTO substrate.

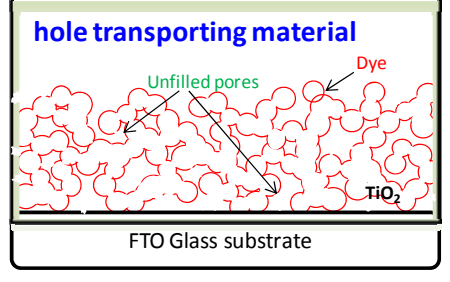

Figure C: Schematic of photo-anode with the pores in the dyed $\mathrm{TiO}_{2}$ nanomatrix are partially filled by hole transporting material (PEDOT).

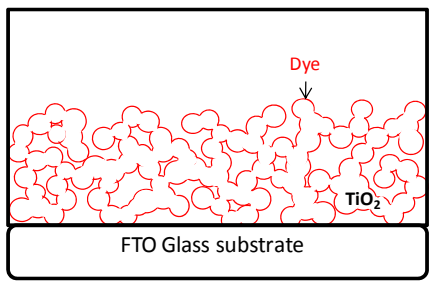

Figure B: Schematic of dyed $\mathrm{TiO}_{2}$ on FTO.

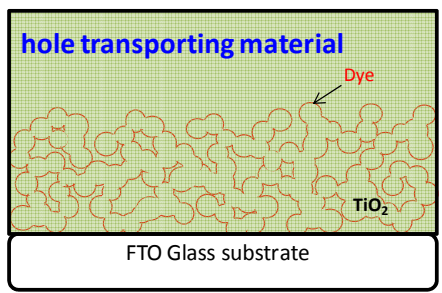

Figure D: Schematic of ideal photo-anode with the pores in the dyed $\mathrm{TiO}_{2}$ nanomatrix are completely filled by hole transporting material (PEDOT).

Figure 4.22: The schematic of photo anode presented (A) Bare $\mathrm{TiO}_{2}$ (B) Dyed $\mathrm{TiO}_{2}$ (C) Partial filling of PEDOT into dyed $\mathrm{TiO}_{2}$ and (D) Ideal photo-anode completely filled by PEDOT (hole transporting material).

\subsubsection{Calculation of total polymerization charge}

The total polymerization charge was calculated by integration of the polymerization currents. It was observed that the total polymerization charge was highest (14.8 $\mathrm{mC} / \mathrm{cm}^{2}$ ) when PEDOT was grown into $\mathrm{Z907}$ dyed $\mathrm{TiO}_{2}$ matrix for 30 minutes under constant anodic irradiation of $22 \mathrm{~mW} / \mathrm{cm}^{2}$ light intensity, and lowest $\left(1.6 \mathrm{mC} / \mathrm{cm}^{2}\right)$ for 2 minutes irradiation (at $22 \mathrm{~mW} / \mathrm{cm}^{2}$ ). The polymerization charge varied depending on the light intensity and period of PEDOT growth, increasing from 1.6 $\mathrm{mC} / \mathrm{cm}^{2}$ for 2 minute PEDOT deposition to $14.8 \mathrm{mC} / \mathrm{cm}^{2}$ for 30 minutes deposition. The total polymerization charge influenced the overall PV performance of various growth of PEDOT is shown in Table 4.2. More PEDOT in the photo-anode resulted 
increase of open-circuit voltage, short-circuit current density, fill factor and overall efficiency of the devices.

Table 4.2: Summary of total polymerisation charge and its effect on solid-state dyesensitized solar cells.

\begin{tabular}{|c|c|c|c|c|c|c|}
\hline $\begin{array}{c}\text { Periods of } \\
\text { PEDOT } \\
\text { growth } \\
(\mathrm{min})\end{array}$ & $\begin{array}{c}\text { Open } \\
\text { circuit } \\
\text { voltage } \\
\left(V_{\text {oc }}\right) \\
(\mathrm{mV})\end{array}$ & $\begin{array}{c}\text { Current } \\
\text { density } \\
\left(J_{\mathrm{sc}}\right) \\
\left(\mathrm{mA} / \mathrm{cm}^{2}\right)\end{array}$ & $\begin{array}{c}\text { Fill } \\
\text { factor } \\
(\mathrm{FF})\end{array}$ & $\begin{array}{c}\text { Efficiency } \\
(\eta) \%\end{array}$ & $\begin{array}{c}\text { Intensity of } \\
\text { light } \\
\left(\mathrm{mW} / \mathrm{cm}^{2}\right)\end{array}$ & $\begin{array}{c}\text { Total } \\
\text { charge } \\
\left(\mathrm{mC} / \mathrm{cm}^{2}\right)\end{array}$ \\
\hline 30 & 700 & 6.42 & 0.67 & 3.01 & 22 & 14.8 \\
\hline 15 & 625 & 4.74 & 0.67 & 1.98 & 22 & 12.2 \\
\hline 8 & 610 & 3.80 & 0.64 & 1.48 & 22 & 12.4 \\
\hline 4 & 345 & 0.21 & 0.35 & 0.03 & 22 & 4.08 \\
\hline 2 & 420 & 0.00 & - & 0.03 & 22 & 1.68 \\
\hline
\end{tabular}

\subsubsection{Current-voltage (JV) and incident photon to current conversion efficiency (IPCE) measurement}

The sandwich-type devices were fabricated by clipping the counter electrode (Goretex ${ }^{\circledR}$-Au-PEDOT) to the photo-anode. Figure 4.23 presents the JV curves for three solid-state dye-sensitized solar cells, measured here under 1 sun $\left(100 \mathrm{~mW} / \mathrm{cm}^{2}\right.$ light intensities, 1.5 AM Global). It was observed that when the PEDOT was grown for 30 minutes, higher $V_{\mathrm{oc}}, J_{\mathrm{sc}}$, FF and overall efficiency were obtained. The use of less time for PEDOT growth led to low device performance. All PV parameters for the solid-state dye-sensitized solar cells exhibited linear dependence on the amount of PEDOT deposited. Unfortunately, the photo-polymerization stopped after 30 
minutes and the photo-current declined to zero, which has already been discussed in Chapter 3 (section 3.3.2.2) previously.

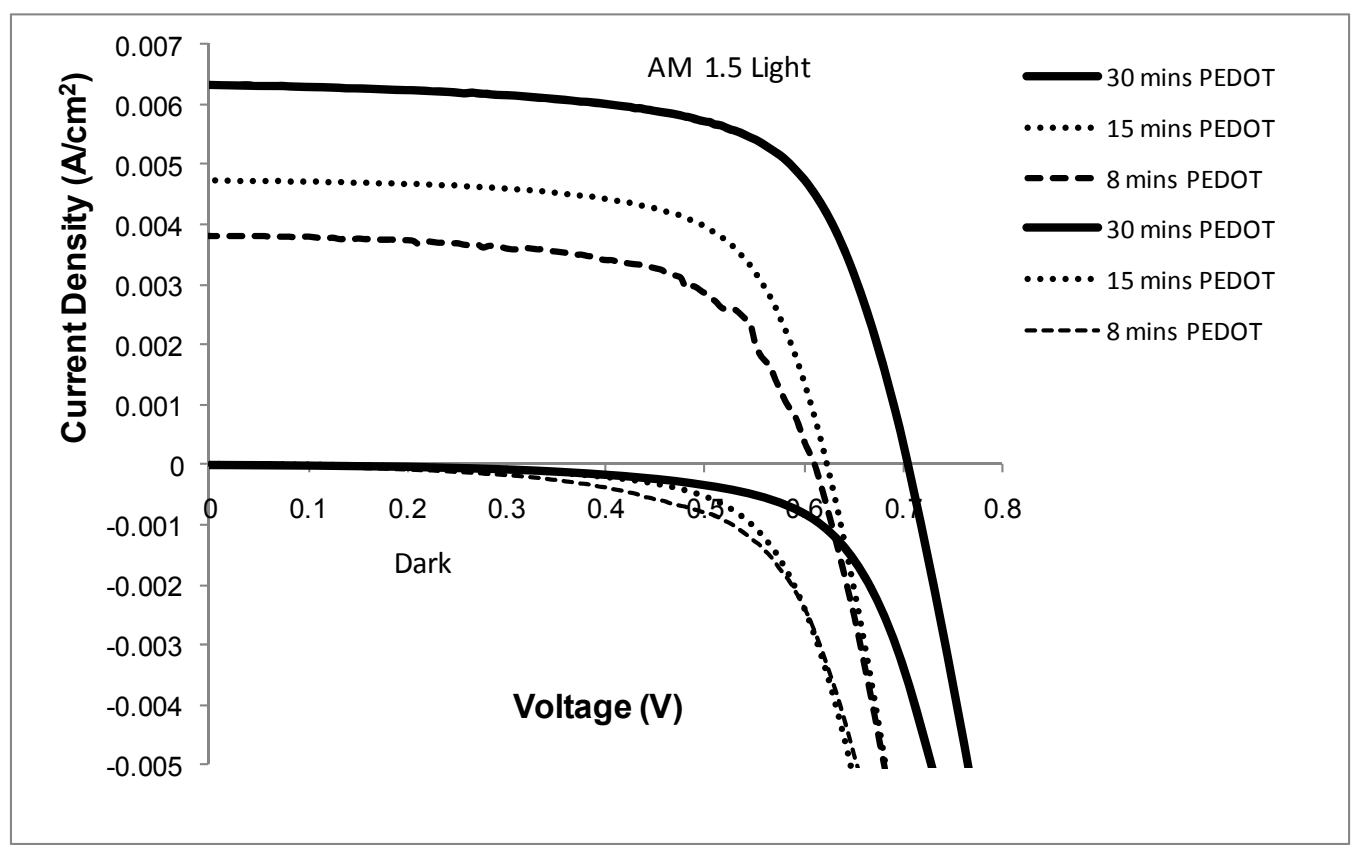

Figure 4.23: Photocurrent-voltage characteristics of PEDOT based ssDSCs fabricated by anodic side irradiation under AM 1.5, and their dark currents. The best performance of the device achieved when PEDOT was grown for 30 minutes.

The incident photon to current conversion efficiency (IPCE) was measured using our in-house custom set up for IPCE measurements. The height of the sensitization effect also decreased with a decrease in the amount of deposited PEDOT, as seen from Figure 4.24. The broad absorption peak at $518 \mathrm{~nm}$ is due to Z907 dye sensitization effect, and while beyond $600 \mathrm{~nm}$ a very long tail was due to the $\pi-\pi *$ transition of PEDOT. From Figure 4.24, it can be concluded that PEDOT does not absorb much in the UV-Vis region. The transparency of PEDOT in this region makes it a potential hole transporting material for solid-state dye-sensitized solar cells. 


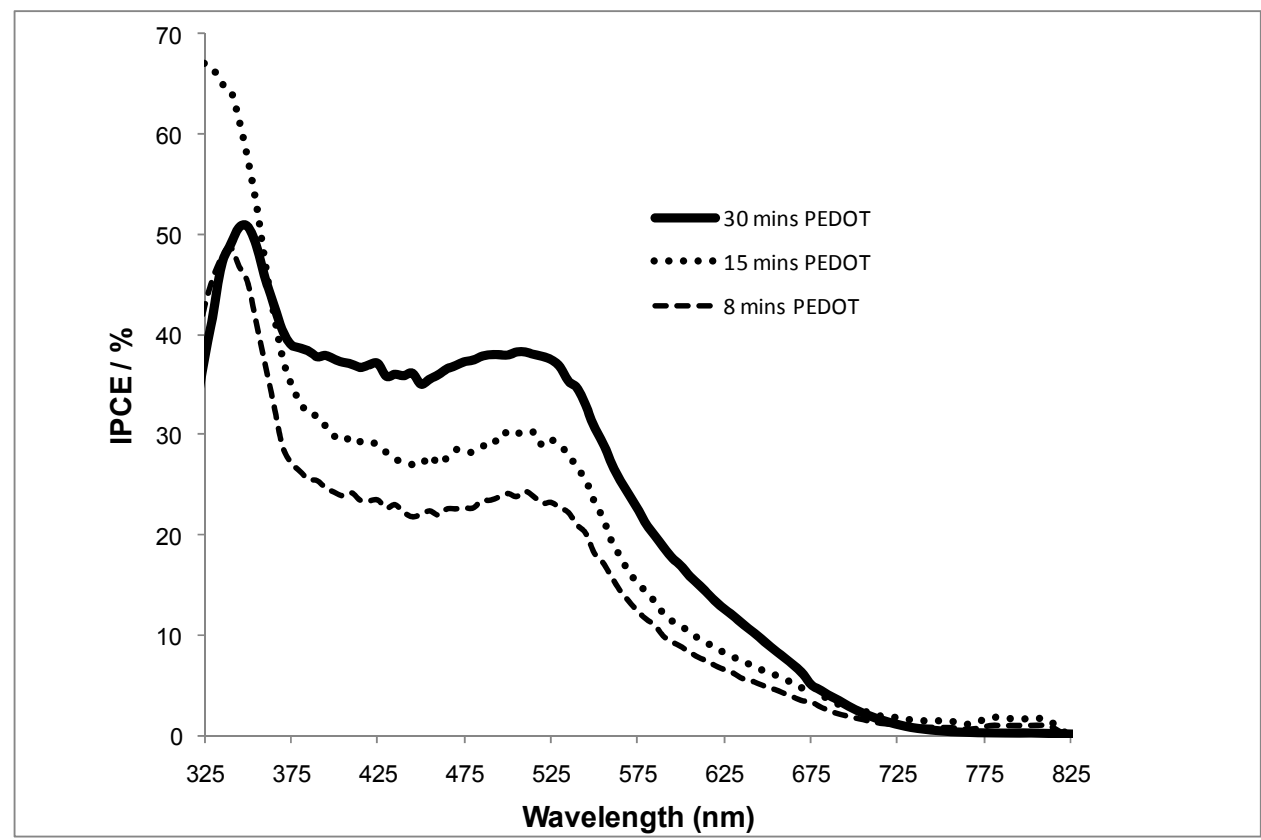

Figure 4.24: IPCE spectrum of PEDOT based ssDSCs fabricated by photoelectrochemical polymerization. The variation in IPCE with different quantities of deposited PEDOT is shown.

The photovoltaic parameters for the five PEDOT based solid-state dye-sensitized solar cells are presented in Figure 4.25. It is observed that $V_{\mathrm{oc}}$ increased markedly (approximately two-fold, from $345 \mathrm{mV}$ to $610 \mathrm{mV}$ ), when PEDOT growth time was increased from 4 minutes to 8 minutes. However, further increase in PEDOT growth time led to a slight increase in $V_{\text {oc }}$ (from $610 \mathrm{mV}$ to $700 \mathrm{mV}$ ). The same increasing trend was also observed for $J_{\mathrm{sc}}$ and $\mathrm{FF}$, resulting in the same increasing trend in overall photovoltaic efficiency. The PV parameter values for the five different ssDSCs are also presented earlier in Table 4.2. The data confirm that the amount of deposited PEDOT plays an important role in the overall performance of the devices.

The time limit of the PEDOT growth is already demonstrated in the mechanism in seection 3.2.2.5 (Chapter 3). This time growth is limited by materials what were used in the experiment. That's why we tried to use ionic liquid as media instead of acetonitrile but even if PEDOT were grown more than 30 mins but the efficiency of the cell dropped. From experimental findings we can conclude that it is not the 
amount of PEDOT grown into $\mathrm{TiO}_{2}$ but it depends on how uniformly it has grown in pores of dyed $\mathrm{TiO}_{2}$. The nature of the PEDOT growing can be confirmed through the trend of photo-polymerization current curve (section 3.2.2.5, Chapter 3 ). If the growth time could be extended beyond $30 \mathrm{~min}$, would more PEDOT be able to incorporate into the photo-anode and hence it would affect the cell performance because the slow dye regeneration kinetics can be minimized. Moreover, the low ratio of dye to PEDOT can be minimized. The enhanced efficiency could also be attributed to create the better dye and PEDOT interface.

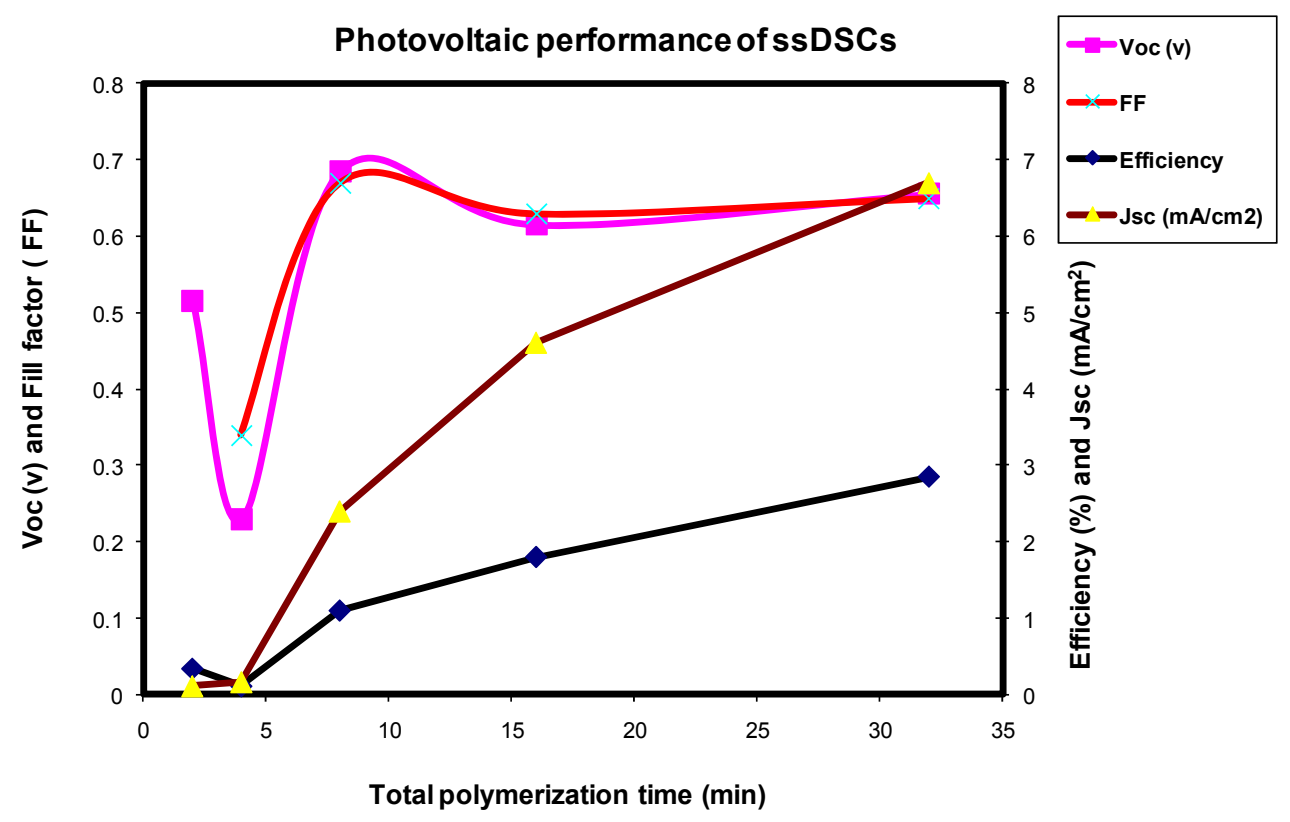

Figure 4.25: PV parameters for solid-state dye-sensitized solar cells where the PEDOT on the photo-anode was grown for various periods.

\subsubsection{Transient absorption spectroscopy (TAS) studies}

It was observed from transient absorption spectroscopy (TAS) studies that the dye regeneration kinetics occurred in a microsecond time frame for an efficient PEDOT based solid-state dye-sensitized solar cell. This is slower than in solar cells using the 
conventional $\mathrm{I}^{-} / \mathrm{I}_{3}^{-}$or spiro-OMeTAD for the redox couple. This slow kinetics is believed to be due to the low ratio of Z907 dye to PEDOT in the photo-anode. Photoanodes were prepared by our standard established methods [22]. We have grown the different amounts of PEDOT within the film by varying the PEDOT growth time. The total charge developed during photo polymerization decreased logarithmically when we decreased the PEDOT growth time. It is assumed that the ratio of ruthenium based dye (Z907) to bis-EDOT is approximately 1.2 when PEDOT was grown for a maximum of 30 minutes. The transient absorption signals of $\mathrm{TiO}_{2} / \mathrm{Z} 907$ dye films containing various amounts of PEDOT grown over different periods of time and grown at different probe wavelengths $(560 \mathrm{~nm}, 700 \mathrm{~nm}, 900 \mathrm{~nm})$ have been presented in our recent Journal of the American Chemical Society (JACS) paper [22]. All films were treated with ionic liquid (EMITFSI) before the TAS studies. The details of the TAS studies have been covered in our JACS paper [22]. The lower efficiency of the solid-state DSC is attributed to nano-porous nature of photo-anode substantially filled with PEDOT which lowered the recombination rate of dye cation and increased the dye regeneration rate.

\section{4 Conclusions}

This study has shown that PEDOT-based solid-state dye-sensitized solar cells with $3 \%$ efficiency can be fabricated. This efficiency can be reproduced if the various PEDOT growth conditions described above are maintained. The overall PV performance is found to be significantly influenced by the time of PEDOT growth, light intensity, dopant anions, and solvent during photo-electrochemical polymerization. It has been also observed earlier Chapter that PEDOT can not be grown beyond 30 minutes into a dyed $\mathrm{TiO}_{2}$ nanomatrix. PEDOT chains get longer with time and longest recorded is 17 unit of bis-EDOT. It was observed that there were still large numbers of pores in the nano-matrix dyed $\mathrm{TiO}_{2}$ unfilled by PEDOT (SEM and AFM studies). Moreover, photovoltaic performance of the ssDSCs depends on the amount of PEDOT grown into photo-anode. From UV-Vis-NIR and Raman spectra, it was observed that the conductivity of PEDOT was moderate. 
Therefore, to make more efficient ssDSCs, the PEP process needs further optimisation, by searching for alternative dyes, hole transporting material, or conditions that will fill all the pores of the HTM.

\section{5 References}

1. Ding, I.K., Tetreault, N., Brillet, J., Hardin, B.E., Smith, E.H., Rosenthal, S. J., Sauvage F., Grätzel, M., and McGehee, M.D., Advanced Functional Materials, 19 (2009) 1-6.

2. Yum, J.H., Chen, P., Grätzel, M., and Nazeeruddin, M.K., ChemSusChem, 1 (2008) 699-707.

3. Leventis, H.C., King, S.P., Sudlow, A., Hill, M.S., Molloy, K.C., and Haque, S.A., Nano Letters, 10 (2010) 1253-1258.

4. Armstrong, N.R., Veneman, P.A., Ratcliff, E., Placencia, D., and Brumbach, M., Accounts of Chemical Research, 42 (2009) 1748-1757.

5. Imahori, H., and Umeyama, T., Journal of Physical Chemistry C, 113 (2009) 9029-9039.

6. Potscavage, J., William, J., Sharma, A., and Kippelen, B., Accounts of Chemical Research, 42 (2009) 1758-1767.

7. Heremans, P., Cheyns, D., and Rand, B.P., Accounts of Chemical Research, 42 (2009) 111740-1747.

8. Dolan, A.R. and Wood, T.D., Journal of the American Society for Mass Spectrometry, 15 (2004) 893-899.

9. Mozer, A. J., Panda, D. K., Gambhir, S., Winther-Jensen, B., and Wallace, G.G., Journal of the American Chemical Society, 132 (2010) 9543-9545.

9a. Reynolds, J et.al Physical Review B, 67, (2003) 115205.

9b. Mozer, A. J., Panda, D. K et.al, Langmuir, 26 (2010) 1452-1455.

10. Nazeeruddin, M.K., Zakeeruddin, S.M., Humphry-Baker, R., Jirousek, M., Liska P., Vlachopoulos, N., Shklover, V., Fischer, C.H., and Grätzel, M., Inorganic Chemistry, 38 (1999) 6298-6305. 
11. Schmidt-Mende, L., Kroeze, J.E., Durrant, J.R., Nazeeruddin, M.K., and Grätzel, M., Nano Letters, 5 (2005) 1315-1320.

12. Reyes-Reyes, M., Cruz-Cruz, I., and Lopez-Sandoval, R., Journal of Physical Chemistry C, 114 (2010) 20220-20224

13. Chiu, W.W., Travas-Sejdic, J., Cooney, R.P. and Bowmaker, G.A., Journal of Raman Spectroscopy, 37 (2006) 1354-1361.

12. Choi, H.C, Mee, J.Y., and Kim, and S.Bin, Bulletin Korean Chemical Society, 25 (2004) 426.

13. Miao, L., Tanemura, S., Toh, S., Kaneko, K., and Tanemura, M., Journal of Crystal Growth, 264, (2004) 246-252.

14. Zhang, Y.H., Chan, C.K., Porter, J.F. and Guo, W., Journal of Material Research, 13 (1998) 2602.

15. Lei, Y., Zhang, L.D. and Fan, J.C., Chemical Physics Letters, 338 (2001) 231.

16. D. Bersani and P.P. Lottici, Applied Physics. Letters, 72 (1998) 73.

17. Likodimos, V., Stergiopoulos, T., Falaras, P., Harikisun, R., Desilvestro, J., and Tulloch, G., Journal of Physical Chemistry C, 113 (2009) 9412-9422.

18. Selvaganesh, S.V., Mathiyarasu, J., Phani, K.L.N, and V. Yegnaraman., Nanoscale Research Letters, 2 (2007) 546-549.

19. Garreau, S., Louarn, G., Buisson, J. P., Froyer G., and Lefrant, S., Macromolecules, 32 (1999) 6807-6812.

20. Mozer, A. J., Panda, D. K., Gambhir, S., Romeo, T., Winther-Jensen, B., and Wallace, G.G., Langmuir, 26 (2010) 1452-1455. 


\section{NOVEL PEDOT BASED COUNTER ELECTRODES FOR SOLID-STATE DYE- SENSITIZED SOLAR CELLS}




\section{1 Introduction}

Dye-sensitized solar cells (DSCs) have been demonstrated to be a viable, low cost method of producing electricity through the use of a mesoporous inorganic semiconductor sensitized with various dyes and $\mathrm{Au}, \mathrm{Ag}$ or Pt metalized counterelectrodes [1, 2]. Considerable research has been carried out on the working electrode. However, although the counter-electrode also plays a key role by catalyzing the reduction of the oxidised part of the redox mediator (see Chapter 1), it has attracted much less study to date. A high-performance DSC requires the counterelectrode to be highly catalytic and conductive. Therefore, platinum (Pt) has been the most prolific metalized counter-electrode used. However, while Pt is a very good catalyst, it is very expensive and increases the price of the DSCs. Similar issues have arisen from the use of $\mathrm{Au}$ or $\mathrm{Ag}$ metalized counter-electrodes. Thus, other materials have been investigated to replace these expensive counter-electrode materials such as carbon black, carbon nanotubes, polymers and composites of polymer/Pt or polymer/carbon [3-8]. However, only a few studies have been done on counter electrodes for solid-state dye-sensitized solar cells (ssDSCs).

\subsubsection{The importance and role of the counter electrode in ssDSCs}

The counter electrode should selectively extract positive charge from the photoanode ensuring a one directional flow of electrons. It should be compatible with the photo-anode (by providing more physical contact between photo-anode and counter electrodes). The excellent electron transfer catalysis properties of the counter electrode make the device efficient. Electrons in the conduction band of semiconductor $\mathrm{TiO}_{2}$ and the holes of the hole transporting materials are collected by transparent conducting oxide, such as FTO, in the working electrode and the counter electrode respectively, to complete the electronic circuit. The higher the light reflectivity of the counter electrode, the better is the light harvesting, which will increase the incident photon to current efficiency (IPCE) resulting in an increase in overall photovoltaic performance [9]. 


\subsubsection{The ideal properties of counter electrode for ssDSCs}

The most important and ideal properties of the counter electrodes are:

(i) It must be highly conductive.

(ii) It should be chemically/ electrochemically and thermally stable.

(iii) Its work function must match that of the oxidised hole conductor Fermi level of the hole conductor (oxidation potential of the $\operatorname{PEDOT}^{+}$). This ensures no electronic barrier at the interface between dyed $\mathrm{TiO}_{2}$ and hole conductors.

(iv) For applications including see-through windows, it should be transparent.

(v) A thinner counter electrode is more transparent and will enable more lightharvesting.

(vi) It should preferably be flexible and compressible for wearable solar cell applications.

(vii) It should be light weight, and made of cheap materials that are readily available and easily processed by the electronics industry.

(viii) It should be easy to produce.

\subsubsection{Counter electrodes that have been used to date for ssDSCs}

Prior to this study, gold represent the most common counter electrode material for solid-state dye-sensitized solar cells using a photo-electrochemically deposited polyethylenedioxythiophene (PEDOT) hole conductor [5]. A gold counter electrode formed by metal vapour deposition has also been utilised for spiro-OMeTAD based solid-state dye-sensitized solar cells [10]. Snaith et al. have also reported [10a] replacing the dissipative gold counter electrode with a reflective $\mathrm{Ag}$ (work function = $4.4 \mathrm{eV}$ ) counter electrode, which enhanced the light absorption of the device. A silver based counter electrode increased the overall power conversion efficiency to $5.1 \%$ for the spiro-OMeTAD based solid-state DSC [10a]. The high work function $(5.1 \mathrm{eV})$ of gold also makes it a good choice as a counter electrode for organic photovoltaics. 
Recently Yanagida et al. [10b] used a high work function material, vanadium pentoxide $\mathrm{V}_{2} \mathrm{O}_{5}(4.7 \mathrm{eV})$ with aluminium $(4.3 \mathrm{eV})$, as a composite electrode for spiro-OMeTAD based solid state DSC which can replace the Ag and the power conversion efficiency goes up to $2 \%$. Moreover, this $\mathrm{V}_{2} \mathrm{O}_{5} / \mathrm{Al}$ composite novel counter electrode costs one tenth or even less than the Ag one [10b]. But, when only pure $\mathrm{Al}$ was used as a counter electrode, it has been reported [10b] that most devices are short circuited because the work functions of FTO $(4.3 \mathrm{eV})$ and $\mathrm{Al}(4.3 \mathrm{eV})$ are the same. The internal electrical field developed under illumination is the key driving force for the movement of electrons and holes towards the cathode and anode respectively in case of polymer solar cells. But this internal electrical field is determined by the difference between the work function values of two electrodes (working electrode and counter electrode) when the active layer thickness remains same. Solid-state DSC is very similar to polymer thin solar cells (e.g. hole-electron separation at the interface of fullerene derivative $[6,6]$-phenyl- $\mathrm{C}_{61}$-butyric acid methyl ester (PCBM) and Poly (3-Hexylthiophene (P3HT) [10b]. However, in solidstate DSCs, dyed $\mathrm{TiO}_{2}$ acts as a light harvester and in the same time as an electron transporter and the charge separation takes place in between dyed $\mathrm{TiO}_{2}$ and hole transporting materials.

Similarly, for liquid electrolyte dye-sensitized solar cells, the counter electrode facilitates the reduction of $\mathrm{I}_{3}{ }^{-}$ions generated after electron injection from iodide into the oxidized photo-sensitizers. FTO (work function $=4.3 \mathrm{eV}) /$ ITO $($ work function $=$ $4.7 \mathrm{eV}$ ) transparent conducting glass substrates show slow electron transfer kinetics for tri-iodide $\left(\mathrm{I}_{3}^{-}\right)$reduction. This is the reason why a thin layer of metallic Pt/Au is sputter coated onto the FTO/ITO substrate in order to catalyze the cathodic reduction of $\mathrm{I}_{3}{ }^{-}$ions and redox couple regeneration. However, unsurprisingly the gold coating is very expensive. To date, limited studies have been made on the role of different counter electrodes (such as $\mathrm{Pt} / \mathrm{Au}$ ) for PEDOT based solid-state dye-sensitized solar cells.

Therefore, there is an urgent need for the development of a counter electrode that is conductive, flexible, and compressible to solve the above mentioned problems. Furthermore, commercial mass production of solid-state dye-sensitized solar cells 
will still require large quantities of gold which is not abundantly available [6]. Therefore, it is desirable to develop cost-effective alternative materials that are readily available, and provide comparable photovoltaic performance in solid-state dye-sensitized solar cells.

\subsubsection{Introduction to counter electrodes developed in this chapter}

We have investigated three new materials that address the above criteria for counter electrodes for solid-state dye-sensitised solar cells, namely:

1. Conjugated polymer-based counter electrodes.

2. Graphene-based counter electrodes.

3. A novel compressible Goretex ${ }^{\circledR}-\mathrm{Au}-\mathrm{PEDOT}$ based counter electrode.

\subsubsection{Conjugated polymer-based counter electrodes}

Conducting polymers are promising candidates as alternative counter-electrode materials for DSCs due to their unique properties, such as low cost, good conductivity, remarkable stabilities, good specific capacitances, and catalytic activity for $\mathrm{I}_{3}{ }^{-}$reduction [11-15]. However, there are only a few reports on the use of conducting polymers as counter-electrode materials in DSCs [15-18]. Polypyrrole (PPy) is one of the most intensively studied conducting polymers over the last decade, although it is less prevalent within the context of DSCs [11-17]. PEDOT is one of the most robust conducting polymers with several attractive properties, including its low redox potential, high stability, good electrical conductivity and optical transparency in the doped state $[18,19]$. In addition, and more importantly for DSCs, it shows high catalytic activity for $\mathrm{I}_{3}^{-}$reduction and has therefore received much attention as a potential counter electrode material [20-23, 32]. The fabrication cost of these conducting polymer counter-electrodes is lower than that of Pt or $\mathrm{Au}$ electrodes [7]. Electrochemical process is an easy method to make large quantities of conducting polymer-based counter electrodes to replace precious metal-based 
electrodes such as gold and platinum. Recently, such conducting polymer counter electrodes have been used for dye-sensitized solar cells [32].

\subsubsection{Graphene-based counter electrodes}

Carbon has four allotropic forms: graphene, diamond, fullerene and graphite. Out of these, graphene has been investigated much less for photovoltaic applications. Graphene, as a carbon based electrode for solid-state dye-sensitized solar cells, has generated a great deal of recent interest [24] because it is cheap and readily available from graphite which is abundant in nature. It may be used as a counter electrode to replace FTO/ITO glass substrates which are very expensive and susceptible to instability towards acidic, alkali and high temperature environments [24].

Graphene is a single layer structure of graphite possessing properties of good chemical and thermal stability, high transparency (both in visible and near infra-red regions), high strength, excellent conductivity, ultra-smooth surface with good wettability; making it a good potential counter electrode for optoelectronics devices $[25,26]$. Recently ultrathin graphene films with a conductivity of $550 \mathrm{~S} / \mathrm{cm}$ and transparency of more than $70 \%$ over the 1000-3000 nm wavelength range have been used as working electrodes by Müllen et al. for spiro-OMeTAD based solidstate dye-sensitized solar cell with an overall power conversion efficiency of approximately $0.3 \%$ [24].

\subsubsection{Goretex ${ }^{\circledR}-A u-P E D O T$ counter electrode}

Goretex ${ }^{\circledR}$ is a synthetic engineered fabric membrane made of PTFE (polytetrafluorethylene) having a triple layer which provides an impenetrable barrier towards air and $\mathrm{H}_{2} \mathrm{O}$, without affecting its breathability properties. It is extensively used as a fabric for outdoor clothes. Goretex ${ }^{\circledR}$ membrane has microscopic pores which restrict the passage of larger water droplets while permitting the passage of smaller water vapour molecules. Due to the semi-permeable qualities of the 
Goretex ${ }^{\circledR}$ membrane, extensive research has been carried out on various applications such as for catalysis, energy conversion and energy storage [27, 28].

Composite Goretex ${ }^{\circledR}-\mathrm{Au}-\mathrm{PEDOT}$ electrodes were developed and supplied to us by our collaborator, Dr. Bjorn Winther-Jensen at the ARC Centre of Excellence for Electromaterials Science, Monash University. This counter electrode has been proven as a potential counter electrode for $\mathrm{O}_{2}$ reduction catalyst for two energy applications in fuel cells and metal-air batteries [27, 28]. It was found that the reduction rates of $\mathrm{O}_{2}$ were comparable with conventional and expensive metal platinum catalysts for energy storage and energy generation applications. This Goretex ${ }^{\circledR}-\mathrm{Au}-\mathrm{PEDOT}$ electrode is highly stable over several hours of operation.

Porous materials such as the flexible and compressible Goretex ${ }^{\circledR}$ membrane coated with PEDOT (via vapour phase polymerization) may meet the criteria required for good counter electrodes in solid-state DSCs, and have therefore also been examined in this chapter. Goretex ${ }^{\circledR}-\mathrm{Au}-\mathrm{PEDOT}$ has low sheet resistance. This compressible, flexible, highly conductive current-collecting electrode based on vapor-phase polymerized PEDOT on a gold-Goretex ${ }^{\circledR}$ membrane having $60 \%$ compressibility and $80 \%$ porosity make this novel electrode tolerant of defects in the $\mathrm{TiO}_{2}$ photo anode. Its compressibility, flexibility and surface roughness have proven to be an ideal matching current collector counter electrode for the dyed $\mathrm{TiO}_{2}$ (working electrode) surface; this will be discussed in detail.

\section{2 Methods and preparation}

The photo-anodes were prepared using our standard photo-electrochemical deposition technique, described in Chapter 3. Therefore, we will discuss only the various methods of preparation of the counter electrodes in sections 5.2.1-5.2.4 below. 


\subsubsection{Sputtered Au on ITO electrodes}

Sputter coating is a physical vapour deposition technique where a thin layer of the target metal (Pt, $\mathrm{Au}, \mathrm{Pd}, \mathrm{Ni}, \mathrm{Ti}$, etc) is etched and deposited on the desired substrate. In the present study, magnetron sputtering was used for deposition of $\mathrm{Au}$ or $\mathrm{Pt}$ on ITO counter electrodes. Firstly, ITO coated glass was cleaned with ethanol followed by UV-ozone. The ITO was then sputter-coated with a thin layer of gold/ platinum using a Dynavac Magnetron Sputter Coater Model SC100MS. The sputtering conditions employed were an Ar pressure of $2 \times 10^{-3}$ mbar and a sputtering current of $50 \mathrm{~mA}$. The deposition rate of the Pt or gold was $2 \AA / \mathrm{s}$, and thicknesses of $10-30 \AA$ were obtained depending on the sputtering period.

\subsubsection{Conjugated polymer-based counter electrodes}

The vapour-phase polymerization (VPP) process for depositing PEDOT on the FTO glass counter electrode was performed according to literature method [29-31] using the EDOT monomer (Baytron M) and Fe (III) tosylate (40\% solution in butanol containing 0.5 M pyridine), both received from Bayer AG. After the polymerization, the sample was washed twice in ethanol to remove excess Fe and Fe-tosylate and EDOT.

Polypyrrole (PPy) counter electrodes were prepared by spin-coating the oxidant (Fe (III) p-toluene sulfonate in 60\% 1-butanol containing 5\% PEG-PPG, MWt 12,000) onto the FTO substrate at $1000 \mathrm{rpm}$ for $15 \mathrm{sec}$ and then followed by VPP of PPy as previously described [29-31].

For comparison purposes, PEDOT counter-electrodes were also prepared by chronoamperometry via the polymerization of bis-EDOT at a constant potential of $0.8 \mathrm{~V}$ vs. $\mathrm{Ag} / \mathrm{AgCl}$, commensurate with the oxidation potential of the bis-EDOT. The polymerization was carried out at room temperature for 3 minutes. Using this method, PEDOT polymer films could be grown on particular conductive substrates to desired thickness. Moreover, in this method no catalyst or chemical oxidant was 
used, so high purity of the film could be achieved. Characterisation of the films produced was carried out using the spectroscopic techniques of UV-Vis-NIR, Raman, and FT-IR.

\subsubsection{Graphene-based counter electrodes}

Chemically converted graphene (CCG) papers were made using the procedure reported by Li et al. [33]. This involved filtration of a $0.5 \mathrm{mg} / \mathrm{ml}$ aqueous dispersion of exfoliated CCG obtained by hydrazine reduction of a graphite oxide dispersion. Functionalised CCG was prepared by chlorination of the CCG paper by a novel method [34], resulting in further elimination of defects from the CCG paper. The thickness and therefore the conductivity are controlled by the number of layers of graphene. In this study, 6-10 $\mu \mathrm{m}$ films composed of graphene layers were used. The conductivity of thionyl chloride treated CCG (CCG-Cl) was found to be $4259 \mathrm{~S} / \mathrm{m}$, two times that of the CCG film $(2274 \mathrm{~S} / \mathrm{m})$. The thickness of these films was measured by surface profilometry (Veeco Dektak 150) and their conductivity determined by the four-point probe method (Jandel RM3). The likely structure of $\mathrm{CCG}$ is shown in Figure 5.1. The graphene layer was attached to the FTO glass by putting a few drops of the ionic liquid EMITFSI either directly onto conducting, transparent FTO layer on a supporting glass or onto the same substrate previously coated with PEDOT.

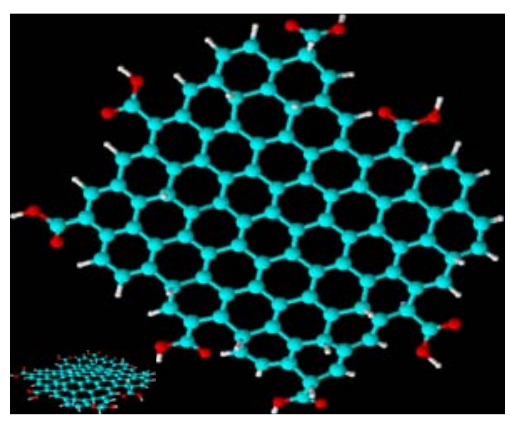

Figure 5.1: The structure of carboxylic acid functionalised graphene. 


\subsubsection{Goretex ${ }^{\circledR-A u-P E D O T ~ c o u n t e r ~ e l e c t r o d e s ~}$}

These electrodes were prepared and supplied by Dr Bjorn Winther-Jenssen according to a published procedure $[26,28]$. Briefly, a PTFE (Teflon $\left.{ }^{\circledR}\right)$ membrane (Goretex (®) was sputtered with a thin layer of gold. Then, one side of the porous Goretex ${ }^{\circledR}$ membrane was coated further with PEDOT by vapour phase polymerization method using iron tosylate as oxidant and EDOT as monomer. Scanning electron microscopy performed by Winther-Jensen et al. showed that the thickness of PEDOT was

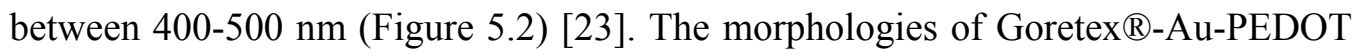
counter electrodes were examined by SEM. Figure 5.2 shows a typical SEM image, revealing very rough surface. The amount of PEDOT calculated by Winther-Jensen et al. for a $400 \mathrm{~nm}$ thickness of PEDOT was $0.05 \mathrm{mg} / \mathrm{cm}^{2}$ and the sheet resistance of this conducting polymer was $6 \Omega / \square$.

It has been observed by Winther-Jensen et al. [22] that this Goretex ${ }^{\circledR}-A u-P E D O T$ counter electrode is non-permeable to electrolytes but permeable to gases, which is highly suited to the three phase interface in oxygen reduction [27]. The PEDOT was well bonded to the Goretex ${ }^{\circledR}$ membrane; making this counter electrode stable, durable and flexible. The membrane was also wettable by various non-aqueous solvents such as ethanol [27]. The compressibility of a $80 \mu \mathrm{m}$ thick Goretex ${ }^{\circledR}-\mathrm{Au}-$ PEDOT electrode was found to be $60 \%$, which is close to the pristine Goretex $\mathbb{R}$ membrane compressibility value (55\%) given by the supplier. A very small force of only $10 \mathrm{mN} / \mathrm{mm}^{2}$ is needed to compress a $10 \mu \mathrm{m}$ deformation in this counter electrode. 


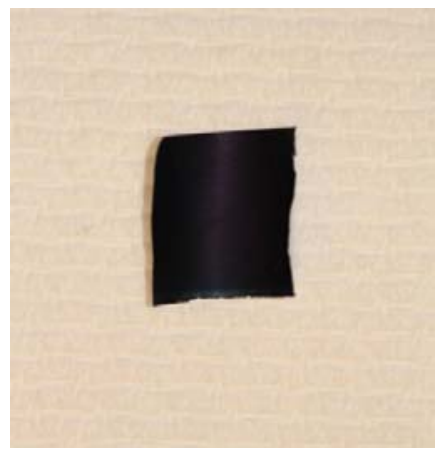

(A)

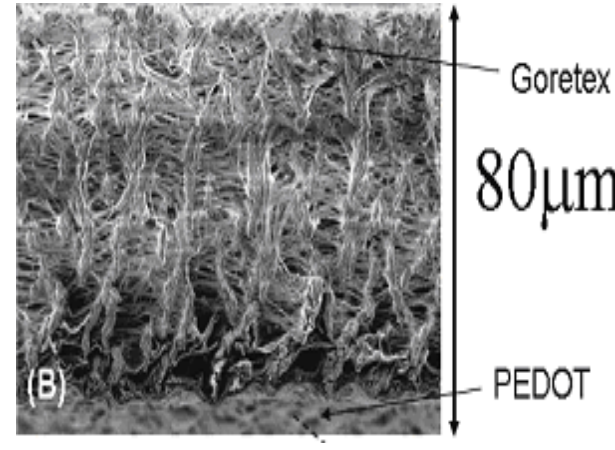

(B)

Figure 5.2: (a) Goretex ${ }^{\circledR}-A u-P E D O T$ used as counter electrode (Left) for solid-state DSCs and (B) Cross-sectional SEM image of a Goretex ${ }^{\circledR}-A u-P E D O T$ counter electrode (Right).

\section{3 Results and discussion}

\subsubsection{Sputtered Au/ITO electrode}

The main problem encountered with gold/ITO counter electrodes in our laboratory is their lack of reproducibility. This occurs when the photo-anode is uneven and not defect-free over a large area (Figure 5.3). Therefore, it is very difficult to clip with the same mechanical force when two rigid, flat and incompressible electrodes (photo-anode and $\mathrm{Au}$ ) are assembled in devices. It is known from experience that, in each solid-state DSC, the current-voltage (JV) graph is highly dependent on the pressure applied on two electrodes (working and counter) [23].

Figure 5.3 presents the current-voltage (JV) curves for two solid-state DSCs using gold counter electrode (30 $\mathrm{nm}$ thickness of Au on ITO substrate). The photovoltaic result should be reproducible, but when the photo-anodes employed varied in their surface morphology or when one was not defect-free, this did not occur. For example, Figure 5.3a shows the surface profiles of a photo-anode where two spike defects of about $1 \mu \mathrm{m}$ height are observed. These cause contact problems between the counter electrode and working electrode. The defects are probably due to the agglomeration of $\mathrm{TiO}_{2}$ nanoparticles or contamination by dust in air or any foreign material. It amply indicates that the electrical contact point between the two 
electrodes is low. This is attributed to the rigid, flat, non-flexible and incompressible nature of this gold-based counter electrode.

When photo-anode (having defects) as in Figure 5.3 and used with a rigid, flat $\mathrm{Au} / \mathrm{ITO}$ counter electrode (Figure 5.4) produced only $0.80 \%$ efficiency (Figure 5.3), with low current density $\left(3.5 \mathrm{~mA} / \mathrm{cm}^{2}\right)$ and low fill factor $(0.38)$. The higher efficiency device in Figure 5.3 has an efficiency of $2.9 \%$ which is the highest so far obtained with this fabrication procedure in the published literature. This can be attributed to its surface roughness, giving a higher number of physical contact points between photo-anode and counter electrode. Although solid-state DSCs that have been fabricated have with up to $2.9 \%$ efficiency with a Au/ITO counter electrode, giving a more efficient conversion rate, it was found that this efficiency would only be reproduced when the photo-anode was defect free. In practice in our laboratory, a low degree of reproducibility was generally found due to defects in the photo-anodes.

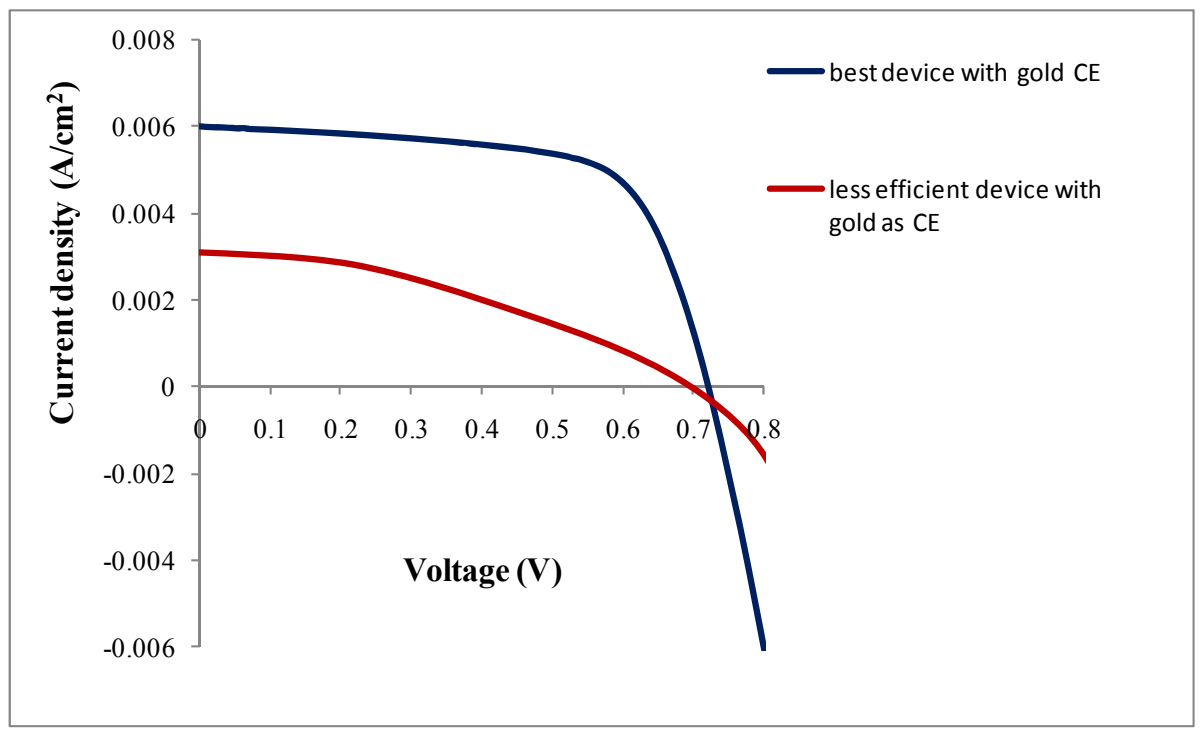

Figure 5.3: Current-voltage characteristics of two different solid-state DSCs under $100 \mathrm{~mW} \mathrm{~cm}^{-2}$ light intensities (AM 1.5 global) using Au/ITO as counter electrode. The difference in efficiency is attributed to differences in the surface roughness of the two photo-anodes. 


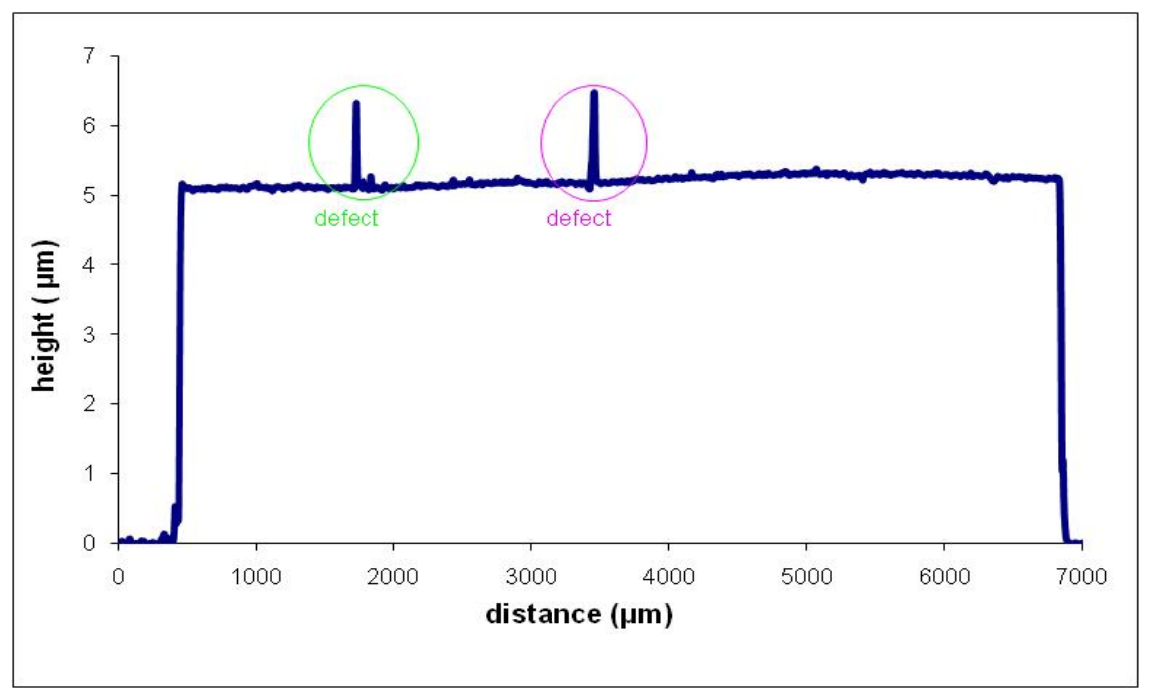

Figure 5.3a: Surface profile of a PEDOT- $\mathrm{Z} 907$ dyed $\mathrm{TiO}_{2}$ photo-anode showing the thickness as well as any defects on its surface.

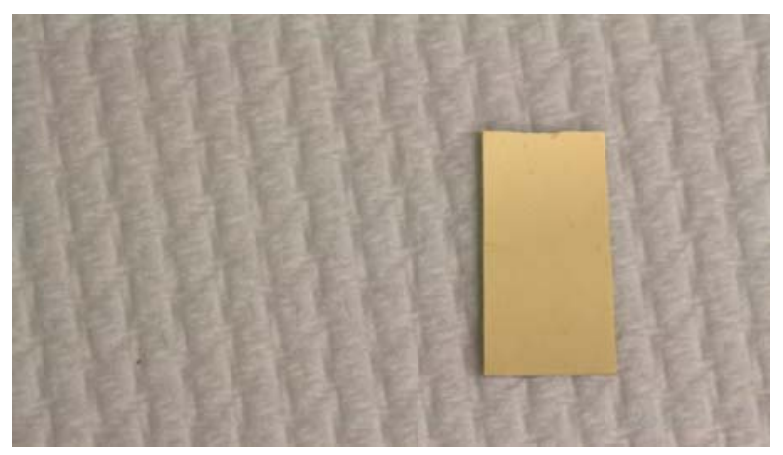

Figure 5.4: Gold sputtered on ITO substrate used as counter electrode.

\subsubsection{Conjugated polymer-based electrode}

\subsubsection{Growth of PEDOT by chronoamperometry}

The thickness of the PEDOT film can be controlled by varying the electropolymerization period. The chronoamperograms obtained during polymer growth are shown in Figure 5.5. 


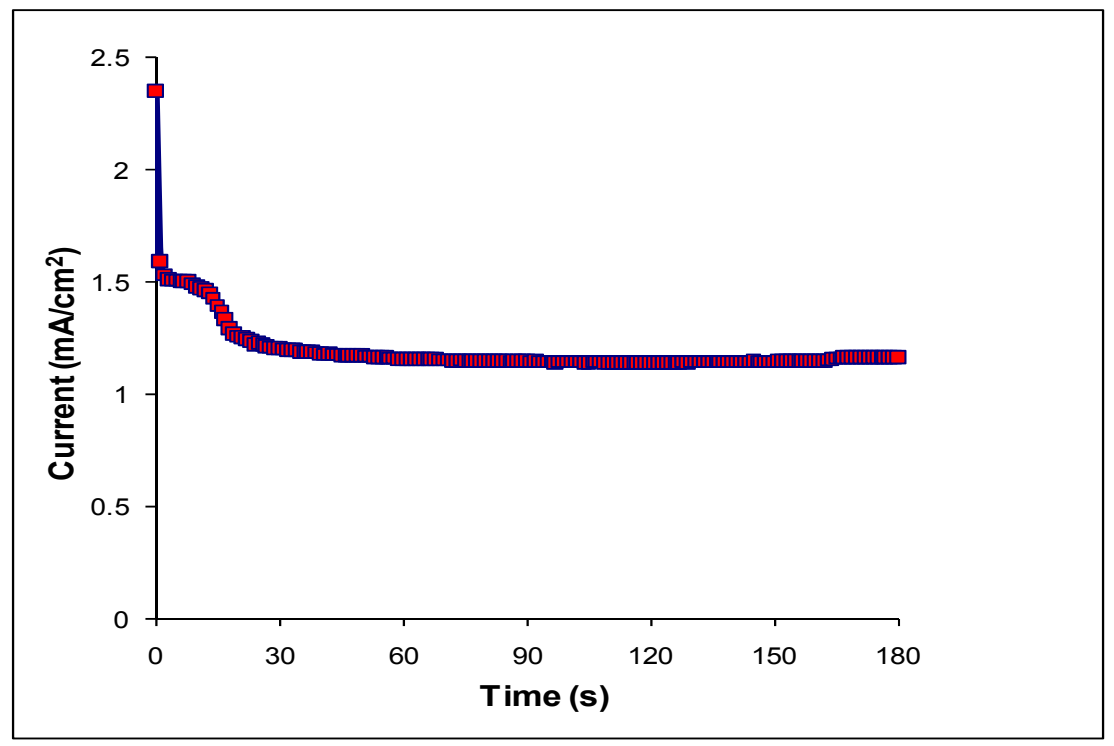

Figure 5.5: Chronoamperograms obtained during growth of PEDOT on FTO glass when applying a constant potential of $0.8 \mathrm{~V}$ (vs. $\mathrm{Ag} / \mathrm{AgCl}$ ) for $180 \mathrm{~s}$ using an acetonitrile solution of $0.01 \mathrm{M}$ bis-EDOT as electrolyte and $0.1 \mathrm{M}$ $\mathrm{LiClO}_{4}$ as dopant source $(\mathrm{Ag} / \mathrm{AgCl}$ as reference electrode and $\mathrm{Pt}$ mesh as counter electrode).

From Figure 5.5, it can be observed that the initial charging current increased up to $\sim 2.5 \mathrm{~mA} / \mathrm{cm}^{2}$ then decreased to a constant value of $\sim 1.2 \mathrm{~mA} / \mathrm{cm}^{2}$. The constant current over extended time is indicative of continued deposition on a conducting material (on FTO glass having resistance $\sim 10 \Omega / \square$, transmission $\sim 75 \%$ ) on to a constant surface area.

\subsubsection{Growth of PEDOT and Polypyrrole by vapour phase polymerization (VPP) method}

VPP of the PEDOT counter electrodes was performed following literature method [29-31]. The PEDOT composite films obtained had thicknesses of 0.5 to $1 \mu \mathrm{m}$ and typical conductivity of $>1000 \mathrm{~S} \mathrm{~cm}^{-1}$. 
The polypyrrole (PPy) counter electrodes were prepared by spin-coating the oxidant onto the FTO substrate and subsequent exposure to pyrrole vapour. The PPy film obtained had a thickness of $150 \mathrm{~nm}$ and conductivity of $\sim 20 \mathrm{~S} \mathrm{~cm}^{-1}$.

The current-voltage performance of solid-state DSCs using PEDOT, PPy and FTO counter electrodes were evaluated both in the dark and under one sun $\left(100 \mathrm{~mW} \mathrm{~cm}^{-2}\right)$ illumination (Figure 5.6). The solid-state DSCs with PEDOT and PPy counterelectrodes showed $2.66 \%$ and $2.50 \%$ of overall light to electric energy conversion efficiency, respectively. This is comparable to Pt or Au counter-electrode solid-state DSCs, that recorded a conversion efficiency of $2.8 \%$ and $2.91 \%$, respectively (Table 5.1). The bare FTO counter electrode solid-state DSCs gave a higher $\mathrm{V}_{\text {oc }}$ compared to the other counter electrodes, however, the fill factor was low, thereby reducing its overall efficiency. The low fill factor is due to the lower number of physical contact points between the working and counter electrodes (maybe due to difference in their surface roughness).

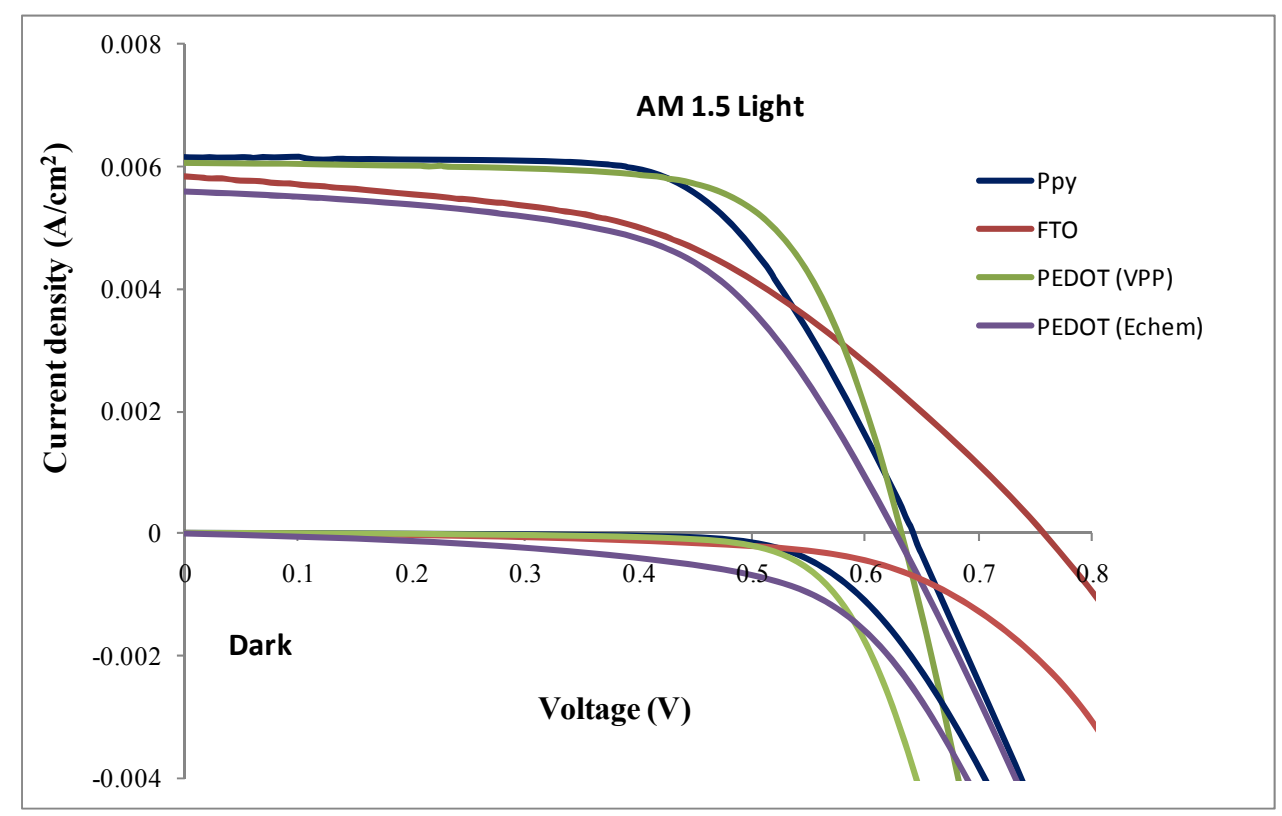

Figure 5.6: Current-voltage characteristics under $100 \mathrm{~mW} \mathrm{~cm}^{-2}$ light intensities (AM 1.5 global) and dark conditions for solid-state DSCs with different counter electrodes. Blue line for PPy, red line for FTO, olive green for VPP PEDOT and purple for electrochemically grown PEDOT. 
Although polypyrrole (PPy) is less conductive $\left(\sim 20 \mathrm{~S} \mathrm{~cm}^{-1}\right)$ than PEDOT $(\sim 1000 \mathrm{~S}$ $\left.\mathrm{cm}^{-1}\right)$, the performance of the PPy based solid-state DSC was comparable to that using PEDOT as counter electrode (Table 5.1). Vapour phase polymerized PEDOT is seen to give higher efficiency than a counter electrode using PEDOT grown by chronoamperometry. This is attributed to the higher conductivity and surface roughness of the vapour phase generated polymer film.

Table 5.1: Comparison of PV performances of solid-state DSCs with different counter electrodes.

\begin{tabular}{|c|c|c|c|c|c|}
\hline Counter Electrodes & $\begin{array}{c}V_{\mathrm{oc}} \\
(\mathrm{mV})\end{array}$ & $\begin{array}{c}J_{\mathrm{sc}} \\
\left(\mathrm{mA} \mathrm{cm}^{-2}\right)\end{array}$ & FF & $\begin{array}{c}\eta \\
(\%)\end{array}$ \\
\hline Au/ITO & 740 & 5.96 & 0.66 & 2.91 \\
\hline Pt/ITO & 670 & 7.57 & 0.55 & 2.80 \\
\hline PEDOT/ FTO (via VPP) & 635 & 6.06 & 0.69 & 2.66 \\
\hline Polypyrrole/ FTO (via VPP) & 645 & 6.16 & 0.63 & 2.50 \\
\hline PEDOT/ FTO ( via Chronoamperometry) & 703 & 5.85 & 0.56 & 2.22 \\
\hline
\end{tabular}

Note: $V_{\text {oc }}$ open-circuit voltage; $J_{\text {sc }}$ : short-circuit current density; FF: fill factor; $\eta$ : energy conversion efficiency.

\subsubsection{Graphene-based electrode}

The surface of the graphene layer was treated with a few drops of the ionic liquid EMITFSI prior to using as a counter electrode. This enhanced the electrical contact between working electrode (photo anode) and graphene based counter electrode. The current-voltage performance of $0.25 \mathrm{~cm}^{2}$ solid-state DSCs using both chemically converted graphene (CCG), thionyl chloride treated graphene (GGC-Cl) counter electrodes and PEDOT-coated FTO counter electrodes were evaluated both in the 
dark and under one sun illumination $\left(100 \mathrm{mWcm}^{-2}\right)$ and air mass $1.5 \mathrm{G}$ under identical conditions (Figure 5.7). The solid-state DSCs with CCG-Cl counterelectrode showed $2.22 \%$ of overall light to power conversion efficiency, which is an improvement on the PEDOT only solid-state DSC, that recorded a conversion efficiency of $2.12 \%$, and comparable to data obtained using the Au/ITO counterelectrode (Table 5.2). Although the Au/ITO counter-electrode gave a more efficient conversion rate, reproducibility was low. This low reproducibility is observed when the surface roughness of photo-anode is not well matched with the surface roughness of the Au-ITO counter electrode.

Table 5.2: Comparison of photovoltaic parameters obtained with different counter electrode materials in solid-state DSCs under one sun conditions (AM 1.5, $\mathrm{P}_{\text {in }} 100$ $\left.\mathrm{mW} \mathrm{cm}^{-2}\right)$.

\begin{tabular}{|c|c|c|c|c|}
\hline $\begin{array}{l}\text { Counter } \\
\text { Electrode }\end{array}$ & $\begin{array}{l}\mathrm{V}_{\mathrm{OC}} \\
(\mathrm{mV}) \\
( \pm 3 \% \text { of } \\
\text { mean } \\
\text { value })\end{array}$ & $\begin{array}{l}\mathrm{J}_{\mathrm{SC}} \\
\left(\mathrm{mA} / \mathrm{cm}^{2}\right) \\
( \pm \quad 15 \% \\
\text { of mean } \\
\text { value })\end{array}$ & $\begin{array}{l}\text { FF } \\
( \pm 10 \% \text { of } \\
\text { mean } \\
\text { value })\end{array}$ & $\begin{array}{l}\eta(\%) \\
( \pm 5 \% \text { of mean } \\
\text { value })\end{array}$ \\
\hline $\begin{array}{l}\text { CCG- } \\
\text { PEDOT/FTO }\end{array}$ & 827 & 4.37 & 0.60 & 2.16 \\
\hline $\begin{array}{l}\text { CCG-Cl- } \\
\text { PEDOT/FTO }\end{array}$ & 887 & 4.30 & 0.59 & 2.22 \\
\hline CCG on glass & 670 & 3.04 & 0.42 & 0.87 \\
\hline PEDOT/FTO & 722 & 4.93 & 0.60 & 2.12 \\
\hline Bare FTO & 715 & 3.09 & 0.62 & 1.36 \\
\hline $\mathrm{Au} / \mathrm{ITO}$ & 738 & 5.80 & 0.66 & 2.78 \\
\hline
\end{tabular}

Note: Mean values of 4 results for each counter electrode. Errors are standard deviations with 3 degrees of freedom. 
For the efficiencies of the two counter electrodes CCG and CCG-Cl, statistical tests t-tests and F-tests (see "Statistical methods in research and production" by Owen L Davies and Peter Goldsmith pages 75 and 71) were carried out to determine if the differences in their efficiencies (\%) values in Table 5.2 were statistically significant.

\section{F-test}

CCG-PEDOT/FTO: $\eta=2.16 \pm 0.108$, and $n=4$ (separate 4 devices tested)

So the degrees of freedom, $\Phi=\mathrm{n}-1=4-1=3$.

CCG-Cl-PEDOT/FTO: $\quad \eta=2.22 \pm 0.111$, and $n=4$.

So degree of freedom, $\Phi=\mathrm{n}-1=4-1=3$.

t-test for 2 means and $\mathrm{F}$ test for errors

$\mathrm{F}=\mathrm{S}_{1}{ }^{2} / \mathrm{S}_{2}^{2}=(0.111)^{2} /(0.108)^{2}=0.012321 / 0.011664$

Whereas $\mathrm{S}_{1}=$ standard deviation of CCG-PEDOT/FTO

$\mathrm{S}_{2}=$ standard deviation of CCG-Cl-PEDOT/FTO

So, $\mathrm{F}=1.05632716$.

Significances 0.05 (95\%), F value ${ }_{(0.05)}=9.28$

As $\mathrm{F}<\mathrm{F}_{(0.05)}$, standard deviations (SDs) are comparable, and so t-test can now be done.

\section{$\underline{\text { t-test }}$}

$\eta_{1}=2.16 \pm 0.108$

$\eta_{2}=2.22 \pm 0.111$

Variances $=\mathrm{SD}^{2}$.

Combine variances $\mathrm{V}=\left(0.1082+0.111^{2}\right) /(8-2)=3.9975 \times 10^{-3}$

So, $\mathrm{SD}_{\text {combined }}=0.0632$.

$$
\begin{aligned}
& \mathrm{t} \text {-test }=(0.111-0.108) / 0.0632 \sqrt{ }(1 / 4+1 / 4) \\
&=3 \times 10^{-3} / 0.0632 \sqrt{ } 1 / 2 \\
&=4.506 \times 10^{-3}=0.00451 .
\end{aligned}
$$

The total degree of freedom for two samples are 6 (as $\Phi_{1}=3, \Phi_{2}=3$ )

$\mathrm{t}_{(0.05)}=1.94$, so $\mathrm{t}<\mathrm{t}_{(0.05)}$

Therefore, the mean values are not significantly different.

The open circuit voltage $\left(\mathrm{V}_{\text {oc }}\right)$ of the CCG-Cl-PEDOT/FTO counter-electrode device is the highest of the devices tested in this study. Despite the higher $\mathrm{V}_{\mathrm{oc}}$ and comparable FF to a CCG-PEDOT/FTO counter electrode, the CCG-Cl-PEDOT/FTO 
electrode is seen in Figure 5.7 to have only a slightly better efficiency $(\eta \%)$. This is absolutely certain due to the reduced short circuit current, $J_{\mathrm{sc}}$. The relatively low measured short circuit current compared to devices with standard gold counter electrodes may be attributed to any of the following: series resistance of the devices; low optical transmittance; low electron life-time; or poor interfacial charge transfer.

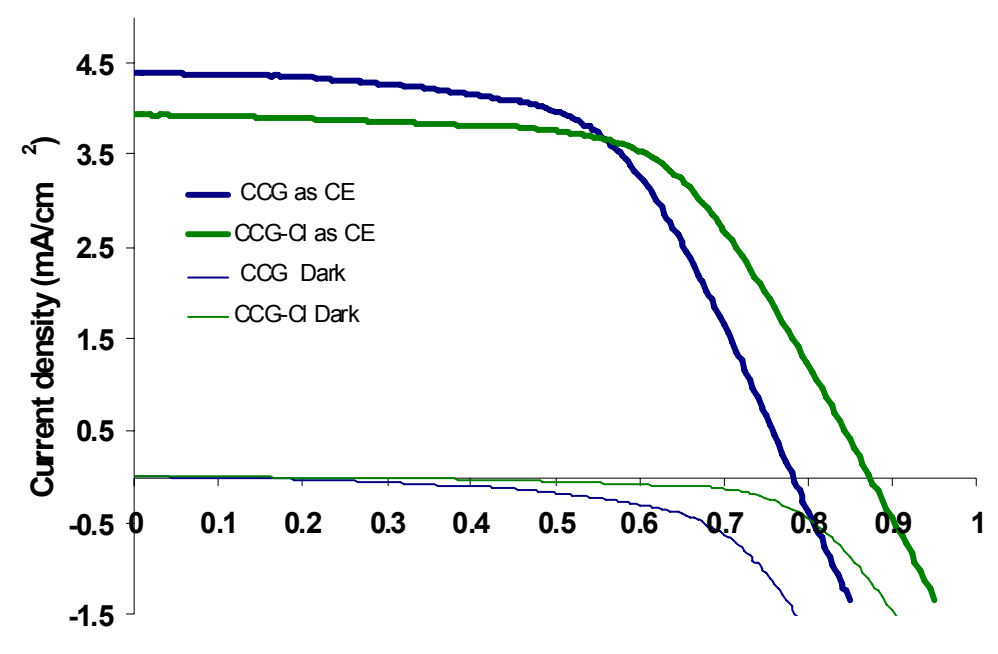

Voltage (V)

Figure 5.7: The current-voltage curves of CCG-PEDOT/FTO (thick blue line) and CCG-Cl-PEDOT/FTO (thick green line) counter electrode solid-state DSCs obtained under one sun illumination (AM 1.5, $\left.\mathrm{P}_{\text {in }} 100 \mathrm{mWcm}^{-2}\right)$.

The solid-state DSC with a CCG-PEDOT/FTO counter-electrode shows a similar trend. The same working electrode with a CCG-Cl-PEDOT/FTO counter-electrode gave slightly lower values for these photovoltaic parameters but the highest $V_{\mathrm{oc}}$ (Table 5.2). This is amongst the highest published in the literature to date for PEDOT-based solid-state dye-sensitized solar cells.

The incident photon to current conversion efficiency (IPCE) was also measured. The IPCE for a cell with a CCG-PEDOT/FTO counter electrode is shown in Figure 5.8. The IPCE shows the effect of the broad absorption band above $600 \mathrm{~nm}$ by Z907 dye. The wavelengths above $700 \mathrm{~nm}$ do not contribute significantly to the photocurrent using Z907 dye. The maximum peak at $\sim 520 \mathrm{~nm}$ is characteristic of the Z907 dye. 
From UV-Vis spectra, it has been observed the dye complex (Z907) is mainly due to metal-to-charge transfer (MLCT) in the visible range whereas ligand centred (LC) $\pi$ $\pi^{*}$ and $n-\pi^{*}$ transition happens in near UV range. This IPCE response is typical of the working electrodes utilised throughout this study and, as expected, the different counter-electrodes do not impact significantly upon the IPCE observed in each case.

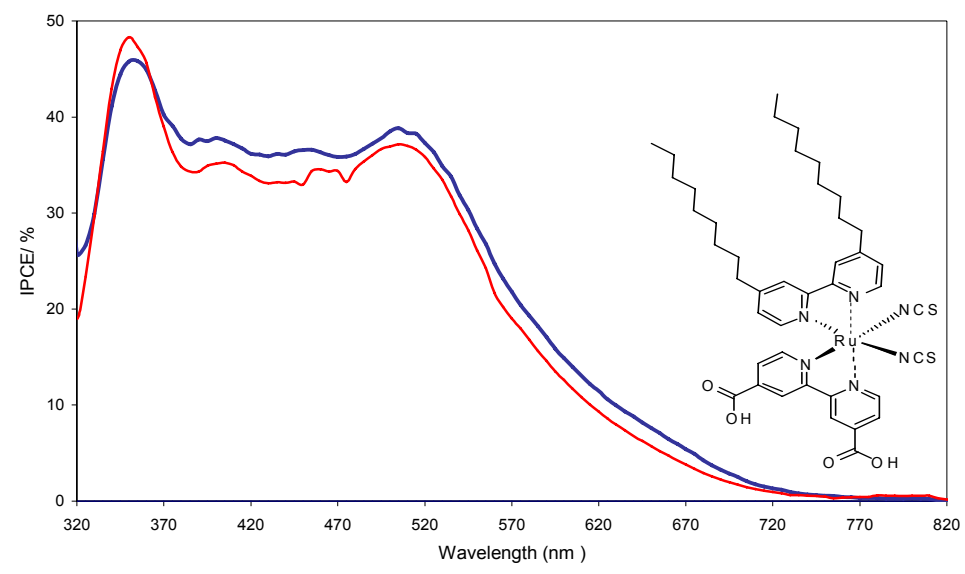

Figure 5.8: Typical IPCE for a cell with CCG-PEDOT/FTO (blue line) and CCG-ClPEDOT /FTO (red line) counter electrodes. Inset: Z907 dye molecule.

The electrode material work function determines whether the electrode forms an ohmic or blocking contact for the charge carrier-electrons in the conduction band of $\mathrm{TiO}_{2}$ and holes in the valance band. The addition of graphene, whose work function is approximately $5 \mathrm{eV}$, will enhance the likelihood of hole transport through the device, which should theoretically increase, the efficiency of the device. The high work function counter electrode material and the interface engineering may play an important role for unidirectional hole extraction during the fabrication of solid state DSCs.

Both chemically converted graphene and chlorine functionalised chemically converted graphene were found to be high performing cost-effective alternatives to conventional precious metals as solid-state dye-sensitized solar cell counter electrodes, highlighting the promise of these materials in commercial production of solid-state DSCs 


\subsubsection{Goretex ${ }^{\circledR}-A u-P E D O T$ based electrode}

The current-voltage performance of solid-state DSCs using Goretex ${ }^{\circledR}$-Au-PEDOT based counter electrodes were evaluated under identical conditions to those for the other electrodes. The solid-state DSCs using the Goretex ${ }^{\circledR}-\mathrm{Au}-\mathrm{PEDOT}$ counterelectrode showed $2.85 \%$ of overall light to power conversion efficiency, which is comparable to an Au/ITO counter-electrode (see summary in Table 5.3 and Figure 5.9). The $2.85 \%$ efficiency results can be attributed to its surface roughness, giving a higher number of physical contact points between photo-anode $(\sim 90 \mathrm{~nm})$ and flexible-compressible counter electrode $(\sim 300 \mathrm{~nm})$ as compared to the rigid and flat gold counter electrode where only a few contact points existed.

However, when the photo-anode (having defects) was used with a rigid, flat Au/ITO counter electrode it showed only $0.85 \%$ efficiency (Figure 5.10). Therefore, while a low current density $\left(3.5 \mathrm{~mA} / \mathrm{cm}^{2}\right)$ and low fill factor $(0.38)$ were found with the $\mathrm{Au} / \mathrm{ITO}$ counter electrode, when the same photo-anode was used with the Goretex ${ }^{\circledR}$ Au-PEDOT counter electrode we achieved a higher current density $\left(6.7 \mathrm{~mA} / \mathrm{cm}^{2}\right)$ and FF (0.65) were achieved (Table 5.3 and Figure 5.10). It was also observed that an extra 70 to $150 \mathrm{mV}$ of open circuit voltage was achieved in each non-PEDOT based counter electrode (Au, Pt, ITO and FTO) which can probably be attributed to the higher work function values of these counter electrodes.

In section 5.3.1, solid-state DSCs were fabricated with up to $2.9 \%$ efficiency using $\mathrm{Au} / \mathrm{ITO}$ counter-electrodes. However, this was achieved reproducibly only when defect-free $\mathrm{TiO}_{2}$ was used. In practice, due to defects, a low degree of reproducibility was generally obtained. The efficiency of $2.9 \%$ is the highest reported in the literature to date for PEDOT based solid-state DSC.

The comparisons between the best current-voltage (JV) results from the two counter electrodes are presented in Figure 5.9. The photo-anode (in Figure 5.9) was defectfree. In Figure 5.9, it is also observed that the performance of the solid-state DSC is comparable when either Goretex ${ }^{\circledR}$-Au-PEDOT or Au/ITO counter electrode was used. 


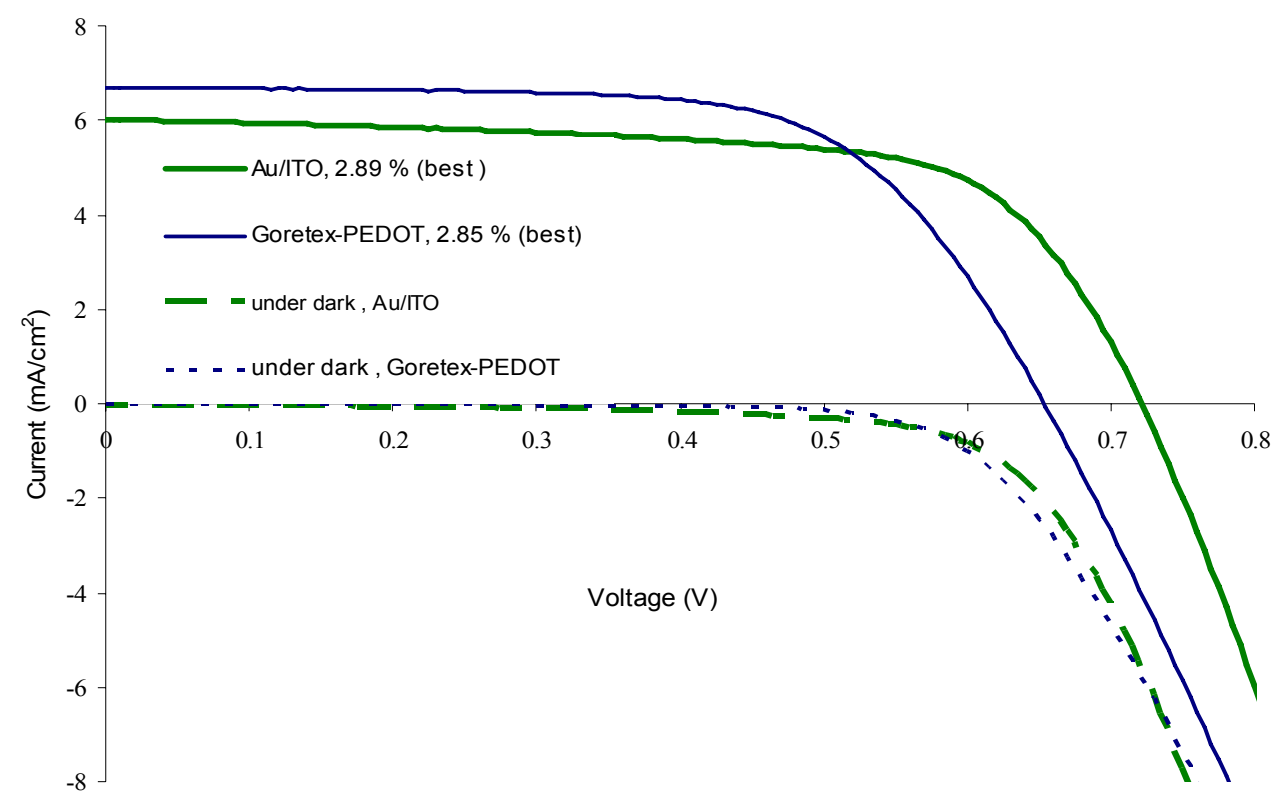

Figure 5.9: Current-voltage characteristics under $100 \mathrm{~mW} \mathrm{~cm}^{-2}$ light intensities (AM 1.5 global) and dark conditions of the two best devices with Goretex ${ }^{\circledR}-\mathrm{Au}-$ PEDOT and Au/ITO counter electrode. A defect-free photoanode was used.

Figure 5.10 presents the current-voltage (JV) curve of the photo-anode assembled with Goretex ${ }^{\circledR}$-Au-PEDOT or Au/ITO counter electrodes. In Figure 5.10, the photoanode which was used had two defects (spikes in its height of its surface profile shown in Figure 5.3a). It is observed that the efficiency of the solid-state DSC with $\mathrm{Au} / \mathrm{ITO}$ counter electrode dropped 30\% compared to Goretex ${ }^{\circledR}$-Au-PEDOT (Table $5.3)$. 


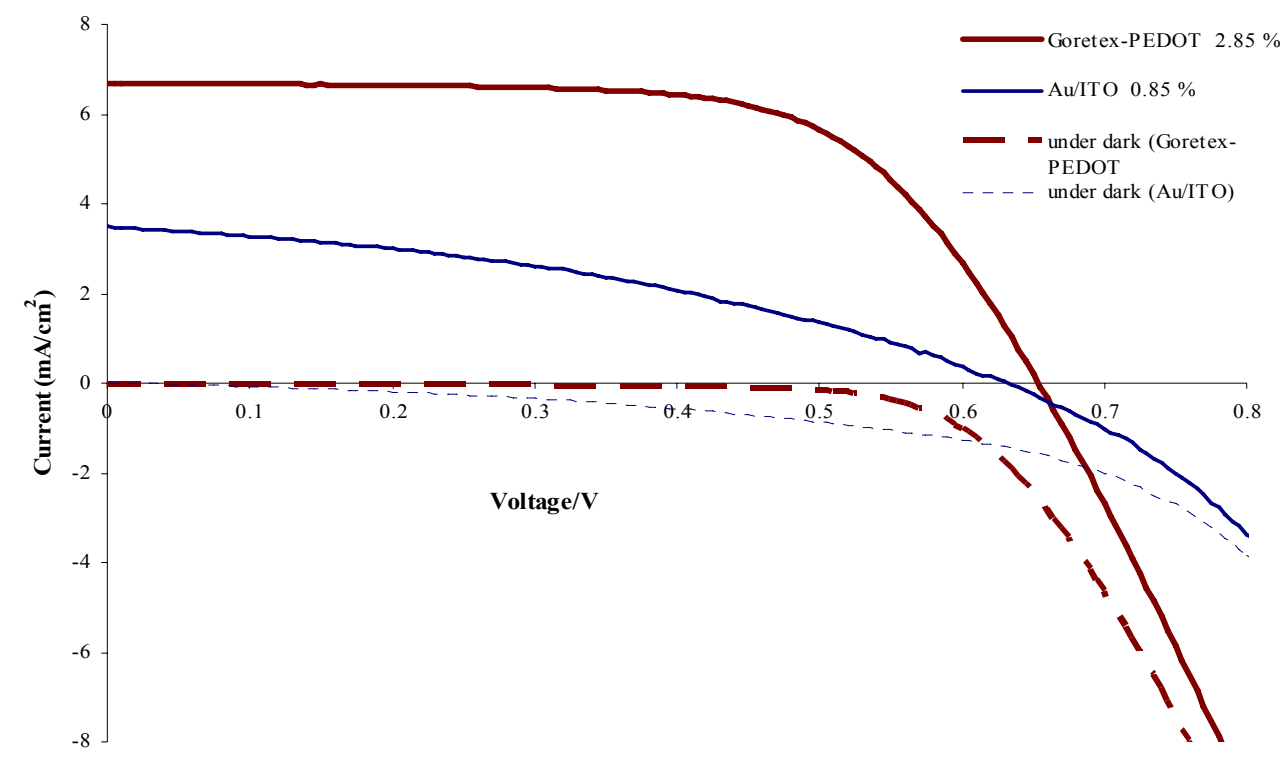

Fig 5.10: Current-voltage characteristics of solid-state DSCs (under $100 \mathrm{~mW} / \mathrm{cm}^{2}$ light intensities (AM 1.5 global and dark conditions) using both Goretex ${ }^{\circledR}$-Au-PEDOT or $\mathrm{Au} / \mathrm{ITO}$ counter electrodes and photo-anodes (with defects).

In a further experiment (Table 5.3) gold was deposited on top of the photo-anode by using of a metal evaporator as described by Bach et al. [10]. However, only $0.5 \%$ efficiency was found for the PEDOT based solid-state DSCs with this gold deposition approach. The low performance achieved with this approach could be due to Au particles being transported through the thin layer of PEDOT causing electrical shorts. The other reason for the low performance using the evaporated Au directly on top of the photo-anode is the lack of a continuous PEDOT layer, which leads to electrical shorts. It may also be due to degradation of the organic layer during the metal evaporation process because of the high vacuum and temperature [35].

Table 5.3 summarises photovoltaic results for a range of different counter electrodes which were prepared by either sputtering or vapour phase polymerization methods. It was found that all electrodes have low surface resistance and are expected to form ohmic contact to the photo-electrochemically deposited PEDOT photo anode. 
Table 5.3: PV performances of solid-state DSCs with different counter electrodes.

\begin{tabular}{|c|c|c|c|c|c|c|c|}
\hline Counter Electrodes & $\begin{array}{c}V_{\mathrm{oc}} \\
(\mathrm{mV})\end{array}$ & $\begin{array}{c}J_{\mathrm{sc}} \\
(\mathrm{mA} \\
\left.\mathrm{cm}^{-2}\right)\end{array}$ & $\mathrm{FF}$ & $\begin{array}{c}\eta \\
(\%)\end{array}$ & $\begin{array}{l}\text { Resistance } \\
(\Omega / \text { square })\end{array}$ & $\begin{array}{c}\mathrm{Ra} \\
(\mathrm{nm})^{\mathrm{c}}\end{array}$ & $\operatorname{Max} \operatorname{dev}(n m)^{d}$ \\
\hline ITO (a) & 795 & 6.11 & 0.58 & 2.81 & 7 & 5 & 25 \\
\hline FTO (a) & 760 & 5.85 & 0.47 & 2.10 & 9 & 8 & 67 \\
\hline $\mathrm{Pt} / \mathrm{ITO}(\mathrm{a})$ & 755 & 6.78 & 0.42 & 2.14 & 10 & 6 & 17 \\
\hline $\mathrm{Au} / \mathrm{ITO}(\mathrm{a})$ & 755 & 6.88 & 0.40 & 2.06 & 2 & 3 & 27 \\
\hline $\mathrm{Au} / \mathrm{ITO}(\mathrm{b})$ & 635 & 3.5 & 0.38 & 0.85 & 2 & 3 & 27 \\
\hline $\begin{array}{c}\text { PEDOT/Au/Goretex } \\
\text { (b) }\end{array}$ & 655 & 6.70 & 0.65 & 2.85 & 6 & 290 & 900 \\
\hline Evaporated Au (ii) & 505 & 0.20 & 0.51 & 0.5 & N.A & $\mathrm{e}$ & $\mathrm{e}$ \\
\hline $\mathrm{Au} / \mathrm{ITO}(\mathrm{a})$ & 725 & 6.02 & 0.66 & 2.89 & 2 & 3 & 27 \\
\hline $\mathrm{TiO}_{2} / \mathrm{Dye} / \mathrm{PEDOT}$ & & & & & & $\begin{array}{c}90 \\
(=40) \\
n m\end{array}$ & $\begin{array}{c}2700(=1400) \\
\mathrm{nm}\end{array}$ \\
\hline
\end{tabular}

Note: a. A defect-free photo-anode; b. An uneven photo-anode (surface profile given in Figure 5.3); c. $\mathrm{R}_{\mathrm{a}}$ is the calculation of average surface roughness of 4 samples (a single scan was done in the middle of a $5 \mathrm{~mm} \times 6 \mathrm{~mm}$ film area; d. Surface roughness calculated on a 1-5 mm scale; e.: Gold is evaporated on top of photo-anode; Therefore Ra (nm) and Max dev (nm) are nearly identical to those for the photo-anode.

Therefore, it is hard to say that their PV performances are limited to only resistive losses. The surface roughness of each counter electrode varies with the surface roughness of photo-anode. Moreover, another factor could be how well the cathode and photo-anode are pressed together. This depends on the porosity, surface roughness, and compressibility of both electrodes. 
From Table 5.3, it can be seen that the open-circuit voltage $\left(V_{\text {oc }}\right)$ is 70 to $150 \mathrm{mV}$ higher for Au, Pt, ITO, or FTO counter electrodes as compared to PEDOT-based electrodes. This may be related to the lower work function of PEDOT.

From the data in Table 5.4 it is clear that Goretex ${ }^{\circledR}-A u-P E D O T$ counter electrode shows good performances even if there was variation in the quality of the photo anode film. Eight devices were fabricated using the same optimal conditions to establish the reproducibility and PV performances of these cells (Table 5.4).

Table 5.4: $\mathrm{PV}$ performances of 8 arbitrarily chosen devices to show the reproducibility achievable with the Goretex ${ }^{\circledR}-\mathrm{Au}-$ PEDOT counter electrode (This Table has been provided as supplementary data in our Langmuir paper [23].

\begin{tabular}{|c|c|c|c|c|c|}
\hline $\begin{array}{c}\text { Sample } \\
\text { ID }\end{array}$ & $\begin{array}{c}\text { Total } \\
\text { Charge } \\
\left(\mathrm{mC} / \mathrm{cm}^{2}\right)\end{array}$ & $V_{\text {oc }(\mathrm{mV})}$ & $\begin{array}{c}J_{\text {sc }} \\
\left(\mathrm{mA} / \mathrm{cm}^{2}\right)\end{array}$ & FF & $\begin{array}{c}\text { Efficiency } \\
(\%)\end{array}$ \\
\hline S-210 D & 14.82 & 700 & 6.42 & 0.67 & 3.01 \\
\hline S-210 D-1 & 14.82 & 680 & 5.96 & 0.71 & 2.86 \\
\hline S-155 D & 16.31 & 675 & 6.02 & 0.66 & 2.63 \\
\hline S-155D-1 & 16.31 & 690 & 5.70 & 0.68 & 2.69 \\
\hline S-188 D & 15.87 & 645 & 7.10 & 0.65 & 2.99 \\
\hline S-188D-1 & 15.87 & 635 & 5.86 & 0.66 & 2.84 \\
\hline S-235 D & 19.48 & 680 & 6.20 & 0.67 & 2.73 \\
\hline S-301 D-1 & 16.40 & 660 & & 0.71 & 2.86 \\
\hline
\end{tabular}

It can be calculated from the data in Table 5.4 that the devices have an average efficiency of $2.8 \pm 0.15 \%$. The variation in efficiencies is due to the different amount of charge developed due to varying amounts of PEDOT in the photo-anode developed by photo-electrochemical polymerization. Another factor may be the 
variation in the quality of clipping between the Goretex ${ }^{\circledR}-A u-P E D O T$ and photoanode.

\section{4 Conclusions}

Metallic counter electrodes lack reproducibility because the surface roughness is not entirely compatible with the working electrode surface. Devices with Goretex ${ }^{\circledR}-\mathrm{Au}-$ PEDOT as counter electrode are found to be more reproducible and more efficient than other counter electrodes used in this Chapter. With PEDOT counter electrodes an average efficiency of $2.66 \%$ was achieved, which is comparable to similar devices using a gold counter electrode. All polymer-based counter electrodes are very good as far as JV reproducibility is concerned.

This novel Goretex ${ }^{\circledR}-A u-P E D O T$ counter electrode, especially, will create an arena for flexible wearable, textile-based photovoltaics which can be integrated into clothes, etc.

The results in this Chapter indicate polymer counter-electrode and graphene-based counter electrode in solid-state DSCs are suitable candidates as cheap alternatives to conventional counter electrodes for photovoltaic devices.

It has also demonstrated here that both chemically converted graphene (CCG) and chlorine functionalised chemically converted graphene (CCG-Cl) can be used as efficient counter-electrodes within solid-state DSCs. These cells show comparable photovoltaic performance parameters to those based on conventional counterelectrodes constructed under similar fabrication conditions, with an overall energy conversion efficiency of $2.22 \%$ observed for the CCG-Cl-PEDOT/FTO counterelectrode. These graphene based devices show significant potential as a costeffective alternative to current precious metal $(\mathrm{Au} / \mathrm{Pt})$ based solid-state DSCs devices. Further investigations are needed in an attempt to optimise the CCG solidstate DSCs cell fabrication design and efficiency and to understand the electrochemical processes involved within these devices. Finally, conclusion, higher 
$V_{\text {oc }}$ is obtained with a graphene counter electrode, whereas a higher $J_{\text {sc }}$ is achieved with the Goretex ${ }^{\circledR}-A u-P E D O T$ and $\mathrm{Au} / \mathrm{ITO}$ counter electrodes, and higher FF achieved with the PEDOT/FTO based counter electrode.

Current work has led to the understanding that the following areas need to be explored.

1. Work needs to be carried out to prepare conducting polymers on flexible substrates to make semi-flexible solid-state dye-sensitized solar cells.

2. Other conducting polymer such as polyaniline will be explored, together with the development of making fully flexible photo-anodes to make flexible devices.

\section{5 References}

1. Grätzel, M., Nature, 414 (2001) 338-344.

2. Regan, B.O., and Grätzel, M., Nature, 353 (1991) 737-739.

3. Sotzing, G.A., Reynolds J.R., and Steel, P.I., Advanced Materials, 9 (1997) 795798.

4. Murakami, T.N., and Grätzel, M., Inorganica Chimica Acta, 361 (2008) 572-580.

5. Saito, Y., Kitamura, T., Wada, Y., and Yanagida, S., Chemistry Letters, 31 (2002) 1060-1061.

6. Murakami, T. N., Ito, S., Wang, Q., Nazeeruddin, M.K., Bessho, T., Cesar, I., Liska, P.R., Baker, H., Comte, P., Péchy, P., and Grätzel, M., Journal of the Electrochemical Society, 153 (2006) A2255-A2261.

7 Saito, Y., Kubo, W., Kitamura, T., Wada, Y., and Yanagida, S., Journal of Photochemistry and Photobiology A: Chemistry, 164 (2004) 153-157.

8 Wei, T. C., Wan, C.C., and Wang, Y.Y., Applied Physics Letters, 88 (2006) 103122-103122-3.

9. Yum, J.H., Chen P., Grätzel, M., and Nazeeruddin, M.K., Chem Sus Chem, 1 (2008) $699-707$.

10. Bach, U., Lupo, D., Comte, P., Moser, J. E., Weissörtel, F., Salbeck J., Spreitzer, H., and Grätzel, M., Nature, 395 (1998) 583-585. 
10a. Snaith, H, J., Moule, A, J., Klein, C., Meerholz, K., Friend, R.H., and Grätzel, M., Nano Letters., 7 (2007) 3372-3376.

10b. Xia, J., Yuan, C., and Yanagida, S., ACS Applied Materials and Interface, 2 (2010) 2136-2139.

11. Wu, J., Li, Q., Fan, L., Lan, Z., Li, P., Lin, J., and Hao S., Journal of Power Sources, 181 (2008) 172-176.

12. MacDiarmid, A.G., Synthetic Metals, 84 (1997) 27-34.

13. Kupila, E.-L., and Kankare, J., Synthetic Metals, 82 (1996) 89-95.

14. Bereznev, S., Kois, J., Golovtsov, I., Opik, A., and Mellikov, E., Thin Solid Films, 511-512 (2006) 425-429.

15. Kitamura, T., Wada, Y., and Yanagida, S., Journal of Photochemistry and Photobiology A: Chemistry, 184 (2006) 234-239.

16. Nagai, H., and Segawa, H., Chemical Communications, 4 (2004) 974-975.

17. Kitamura, T., Maitani, M., Matsuda, M., Wada, Y., and Yanagida, S., Chemistry Letters (2001) 1054-1055.

18. Winther-Jensen, B., Chen J., West, K., and Wallace, G.G., Polymer, 46 (2005) 4664-4669.

19. Park, N.G., and Kim, K., Physica Status Solidi (a), 205 (2008) 1895-1904.

20. Yohannes, T., and Inganäs, O., Solar Energy Materials and Solar Cells, 51 (1998) 193-202.

21. Basse, L., West, K., Winther-Jensen, B., and Jacobsen, T., Solar Energy Materials and Solar Cells, 90 (2006) 341-351.

22. Winther-Jensen, B., and Krebs, F.C., Solar Energy Materials and Solar Cells, 90 (2006) 123-132.

23 Mozer, A.J., Panda, D.K., Gambhir, S., Romeo, T., Winther-Jensen, B., and Wallace, G.G., Langmuir, 26 (2010) 1452-1455.

24. Wang, X., Zhi, L., and Müllen, K., Nano Letters, 8 (2008) 323-327.

25. Gustafsson, G., Cao, Y., Treacy, G. M., Klavetter, F., Colaneri, N., and Heeger, A. J., Nature, 357 (1992) 477-479.

26. Cui, J., Edleman, W.A., Ni, N. L., Lee, J. P., Armstrong, N. R., and Marks, T. J., Advanced Materials, 13 (2001) 1476-1480.

27. Winther-Jensen, B., Winther-Jensen, O., Forsyth, M., and MacFarlane, D.R., Science, 321 (2008) 671-674. 
28. Winther-Jensen, B., Fraser, K., Ong, C., Forsyth, M., and MacFarlane, D.R., Advanced Materials, 22 (2010) 1727-1730.

29. Winther-Jensen, B., and West, K., Macromolecules, 37 (2004) 4538-4543.

30. Winther-Jensen, B., Breiby, D.W., and West, K., Synthetic Metals, 152 (2005) 14.

31. Dennany, L., Wallace, G.G., and Forster, R.J., Langmuir, 25 (2009) 1405314060 .

32. Ahmad, S., Yum, Jun-Ho, Xianxi, Z., Grätzel, M., Butt, H., and Nazeeruddin, M.K., Journal of Materials Chemistry, 20 (2010) 1654-1658.

33. Li, D., Müller, M.B., Gilje, S., Kaner, R.B., and Wallace, G.G., Nature Nanotechnology, 3 (2007) 101-105.

34. Eda, G., Lin, Y.-Y, Miller, S., Chen, C.-W, Su, W.-F., and Chhowalla, M., Applied Physics Letters, 92 (2008) 233305-233305-3.

35. Gunes, S., Neugebauer, H., and Sariciftci, N.S., Fullerenes, Nanotubes, Carbon Nanostructure, 2 (2007) 145-154. 


\section{NON-RUTHENIUM BASED DYES FOR SOLID- STATE DYE-SENSITIZED SOLAR CELLS}




\section{1 Introduction}

Dye-sensitized solar cells (DSCs) provide an alternative to conventional inorganic solar cells, primarily due to their ease of fabrication and the relatively low cost of the materials involved. A wide variety of dyes have been reported for both liquid DSCs (DSCs) and solid-state DSCs (ssDSCs). A range of ruthenium complex dyes, namely N719, N3 and Z907 (shown in Table 6.1) have proven particularly effective since they have broad metal-to-ligand transfer (MLCT) absorption bands covering the near infrared (IR) range. Moreover, the photo-excited states and oxidised forms of the complexes are chemically stable and have redox potentials that enable electron injection into $\mathrm{TiO}_{2}$ and the acceptance of electrons from the $\mathrm{I}^{-} / \mathrm{I}_{3}{ }^{-}$redox couple. Nanocrystalline DSCs with ruthenium based bipyridyl complex dyes (N719, Z907 and N3) as sensitizers have received considerable attention and achieved efficiencies of up to $11 \%[1-3]$.

Ruthenium is one of the rarest elements as shown in Figure 6.1 and mass production of solar cells may lead to significant price increase due to scarcity of this element. The production of devices based on a ruthenium dye is therefore challenging. Grätzel and co-workers recently reported that DSCs can be fabricated with metal-free organic dyes and have an efficiency of up to $9 \%$ [4-5]. Alternative dyes such as triphenylmethane, xanthanene, perylenes, and coumarin have consequently been explored (see Chapter 1). The criteria for a dye to be an efficient photo-sensitizer were outlined in Section 1.2.3.3 (Chapter 1). 


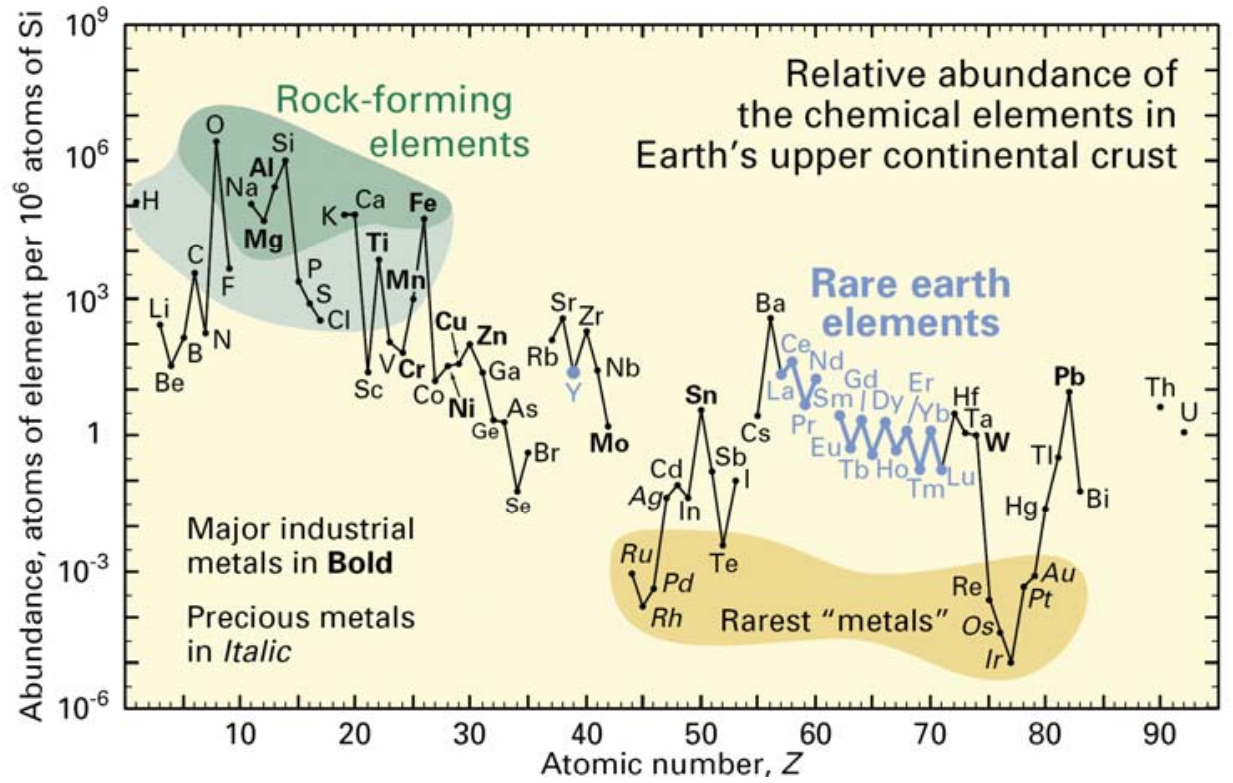

Figure 6.1: Abundance of different elements that are of interest to solar cell technologies (U.S Geological Survey Fact Sheet 087-02).

In DSCs, researchers use the redox couple $\mathrm{I}^{-} / \mathrm{I}_{3}{ }^{-}$with a volatile solvent, which is not good for practical outdoor applications. The long term stability and durability of such devices at operational conditions $\left(85^{\circ} \mathrm{C}\right.$ thermal and $85 \%$ humidity) is a concern whenever any new photovoltaic technology is considered for commercial purposes $[6,11]$. While DSCs are typically less expensive than conventional silicon solar cells, several inherent issues with them must be addressed. This includes volatility of the electrolyte, sealing, thermal stability towards high temperature, and corrosive nature of the electrolyte.

In this chapter, the dyes shown in Table 6.2 will be explored for use in ssDSCs except P-44 dye. This is the first study on the application of non-ruthenium based dyes for solid-state DSCs with photo-electrochemically deposited PEDOT as the hole transporting material. Non-ruthenium dyes have not been reported as photosensitizers for PEDOT based ssDSCs in the literature so far. 
Table: 6.1: Various ruthenium-based dyes used to date in DSCs.
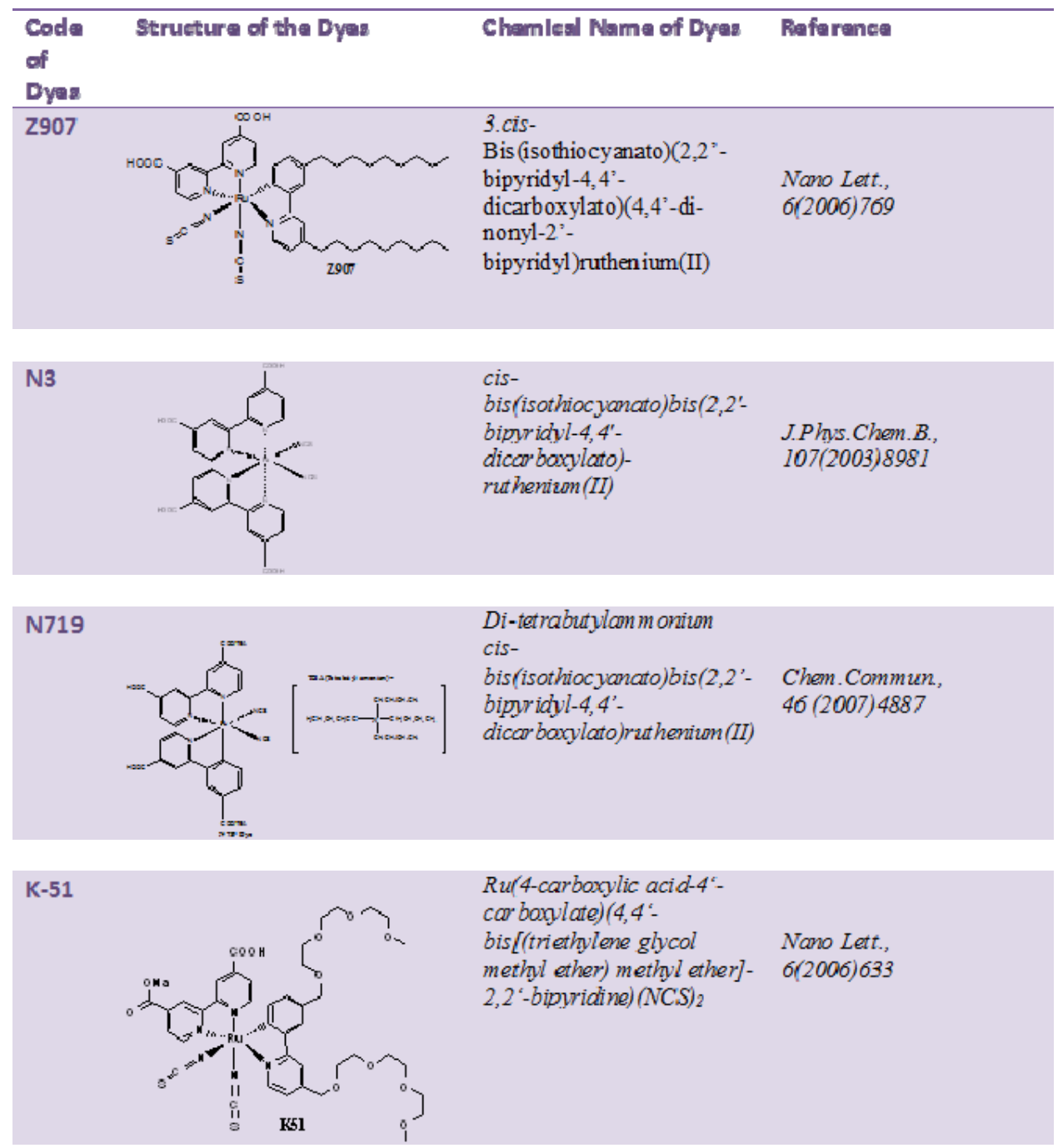

Ru(4-carboxylic acid-4:cor boxylate) $(4,4$ : bis[(triethylene glycol Nano Lett. methyl ether) methyl ether]- $6(2006) 633$ $2,2^{\circ}$-bipyriaine) $(\mathrm{NCS})_{2}$

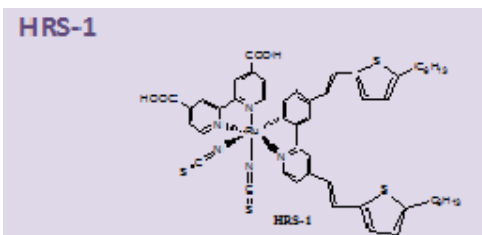

cis-Ru-4, 4-ci-

hexythinelvinyl-2,2-

bipyridyl- Chem.Commun

4,4-dicarboxylic aci d-2,2- 23(2006) 2460 bipyridyl-NCS-2 
Table 6.2: List of various non-ruthenium based dyes used to date in DSCs.

\begin{tabular}{l}
$\begin{array}{l}\text { Code } \\
\text { of } \\
\text { Dyes }\end{array}$ \\
\hline GD2
\end{tabular}
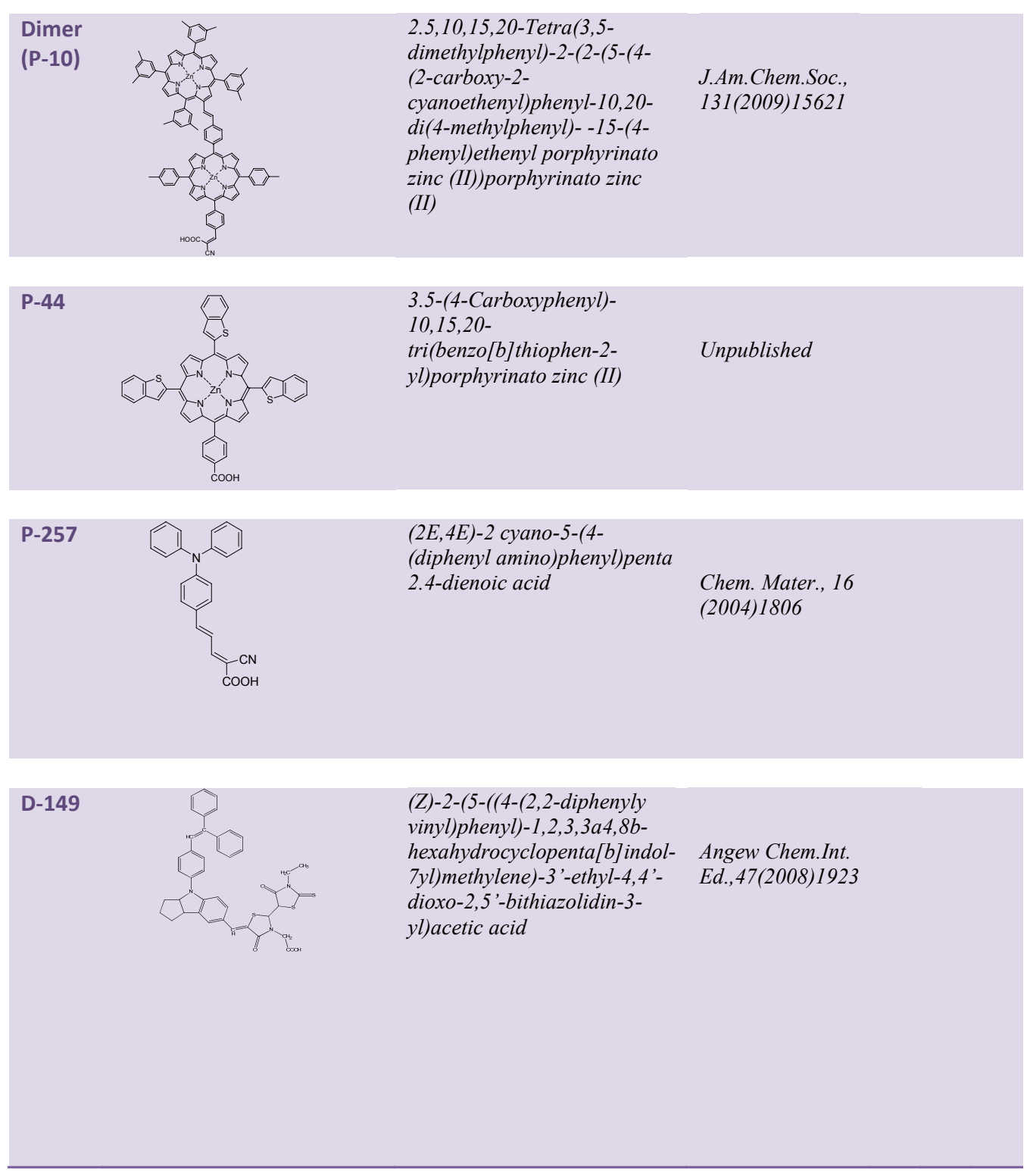


\subsubsection{Zinc-porphyrin dyes}

Zinc-porphyrin dyes (such as GD2, dimer (P-10) and P-44) are excellent light absorbers (Table 6.2), thus making them interesting candidates for use as light harvesters in DSCs [12]. Zinc-porphyrin has been used to successfully produce DSCs with up to $6 \%$ as fabricated by Mozer et al. $[13,14]$ and therefore was considered as a sensitizer in this study.

Many DSC-specific porphyrins have been synthesised by different groups [15-17]. Because of their intense absorption in the Soret and Q bands, light harvesting occurs over a wide spectral region. Some zinc-porphyrin dyes have provided promising candidates for ssDSCs [18-20].

The attachment of a large porphyrin array to a nano-crystalline semiconductor surface provides a method to dramatically increase the surface dye concentration, resulting in an increase in the light energy conversion efficiency of the device to $7 \%$. Zinc porphyrin has been found to be an efficient and potentially low-cost dye [1924].

\subsubsection{P-257 dye as a sensitizer}

The application of the P-257 dye (Table 6.2) in ssDSC has been investigated in this Chapter. This relatively small organic dye has been used by Yanagiada et al. for DSCs [25]. It has a higher molar extinction coefficient than Z907 dye. Also the oxidation potential of this dye has higher $\sim 0.4 \mathrm{~V}$ oxidation potential than the Z907 dye [10] which is good for successful in situ photo-electrochemical polymerization. But the preliminary experimentally findings by transient absorption spectroscopy reported that the higher oxidation potential of this dye did not help in accelerating the regeneration kinetics significantly [10]. 


\subsubsection{D-149 dye as photo-sensitizer}

D-149 (Table 6.2) dye has a high molar extinction coefficient. The absorption maximum for D-149 in tert-butyl alcohol is: $\lambda_{\max }=526 \mathrm{~nm}, \epsilon=68,700 \mathrm{M}^{-1} \mathrm{~cm}^{-1}$. In DSCs, this dye shows an IPCE of more than $80 \%$ over the wavelength range 500-900 $\mathrm{nm}$, thus allowing it to harvest more photons than the ruthenium-based dye Z907. Recently, D-149 dye has been used for fabrication of devices using the organic holetransporting medium (HTM) 2, 2c, 7, 7c-tetrakis-( $N, N$-di-p-methoxyphenyl-amine)9,9c-spirobifluorene (spiro-OMeTAD) [26-29]. Three organic indoline-based sensitizer dyes with high molar extinction coefficients (namely D-102, D-120, and D-149) were investigated. Differences in electron lifetime for each dye were attributed to the dipole moment at the $\mathrm{TiO}_{2} /$ spiro-OMeTAD interface. This shifts the $\mathrm{TiO}_{2}$ conduction band energy relative to the Fermi energy level of the hole transporting material, thus affecting the open circuit voltage $\left(V_{\text {oc }}\right)$ and the current density $\left(J_{\mathrm{sc}}\right)$.

Others have fabricated ssDSCs using indoline dyes and $\mathrm{p}-\mathrm{CuI}$ as the hole conductor (p-type inorganic material) and achieved up to 3.6\% efficiency (which is comparable with the N3 dye used in ssDSCs with $\mathrm{CuI}$ ). The redox potential for spiro-OMeTAD is $0.55 \mathrm{~V}$ vs. $\mathrm{Ag} / \mathrm{AgCl}$, which is $0.45 \mathrm{~V}$ more positive than the redox potential of $\mathrm{I}^{-}$ $/ \mathrm{I}_{3}{ }^{-}$redox-couple used in DSCs. Therefore in principle, spiro-OMeTAD cells should give higher open circuit voltages. The HOMO-LUMO level of each dye presented in Tables 3.7 and 3.8 of Chapter 3 give a better understanding of the suitability of the dye for effective photo-electrochemical polymerization. It is important to mention here that the bis-EDOT oxidation potential should be lower than the oxidation potential of the respective dyes for oxidation towards photo-electrochemical polymerization. PV performances of liquid DSCs with different dyes with $\mathrm{I}^{-} / \mathrm{I}_{3}{ }^{-}$redox couple reported in various journals are presented in Table 6.3. 
Table 6.3: PV performances of liquid DSCs with different dyes with $\mathrm{I}^{-} / \mathrm{I}_{3}{ }^{-}$redox couple reported in the literature.

\begin{tabular}{|c|c|c|c|c|c|}
\hline $\begin{array}{l}\text { Name of } \\
\text { the dyes }\end{array}$ & $\begin{array}{l}V_{o c} \\
(m V)\end{array}$ & $\mathrm{J}_{\mathrm{sc}}\left(\mathrm{mA} / \mathrm{cm}^{2}\right)$ & FF & $\begin{array}{l}\text { Efficiencies } \\
(\%)\end{array}$ & References \\
\hline Z907 & 666 & 15.2 & 0.66 & 6.7 & Nano Lett., 6 (2006)769-773 \\
\hline N3 & 630 & 19.5 & 0.65 & 7.4 & $\begin{array}{l}\text { J. Phys. Chem. B., 107(2003) } \\
8981\end{array}$ \\
\hline N-719 & 769 & 19.9 & 0.65 & 10.1 & $\begin{array}{l}\text { Chem. Commun., } 46 \text { (2007) } \\
4887\end{array}$ \\
\hline K-51 & 715 & 16.6 & 0.67 & 8.1 & Nano Lett., 6 (2006) 633 \\
\hline HRS-1 & 680 & 20 & 0.69 & 9.5 & Chem.Commun.,23(2006) 2460 \\
\hline GD2 & 626 & 9.09 & 0.69 & 3.9 & Chem. Commun., 39 (2008) 4741 \\
\hline $\begin{array}{l}\text { Dimer } \\
\text { (P-10) }\end{array}$ & 715 & 8.04 & 0.65 & $\begin{array}{l}3.8 \\
\text { (thin } \mathrm{TiO}_{2} \\
\text { film) }\end{array}$ & $\begin{array}{l}\text { J. Am. Chem. Soc., 131(2009) } \\
15621\end{array}$ \\
\hline D-149 & 707 & 12.5 & 0.72 & 6.38 & $\begin{array}{l}\text { Angew Chem. Int. Ed., 47(2008) } \\
1923\end{array}$ \\
\hline P-257 & 730 & 11.1 & 0.66 & 5.3 & Chem. Mater., 16 (2004) 1806 \\
\hline
\end{tabular}




\section{2 Experimental}

\subsubsection{Dye loading}

\subsubsection{Zinc-porphyrin dye}

Firstly, $3 \mu \mathrm{m}$ thick $\mathrm{TiO}_{2}$ films were sintered for $2 \mathrm{hrs}$ to remove water, and then immersed immediately in the Zn-porphyrin GD2 dye for $2 \mathrm{hrs}$. UV-Vis spectra were taken to investigate the dye loading of GD2 dye on the $\mathrm{TiO}_{2}$ films. These loading measurements were corroborated by measuring the concentration of dye that could be desorbed from the same films. The Zn-porphyrin dye was desorbed from sensitized films by immersing them in $4 \mathrm{ml}$ of $0.01 \mathrm{M}$ tetrabutylammonium hydroxide (TBAOH.30 $\mathrm{H}_{2} \mathrm{O}$ ) in THF. UV-Vis spectra of films were made by placing a glass coverslip on the film then injecting one drop of propylene carbonate between the film and the coverslip to better match their refractive index and remove interference fringes from the baseline. A UV-Vis spectrum of each desorbed solution was taken in $1 \mathrm{~cm}$ pathlength cuvettes. The base-line for dye desorption was corrected to the respective desorption solution prior to immersion; in the case of sensitized films, sintered $\mathrm{TiO}_{2}$ films were used as base line.

\subsubsection{P-257 dye}

Similarly, the blocking $\mathrm{TiO}_{2}$ layer and mesoporous $\mathrm{TiO}_{2}$ layer on the FTO anode were prepared using the standard method discussed in Chapter 2. The sintered electrode was then immersed into $0.4 \mathrm{mM} \mathrm{P-257}$ dye and $0.8 \mathrm{mM}$ chenodeoxycholic (DCA) as co-adsorbent in acetonitrile and t-butanol (1:1 by volume) and kept for sensitization for $20 \mathrm{hrs}$. The structure of DCA is presented in figure 6.2. Sensitization was also done without mixing any co-adsorbent in the dye solution. 


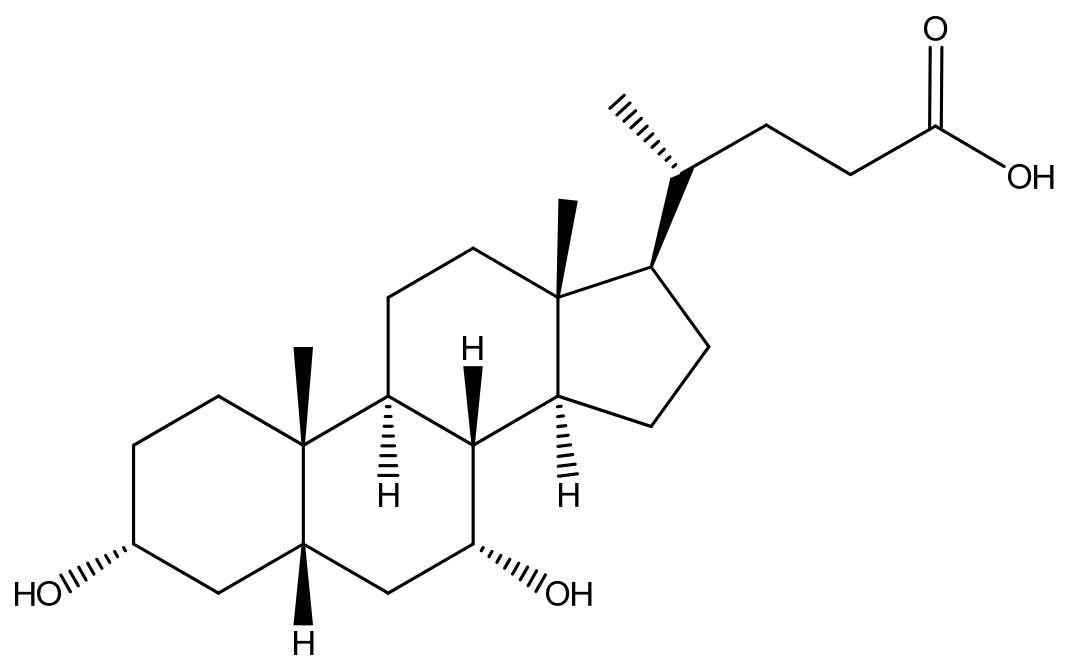

Figure 6.2: Chenodeoxycholic acid

\subsubsection{D-149 Indoline dye}

The photo-anode was prepared by following the general method described in section 6.2.1.2. The sintered electrode was immersed into $0.4 \mathrm{mM}$ D-149 dye and $0.8 \mathrm{mM}$ DCA in acetonitrile and t-butanol (1:1 by volume) and kept for sensitization (for 20 hrs).

\subsubsection{Calculation of HOMO-LUMO levels}

HOMO-LUMO levels and band gap energy were calculated using the experimental data from UV-Visible spectra as well as cyclic-voltammograms (CVs). The calculated values were used to establish a reliable procedure for rapid screening of suitable photo-sensitizing dyes/ monomers for photo-electrochemical deposition. The HOMO value of bis-EDOT suggested that it could be photo-polymerized on a dyed $\mathrm{TiO}_{2}$ film.

To investigate the electrochemical properties of bis-EDOT and GD2 dye with and without illumination, $\mathrm{CV}$ measurements were carried out in a three-electrode electrochemical cell containing $0.10 \mathrm{M} \mathrm{LiClO}_{4}$ acetonitrile solution, and with a $1 \mathrm{~cm}^{2}$ 
area of the dye $/ \mathrm{TiO}_{2} /$ FTO serving as the working electrode. Platinum foil was used as a counter electrode with a $\mathrm{Ag} / \mathrm{AgCl}$ reference electrode, with an eDAQ electrochemical system used to conduct and record the CVs.

\subsubsection{Dye desorption from the photo-anode}

Same procedure followed as in section 6.2.1.2.

\subsubsection{Zinc-porphyrin dye}

The dye desorption experiment was undertaken in order to calculate the amount of dye on the surface of $\mathrm{TiO}_{2}$ photo-anode. Zn-porphyrin dye was desorbed from sensitized films by immersing them a film in $4 \mathrm{ml}$ of $0.01 \mathrm{M}$ TBAOH.30 $\mathrm{H}_{2} \mathrm{O}$ in THF. The thickness of the $\mathrm{TiO}_{2}$ film was $3 \mu \mathrm{m}$ (measured by surface profilometer). A UV-Vis spectrum of each desorbed solution was taken. Baseline correction was carried out as described in section 6.2.1.1.

\subsubsection{P-257 dye}

P-257 dye films of $3 \mu \mathrm{m}$ thickness were immersed into a $10 \mathrm{ml}$ solution of $0.1 \mathrm{ml}$ $\mathrm{NaOH}$ in 1:1 EtOH: $\mathrm{H}_{2} \mathrm{O}$ to desorb the dye from the respective films. UV-Vis spectra of each desorption solution were recorded (as above) to determine the amount of dye desorbed.

\subsubsection{Preparation of working electrode by in-situ photo-electrochemical polymerization}

Working electrode preparation was the first fabrication step. A $\mathrm{TiO}_{2}$ blocking layer approximately $100 \mathrm{~nm}$ thick was applied by spin coating Ti-isopropoxide solution on the FTO glass substrate and then sintering at $500^{\circ} \mathrm{C}$ for 30 min. A transparent 
mesoporous $\mathrm{TiO}_{2}$ film of $\sim 5.5 \mu \mathrm{m}$ thickness was then made on top of the blocking layer by doctor blading Solaronix Nanoxide-T paste and sintering for a further 30 min at $500^{\circ} \mathrm{C}$. The mesoporous film was sensitized by 2 hour immersion in GD2 dye dissolved in THF. The scheme for photo-electrochemical polymerization is shown in Figure 6.3.

To form the hole transport material (HTM), dyed $\mathrm{TiO}_{2}$ substrates were put in an electrochemical cell containing ACN solution of $0.10 \mathrm{M} \mathrm{LiClO}_{4}$ and $0.01 \mathrm{M}$ bisEDOT and immediately the substrates were then illuminated from the mesoporous $\mathrm{TiO}_{2}$ side by a lamp (Figure 6.3a). The light intensity was varied from $3.2 \mathrm{~mW} / \mathrm{cm}^{2}$ to $25 \mathrm{~mW} / \mathrm{cm}^{2}$ using appropriate optical filters. Polymerization was carried out under potentiostatic conditions in a three-electrode, one-compartment electrochemical cell. The dye-adsorbed $\mathrm{TiO}_{2}$ substrate was the working electrode, Pt mesh was the counter electrode, and $\mathrm{Ag} / \mathrm{AgCl}$ the reference electrode. A constant potential of $0.2 \mathrm{~V}$ versus $\mathrm{Ag} / \mathrm{AgCl}$ was applied and the current monitored using e-DAQ software. PEDOT was subsequently deposited onto the mesoporous $\mathrm{TiO}_{2}$ film. The total charge transferred during polymerization was calculated by integration of the polymerization current over time. The whole ssDSC structure is shown in figure $6.3 \mathrm{~b}$.

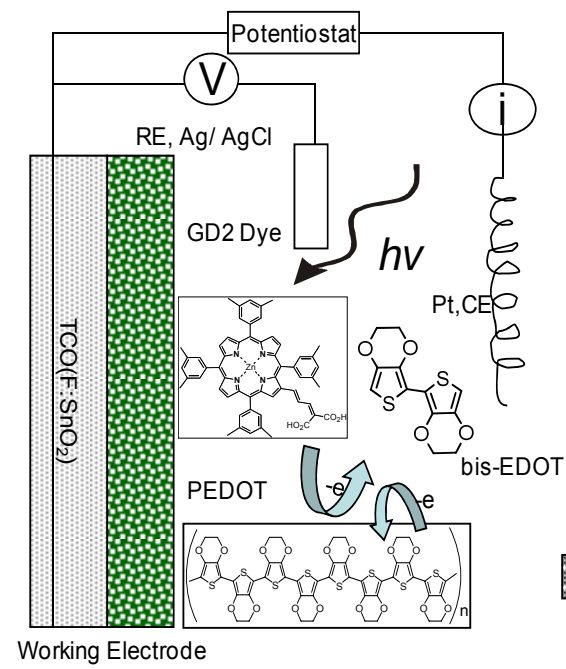

(a)

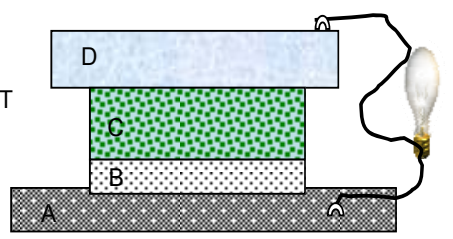

(b)

Figure 6.3: (a) Schematic of photo-electrochemical polymerization of bis-EDOT to PEDOT via zinc-porphyrin $\left(\mathrm{GD}_{2}\right)$ dye. (b) The device structure is illustrated. A: Fluorine-doped $\mathrm{SnO}_{2}$ glass substrate; B: Blocking $100 \mathrm{~nm} \quad \mathrm{TiO}_{2}$ layer; C: Mesoporous $\mathrm{TiO}_{2}$-dye-PEDOT; D: Goretex ${ }^{\circledR}-\mathrm{Au}-\mathrm{PEDOT}$. 
The P-257 dye-sensitized titania layer was coated with a photo-polymerized film of PEDOT. To form the hole transport material (HTM), substrates were immersed in a solution of $0.10 \mathrm{M} \mathrm{LiClO}_{4}$ and $0.01 \mathrm{M}$ bis-EDOT in a range of solvents such as acetonitrile, THF, and 3-methoxypropionitrile (MP), and illuminated from the mesoporous $\mathrm{TiO}_{2}$ side by a Solartron lamp within an intensity range of 2.5-45 $\mathrm{mW} / \mathrm{cm}^{2}$.

The same method was followed for the D-149 dye as for the P-257 dye. Different solvents such as MP and THF were considered in order to prevent the desorption of D-149 dye during the photo-electrochemical polymerization method. The light intensity was varied using optical neutral density filters. Photo-electrochemical polymerization was carried out under potentiostatic conditions in a three-electrode, one-compartment electrochemical cell, as described above for porphyrin dyes.

\subsubsection{Device fabrication of solid-state dye-sensitized solar cells}

After polymerization, the photo-anode films were rinsed with acetonitrile and treated with 1-ethyl-3-methylimidazolium bis-trifluoromethanesulfonimide (EMI.TFSI) containing 0.20 M lithium bis-trifluoromethanesulfonimide (LiTFSI) and 0.20 M 4tert-butylpyridine (t-BP) for $24 \mathrm{hrs}$. The sandwich-type devices were completed by clipping the conducting polymer counter electrode (Goretex $\left.{ }^{\circledR}-A u-P E D O T\right)$ to the photo-anode.

Transmission was characterised by UV-visible spectra (Shimadzu UV-1601 spectrophotometer), $\mathrm{TiO}_{2}$ film thickness by surface profilometry (Veeco Dektak 150 ), and conductivity of the PEDOT on Goretex ${ }^{\circledR}$ by four-point probe (Jandel RM3). UV-Vis spectra of dyes adsorbed onto $\mathrm{TiO}_{2}, \mathrm{PEDOT} /$ dyed $\mathrm{TiO}_{2}$ were also taken using the Shimadzu UV-1601 spectrophotometer.

Liquid DSCs were also fabricated in this study using zinc-porphyrin dye and P257 dye through standard procedures in order to compare with the PV performances of the solid-state dye-sensitized solar cells 


\subsubsection{Device fabrication of liquid dye-sensitized solar cells using phenyl- conjugated Oligoene P-257 sensitizers}

The purpose of this experiment was to replace the hole transporting material PEDOT and use $\mathrm{I}^{-} / \mathrm{I}_{3}{ }^{-}$electrolyte as a redox mediator in DSCs, in order to compare the PV performance of the liquid DSCs with ssDSCs.

\section{3 Results and discussion:}

\subsubsection{Dye loading of Zinc-porphyrin GD2 Dye}

The HOMO/ LUMO values of the zinc-porphyrin dyes in solution were found here to be slightly different to those when bound to $\mathrm{TiO}_{2}$. The difference in these values is due to the higher concentration of dye in the film. The zinc-porphyrin dyes such as GD2 were found to be suitable for the photo-electrochemical deposition of PEDOT in ssDSCs. Figure 6.4 shows that the GD2 dye surface coverage on mesoporous $\mathrm{TiO}_{2}$ film was $1.83 \times 10^{-8}$ moles $/ \mu \mathrm{m}^{3}$ (Abs: $\lambda_{\max }=436 \mathrm{~nm}, \varepsilon=122600 \mathrm{M}^{-1} \mathrm{~cm}^{-1}$ ), which is similar to values reported in the literature [14]. The absorption spectrum of GD2 dye was recorded in THF solution and compared to the dye adsorbed into a $\sim 3 \mu \mathrm{m}$ thick $\mathrm{TiO}_{2}$ film (Figures 6.4 and 6.5, respectively). In solution, absorption bands were observed at $440 \mathrm{~nm}$ and $620 \mathrm{~nm}$ due to $\pi$ - $\pi^{*}$ absorption of the conjugated macrocyclic porphyrin. An intense Soret band and low intensity Q bands were found at $436 \mathrm{~nm}, 570 \mathrm{~nm}$ and $620 \mathrm{~nm}$, respectively. The visible absorption spectrum of GD2 dye desorption solution (anchored on $\mathrm{TiO}_{2}$ film) (Figures 6.5) shows similar features to that in solution. 


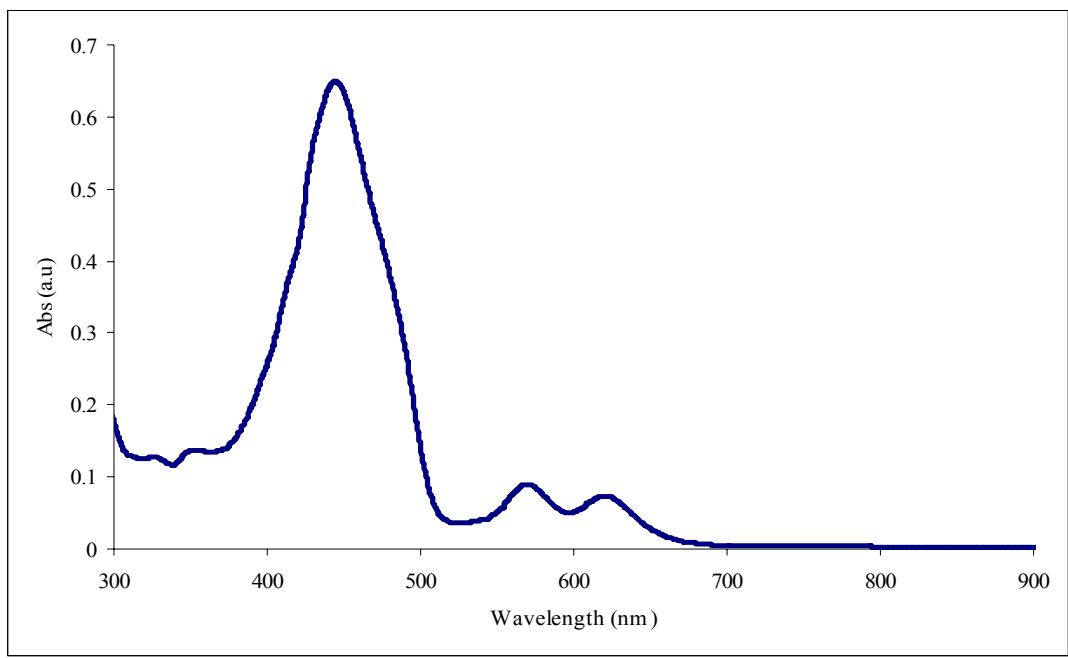

Figure 6.4: UV-Vis spectrum of GD2 dye in THF solution.

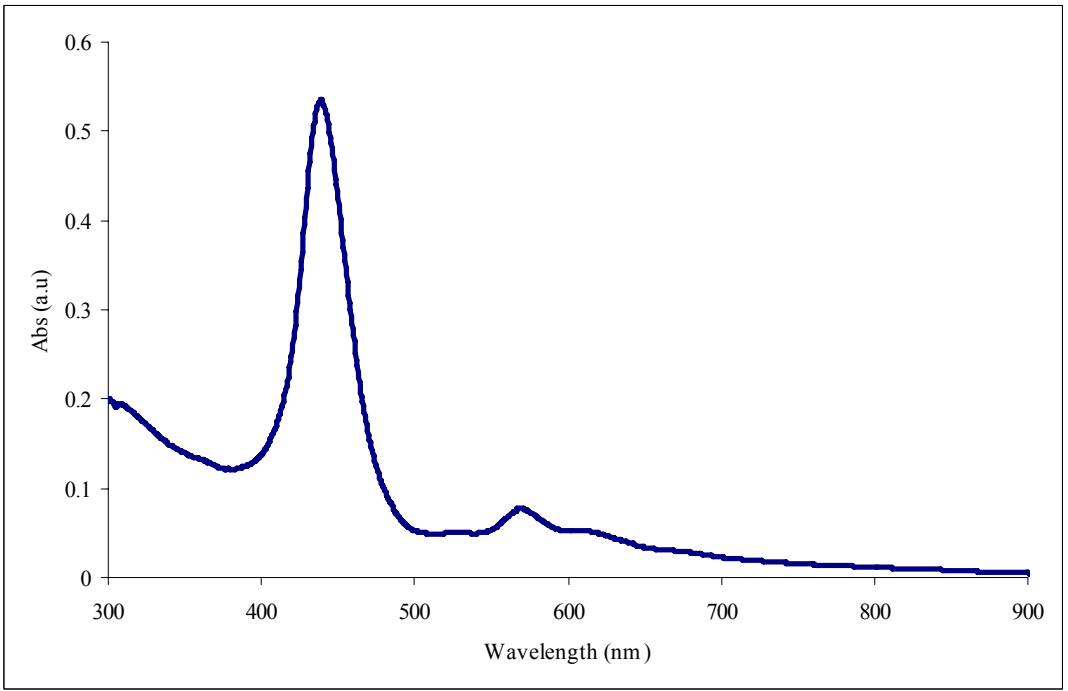

Figure 6.5: UV-Vis spectrum of GD2 dye desorption solution.

\subsubsection{Dye loading of P-257 dye}

\subsubsection{Calculation of the amount of $\mathrm{P}-257$ dye on $\mathrm{TiO}_{2}$ surface}

The UV-Vis spectra of P-257 dye solution and dye desorption (from sensitised $\mathrm{TiO}_{2}$ film) are shown in Figures 6.6 and 6.7 respectively. The absorption intensity at the 
$410 \mathrm{~nm}$ peak in solution of P-257 dye was 0.946 (Figure 6.6) where as in the dye desorption absorption was 0.212 (Figure 6.7).

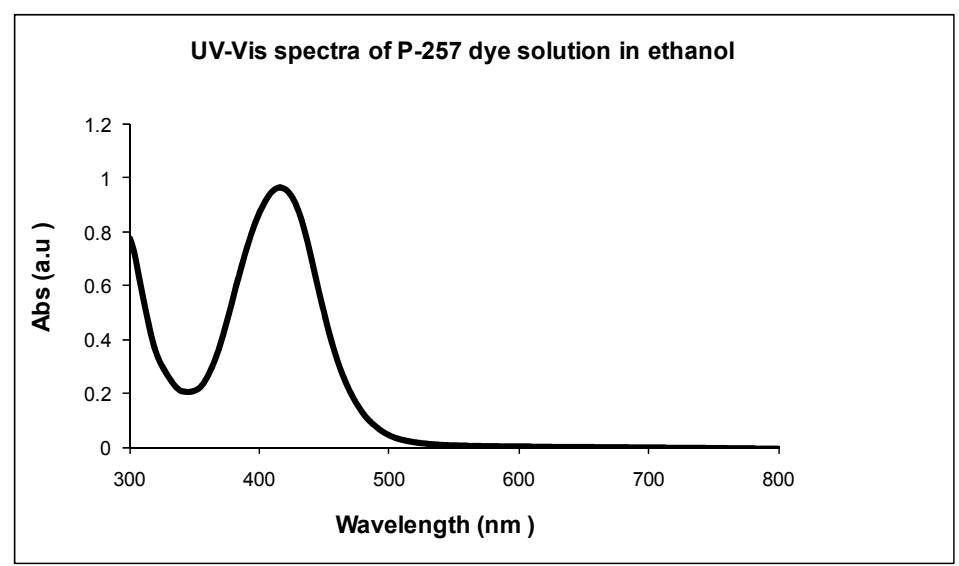

Figure 6.6: UV-Vis spectrum of $0.04 \mathrm{mM}$ of P-257 dye solution in ethanol.

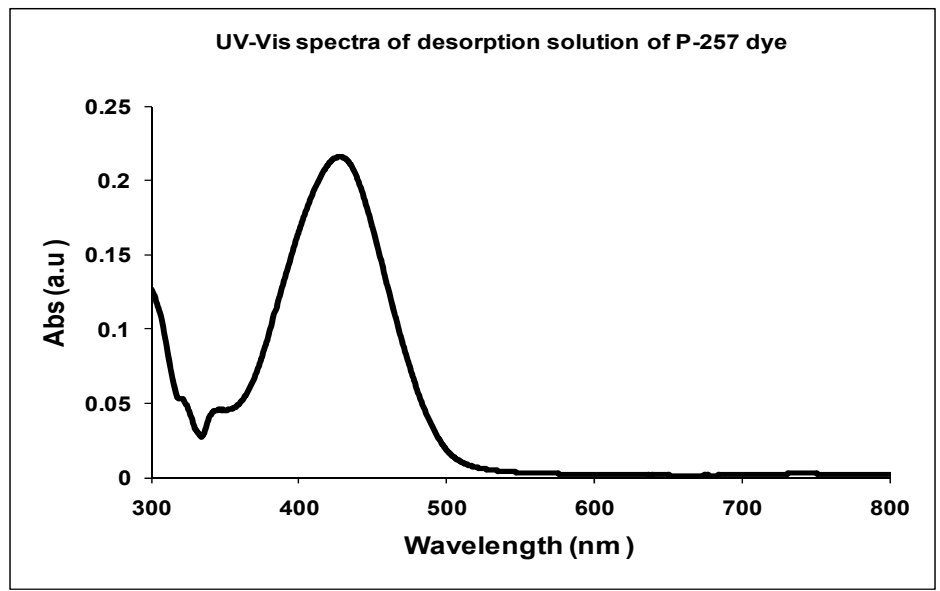

Figure 6.7: UV-Vis spectrum of $0.4 \mathrm{mM}$ P-257 dye desorption solution.

The molar extinction coefficient of the P-257 dye at $416 \mathrm{~nm} \lambda_{\max }$ was calculated from the UV-Vis data in Figure 6.6 as $24,050 \mathrm{M}^{-1} \mathrm{~cm}^{-1}$

The surface coverage for the P257 dye

So dye Abs at $416 \mathrm{~nm}=0.225$ (Figure 6.7).

$\mathrm{A}=\varepsilon c$, so $c=\mathrm{A} / \varepsilon=0.225 / 24,050$

So $c_{\mathrm{P}-257}=8.52 \times 10^{-6}$ moles $/ \mathrm{L}$

Therefore, number of moles, $\eta_{\mathrm{P}-257=C_{\mathrm{P}-257}} \times \mathrm{V}_{\text {desorption, }}$ 
Where $\mathrm{V}_{\text {desorption }}=$ the volume in which the dye was desorbed from the $\mathrm{TiO}_{2}$ film. So $\eta_{\mathrm{P}-257}=8.52 \times 10^{-6}$ moles $/ \mathrm{L} \times 0.01 \mathrm{~L}$

$$
=8.52 \times 10^{-8} \text { moles }
$$

Hence, Surface coverage of dye in $\mathrm{TiO}_{2}$ film, $\Gamma=\eta /$ (Area $\times$ thickness of $\mathrm{TiO}_{2}$ film)

$$
\begin{aligned}
\Gamma_{\mathrm{P}-257} & =8.52 \times 10^{-8} \text { moles } / 0.72 \times 3 \\
& =3.9 \times 10^{-8} \mathrm{moles} / \mu \mathrm{m}^{3}
\end{aligned}
$$

N.B: The thickness of the $\mathrm{TiO}_{2}$ film was $3 \mu \mathrm{m}$, and the area of the film from which dye was desorbed was $8 \mathrm{~mm} \times 9 \mathrm{~mm}$ (i.e. $0.72 \mathrm{~cm}^{2}$ ), and the volume in which desorbed was $0.01 \mathrm{~L}$.

\subsubsection{Calculation of the amount of P-257 dye desorbed from the photo-anode during the photo-electrochemical deposition of PEDOT.}

UV-Vis spectra of each dye desorption solution were taken after the photoelectrochemical deposition of PEDOT. During the photo-electrochemical polymerization (PEP) it was observed that the colour of the electrolyte changed to a red colour, consistent with some of dye desorbing from photo-anode in the electrochemical cell. The UV-Vis spectra of the desorption solutions where the PEP was carried out under various conditions, such as different periods of PEDOT growth, different applied potentials, different light intensities, under dark electrochemical polymerization and under light irradiation, are shown in Figure 6.8 and Figure 6.9 respectively. Using the molar extinction coefficient calculated for P257 dye in section 6.3.2.1 $\left(24050 \mathrm{M}^{-1} \mathrm{~cm}^{-1}\right)$ and the surface coverage of this dye on $\mathrm{TiO}_{2}$ surface $\left(3.9 \times 10^{-8}\right.$ moles $/ \mu \mathrm{m}^{3}$, the percentage of P-257 dye desorbed from the working electrode under different conditions of photo-electrochemical growth of PEDOT were calculated and presented in Table 6.6 and Table 6.7. The maximum amount of P-257 dye desorbed from the $\mathrm{TiO}_{2}$ photo-anode after PEP was 37\% (Table 6.7). Images of the electrolytic solutions, as well as the film of the working electrode generated after photo-electrochemical polymerization, are presented in Figures 6.10 and 6.11, respectively. It was observed that using a longer growth period of PEDOT, and applying higher light intensities during photo-electrochemical polymerization, led to more dye desorption from the surface of the working electrode (photo-anode). 


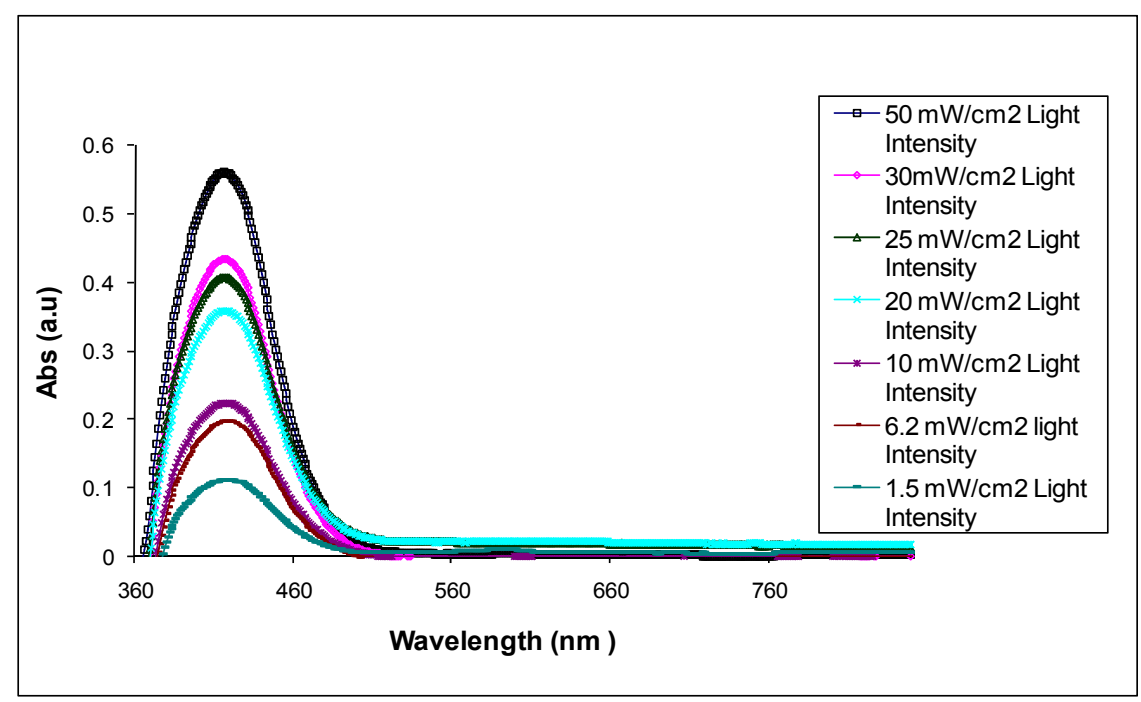

Figure 6.8: UV-Vis spectra of P-257 dye desorption solutions (in electrochemical cell) from photo-anodes when different intensities of light were employed during PEP.

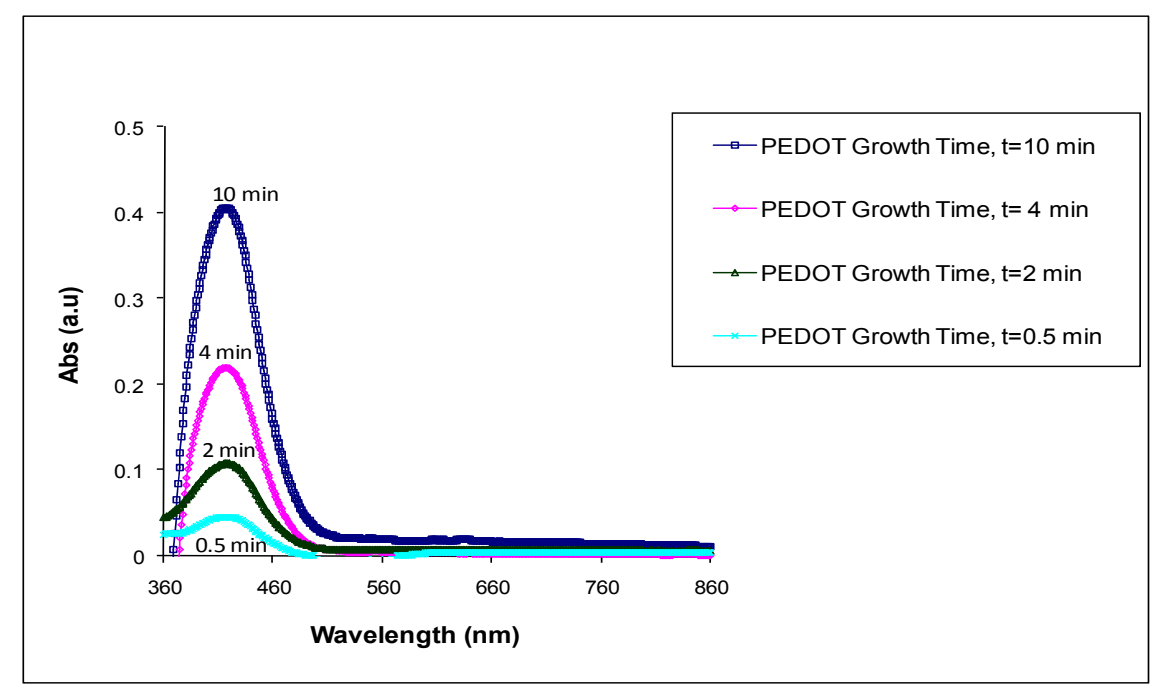

Figure 6.9: UV-Vis spectra of $\mathrm{P}-257$ dye desorption solutions (in electrochemical cell) of from photo-anodes after PEP using various growth times of PEDOT. 


\subsubsection{Calculation of the amount of P-257 dye desorbed from samples prepared} by PEP done under constant $200 \mathrm{mV}$ with $25 \mathrm{~mW} / \mathrm{cm} 2$ light irradiated (anode side) for $10 \mathrm{~min}$.

The absorbance of amount of desorbed dye in the electrolyte:

$\mathrm{A}=0.248$ at $416 \mathrm{~nm}$

$c=\mathrm{A}_{416} / \varepsilon_{416}=0.248 / 24,050=10.3 \times 10^{-6}$ moles $/ \mathrm{L}$.

So, number of moles lost due to desorption, $\mathrm{n}_{\text {lost }}=c \times \mathrm{V}=10.3 \times 10^{-6}$ moles $/ \mathrm{L} \times$ $0.008 \mathrm{~L}$

$$
=8.24 \times 10^{-8} \text { moles. }
$$

But initially it was found that the surface coverage of $\mathrm{P}-257$ dye in $\mathrm{TiO}_{2}$ film

$$
\Gamma=3.9 \times 10^{-8} \text { moles } / \mu \mathrm{m}^{3} \text {. }
$$

Number of moles initially, $\mathrm{n}_{0}=3.9 \times 10^{-8} \times($ area $\times$ thickness $)$ of film

$$
=4.97 \times 10^{-7} \text { moles. }
$$

So $\%$ age desorption of the dye $=\left[\mathrm{n}_{\text {lost }} / \mathrm{n}_{0}\right] \times 100$

$$
\begin{aligned}
& =\left[8.24 \times 10^{-8} / 4.972 \times 10^{-7}\right] \times 100 \\
& =16.6 \% .
\end{aligned}
$$

Similarly for other conditions, the desorbed dye was calculated by the above method.

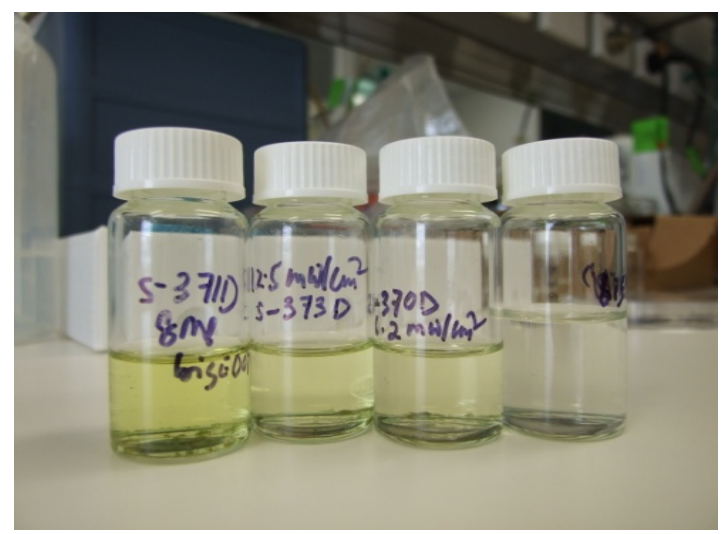

Figure 6.10: Images of solution after photo-electrochemical polymerization of bisEDOT with different light intensities. The white colour of bis-EDOT solution (extreme right bottle) changes yellow colour due to the dye desorption from photo anode. 


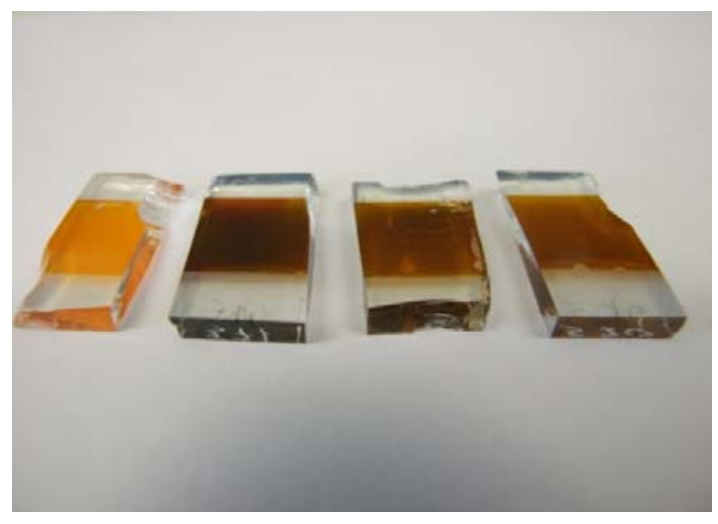

Figure 6.11: Images of the original P-257 dye sensitized $\mathrm{TiO}_{2}$ film ( far left) and of the dye sensitized $\mathrm{TiO}_{2}$ film after PEP of PEDOT using different light intensities $\left(25,12.5,6.2 \mathrm{~mW} / \mathrm{cm}^{2}\right.$, left to right).

\subsubsection{Calculation of total polymerization charge}

The total polymerisation charge when PEP was carried out different light intensities (Figure 6.12) as well as with different growth periods of PEDOT (Figure 6.13) was calculated by the integration of the polymerization currents. When PEP was performed under $20 \mathrm{~mW} / \mathrm{cm}^{2}$ light intensity, the highest polymerization charge was achieved $\left(14.1 \mathrm{mC} / \mathrm{cm}^{2}\right)$. The total polymerization charge increased linearly with an increase in light intensity, up to a $20 \mathrm{~mW} / \mathrm{cm}^{2}$ light intensity, of the photo-anode on which the PEDOT was grown in situ.

Similarly, when the period of growth of PEDOT was varied, it was observed that the total polymerization charge increased during polymer growth and the percentage of P-257 dye loss from the photo-anode also increased. This increase of polymerization charge was attributed to the lower amount of dye that was removed from the photoanode during PEP; which was confirmed by the UV-Vis spectra of the electrolytic solutions in the electrochemical cell (Figure 6.8 and Figure 6.9). The total polymerization charge values decreased with increase of light intensity from 30 to 50 $\mathrm{mW} / \mathrm{cm}^{2}$ (Table 6.4), whereas the polymerization charge increased between 20-30 $\mathrm{mW} / \mathrm{cm}^{2}$ light intensity. This is because of dye loss from the photo-anode during PEP. When the light intensity was lowered below $20 \mathrm{~mW} / \mathrm{cm}^{2}$, the total 
polymerization charge decreased. From Table 6.5, it is observed that the calculated total polymerization charge was $\sim 13 \mathrm{mC} / \mathrm{cm}^{2}$ when PEP was carried out for 10 minutes with $25 \mathrm{~mW} / \mathrm{cm}^{2}$ light intensity.

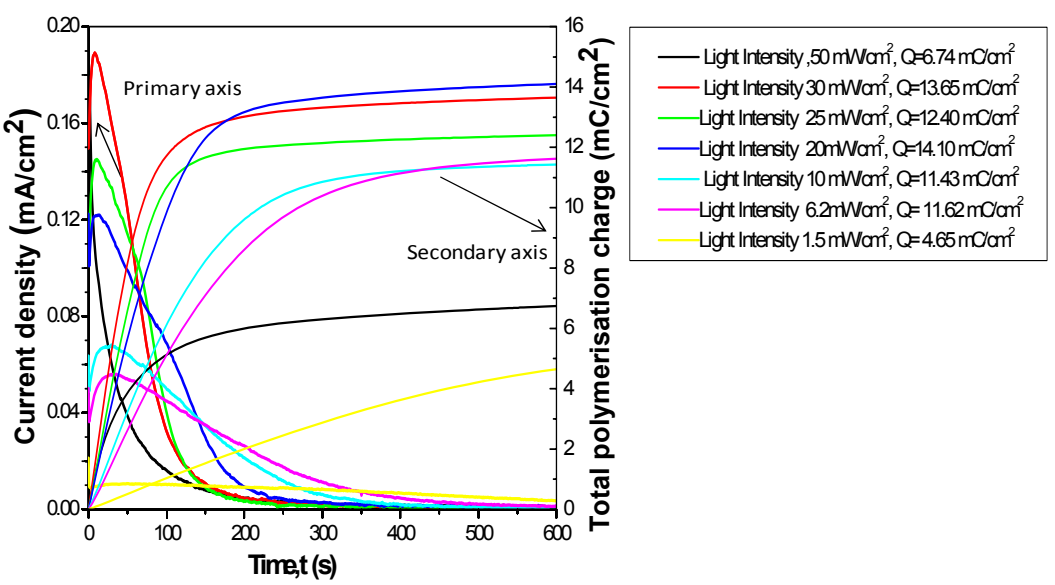

Figure 6.12: The trend of photo-current (primary axis) and the total photopolymerization charge (secondary axis) calculated for P-257 dye system when PEDOT was grown using different light intensities during PEP.

Table 6.4: The summary of the total polymerization charge when using various light intensities during PEP at an applied potential of $200 \mathrm{mV}$.

\begin{tabular}{|c|c|c|c|}
\hline Sample & $\begin{array}{c}\text { Light Intensity } \\
\left(\mathrm{mW} / \mathrm{cm}^{2}\right)\end{array}$ & $\begin{array}{c}\text { Dye } \\
\text { Loss } \\
(\%)\end{array}$ & $\begin{array}{c}\text { Total Polymerization } \\
\text { Charge }\left(\mathrm{mC} / \mathrm{cm}^{2}\right)\end{array}$ \\
\hline S-406D & 50 & 37.3 & 6.74 \\
\hline S-409D & 30 & 31.8 & 13.7 \\
\hline S-407D & 25 & 26.6 & 12.4 \\
\hline S-405D & 10 & 15.1 & 11.4 \\
\hline S-403D & 6.2 & 13.5 & 11.6 \\
\hline S-404D & 1.5 & 8.00 & 4.65 \\
\hline
\end{tabular}




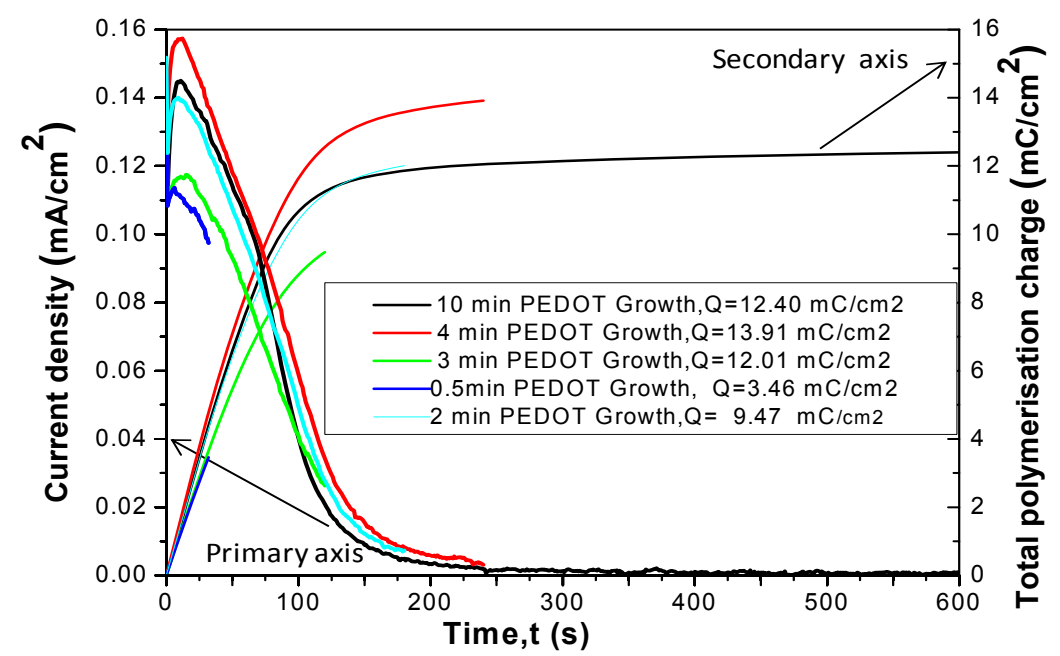

Figure 6.13: The photo-current (primary axis) and the total photo-polymerization charge (secondary axis) calculated for the P-257 dye system when PEDOT was grown for different periods.

Table 6.5: Total polymerization charge when using different periods of PEDOT growth during PEP at $25 \mathrm{~mW} / \mathrm{cm}^{2}$ and an applied potential of $200 \mathrm{mV}$.

\begin{tabular}{|c|c|c|c|c|}
\hline Sample & $\begin{array}{c}\text { Period of } \\
\text { PEDOT }\end{array}$ & $\begin{array}{c}\text { Light } \\
\text { Intensity } \\
\text { Growth } \\
(\mathrm{min})\end{array}$ & $\begin{array}{c}\text { Dye } \\
\text { Loss } \\
\left(\mathrm{mW} / \mathrm{cm}^{2}\right)\end{array}$ & $\begin{array}{c}\text { Total } \\
\text { Polymerization } \\
\text { Charge }\left(\mathrm{mC} / \mathrm{cm}^{2}\right)\end{array}$ \\
\hline S-407D & 10 & 25 & 26.6 & 12.4 \\
\hline S-402 D & 4 & 25 & 15.2 & 13.9 \\
\hline S-411D & 3 & 25 & 19.4 & 12.0 \\
\hline S-412D & 2 & 25 & 8.00 & 9.47 \\
\hline S-415D & 0.5 & 25 & 3.98 & 3.46 \\
\hline
\end{tabular}




\subsubsection{Photovoltaic performance}

\subsubsection{Photovoltaic (PV) performance using Zinc-porphyrin (GD2) and dimer (P-10) dyes}

All ssDSC devices in this chapter were assembled by sandwiching PEDOT /dyed $\mathrm{TiO}_{2}$ (working) electrode and Goretex ${ }^{\circledR}$-Au-PEDOT (counter) electrode together by mechanical clipping.

\subsubsection{Zinc-porphyrin (GD2) dye}

It is observed that device performances such as $V_{\mathrm{oc}}, J_{\mathrm{sc}}$, fill factor and efficiency all increase when the photo-polymerization light intensity employed for PEDOT deposition was increased from 3.2 to $4.2 \mathrm{~mW} / \mathrm{cm}^{2}$, but decreased at higher light intensities (Table 6.6). It reveals from the PV performance data (Table 6.6) that the optimum $\mathrm{TiO}_{2}$ thickness was $5.5 \mu \mathrm{m}$. The efficiency of the device is mainly dependent on the short-circuit current density of the cells and it is observed at low current. The low photo-current may be due to the optical filtering of PEDOT. The pores in $\mathrm{TiO}_{2}$ electrode may not be completely filled by PEDOT. This may be due to the pores of $\mathrm{TiO}_{2}$ channels becoming blocked by PEDOT once photo-electrochemical polymerization starts, so the bis-EDOT can not reach deeper into the $\mathrm{TiO}_{2}$ film.

The incident photon to current efficiency (IPCE) spectrum of a PEDOT ssDSC was obtained to see the sensitization effect of zinc-porphyrin GD2 dye. For the GD2 dye, it was found that the IPCE for the Soret band was $42 \%$ and the IPCE for the Q band was $30 \%$, corresponding to a combined current of $5.04 \mathrm{mAcm}^{-2}$. The high UV-Vis absorption of this zinc-porphyrin dye indicates high light harvesting capability leading to potentially high efficiency devices. From UV-Vis spectra, it is evident that a broad absorption band $\lambda>600 \mathrm{~nm}$ is observed and is a characteristic of p-doped PEDOT. The absorption by PEDOT $\lambda>700 \mathrm{~nm}$ did not contribute any reasonable photo-currents which leads the low PV performance of the ssDSCs. 
The photo-current generated during in situ PEP of bis-EDOT using zinc porphyrin (GD2) dye-coated $\mathrm{TiO}_{2}$ films is shown in Figure 6.14. The photo-polymerization was performed for up to 30 minutes using various intensities of light irradiated on the dyed- $\mathrm{TiO}_{2}$ (working electrode) side. It was found that $4.2 \mathrm{~mW} / \mathrm{cm}^{2}$ light intensity produced the best photo-anodes because this light intensity allowed effective photo polymerization of bis-EDOT to PEDOT.

Table 6.6: Comparison of photovoltaic parameters obtained for solid-state DSCs using different light intensities during PEP of bis-EDOT and different thicknesses of $\mathrm{TiO}_{2}$. Mesured under one sun conditions (AM 1.5), $V_{\text {oc }}$ : open-circuit voltage; $J_{\text {sc: }}$ short-circuit current density; FF: fill factor; $\eta$ : efficiency of device.

\begin{tabular}{|c|c|c|c|c|}
\hline $\begin{array}{c}\text { Light } \\
\text { Intensities } \\
\left(\mathrm{mW} / \mathrm{cm}^{2}\right)\end{array}$ & $V_{\mathrm{oc}}(\mathrm{mV})$ & $J_{\mathrm{sc}}\left(\mathrm{mA} / \mathrm{cm}^{2}\right)$ & $\mathrm{FF}$ & $\eta(\%)$ \\
\hline 3.2 & 555 & 2.78 & 0.66 & 1.02 \\
\hline 4.2 & 650 & 5.04 & 0.63 & 2.06 \\
\hline 5.2 & 525 & 4.71 & 0.55 & 1.35 \\
\hline 7.2 & 505 & 2.87 & 0.58 & 0.84 \\
\hline \begin{tabular}{|c|c|c|c|c|c|}
\hline $\mathrm{TiO}$ \\
$\mathrm{Thickness}$
\end{tabular} & $\begin{array}{c}V_{\mathrm{OC}} \\
(\mathrm{mV})\end{array}$ & $\begin{array}{l}J_{\mathrm{SC}} \\
\left(\mathrm{mA} / \mathrm{cm}^{2}\right)\end{array}$ & $\mathrm{FF}$ & $\eta(\%)$ \\
\hline \begin{tabular}{|c|c|c|c|c|}
\hline$\mu \mathrm{m})$ \\
\hline 3
\end{tabular} & 535 & 4.65 & 0.45 & 1.11 \\
\hline 5.5 & 650 & 5.04 & 0.45 & 2.06 \\
\hline 7.5 & 565 & 0.67 & 0.54 & 0.20 \\
\hline
\end{tabular}




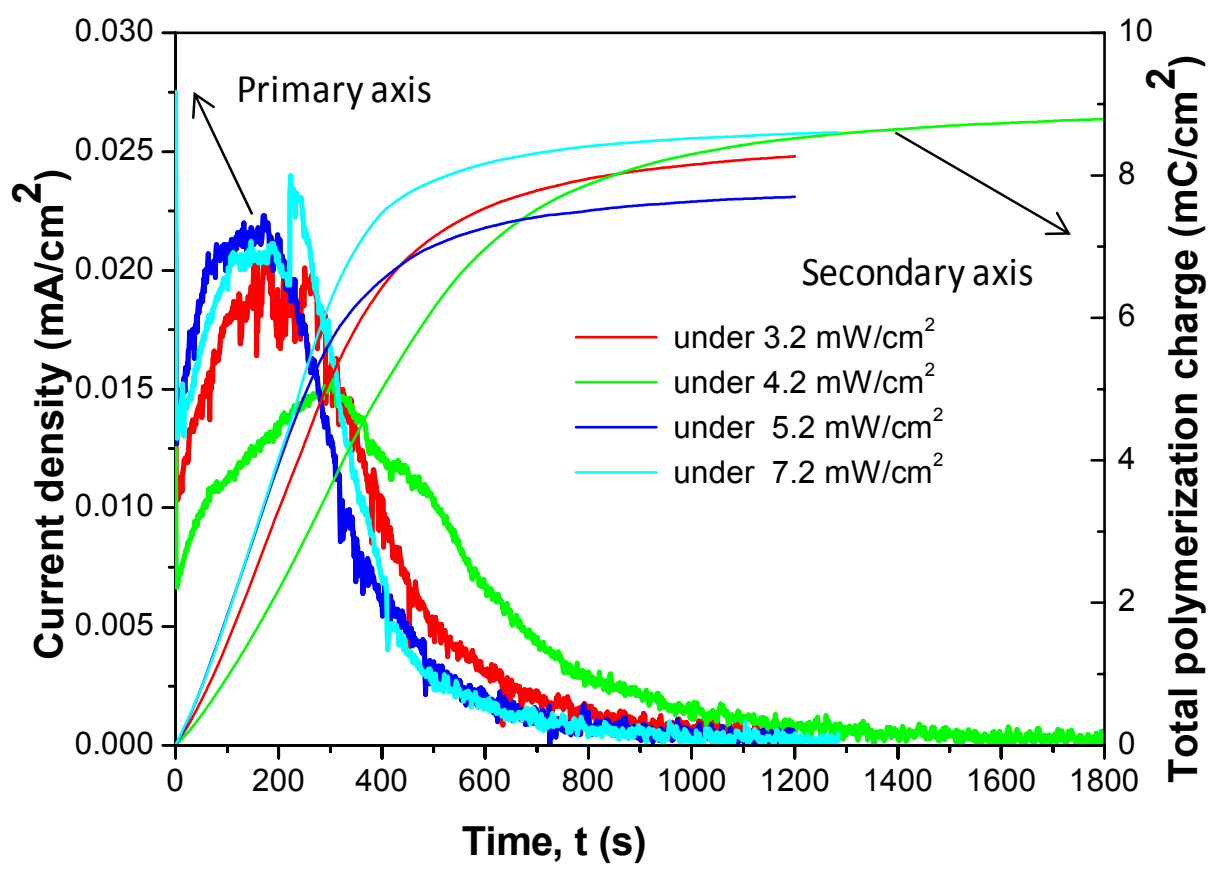

Figure 6.14: The photo-current (primary axis) and the total photo-polymerization charge (secondary axis) calculated for GD2 dye system.

The total polymerisation charge was found to vary from $5-20 \mathrm{mC} / \mathrm{cm}^{2}$ depending upon the growth condition of PEDOT during photo-polymerization, such as wavelength, thickness of $\mathrm{TiO}_{2}$ film, and intensity of light with different light sources. The maximum polymerization charge was approximately $9 \mathrm{mC} / \mathrm{cm}^{2}$.

Figure 6.14 illustrates that while the photocurrent increased initially, it then decreased after 3-5 minutes of photo-polymerization. It appears that Zn-porphyrin dye excitation is crucial to the in situ photo-electrochemical polymerization process. Dye excited by illumination oxidises bis-EDOT and PEDOT is formed. Gradually the PEDOT covers the mesoporous surface of the working electrode, shading the GD2 dye and thereby weakening the photo-electrochemical driving force for bisEDOT polymerization. The process continues, ever more slowly, ideally until the GD2 dye is fully covered with PEDOT. At this stage, PEDOT probably partially blocks the light reaching the dye. In practice, polymerized PEDOT may either prevent bis-EDOT from reaching the FTO side of the dyed titania or shades the dye 
on the FTO side. To improve fill factor and short-circuit current, a better polymerization method is required to ensure good electronic contact with the entire depth of the dyed titania.

It is observed from Figure 6.15 that device performances such as $V_{\mathrm{oc}}, J_{\mathrm{sc}}$, fill factor and efficiency all increased when the photo-polymerization light intensity increased from 3.2 to $4.2 \mathrm{~mW} / \mathrm{cm}^{2}$, but decreased at higher intensities. The IPCE for a cell with a Goretex ${ }^{\circledR}-P E D O T$ counter electrode, is also shown as an inset in Figure 6.15. The IPCE shows the effect of the broad absorption band above $600 \mathrm{~nm}$ of Z907 dye. The wavelengths above $700 \mathrm{~nm}$ do not contribute significantly to the photocurrent. The peak at $570 \mathrm{~nm}$ is characteristic of the GD2 dye. This response is typical of the working electrodes utilised throughout the present study and, as expected, the different counter-electrodes did not impact significantly upon the measured IPCE.

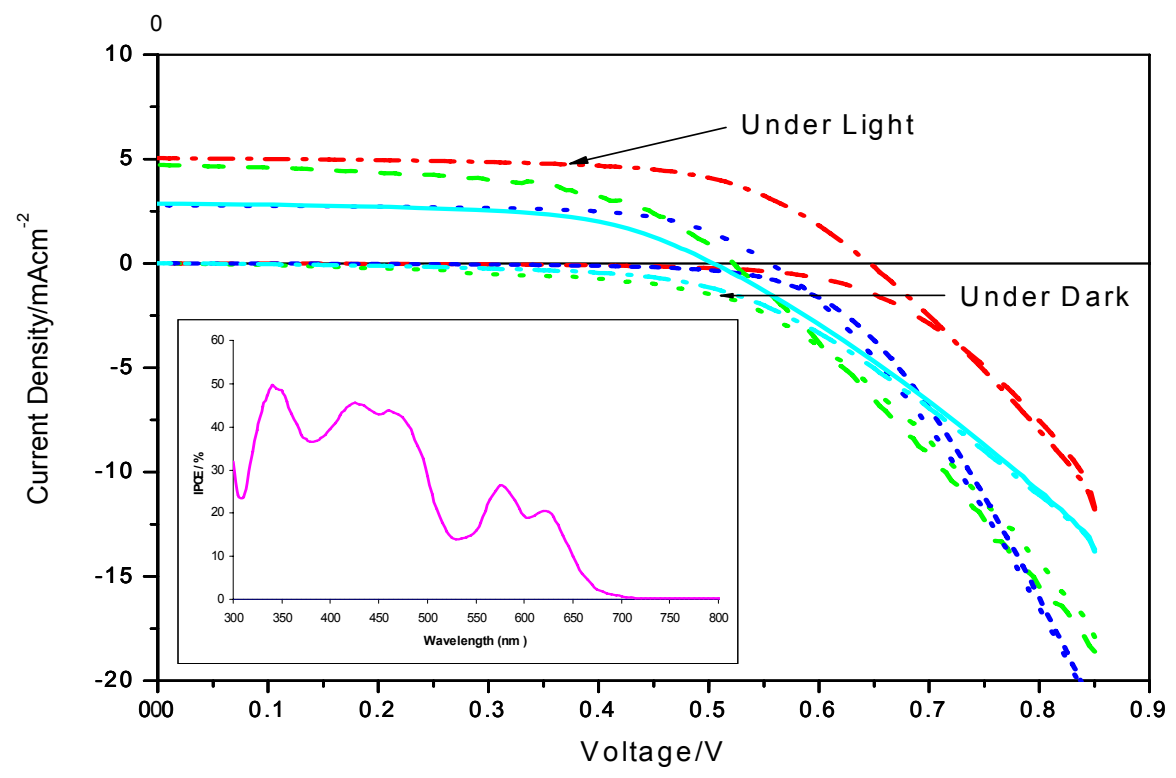

Figure 6.15: The current-voltage curves of four solid-state DSC using GD2 dye and employing different intensities of light irradiated during PEP of bisEDOT for working electrode preparation. Inset shows the IPCE for the best device.

Z907 dye (ruthenium based) and zinc porphyrin dye sensitized photo-anodes with PEDOT, have been used to study influence of morphology on device performance. The influence of film morphology on the device performance of the photo- 
electrochemically polymerized PEDOT film has been elucidated using Atomic Force Microscopy AFM (Figures 6.16)

It was observed that in the case of the zinc-porphyrin GD2 dye, the ssDSC had an efficiency of $2 \%$. The AFM image of photo-anode (Figure 6.16), observed that the size of the PEDOT particles was found to be very uniform and smaller $(\sim 10 \mathrm{~nm})$ than that measured for the corresponding Z907 sensitized (discussed in Chapter 4) device (where PEDOT sizes were found to be $\sim 40 \mathrm{~nm}$ ). AFM images of each layer reveal nano-scale detail within each stratum, including the compact layer $\left(\mathrm{TiO}_{2}\right)$, which allows the study of the pores between particles, and the nonporous electrode, revealing the smoothness of the film. Electrodes were studied both before and after deposition of PEDOT on the dyed $\mathrm{TiO}_{2}$, allowing the correlation of these morphological measurements with the photovoltaic performance of each device.

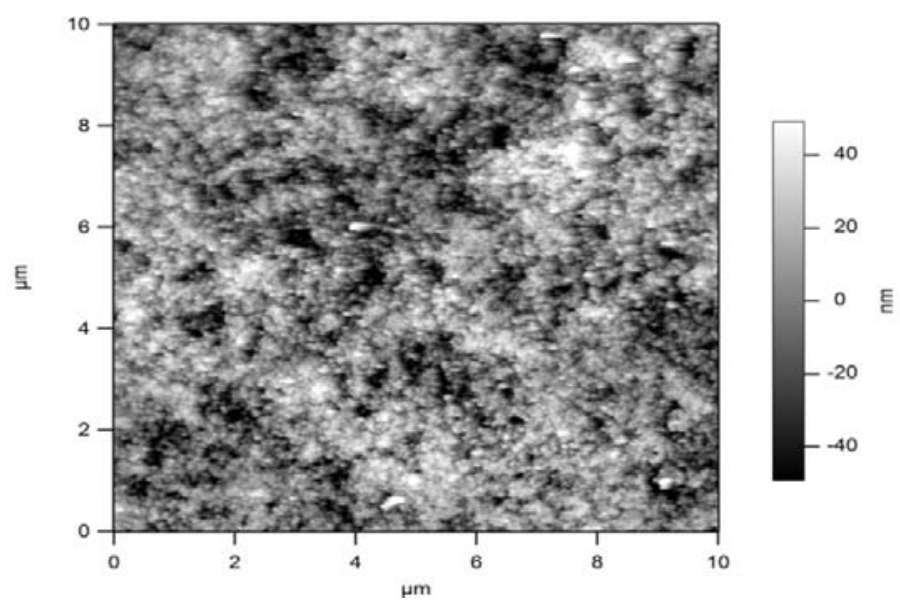

Figure 6.16: AFM height image of PEDOT/GD2 dyed $\mathrm{TiO}_{2}$ photo-anode.

ssDSCs using both iodine-free PEDOT hole transport material and ruthenium-free efficient zinc-porphyrin sensitizer GD2 dye were made for the first time. The PEDOT was photo-electrochemically polymerized from bis-EDOT in situ using the zinc-porphyrin dye instead of the high-cost ruthenium-based hydrophobic Z907 dye. The best device performance obtained in simulated AM1.5 (100 mW cm$\left.{ }^{-2}\right)$ illumination was $J_{\mathrm{sc}}$ of $5.04 \mathrm{~mA} / \mathrm{cm}^{2}, V_{\text {oc }}$ of $650 \mathrm{mV}$, and photoelectric conversion 
efficiency of $2.06 \%$. These efficiency results are $33 \%$ less efficient than those found using the Z907 dye.

\subsubsection{Dimer (P-10) dye}

IPCE and JV results for the best ssDSCs fabricated here using the P-10 zincporphyrin dimer are shown in Figure 6.17 and Figure 6.18 respectively. The dimer dye was utilised because of its high molar extinction coefficient, so a thinner $\mathrm{TiO}_{2}$ film can be used, providing device performance similar to the GD2 dye with a thicker $\mathrm{TiO}_{2}$ layer. Platinum and PEDOT-based counter electrodes were examined separately for comparison. It is believed that further optimisation of photopolymerization conditions should lead to higher efficiency devices.

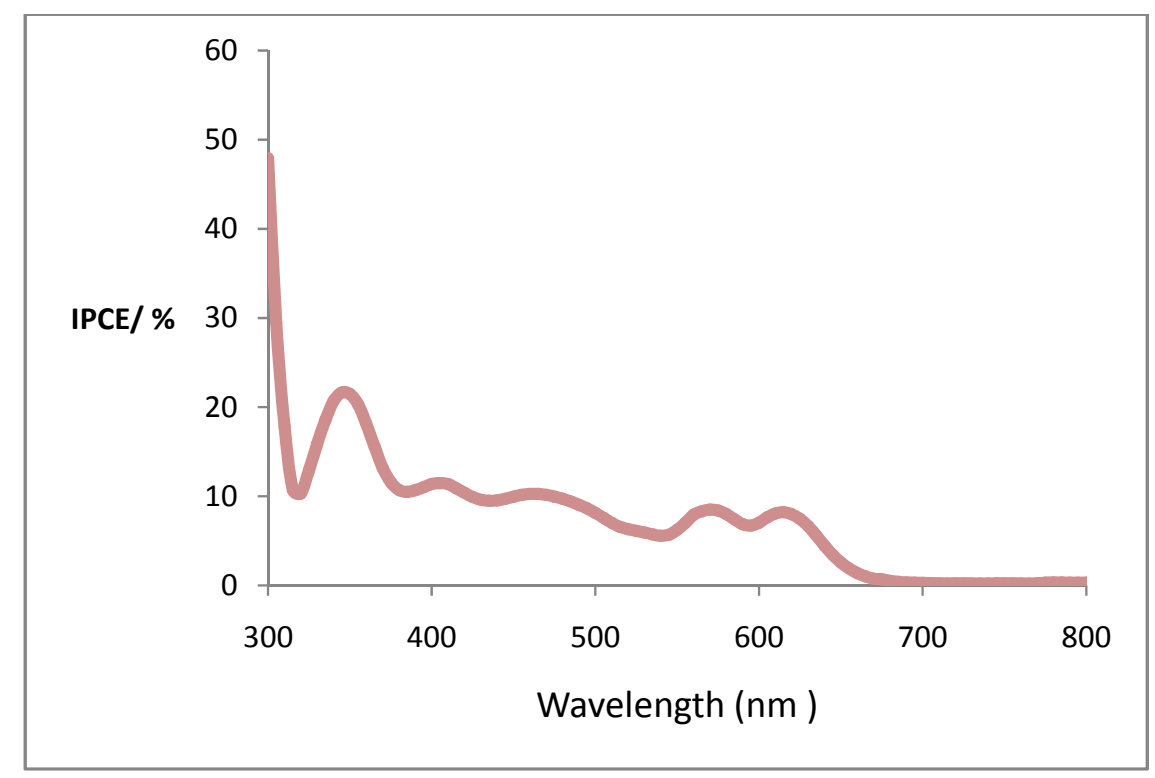

Figure 6.17: IPCE spectrum of an ssDSC employing zinc-porphyrin P-10 dimer dye when Pt is used as counter electrode. 


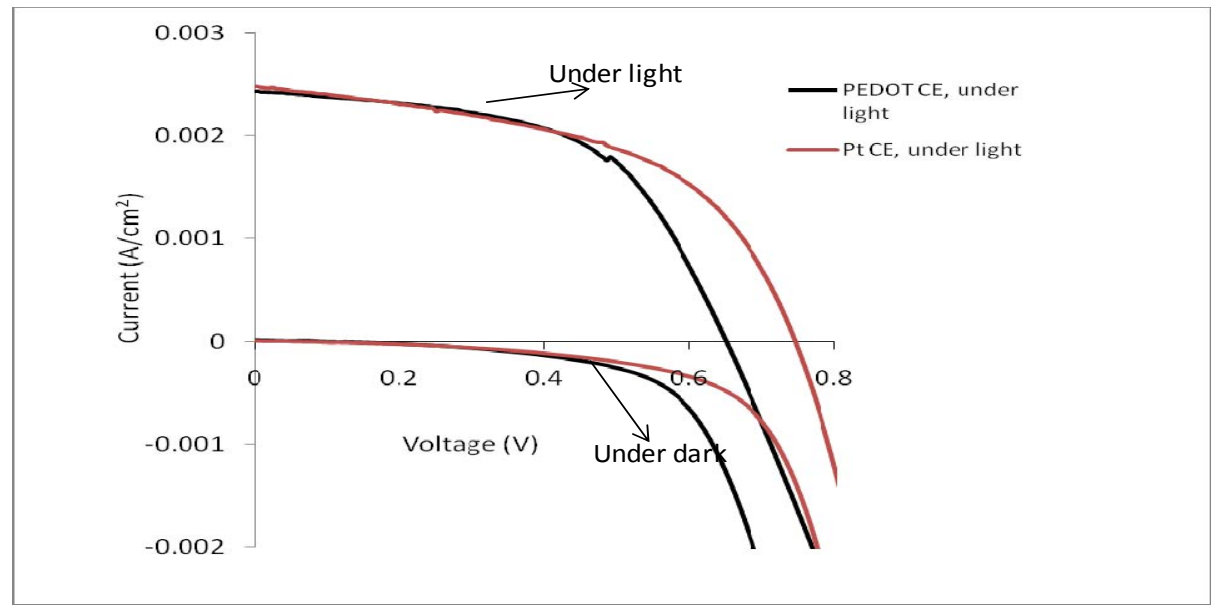

Figure 6.18: Current-voltage curves with different counter electrodes (PEDOT/FTO and Pt/ITO) for ssDSC using P-10 dimer zinc-porphyrin used as photosensitizer.

It is seen from Figure 6.18 that the best device performance obtained in simulated AM1.5 $\left(100 \mathrm{~mW} \mathrm{~cm}^{-2}\right)$ illumination was $J_{\mathrm{sc}}$ of $2.5 \mathrm{~mA} / \mathrm{cm}^{2}, V_{\text {oc }}$ of $750 \mathrm{mV}$, and photoelectric conversion efficiency of $1.06 \%$.

\subsubsection{P-257 sensitizer}

Similarly current-voltage (JV) curves (Figure 6.19) were measured for ssDSCs using $\mathrm{P}-257$ as the sensitizer. These devices were illuminated at $100 \mathrm{~mW} / \mathrm{cm}^{2}$ (AM 1.5) under the same conditions as for the above zinc-porphyrin based devices. As far as the PV performances are concerned, it was observed that when more P-257 dye had desorbed from the photo-anode, the device was less efficient (Tables 6.7 and 6.8). It was also found that a polymerization charge employed in PEDOT deposition produced a better PV performance of the respective devices (Tables 6.7 and 6.8. The photo-electrochemical polymerization was also performed under different conditions, such as different light intensity, different potential, different solvent, and different period of growth of PEDOT. Longer duration of polymerization times is, however, of marginal utility as the current eventually becomes very low. 
From Table 6.7, the photovoltaic performance increases with respect to the intensity of light during photo-polymerisation (starting from 2.5 to $25 \mathrm{~mW} / \mathrm{cm}^{2}$ ). However, with light intensities $>25 \mathrm{~mW} / \mathrm{cm}^{2}$, the PV performances are seen to decrease. Similar trends were also observed when different potentials were applied during PEP, with a linear relationship between potential and efficiency up to $200 \mathrm{mV}$, but thereafter the efficiency decreased with increasing applied potential. The total polymerization charge is initially seen in Table 6.7 to increase with the increasing loss of dye during photo-electrochemical polymerization. The JV graph of the best device is shown in Figure 6.19. Table 6.8 shows the trends of the PV parameters as a function of PEDOT growth time. It can be observed that when the amount of PEDOT growth was increased, the PV performance also increased. The light intensities during PEP also influenced the device performances as shown in Table 6.7. The dye losses in each condition of PEP, on how the working electrode affected the PV performances, are presented in Tables 6.7 and 6.8 .

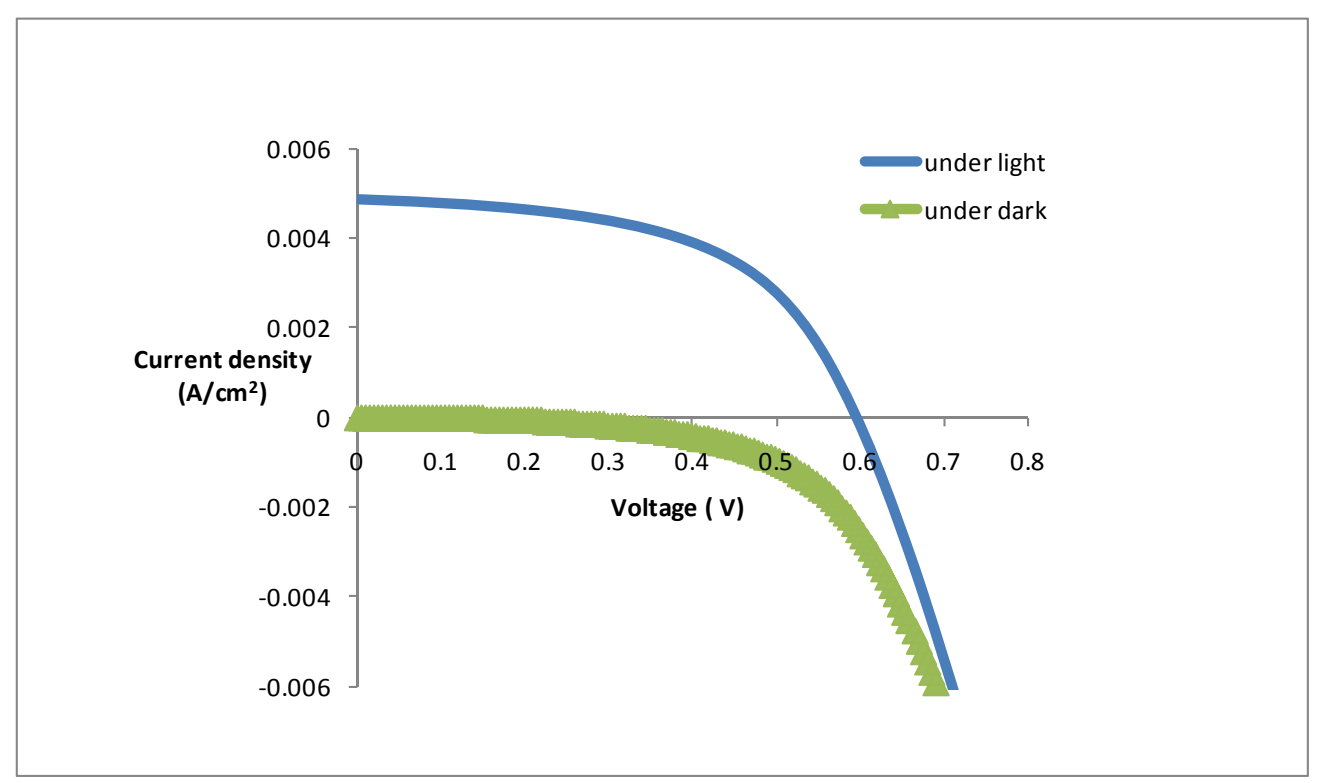

Figure 6.19: The JV curve for the best PEDOT based solid-state dye-sensitized solar cells with Goretex ${ }^{\circledR}$-Au-PEDOT as counter electrode. 
Table 6.7: Summary of performance of P-257 dye sensitized solid-state solar cells in which different irradiation light intensities were used during the growth of PEDOT on photo-anodes.

\begin{tabular}{|c|c|c|c|c|c|c|c|}
\hline Sample & $\begin{array}{c}\text { Light } \\
\text { Intensity } \\
\left(\mathrm{mW} / \mathrm{cm}^{2}\right)\end{array}$ & $\begin{array}{c}\text { Dye } \\
\text { Loss } \\
(\%)\end{array}$ & $\begin{array}{c}\text { Total } \\
\text { Polymerization } \\
\text { Charge } \\
\left(\mathrm{mC} / \mathrm{cm}^{2}\right)\end{array}$ & $\begin{array}{c}V_{\mathrm{oc}} \\
(\mathrm{mV})\end{array}$ & $\begin{array}{c}J_{\mathrm{sc}} \\
\left(\mathrm{mA} / \mathrm{cm}^{2}\right)\end{array}$ & $\begin{array}{c}\text { FF } \\
(\%)\end{array}$ \\
\hline S-406 & 50 & 37.3 & 6.74 & 340 & 3.31 & 0.37 & 0.42 \\
\hline S-409 & 30 & 31.8 & 13.7 & 565 & 4.85 & 0.47 & 1.27 \\
\hline S-407 & 25 & 26.6 & 12.4 & 600 & 4.85 & 0.54 & 1.57 \\
\hline S-408 & 20 & 21.7 & 14.1 & 555 & 4.37 & 0.48 & 1.16 \\
\hline S-405 & 10 & 15.1 & 11.4 & 580 & 3.93 & 0.42 & 0.96 \\
\hline S-403 & 6.2 & 13.5 & 11.6 & 630 & 3.12 & 0.58 & 1.14 \\
\hline S-404 & 1.5 & 8.00 & 4.65 & 515 & 0.79 & 0.53 & 0.21 \\
\hline
\end{tabular}

Table 6.8: Summary of performance of P-257 dye-sensitized solid-state solar cells in which different time periods were used for PEDOT growth.

\begin{tabular}{|c|c|c|c|c|c|c|c|}
\hline $\begin{array}{c}\text { Time of } \\
\text { PEDOT } \\
\text { Growth } \\
\text { (min ) }\end{array}$ & $\begin{array}{c}\text { Light } \\
\text { Intensity } \\
\left(\mathrm{mW} / \mathrm{cm}^{2}\right)\end{array}$ & $\begin{array}{l}\text { Dye } \\
\text { Loss } \\
(\%)\end{array}$ & $\begin{array}{c}\text { Total } \\
\text { Polymerization } \\
\text { charge } \\
\left(\mathrm{mC} / \mathrm{cm}^{2}\right)\end{array}$ & $\begin{array}{c}V_{\mathrm{oc}} \\
(\mathrm{mV})\end{array}$ & $\begin{array}{c}J_{\mathrm{sc}} \\
\left(\mathrm{mA} / \mathrm{cm}^{2}\right)\end{array}$ & $\mathrm{FF}$ & $\begin{array}{c}\eta \\
(\%)\end{array}$ \\
\hline 10 & 25 & 26.6 & 12.4 & 600 & 4.85 & 0.54 & 1.57 \\
\hline 4 & 25 & 15.2 & 13.9 & 595 & 3.65 & 058 & 1.27 \\
\hline 3 & 25 & 19.4 & 12.0 & 580 & 3.09 & 0.51 & 0.91 \\
\hline 2 & 25 & 8.00 & 9.47 & 585 & 2.68 & 0.41 & 0.65 \\
\hline 0.5 & 25 & 3.98 & 3.46 & 510 & 0.49 & 0.36 & 0.09 \\
\hline
\end{tabular}


For comparison, four DSCs were also prepared and their JV curves and IPCE values measured (Figures 6.20 and 6.21). The four devices gave the same sensitization effect; as can be concluded from the IPCE spectra, though the PV parameters reveal minor variations due to the impregnation of electrolyte into the nanomatrix of dyed $\mathrm{TiO}_{2}$. In the liquid DSC, it is observed that the higher the $J_{\mathrm{sc}}$, the higher the $V_{\text {oc }}$ as well as the FF, leading to an efficiency $1 \%$ higher than its counterpart solid-state DSC. The reproducibility of the performance of the four fabricated devices is shown in Table 6.9.

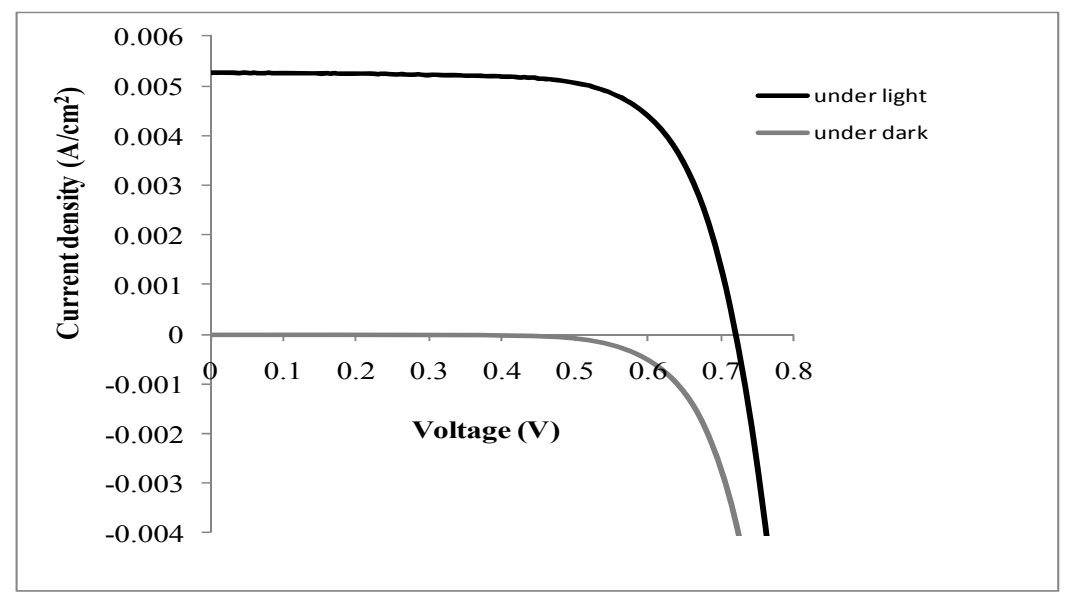

Figure 6.20: The JV curve for the best liquid dye-sensitized solar cell fabricated using P-257 dye as sensitizer.

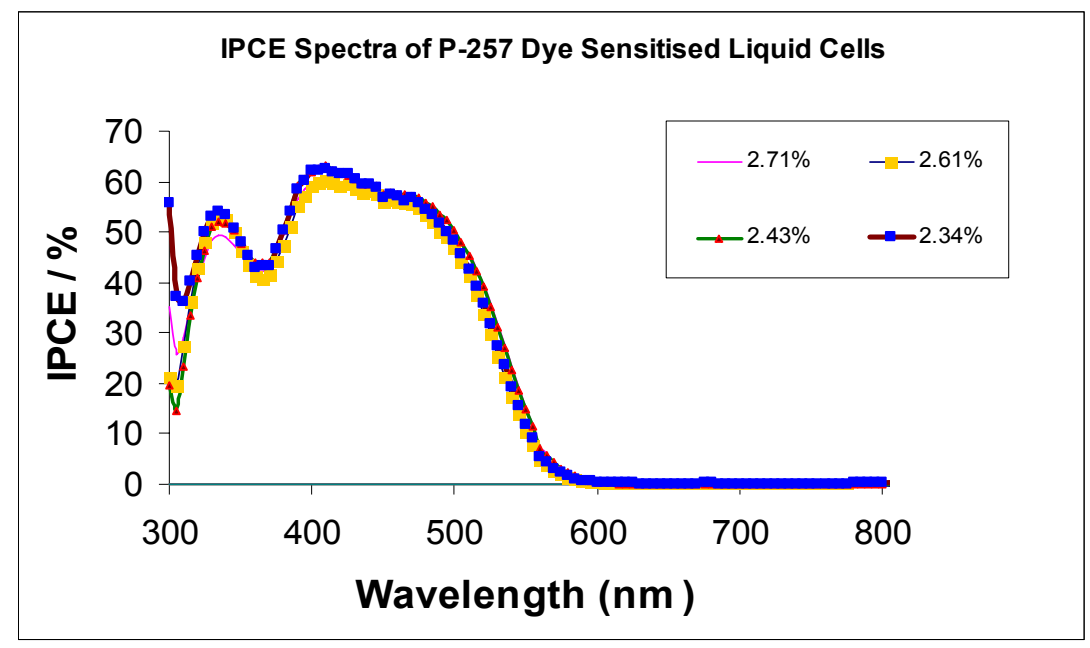

Figure 6.21: The IPCE spectra of four liquid dye-sensitized solar cells fabricated using with P-257 dye. 
Table 6.9: Reproducibility of performance for a P-257 dye sensitized liquid DSCs.

\begin{tabular}{|c|c|c|c|c|}
\hline Sample & $\begin{array}{c}V_{\mathrm{oc}}(\mathrm{mV}) \\
( \pm 4.8 \mathrm{mV}\end{array}$ & $J_{\mathrm{sc}}\left( \pm 0.3 \mathrm{mAcm}^{-2}\right)$ & $\mathrm{FF}( \pm 0.005)$ & $\eta( \pm 0.16 \%)$ \\
\hline S-416D & 720 & 5.32 & 0.71 & 2.71 \\
\hline S-419D & 710 & 5.24 & 0.70 & 2.61 \\
\hline S-418D & 720 & 4.8 & 0.70 & 2.43 \\
\hline S-417 D & 715 & 4.68 & 0.70 & 2.34 \\
\hline
\end{tabular}

N.B: Error values are the average of at least 4 samples. $20 \mathrm{hrs}$ sensitization was performed on $5.5 \mu \mathrm{m}$ thickness of $\mathrm{TiO}_{2}$ film. Testing conditions: one sun ( $\left.\mathrm{AM} 1.5, \mathrm{P}_{\mathrm{in}} 100 \mathrm{mWcm}^{-2}\right)$.

\subsubsection{D-149 dye sensitizer}

During the in situ photo-electrochemical polymerization, some of the dye was observed to desorb from the working electrode. The higher the intensity of light irradiated on the working electrode, the more dye that was released; possibly due to the binding mode of dye or over-oxidation of dye on the working electrode during PEP. The polymerization charge mostly goes to polymerization of the EDOT monomer, but a small fraction comes from diffusion of ionic species as well as from doping of anions into PEDOT.

The over-all performances of such devices when photo-anodes were prepared from bis-EDOT with different intensities of light with methoxypropionitrile solution during PEP, are presented in Table 6.10.

The highest measured total polymerization charge of PEDOT was in the range 13$20 \mathrm{mC} / \mathrm{cm}^{2}$, achieved by potentiostatic polymerization for 30 minutes. Longer duration polymerizations were of marginal utility, as the current became very low. It is observed during PEP that the high intensity $\left(25 \mathrm{~mW} / \mathrm{cm}^{2}\right)$ led to a total charge of $19.5 \mathrm{mC} / \mathrm{cm}^{2}$. The photovoltaic performance parameters for ssDSCs employing D-149 as sensitizer varied with respect to intensity of light during 
photo-polymerization (starting from 2.5 to $25 \mathrm{~mW} / \mathrm{cm}^{2}$ ). However, with higher light intensities the PV performances decreased.

In Table 6.11, all photo-anodes were prepared using bis-EDOT solution in acetonitrile and varying the light intensities during PEP from 6 to $100 \mathrm{~mW} / \mathrm{cm}^{2}$. It was observed that $25 \mathrm{~mW} / \mathrm{cm}^{2}$ light intensity gave the better PV results for D-149 dye (Table 6.11). It is observed (Table 6.11) that when the light intensity was increased during PEP, the total polymerization charge increased as also did overall PV performances.

Table 6.10: Performance of ssDSCs employing with D-149 dye (indoline dye) 24 hrs sensitization with $\mathrm{TiO}_{2}(\sim 5.5 \mu \mathrm{m})$.

\begin{tabular}{|c|c|c|c|c|c|}
\hline $\begin{array}{c}\text { bis-EDOT } \\
\text { solution }\end{array}$ & $\begin{array}{c}\text { Light intensity during } \\
\text { PEP }\left(\mathrm{mW} / \mathrm{cm}^{2}\right)\end{array}$ & $\begin{array}{c}V_{\mathrm{oc}} \\
(\mathrm{mV})\end{array}$ & $\begin{array}{c}J_{\mathrm{sc}} \\
\left(\mathrm{mA} / \mathrm{cm}^{2}\right)\end{array}$ & $\begin{array}{c}\mathrm{FF} \\
\text { Efficiency } \\
(\%)\end{array}$ \\
\hline *MPN & 22.5 & 480 & 2.59 & 0.39 & 0.48 \\
\hline MPN & 25 & 560 & 2.99 & 0.46 & 0.77 \\
\hline $\mathrm{MPN}$ & 5 & 485 & 2.37 & 0.31 & 0.31 \\
\hline
\end{tabular}

*MPN: Methoxypropionitrile.

With ACN and MPN, some dye desorbed out from the working electrode during photo-electrochemical polymerization, which lowered the PV performance of the ssDSCs. Acetonitrile (ACN) was used a solvent for bis-EDOT solution which has been already mentioned throughout the thesis. This is the most common solvent used for photo-electrochemical polymerization because it is an aprotic polar solvent and having a moderate dielectric constant $(\varepsilon=36)$ with high dipole moment 3.92D. This is good solvent which dissolves supporting electrolytes such as $\mathrm{LiClO}_{4}, \mathrm{TBAPF}_{6}$ and 
many more. (The dye detachment may be due to the acidic affect of $\mathrm{TiO}_{2}$. This dye is not stable under photo-electrochemical conditions on surface of $\mathrm{TiO}_{2}$. It has been reported earlier that generally the problem with organic dyes is their stability as well the tendency to produce reactive radicals after electron injection.

It has been calculated the surface coverage of this P-257 dye is higher than the Ru and porphyrin dye which indicates the possible reason of dye aggregation i.e. dye molecule aggregating with other dye molecule instead of anchoring/grafting to the $\mathrm{TiO}_{2}$ surface with the help of $-\mathrm{COOH}$ group. The anchoring ligands which are present in the dye molecule also influence the electron injection efficiency of the dye.

3- Methoxypropionitrile an alternative solvent instead of acetonitrile was used during photo-electrochemical polymerization because of its relatively high vapor pressure which allows non-enclosed experiments to be carried out with a not too high rate of evaporation. But in both solvents the dyes desorption could not stop.

The alternative to these two solvents will be another aprotic polar solvent Dichloromethane (DCM), having low dielectric constant $(\varepsilon=9)$ with low dipole moment (1.6D) that stabilizes the radical cations. The radical cations are very much prone to nucleophillic attack. DCM solvent which contains donor electron pairs is good nucleophile. The solvent which is Lewis bases destabilize radical cations (e.g. $\mathrm{ACN}$ ) where as DCM has negligible Lewis base properties. The photoelectrochemical polymerization could be enhanced without loosing any dye from $\mathrm{TiO}_{2}$ surface and is a matter of investigation. The oxidation / reduction potential of intended organic dye molecule to radical formation depend upon the nature of solvent. 
Table 6.11 : Summary of performances of ssDSCs using D-149 indoline dye as sensitizer.

\begin{tabular}{|c|c|c|c|c|}
\hline Light intensity during PEP $\left(\mathrm{mW} / \mathrm{cm}^{2}\right)$ & $\begin{array}{c}V_{\mathrm{oc}} \\
(\mathrm{mV})\end{array}$ & $\begin{array}{c}J_{\mathrm{sc}} \\
\left(\mathrm{mA} / \mathrm{cm}^{2}\right)\end{array}$ & $\mathrm{FF}$ & $\begin{array}{c}\text { Efficiency } \\
(\%)\end{array}$ \\
\hline 200 & 180 & 0.47 & 0.31 & 0.03 \\
\hline 100 & 355 & 1.45 & 0.39 & 0.20 \\
\hline 30 & 535 & 3.34 & 0.39 & 0.71 \\
\hline 25 & 300 & 2.84 & 0.32 & 0.24 \\
\hline 25 & 530 & 2.61 & 0.49 & 0.67 \\
\hline 25 & 535 & 3.26 & 0.46 & 0.72 \\
\hline 23 & 390 & 2.02 & 0.38 & 0.30 \\
\hline 20 & 355 & 2.84 & 0.30 & 0.31 \\
\hline 6 & 285 & 1.00 & 0.34 & 0.10 \\
\hline
\end{tabular}




\section{4 Conclusions}

Solid-state DSCs fabricated utilising a zinc-porphyrin-based dye (GD2) and photopolymerized PEDOT as HTM are found to have comparable performances to related ruthenium based dye (Z907) based solid-state DSCs, with relatively high open circuit voltages. The GD2 dye based ssDSC exhibits high photocurrent density because of its flat IPCE spectrum over the entire visible spectral region. Although the GD2 dye based device displays a further broad IPCE spectrum extending to the near-IR region, cell performance is not improved because of smaller absorption in the IR, and IR light absorption by PEDOT. The GD2 dye therefore shows significant potential as a cost-effective alternative to the current Ru-based dye Z907 as a photo-sensitizer for solid-state DSCs. Future investigations should further optimise cell fabrication to increase efficiency and to better understand the mechanism of operation.

Experimental observations with the P-257 dye indicates that it's desorption from the photo-anode is due a combination of the effects of light intensity, applied potential and solvent during the PEP. The best P-257 dye-sensitized solid-state solar cell is made with Goretex ${ }^{\circledR}$-Au-PEDOT as counter electrode using $25 \mathrm{~mW} / \mathrm{cm}^{2}$ light intensity during the PEP deposition of PEDOT on the dyed $\mathrm{TiO}_{2}$. A liquid DSC fabricated using the same $5.5 \mu \mathrm{m} \quad \mathrm{TiO}_{2}$ film (without adding co-adsorbent chendeoxycholic acid into the dye solution) and Pt/ ITO as counter electrode is found to have an efficiency of $2.71 \%$, which is a little higher than that of the solid-state DSC (1.57\%). This shows that the P-257 dye is a metal-free dye which can be used as a potential photo-sensitizer for both kinds of devices.

In summary, it is demonstrated that the indoline dye D-149 can be used as an efficient photo-sensitizer for solid-state DSCs. These solar cells show a comparable photovoltaic performance (nearly $1 \%$ efficient) to ssDSCs using zinc-porphyrin dimer (P-10) as dye and similar intensity of light during photo-polymerization deposition of PEDOT. The low efficiency of these ssDSCs with these dyes is because of dye desorption from the photoanode during photolectrochemical polymerization. 


\section{5 References}

1. Chiba, Y., Islam, A., Watanabe, Y., Komiya, R., Koide, N., and Han, L., Japanese Journal of Applied Physics, 45 (2006) 638-640.

2. Wang, Q., Ito, S., Grätzel, M., Fabregat-Santiago, F., Mora-Seró, I., Bisquert, J., Bessho, T., and Imai, H., Journal of Physical Chemistry B, 110 (2006) 2521025221.

3. Grätzel, M., Inorganic Chemistry, 44 (2005) 6841-6851.

4. Horiuchi, T., Miura, H., Sumioka, K., and Uchida, S., Journal of the American Chemical Society, 126 (2004) 12218-12219.

5. Wang, M., Xu, M., Shi, D., Li, R., Gao, F., Zhang, G., Yi, Z., Humphry-Baker, R., Wang, P., Zakeeruddin, S.M., and Gratzel, M., Advanced Materials, 20 (2008) 4460-4463.

6. Yum, J.H., Chen, P., Gratzel, M., and Nazeeruddin, M.K., Chem Sus Chem, 1 (2008) 699-707.

8. Fukuri, N., Saito, Y., Kubo, W., Senadeera, G.K.R., Kitamura, T.,Wada, Y., andYanagida, S., Journal of The Electrochemical Society, 151 (2004) 17451748 .

9. Yanagida, S., Yu, Y., and Manseki K., Accounts of Chemical Research, 42 (2009) 1827-1838.

10. Xia, J., Masaki, N., Lira-Cantu, M., Kim, Y., Jiang K., and Yanagida, S., Journal of the American Chemical Society, 130 (2008) 1258-1263.

11. Mozer, A. J., Panda, D. K., Gambhir, S., Winther-Jensen, B., and Wallace, G.G., Journal of the American Chemical Society, 132 (2010) 9543-9545.

12. Yanagida, S., Yu, Y., and Manseki K., Accounts of Chemical Research, 42 (2009) 1827-1838.

13. Burrell, A.K., Officer, D.L., Plieger, P.G., and Reid, D.C.W., Chemical Reviews, 101( 2001) 2751-2796.

14. Mozer, A.J., Wagner, P., Officer, D.L., Wallace, G. G., Campbell, W.M., Miyashita, M.,, Sunahara, K.,and Mori, S., Chemical. Communications, 39 (2008) 4741-4742. 
15. Mozer, A.J., Griffith, M.J., Tsekouras, G., Wagner, P., Wallace, G. G., Mori, S., Sunahara, K., Miyashita, M., Earles, J. C., Gordon, K.C., Du, L., Katoh, R., Furube, A. and Officer, D.L., Journal of the American Chemical Society, 131 (2009) 15621-15623.

16. Bonfantini, E.E., Burrell, A.K., Officer, D.L., and Reid, D.C.W., McDonald, M.R., Cocks, P. A., and Gordon, K.C., Inorganic Chemistry, 36 (1997) 62706278.

17. Hasobe, T., Imahori, H., Fukuzumi, S., and Kamat, P.V., Journal of Material Chemistry, 13 (2003) 2515-2520.

18. Ma, R., Guo, P., Yang, L., Guo, L., Zhang, X., Nazeeruddin M.K., and Grätzel, M., Journal of Physical chemistry A, 114 (2010) 1973-1979.

19. Officer, D.L., Lodato, F., and Jolley, K.W., Inorganic Chemistry, 46 (2007) 4781-4783.

20. Nazeeruddin, M.K., Humphry-Baker, R., Officer, D.L., Campbell, W.M., Burrell, A.K., and Grätzel, Langmuir, 20 (2004 ) 6514-6517.

21. De Tacconi, N. R., Chanmanee, W., Rajeshwar, K., Rochford, J., and Galoppini, E., Journal of Physical Chemistry C, 113 (2009) 2996-3006.

22. Hasobe, T., Imahori, H, Kamat, P.V., and Fukuzumi, S., Journal of the American Chemical Society, 125 (2003) 14962-14963.

23. Imahori, H., Umeyama, T., and Ito, Seigo., Accounts of Chemical Research, 42 (2009) 1809-1818.

24. Dastoor, P.C., McNeill, C.R., Frohne, Holger, Foster, C. J., Dean, B., Fell, C.J., Belcher, W.J., Campbell, W.M., Officer, D.L., Blake, I.M., Thordarson, Pall., Crossley, M.J., Hush, N.S., and Reimers J. R., Journal of Physical Chemistry C, 111 (2007) 15415-15426.

25. Hirata, N., Kroeze, J.E., Park, T., Jones, D., Haque S.A., Holmes, A. B., and Durrant, J.R., Chemical Communications, 5 (2006) 535-537.

26. Karim, M.A., Cho, Y.-Rae., Park, J.S., Kim, S.C., Kim, H.J., Lee J. W., Gald, Y.-S., and Jin S.-H., Chemical Communications, 16 (2008) 1929-1931.

27. Campbell, W.M., Jolley, K.W., Wagner, P, Wagner, K., Walsh, P.J., Gordon, K.C., Schmidt-Mende, L., Nazeeruddin M.K., Wang, Q., Gratzel, M., and Officer, D.L., Journal of Physical chemistry C, 111 ( 2007) 11760-11762. 
28. Kitamura, T., Ikeda, M., Shigaki, K., Inoue, T., Anderson N.A., Ai, Xin., Lian, T., and Yanagida, S., Chemistry Materials, 16 (2004) 1806-1812.

29. Howie, W.H., Claeyssens, F., Miura H., and Peter, L.M., Journal of the American Chemical Society, 130 (2008) 1367-1375.

30. Wijayarathna, T. R. C. K, Aponsu, G.M. L. P, Ariyasinghe, Y.P.Y.P., Premalal, K.E.V.A, Kumara, G.K.R and Tennakone, K., Nanotechnology, 19 (2008) 485703.

31. Matsui, M., Ito, A., Kotani, M., Kubota, Y., Funabiki, K., Jin, J., Yoshida, T., Minoura, H., and Miura, H., Dyes and Pigments, 80 (2009) 233-238.

32. Kuang, D., Uchida, S., Humphry-Baker, R., Zakeeruddin, S.M., and Grätzel, M., Angewandte Chemie International Edition, 47 (2008) 1923-1927.

33. Mozer, A. J., Panda, D. K., Gambhir, S., Romeo, T., Winther-Jensen, B., and Wallace, G.G., Langmuir, 26 (2010) 1452-1455. 


\section{CONCLUSIONS}




\section{CONCLUSIONS}

The whole work presented in this thesis has aimed to investigate the optimisation conditions for in situ photo-electrochemical polymerization to prepare photo-anode for fabrication of efficient solid-state dye sensitized solar cells (ssDSCs).This was achieved by using nano-crystalline $\mathrm{TiO}_{2}$ as an electron acceptor, ruthenium dye Z907 (cis-Bis (isothiocyanato) (2, 2'-bipyridyl-4, 4'-dicarboxylato) (4, 4'-di-nonyl-2'bipyridyl) ruthenium (II), PEDOT as hole transporting material (HTM), and both metallic and non-metallic counter electrodes.

In Chapter 3, the state-of-the-art fabrication of PEDOT based solid-state dyesensitized solar cells have been successfully achieved using in situ photoelectrochemically polymerized PEDOT as hole transporting material (HTM). The device power conversion efficiency of $3 \%$ is better than previously published results. The stability and reproducibility of the solid-state dye-sensitized solar cells (ssDSCs) is improved.

The conditions of 30 minutes of PEDOT growth and $22 \mathrm{~mW} / \mathrm{cm}^{2}$ light intensity during photo-electrochemical polymerization leads to optimum performance of the devices. Other optimisation studies showed that the best thickness of $\mathrm{TiO}_{2}$ in the photo-anode is $5.5 \mu \mathrm{m}$, the best dopant anion is $\mathrm{ClO}_{4}{ }^{-}$, and the best solvent for PEP deposition of PEDOT is acetonitrile. The total polymerisation charge developed in photo-anode after photo-electrochemical polymerization by this above optimised conditions is $14 \mathrm{mC} / \mathrm{cm}^{2}$.

Grätzel cells with the same thickness of $\mathrm{TiO}_{2}$ with standard $\mathrm{I}^{-} / \mathrm{I}_{3}{ }^{-}$redox mediator were constructed and found to give a $25 \%$ higher value power conversion efficiency $(\eta \%)$ compared to the above solid-state DSCs. This was attributed to a $10 \%$ decrease in electron injection from the dye to $\mathrm{TiO}_{2}$ in the ssDSC. With higher amounts of deposited PEDOT, the $J_{\text {sc }}$ values are found to be limited by the slow dye regeneration kinetics by PEDOT. 
For the first time, a new oligomer "tris-EDOT" has been used as a HTM precursor (instead of bis-EDOT) for effective photo-electrochemical polymerization of PEDOT. The low power conversion efficiency found with this new HTM of $1.49 \%$ may be due to factors such as low transparency of PEDOT, and lower conductivities of PEDOT grown from tris-EDOT. Preliminary results with tris-EDOT indicate that further optimisation of the conditions for preparing photo-anode films with trisEDOT will pave the way to fabrication of high efficiency ssDSCs.

In Chapter 4 significant insights have been obtained into the factors causing selflimiting growth of PEDOT into dyed $\mathrm{TiO}_{2}$ substrates. The morphology of the photoanode films has been established by SEM, SEM-EDXA and AFM, and correlated with the PV performances that were limited by the limitation of growth of PEDOT into the nano-matrix voids of the dyed $\mathrm{TiO}_{2}$. It is found that a large number of pores are still present in the nano-matrix dyed $\mathrm{TiO}_{2}$. This is attributed to only partial pore filling of the $\mathrm{TiO}_{2}$ nano-matrix by PEDOT, a conclusion confirmed by crosssectional and surface morphology SEM as well as the surface topography image obtained by AFM. Further optimisation of the photo-electrochemical polymerization (PEP) of PEDOT by employing alternative dyes, HTM, or other conditions may fill all the pores by the HTM.

UV-Vis-NIR and Raman spectroscopy studies indicate the PEDOT was moderately conductive in the photo-anode. The bis-EDOT repeating unit in the photo-anode is determined for the first time by solventless MALDI (ToF) Mass Spectroscopy technique and found to be up to 19 units when PEDOT was deposited in discussed optimized conditions. The relatively slow kinetics (microsecond time scale) of regeneration of the dye cation, determined by transient absorption spectroscopy limits the solid-state DSCs performance.

In Chapter 5 attempts were made to explore the possibility of using various conducting polymer-based counter electrodes (prepared on various substrates by PEP or potentiostatic electrochemical polymerization conditions). Metal counter electrodes produced by sputtering or a metal evaporator technique were studied. Conventional $\mathrm{Au} / \mathrm{Pt}$ on ITO substrates were used as counter electrodes for ssDSCs 
but these metallic counter electrodes were found to lack reproducibility in our lab. This was attributed to the surface roughness not being well matched with the surface roughness of the photo-anode surface. A novel compressible Goretex ${ }^{\circledR}-A u-P E D O T$ counter electrode has also been employed as counter electrode and found to be a potentially useful counter electrode in ssDSC. A polymer-based counter electrode such as Goretex ${ }^{\circledR}-A u-P E D O T$, and PEDOT or polypyrrole on FTO substrate have also been found to be good as far as reproducibility is concerned, and power conversion efficiencies of up to $3 \%$ were achieved.

It has also been demonstrated that both chemically converted graphene and functionalised chemically converted graphene can be used as efficient counterelectrodes within solid-state DSCs. With the graphene electrode, an overall energy conversion efficiency $2.22 \%$ is achieved for the CCG-Cl-PEDOT/FTO counterelectrode. Interestingly, a higher $V_{\mathrm{oc}}$ is achieved with a graphene counter electrode, a higher $J_{\mathrm{sc}}$ with a Goretex®-Au-PEDOT or Au /ITO counter electrode, and a higher FF with a PEDOT/FTO based counter electrode. The all-polymer based counterelectrode and graphene based counter electrode provide cheap counter electrodes alternatives to conventional counter electrodes for ssDSCs. Moreover, the novel compressible and flexible Goretex ${ }^{\circledR}$-Au-PEDOT counter electrode will create an arena for flexible wearable, textile-based photovoltaics because it can be integrated into clothes and fabrics. Further research in this area will be beneficial for the exploitation of flexible solar cells in large industrial mass production.

Chapter 6 described the use of other low cost alternative non-ruthenium based dyes have been found to have useful properties and were used as potential photo-sensitizer for photo-electrochemical polymerization. PEDOT based ssDSCs can be fabricated utilising a zinc-porphyrin-based dye (GD2) and found to have comparable performance to ruthenium-based (Z907 dye), with relatively high open circuit voltages. These GD2 dye based devices show significant potential as a cost-effective alternative to current Ru-based dye Z907 photo-sensitizer for solid-state DSCs. The power energy conversion efficiency of the devices was as high as $2 \%$. 
Related studies were carried out using the small organic dye P-257, and D-149 dye. These dyes desorb from the photo-anode during PEP of PEDOT. The power energy conversion efficiencies of these devices were limited to $1.57 \%$ and $1 \%$, respectively. Liquid DSCs fabricated using the same $5.5 \mu \mathrm{m} \mathrm{TiO} 2$ film and P-257 dye as photosensitizer is found to have $2.7 \%$ efficiency, somewhat higher than the $\operatorname{ssDSC}(1.57 \%$ efficiency). The P-257 dye therefore has useful potential as a photo-sensitizer for both kinds of devices. Their efficiencies could be improved by further optimisation of several conditions during preparation of the working electrode.

The D-149 dye also shows comparable device performance (1\% efficiency) to the zinc-porphyrin dimer (P-10) dye. The photo-current, total polymerization and photovoltaic parameters using D-149 dye are also similar to those with P-10 dye.

Further investigations are required to identify superior low cost dyes that have higher molar extinction coefficients, broad UV-Vis absorption range, chemical and thermal stability in order to optimise photo-electrochemical polymerization for solid-state DSC.

In summary, the fabrication of $3 \%$ efficient solid state DSCs by a photoelectrochemical polymerization technique has been demonstrated. The same technique has been used to prepare different kinds of solar cells utilising various dyes, counter electrodes and hole transporting materials. 